\title{
Atratores de trajetórias para algumas classes de equações diferenciais parciais
}

\author{
Ricardo de Sá Teles
}

TESE APRESENTADA

AO INSTITUTO DE MATEMÁTICA E ESTATÍSTICA

DA

UNIVERSIDADE DE SÃO PAULO

PARA

OBTENÇÃO DO TÍTULO

$\mathrm{DE}$

DOUTOR EM CIÊNCIAS

\author{
Programa: Matemática Aplicada \\ Orientador: Prof. Dr. Luiz Augusto Fernandes de Oliveira
}

Durante o desenvolvimento deste trabalho o autor recebeu auxílio financeiro da CAPES

São Paulo, setembro de 2012 



\title{
Atratores de trajetórias para algumas classes de equações diferenciais parciais
}

\begin{abstract}
Esta versão da tese contém as correções e alterações sugeridas pela Comissão Julgadora durante a defesa da versão original do trabalho, realizada em 01/08/2012. Uma cópia da versão original está disponível no Instituto de Matemática e Estatística da Universidade de São Paulo.
\end{abstract}

Comissão Julgadora:

- Prof. Dr. Luiz Augusto Fernandes de Oliveira (orientador) - IME-USP

- Prof. Dr. Antônio Luiz Pereira - IME-USP

- Prof. Dr. Ma To Fu - ICMC-USP

- Profa. Dra. Cláudia Buttarello Gentile - UFSCar

- Prof. Dr. Arnaldo Simal do Nascimento - UFSCar 



\section{Agradecimentos}

A Deus pela presença constante em minha vida e por me dar força e esperança sempre.

Ao Prof. Dr. Luiz Augusto F. de Oliveira por ter me orientado, pela sugestão do problema e pela paciência ao longo de todo o trabalho.

Aos colegas de curso, funcionários e docentes do IME-USP, em especial aos pesquisadores do grupo de Dinâmica de Equações de Evolução.

Ao prof. Dr. Antonio de Pádua Franco Filho pelas discussões sobre Topologia.

Aos professores do exame de qualificação, Dr. Antônio Luiz Pereira e Dr. Orlando Francisco Lopes, pelas sugestões e contribuições a este trabalho.

À banca examinadora composta pelos professores Dr. Antônio Luiz Pereira, Dr. Ma To Fu, Dra. Cláudia Buttarello Gentile e Dr. Arnaldo Simal do Nascimento, pelas sugestões, correções e contribuições a este trabalho. Sou especialmente grato à profa. Dra. Cláudia Gentile pela correção cuidadosa.

Ao Ânderson da Silva Vieira pelos inúmeros auxílios no LaTex.

Aos meus pais (Nivaldo e Cidalia) e irmãos (Nilza, Neusa e Renato) pelo apoio incondicional e pela família que formamos. Não tenho palavras para descrever o papel fundamental que minha mãe (Cidalia) e meu irmão (Renato) tiveram nos momentos mais difíceis. Obrigado pelas longas conversas motivadoras, pelo incentivo e por me fazerem acreditar mais em mim.

À CAPES pela bolsa de estudo concedida. 


\section{Resumo}

Neste trabalho estudamos um problema parabólico e um problema hiperbólico que não admitem unicidade de solução. Após garantir a existência de solução para cada um desses problemas, analisamos o comportamento assintótico de suas soluções por meio da teoria do atrator de trajetórias. Nossos resultados principais demonstram, sob hipóteses apropriadas, a semicontinuidade superior das famílias de atratores de trajetórias quando o coeficiente de difusão é grande.

Palavras-chave: atrator de trajetórias, semicontinuidade superior, difusão grande, não unicidade de solução, Faedo-Galerkin 


\section{Abstract}

In this work we study a parabolic problem and a hyperbolic problem that not admit uniqueness of solution. After to ensure existence of solution for each of these problems, we analyze the asymptotic behavior of their solutions by means of the theory of trajectory attractors. Our main results demonstrate, under appropriate assumptions, the upper semicontinuity of families of trajectory attractors when the diffusion coefficient is large.

Keywords: trajectory attractors, upper semicontinuity, large diffusion, non-uniqueness of solution, Faedo-Galerkin 


\section{Sumário}

$\begin{array}{ll}\text { Introdução } & 1\end{array}$

$\begin{array}{llr}1 & \text { Resultados preliminares } & 7\end{array}$

1.1 Espaços de funções, teoremas de imersão e resultados de Análise . . . . . . . . 7

1.1.1 Os espaços $H^{s}(\Omega), H^{s}(\Gamma)$ e o traço da derivada normal . . . . . . . . . 18

1.2 Coletânea de definições e resultados de Topologia . . . . . . . . . . . . . . . . 24

1.3 Semigrupos em espaços topológicos e atratores . . . . . . . . . . . . . . . 25

1.4 Aplicações para $(\mathcal{M}, \mathcal{T})$ - atratores . . . . . . . . . . . . . . . . . . . 28

2 Construção do atrator de trajetórias de equações de evolução abstratas 29

2.1 Equações diferenciais ordinárias autônomas . . . . . . . . . . . . . . . . . . 29

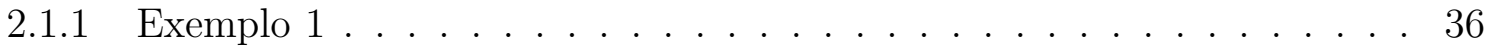

2.1.2 Exemplo 2: sistemas de primeira ordem . . . . . . . . . . . . 38

2.1.3 Exemplo 3: sistemas de segunda ordem. . . . . . . . . . . . . . . . 39

2.1.4 Dependência de um parâmetro . . . . . . . . . . . . . . . . 42

2.2 Equações de evolução abstratas . . . . . . . . . . . . . . . . . . . . 47

2.2.1 Atrator global fraco . . . . . . . . . . . . . . . 52 
3 Atrator de trajetórias de uma equação parabólica 55

3.1 Construção do atrator de trajetórias de um problema parabólico . . . . . . . . 58

3.2 Semicontinuidade superior de uma família de atratores de trajetórias . . . . . 75

3.3 Difusão grande . . . . . . . . . . . . . . . . . . . . . . . . . . 81

4 Atrator de trajetórias de uma equação hiperbólica 89

4.1 Construção do atrator de trajetórias de um problema hiperbólico . . . . . . . . 91

4.1.1 Atratores globais para equações hiperbólicas . . . . . . . . . . . . 107

4.2 Semicontinuidade superior de uma família de atratores de trajetórias . . . . 109

4.2.1 Primeiro problema . . . . . . . . . . . . . . . . . 109

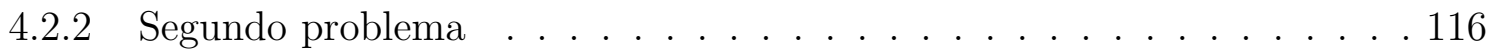

4.3 Difusão grande . . . . . . . . . . . . . . . . . . . . . 123

\section{Bibliografia}




\section{Introdução}

Um problema importante no estudo das equações de evolução é a investigação do comportamento assintótico de suas soluções. Muitos livros e artigos tratam desse tema no caso em que o problema de Cauchy correspondente tem solução única, o que determina, em geral, a boa definição e a continuidade do operador solução associado. Esse operador é definido, normalmente, sobre um espaço métrico completo ou espaço de Banach, denominado espaço de fase, e constitui um semigrupo. Se esse semigrupo é dissipativo, então é possível obter um atrator global que, em suma, é um conjunto que atrai todas as trajetórias do semigrupo quando o tempo tende para o infinito. As referências principais sobre esse assunto são Babin A. V. and Vishik, M. I. [2], Hale, J. [23] e Temam, R. [46].

O método clássico para a construção do atrator global baseia-se, fundamentalmente, na propriedade de unicidade de solução do problema de Cauchy. Porém, há uma classe significativa de problemas mal postos cuja propriedade de unicidade não ocorre ou encontra-se em aberto. Nesse caso, também há interesse no estudo do comportamento assintótico das soluções, mas a estratégia utilizada nos problemas bem postos não se aplica diretamente e, portanto, outros métodos devem ser buscados; dentre eles, destacamos a teoria do atrator de trajetórias desenvolvida por V. V. Chepyzhov e M. I. Vishik. Uma exposição sobre esse assunto pode ser encontrada nas referências [9] e [10] dos mesmos autores . Indicamos também a referência Kapustyan, O. V. and Valero, J. [27] para uma exposição a respeito de outras abordagens existentes na literatura para tratar de problemas sem unicidade e possíveis relações entre elas.

O nosso interesse nesse trabalho é estudar o atrator de trajetórias para investigar o comportamento assintótico das soluções de equações diferenciais parciais autônomas que não gozam da propriedade de unicidade de solução. O roteiro para a construção do atrator será apresentado no Capítulo 2. Em Miranville, A. and Zelik, S. [37], os autores observam que essa abordagem evita as "não amigáveis" aplicações multívocas e permite o estudo de problemas mal postos usando a teoria clássica de atratores para semigrupos unívocos, uma vez que o atrator de trajetórias é o atrator global para um semigrupo translação definido sobre o conjunto das soluções do problema sob consideração. Este conjunto está contido no conjunto de funções $\mathcal{F}_{l o c}^{+}$sobre o qual definimos uma topologia apropriada. O conjunto de soluções 
dotado da topologia induzida será denominado espaço de trajetórias e será naturalmente invariante sob a ação do semigrupo. Vamos ainda definir um subespaço de $\mathcal{F}_{l o c}^{+}$munido de uma norma que o torna um espaço de Banach e que nos permitirá considerar conjuntos limitados. O atrator de trajetórias atrairá todo subconjunto limitado contido na interseção do espaço de trajetórias com um conjunto absorvente a ser determinado.

Observamos que é também possível estudar o atrator de trajetórias para problemas cuja unicidade de solução já foi provada. Nesse caso, o atrator de trajetórias $\mathfrak{A}$ consiste de todas as trajetórias $u$ tais que os valores $u(t), t \geqslant 0$, pertencem ao atrator global usual $\mathcal{A}$, ou seja, o atrator global é uma seção do atrator de trajetórias.

Na literatura há resultados conhecidos para equações de reação e difusão da forma

$$
\frac{\partial u}{\partial t}=D \Delta u-f(u)
$$

e equações hiperbólicas da forma

$$
\frac{\partial^{2} u}{\partial t^{2}}+b \frac{\partial u}{\partial t}=D \Delta u-f(u)
$$

com condições de fronteira de Neumann, $u=\left(u_{1}, \ldots, u_{m}\right)^{t}$ e $D=\operatorname{diag}\left(d_{1}, \ldots, d_{m}\right)$, que demonstram que esses problemas tem comportamento assintótico descrito por um sistema de equações diferenciais ordinárias quando os coeficientes de difusão tendem para o infinito. Não encontramos referências para esse estudo no contexto de problemas sem unicidade de solução e envolvendo o atrator de trajetórias. Isso serviu como grande motivação para a realização desse trabalho e é nesse sentido que daremos a nossa contribuição. Os nossos resultados principais encontram-se nas Seções 3.3 e 4.3. As demonstrações baseiam-se em resultados de Análise Funcional, teoria das distribuições vetoriais e topologia.

Este trabalho está organizado da seguinte forma: no Capítulo 1, reunimos as definições e os resultados necessários para a compreensão dos demais capítulos. No Capítulo 2, como já dissemos, procuramos axiomatizar a construção do atrator de trajetórias para as equações de evolução abstratas. No Capítulo 3, Seção 3.1, estudamos o problema parabólico

$$
\left\{\begin{array}{l}
\frac{\partial u}{\partial t}=d \Delta u-f(u)+|u|^{\alpha-1} u \quad(t, x) \in(0, \infty) \times \Omega \\
\frac{\partial u}{\partial \nu}=0 \quad(t, x) \in(0, \infty) \times \Gamma
\end{array}\right.
$$

onde $\Omega \subset \mathbb{R}^{n}(n \geqslant 3)$ é um subconjunto aberto, limitado, conexo, com fronteira suave $\Gamma=\partial \Omega$ e para cada ponto $x \in \Gamma, \nu=\nu(x)$ indica o vetor normal exterior unitário a $\Gamma$ no ponto $x$. Além disso, $d>0$ está fixado, $\alpha \in(0,1)$ e $f: \mathbb{R} \rightarrow \mathbb{R}$ é uma função de classe $C^{1}$ satisfazendo as seguintes hipóteses: existem constantes positivas $c_{1}, c_{2}, c_{3}$ e $2<p<\frac{2 n}{n-2}$ tais que

$$
f^{\prime}(u) \geqslant c_{f}
$$




$$
c_{1}|u|^{p}-c_{3} \leqslant f(u) \cdot u
$$

$\mathrm{e}$

$$
|f(u)|^{\frac{p}{p-1}} \leqslant c_{2}\left(|u|^{p}+1\right),
$$

para todo $u \in \mathbb{R}$. Destacamos que a desigualdade (0.3) é denominada condição de dissipatividade de $f$. Depois de definir os espaços em que trabalharemos e o conceito de solução, para $u_{0} \in L^{2}(\Omega)$ demonstramos, via método de Faedo-Galerkin, a existência de pelo menos uma solução fraca global. Vamos considerar as soluções obtidas tomando-se o dado inicial $u_{0} \in H_{d}^{1}(\Omega)$ e daremos uma descrição do comportamento assintótico das mesmas a partir da construção do atrator de trajetórias. Na Seção 3.2, obteremos a semicontinuidade superior da família de atratores de trajetórias $\left\{\mathfrak{A}_{\epsilon}: \epsilon \in\left[0, \epsilon_{0}\right]\right\}$ em $\epsilon=0$ para o seguinte problema envolvendo um parâmetro $\epsilon$ pequeno

$$
\left\{\begin{array}{l}
\frac{\partial u}{\partial t}=d \Delta u-f(u)+\epsilon|u|^{\alpha-1} u \quad(t, x) \in(0, \infty) \times \Omega \\
\frac{\partial u}{\partial \nu}=0 \quad(t, x) \in(0, \infty) \times \Gamma,
\end{array}\right.
$$

onde $f$ satisfaz as hipóteses (0.2)-(0.4). Para esse problema, demonstramos o seguinte resultado: $\mathfrak{A}_{\epsilon} \subset \mathcal{O}\left(\mathfrak{A}_{0}\right)$, quando $\epsilon \rightarrow 0$, onde $\mathcal{O}$ indica uma vizinhança (numa topologia adequada) do atrator de trajetórias do problema limite $\operatorname{com} \epsilon=0$. Na Seção 3.3, consideramos o caso em que o coeficiente de difusão é grande no problema a seguir

$$
\left\{\begin{array}{l}
\frac{\partial u}{\partial t}=d \Delta u-f(u)+|u|^{\alpha-1} u \quad(t, x) \in(0, \infty) \times \Omega \\
\frac{\partial u}{\partial \nu}=0 \quad(t, x) \in(0, \infty) \times \Gamma
\end{array}\right.
$$

onde $\Omega \subset \mathbb{R}^{n}(n \geqslant 3)$ é um subconjunto aberto, limitado, conexo, com fronteira suave $\Gamma \mathrm{e}$ para cada ponto $x \in \Gamma, \nu=\nu(x)$ indica o vetor normal exterior unitário a $\Gamma$ no ponto $x$. Além disso, $d>0, \alpha \in(0,1)$ e $f: \mathbb{R} \rightarrow \mathbb{R}$ é uma função de classe $C^{1}$ satisfazendo as seguintes hipóteses: existem constantes positivas $c_{1}, c_{2}, c_{3}, C, c_{f}$ e $2<p<\frac{2 n}{n-2}$ tais que

$$
\begin{gathered}
f^{\prime}(u) \geqslant c_{f}, \\
\left|f\left(u_{1}\right)-f\left(u_{2}\right)\right| \leqslant C|u-v|\left(1+\left|u_{1}\right|^{p-2}+\left|u_{2}\right|^{p-2}\right), \\
c_{1}|u|^{p}-c_{3} \leqslant f(u) \cdot u
\end{gathered}
$$

e

$$
|f(u)|^{\frac{p}{p-1}} \leqslant c_{2}\left(|u|^{p}+1\right),
$$


Introdução

para quaisquer $u, u_{1}, u_{2} \in \mathbb{R}$. Mais especificamente, demonstramos que a família de atratores de trajetórias $\left\{\mathfrak{A}_{d}\right\}_{d}$ é semicontínua superiormente em $d=+\infty$, onde $\mathfrak{A}_{\infty}$ é o atrator de trajetórias da equação diferencial ordinária

$$
\frac{d u}{d t}=-f(u)+|u|^{\alpha-1} u
$$

No Capítulo 4, Seção 4.1, estudamos o problema hiperbólico

$$
\left\{\begin{array}{l}
\frac{\partial^{2} u}{\partial t^{2}}+2 \gamma \frac{\partial u}{\partial t}=d \Delta u-f(u)+|u|^{\alpha-1} u \quad(t, x) \in(0, \infty) \times \Omega \\
\frac{\partial u}{\partial \nu}=0 \quad(t, x) \in(0, \infty) \times \Gamma,
\end{array}\right.
$$

onde $\Omega \subset \mathbb{R}^{n}(n \geqslant 3)$ é um subconjunto aberto, limitado, conexo, com fronteira suave $\Gamma \mathrm{e}$ para cada ponto $x \in \Gamma, \nu=\nu(x)$ indica o vetor normal exterior unitário a $\Gamma$ no ponto $x$. Além disso, $\gamma>0, d>0$ está fixado, $\alpha \in(0,1)$ e $f: \mathbb{R} \rightarrow \mathbb{R}$ é uma função de classe $C^{1}$ satisfazendo as seguintes hipóteses: existem constantes positivas $\gamma_{0}, \gamma_{1}, \gamma_{2}, c_{1}, c_{2}$ e $2<p<\frac{2 n}{n-2}$ tais que

$$
\begin{gathered}
|f(u)| \leqslant \gamma_{0}\left(|u|^{p-1}+1\right), \\
F(u)=\int_{0}^{u} f(w) d w, \quad F(u) \geqslant \gamma_{1}|u|^{p}-c_{1}, \\
f(u) \cdot u \geqslant \gamma_{2} F(u)-c_{2},
\end{gathered}
$$

para todo $u \in \mathbb{R}$. Depois de definir os espaços em que trabalharemos e o conceito de solução, para $u_{0} \in H^{1}(\Omega) \cap L^{p}(\Omega)$ demonstramos, via método de Faedo-Galerkin, a existência de pelo menos uma solução fraca global e daremos a descrição do comportamento assintótico das soluções a partir da construção do atrator de trajetórias. Na Seção 4.2, obteremos a semicontinuidade superior da família de atratores de trajetórias $\left\{\mathfrak{A}_{\epsilon}: \epsilon \in\left[0, \epsilon_{0}\right]\right\}$ em $\epsilon=0$ para os seguintes problemas envolvendo um parâmetro $\epsilon$ pequeno

$$
\left\{\begin{array}{l}
\frac{\partial^{2} u}{\partial t^{2}}+2 \gamma \frac{\partial u}{\partial t}=d \Delta u-f(u)+\epsilon|u|^{\alpha-1} u \quad(t, x) \in(0, \infty) \times \Omega \\
\frac{\partial u}{\partial \nu}=0 \quad(t, x) \in(0, \infty) \times \Gamma
\end{array}\right.
$$

$\mathrm{e}$

$$
\left\{\begin{array}{l}
\epsilon \frac{\partial^{2} u}{\partial t^{2}}+2 \gamma \frac{\partial u}{\partial t}=a \Delta u-f(u)+|u|^{\alpha-1} u \quad(t, x) \in(0, \infty) \times \Omega \\
\frac{\partial u}{\partial \nu}=0 \quad(t, x) \in(0, \infty) \times \Gamma .
\end{array}\right.
$$

Para esses problemas, demonstramos o seguinte resultado: $\mathfrak{A}_{\epsilon} \subset \mathcal{O}\left(\mathfrak{A}_{0}\right)$, quando $\epsilon \rightarrow 0$, onde $\mathcal{O}$ indica uma vizinhança (numa topologia conveniente) do atrator de trajetórias do problema 
limite $\operatorname{com} \epsilon=0$. Na Seção 4.3, consideramos o caso em que o coeficiente de difusão $d \rightarrow+\infty$ no problema a seguir

$$
\left\{\begin{array}{l}
\frac{\partial^{2} u}{\partial t^{2}}+2 \gamma \frac{\partial u}{\partial t}=a \Delta u-f(u)+|u|^{\alpha-1} u \quad(t, x) \in(0, \infty) \times \Omega \\
\frac{\partial u}{\partial \nu}=0 \quad(t, x) \in(0, \infty) \times \Gamma
\end{array}\right.
$$

onde $\Omega \subset \mathbb{R}^{n}(n \geqslant 3)$ é um subconjunto aberto, limitado, conexo, com fronteira suave $\Gamma \mathrm{e}$ para cada ponto $x \in \Gamma, \nu=\nu(x)$ indica o vetor normal exterior unitário a $\Gamma$ no ponto $x$. Além disso, $\gamma>0, d>0, \alpha \in(0,1)$ e $f: \mathbb{R} \rightarrow \mathbb{R}$ é uma função de classe $C^{1}$ satisfazendo as seguintes hipóteses: existem constantes positivas $C_{f}, \gamma_{0}, \gamma_{1}, \gamma_{2}, c_{1}, c_{2}$ e $2<p<\frac{2 n}{n-2}$ tais que

$$
\begin{gathered}
\left|f\left(u_{1}\right)-f\left(u_{2}\right)\right| \leqslant C\left|u_{1}-u_{2}\right|\left(1+\left|u_{1}\right|^{p-2}+\left|u_{2}\right|^{p-2}\right), \\
|f(u)| \leqslant \gamma_{0}\left(|u|^{p-1}+1\right), \\
F(u)=\int_{0}^{u} f(w) d w, \quad F(u) \geqslant \gamma_{1}|u|^{p}-c_{1}, \\
f(u) \cdot u \geqslant \gamma_{2} F(u)-c_{2},
\end{gathered}
$$

para quaisquer $u, u_{1}, u_{2} \in \mathbb{R}$. Vamos demonstrar que a família de atratores de trajetórias $\left\{\mathfrak{A}_{d}\right\}_{d}$ é semicontínua superiormente em $d=+\infty$, onde $\mathfrak{A}_{\infty}$ é o atrator da equação diferencial ordinária

$$
\frac{d^{2} u}{d t^{2}}+2 \gamma \frac{d u}{d t}=-f(u)+|u|^{\alpha-1} u
$$

Observamos que os problemas estudados nesse trabalho não tem unicidade de solução devido a presença da função $\sigma(u)=|u|^{\alpha-1} u(\alpha \in(0,1))$ que não é Lipschitz, mas é uma função holderiana de ordem $\alpha$. O problema (0.1) com condição de Dirichlet foi considerado por Yue, G.; Zhong, C. [51], onde os autores demonstraram o resultado: $\mathfrak{A}^{\prime} \subset \mathcal{O}\left(\mathfrak{A}_{0}^{\prime}\right)$, onde $\mathcal{O}$ indica uma vizinhança do atrator de trajetórias de (0.1) e $\mathfrak{A}_{\epsilon}^{\prime}$ é o atrator global da equação com uma perturbação no termo de reação

$$
\frac{\partial u}{\partial t}=d \Delta u-f(u)+(|u|+\epsilon)^{\alpha-1} u .
$$




\section{Resultados preliminares}

O objetivo deste capítulo é reunir alguns resultados que serão utilizados ao longo do trabalho. Na Seção 1.1 identificamos os espaços que aparecerão com frequência no texto. A Seção 1.2 é reservada para relembrar algumas definições e resultados de topologia. Nas Seções 1.3 e 1.4 trataremos dos semigrupos definidos sobre um espaço topológico e atratores. A maioria dos resultados foram retirados do livro de Chepyzhov, V. V. and Vishik, M. I. [9] e as demonstrações serão em grande parte omitidas, porém indicaremos as referências adequadas.

\subsection{Espaços de funções, teoremas de imersão e resulta- dos de Análise}

Em todo este trabalho, $\Omega \subset \mathbb{R}^{n}$ denotará um subconjunto aberto, limitado, conexo e com fronteira suave. Denotaremos por $\partial_{i}$ o operador de derivada parcial com respeito a $x_{i}$, isto é, $\partial_{i} u=\frac{\partial u}{\partial x_{i}}(i=1, \ldots, n)$, e por $\partial_{t}$ a derivada parcial com respeito a $t$, isto é, $\partial_{t} u=\frac{\partial u}{\partial t}$. Usaremos também as notações " $u$ " e $u_{t}$ para a derivada de $u$ com relação a $t$.

O espaço das funções reais contínuas limitadas em $\Omega$ será denotado por $C(\Omega)$ e a norma nesse espaço é a usual, ou seja, $\|u\|_{C(\Omega)}=\sup _{x \in \Omega}|u(x)|$.

Os espaços $C^{k}(\Omega), k \in \mathbb{Z}_{+}$, consistem das funções reais com derivadas parciais até ordem $k$ contínuas e limitadas em $\Omega$ e a norma nesse espaço será definida por $\|u\|_{C^{k}(\Omega)}=\sup \left\{\left\|\partial^{\alpha} u\right\|_{C(\Omega)}: \alpha \in \mathbb{Z}_{+}^{n},|\alpha| \leqslant k\right\}$. Aqui $\alpha$ é um multiíndice definido da forma padrão.

Seja $1 \leqslant p<\infty$. Denotaremos por $L^{p}(\Omega)$ o espaço das funções mensuráveis $f: \Omega \rightarrow$ $\mathbb{R}$ tais que $|f|^{p}$ é Lebesgue-integrável, munido da norma $\|u\|_{0, p}=\|u\|_{L^{p}}=\left(\int_{\Omega}|u(x)|^{p} d x\right)^{\frac{1}{p}}$. $L^{\infty}(\Omega)$ indicará o espaço das funções limitadas quase sempre com norma $\|u\|_{0, \infty}=\|u\|_{L^{\infty}}=$ $\operatorname{ess} \sup \{|u(x)|: x \in \Omega\}$.

A norma no espaço de Sobolev $W^{l, p}(\Omega), l \in \mathbb{Z}_{+}, 1 \leqslant p<+\infty$, será definida pela 
1 Resultados preliminares

fórmula

$$
\|u\|_{l, p}=\left(\sum_{|\alpha| \leqslant l}\left\|\partial^{\alpha} u\right\|_{L^{p}}^{p}\right)^{\frac{1}{p}} .
$$

Se $p=2$, o espaço de Sobolev $W^{l, 2}(\Omega)$ é um espaço de Hilbert denotado por $H^{l}(\Omega)$ e o produto escalar em $H^{l}(\Omega)$ será definido por $\langle u, v\rangle_{l}=\sum_{|\alpha| \leqslant l} \int_{\Omega} \partial^{\alpha} u(x) \partial^{\alpha} v(x) d x$. O espaço $W^{l, p}(\Omega)$ é o fecho de $C^{l}(\Omega)$ na norma (1.1).

Indicaremos por $H^{-l}(\Omega), l>0$, o espaço dual de $H^{l}(\Omega)$ e a norma nesse espaço será definida por $\|u\|_{-l}=\sup \left\{\frac{|\langle v, u\rangle|}{\|v\|_{l}}: v \in H^{l}(\Omega), v \neq 0\right\}$.

Sejam $F_{1}$ e $F_{2}$ espaços de Banach e suponha que $F_{2}$ é um subespaço vetorial de $F_{1}$. Dizemos que $F_{2}$ está imerso em $F_{1}$ se o operador inclusão for contínuo com respeito as topologias geradas pelas normas de $F_{1}$ e $F_{2}$ e denotaremos esse fato por $F_{2} \hookrightarrow F_{1}$. Observamos que a imersão $F_{2} \hookrightarrow F_{1}$ implica que $\|u\|_{F_{1}} \leqslant C\|u\|_{F_{2}}$, onde $C$ é uma constante independente de $u \in F_{2}$. Além disso, se o operador imersão é compacto, então diremos que $F_{2}$ está imerso compactamente em $F_{1}$ e denotaremos $F_{2} \stackrel{\text { c }}{\hookrightarrow} F_{1}$.

Vamos enunciar alguns resultados de imersão, cujas demonstrações podem ser encontradas em Triebel, H. [49], Teorema 4.6.2, página 328.

Teorema 1.1.1. Sejam $\Omega \subset \mathbb{R}^{n}$ um subconjunto aberto, conexo e limitado, $l_{1}, l_{2} \in \mathbb{Z}_{+} e$ $1<p_{1}, p_{2}<+\infty$.

1. Se $l_{2} \geqslant l_{1}, p_{2} \geqslant p_{1}$ e $\frac{l_{2}}{n}-\frac{1}{p_{2}} \geqslant \frac{l_{1}}{n}-\frac{1}{p_{1}}$, então $W^{l_{2}, p_{2}}(\Omega) \hookrightarrow W^{l_{1}, p_{1}}(\Omega)$.

2. Se $l_{2}>l_{1}, p_{2} \geqslant p_{1}$ e $\frac{l_{2}}{n}-\frac{1}{p_{2}}>\frac{l_{1}}{n}-\frac{1}{p_{1}}$, então $W^{l_{2}, p_{2}}(\Omega) \stackrel{c}{\hookrightarrow} W^{l_{1}, p_{1}}(\Omega)$.

Teorema 1.1.2. Sejam $\Omega \subset \mathbb{R}^{n}$ um subconjunto aberto, conexo e limitado e $1<p_{1}<\infty$. Se $0 \leqslant l_{2}<l_{1}-\frac{n}{p_{1}}$, então $W^{l_{1}, p_{1}}(\Omega) \hookrightarrow C^{l_{2}}(\Omega)$.

Sejam $E$ um espaço de Banach e $E^{\prime}$ o seu dual. Se $E \hookrightarrow L^{2}(\Omega)$, então cada função $\varphi$ em $L^{2}(\Omega)$ define um funcional que atua sobre $E$ pela fórmula $\varphi(u)=\langle u, \varphi\rangle$. Logo a imersão $L^{2}(\Omega) \hookrightarrow E^{\prime}$ está definida. A proposição a seguir permite estender esse resultado de imersão para $L^{q}(\Omega) \operatorname{com} q<2$.

Proposição 1.1.3. Se $E$ é um espaço de Banach e $E \hookrightarrow H^{s}(\Omega)$ (o caso $E=H^{s}(\Omega)$ não é excluído), então $L^{q}(\Omega) \hookrightarrow E^{\prime} e\|u\|_{E^{\prime}} \leqslant C_{q}\|u\|_{0, q}$ para todo $q \geqslant 1$ satisfazendo $\frac{1}{q} \leqslant \min \left\{1, \frac{1}{2}+\frac{s}{n}\right\}$, excluindo o caso $\frac{s}{n}=\frac{1}{2}$ quando $L^{q}(\Omega) \hookrightarrow E^{\prime}$ para todo $q>1$.

Demonstração: Ver Chepyzhov, V. V. and Vishik, M. I. [9], Proposição 1.1, página 30. 
Enunciamos a seguir a Desigualdade de Gagliardo-Nirenberg da forma que encontramos em Chepyzhov, V. V. and Vishik, M. I. [9], Teorema 1.3, página 30.

Teorema 1.1.4. Sejam $1 \leqslant p_{1}, p_{2} \leqslant \infty, r, l \in \mathbb{Z}_{+}, 0 \leqslant r<l$. Se $u \in L^{p_{1}}(\Omega) \cap W^{l, p_{2}}(\Omega)$ e $\theta$ e p são números positivos tais que o número

$$
\theta=\frac{\frac{n}{p}-\frac{n}{p_{1}}-r}{\frac{n}{p_{2}}-\frac{n}{p_{1}}-l}
$$

satisfaz a desigualdade $\frac{r}{l} \leqslant \theta<1$, então existe uma constante positiva $C$ tal que

$$
\|u\|_{r, p} \leqslant C\|u\|_{0, p_{1}}^{1-\theta}\|u\|_{l, p_{2}}^{\theta}
$$

Definição 1.1.5. Dizemos que uma sequência de funções $\left\{\phi_{\nu}\right\} \subset C_{0}^{\infty}(\Omega)$ é convergente para zero quando as seguintes condições forem satisfeitas

(i) os suportes de todas as funções $\phi_{\nu}$ estão contidos num conjunto compacto fixo $K \subset \Omega$;

(ii) para cada $\alpha \in \mathbb{N}^{n}$, a sequência $\left\{\partial^{\alpha} \phi_{\nu}\right\}$ converge para zero uniformemente em $K$.

Definição 1.1.6. O espaço vetorial $C_{0}^{\infty}(\Omega)$ com a noção de convergência dada em 1.1 .5 é representado por $\mathcal{D}(\Omega)$ e denominado espaço das funções testes em $\Omega$.

Definição 1.1.7. Uma distribuição (ou distribuição escalar) sobre $\Omega$ é todo funcional linear $T: \mathcal{D}(\Omega) \rightarrow \mathbb{R}$ que é contínuo no sentido da convergência definida sobre $\mathcal{D}(\Omega)$. O conjunto de todas as distribuições sobre $\Omega$ é um espaço vetorial que indicaremos por $\mathcal{D}^{\prime}(\Omega)$. Neste espaço vetorial diremos que uma sequência $\left\{T_{\nu}\right\}$ de vetores de $\mathcal{D}^{\prime}(\Omega)$ converge para zero em $\mathcal{D}^{\prime}(\Omega)$ quando para toda função teste $\varphi \in \mathcal{D}(\Omega)$ a sequência $\left\{\left\langle\varphi, T_{\nu}\right\rangle\right\}$ converge para zero em $\mathbb{R}$.

Neste trabalho consideraremos com frequência funções $u(t, x)$, dependendo das variáveis $t \in[\tau, T] \subset \mathbb{R}$ e $x \in \Omega$, como funções de $t \in[\tau, T]$ com valores num espaço de Banach $E$, cujos elementos são funções definidas em $\Omega$. As funções $u$ podem pertencer aos espaços $C^{k}([\tau, T] ; E), L^{\infty}(\tau, T ; E)$ ou $L^{p}(\tau, T ; E)$ com $p \geqslant 1$, onde $E=W^{l, p}(\Omega)$ ou $C^{k}(\Omega)$. As respectivas normas são definidas por

$$
\begin{gathered}
\|u\|_{C^{k}([\tau, T] ; E)}=\sup \left\{\left\|\partial_{t}^{j} u(t)\right\|_{E}: t \in[\tau, T], j=0, \ldots, k\right\} \\
\|u\|_{L^{\infty}(\tau, T ; E)}=\operatorname{ess} \sup \left\{\|u(s)\|_{E}: s \in[\tau, T]\right\} \\
\|u\|_{L^{p}(\tau, T ; E)}^{p}=\int_{\tau}^{T}\|u(t)\|_{E}^{p} d t
\end{gathered}
$$

Definição 1.1.8. Uma distribuição vetorial sobre $[0, T]$ com valores em $X$ é uma função $u: \mathcal{D}(0, T) \rightarrow X$ linear e contínua. O conjunto dessas aplicações lineares será denotado por $\mathcal{D}^{\prime}(0, T ; X)$. 
1 Resultados preliminares

Observação 1.1.1. Sejam $u \in L^{p}(0, T ; X), 1 \leqslant p \leqslant \infty e \varphi \in \mathcal{D}(0, T)$. Consideremos a função $T_{u}: \mathcal{D}(0, T) \rightarrow X$ dada por

$$
\left\langle\varphi, T_{u}\right\rangle=\int_{0}^{T} \varphi(t) u(t) d t,
$$

onde a integral é calculada no sentido de Bochner em $X$. A aplicação $T_{u}$ é uma distribuição vetorial sobre $[0, T]$ com valores em $X$ e é univocamente determinada por $u \in L^{p}(0, T ; X)$. Dessa forma, podemos identificar u com a distribuição $T_{u}$ e $L^{p}(0, T ; X) \hookrightarrow \mathcal{D}^{\prime}(0, T ; X)$.

Definição 1.1.9. Suponhamos $p, p_{0} \geqslant 1$. Dados $E$ e $E_{0}$, espaços de Banach satisfazendo $E \hookrightarrow E_{0}$, sejam $u \in L^{p}(\tau, T ; E)$ e $v \in L^{p_{0}}\left(\tau, T ; E_{0}\right)$. Diremos que $v$ é a derivada de $u$ no sentido de distribuição em $\mathcal{D}^{\prime}\left((\tau, T) ; E_{0}\right)$ se

$$
\int_{\tau}^{T} v(t) \varphi(t) d t=-\int_{\tau}^{T} u(t) \partial_{t} \varphi(t) d t,
$$

para toda $\varphi \in C_{0}^{\infty}((\tau, T) ; \mathbb{R})$ e escreveremos $v=\partial_{t} u$ para indicar que $v$ é a derivada de u no sentido de distribuição em $\mathcal{D}^{\prime}\left((\tau, T) ; E_{0}\right)$. A integral é tomada em $L^{p_{0}}\left(\tau, T ; E_{0}\right)$.

Duas boas referências para o estudo dos espaços $L^{p}(0, T ; E)$ e de distribuições vetoriais são Matos, M. [32] e Medeiros, L. A. [33].

O próximo resultado é um lema técnico sobre as derivadas de funções com valores num espaço de Banach.

Lema 1.1.10. Sejam E um espaço de Banach e $E^{\prime}$ o seu dual. Se $u, v \in L^{1}(\tau, T ; E)$, então as seguintes afirmações são equivalentes

(i) v é a derivada de u no sentido de distribuição em $\mathcal{D}^{\prime}((\tau, T) ; E)$;

(ii) $u$ é quase sempre uma primitiva de $v$, isto é, existe $\xi \in E$ satisfazendo $u(t)=\xi+\int_{\tau}^{T} v(s) d s$ para quase todo $t \in(\tau, T)$, ou seja, u é uma função absolutamente contínua em $E$.

(iii) $\frac{d}{d t}\langle u(t), \eta\rangle=\langle v(t), \eta\rangle$, para todo $\eta \in E^{\prime}$, isto é, a função $t \rightarrow\langle v(t), \eta\rangle$ é a derivada de $t \rightarrow\langle u(t), \eta\rangle$ no sentido de distribuição em $\mathcal{D}^{\prime}((\tau, T) ; \mathbb{R})$, para todo $\eta \in E^{\prime} ;$

(iv) temos

$$
\int_{\tau}^{T}\langle v(t), \psi(t)\rangle d t=-\int_{\tau}^{T}\left\langle u(t), \partial_{t} \psi(t)\right\rangle d t,
$$

para todo $\psi \in C_{0}^{1}\left([\tau, T] ; E^{\prime}\right)$.

Demonstração: Ver Temam, R. [48], Lema 1.1, página 169. 
Se $p_{1}, p_{0} \geqslant 1$ e $E_{0}, E_{1}$ são espaços de Banach satisfazendo $E_{1} \hookrightarrow E_{0}$, consideramos o espaço

$$
W_{p_{1}, p_{0}}\left(0, T ; E_{1}, E_{0}\right)=\left\{\psi: \psi \in L^{p_{1}}\left(0, T ; E_{1}\right), \psi^{\prime} \in L^{p_{0}}\left(0, T ; E_{0}\right)\right\}
$$

munido da norma

$$
\|\psi\|_{W_{p_{1}, p_{0}}}=\left(\int_{0}^{T}\|\psi(s)\|_{E_{1}}^{p_{1}} d s\right)^{\frac{1}{p_{1}}}+\left(\int_{0}^{T}\left\|\psi^{\prime}(s)\right\|_{E_{0}}^{p_{0}} d s\right)^{\frac{1}{p_{0}}} .
$$

A norma no espaço $W_{\infty, p_{0}}\left(0, T ; E_{1}, E_{0}\right)$ é definida por

$$
\|\psi\|_{W_{\infty, p_{0}}}=\operatorname{ess} \sup \left\{\|\psi(s)\|_{E_{1}}: s \in[0, T]\right\}+\left(\int_{0}^{T}\left\|\psi^{\prime}(s)\right\|_{E_{0}}^{p_{0}} d s\right)^{\frac{1}{p_{0}}} .
$$

Vejamos alguns resultados de compacidade devido a Aubin-Lions.

Teorema 1.1.11. Sejam $1<p_{0}, p_{1}<\infty, T>0$ e $E_{1}, E, E_{0}$ espaços de Banach. Se $E_{1} \stackrel{c}{\hookrightarrow} E \hookrightarrow E_{0}$, ent $\tilde{a} o W_{p_{1}, p_{0}}\left(0, T ; E_{1}, E_{0}\right) \stackrel{c}{\hookrightarrow} L^{p_{1}}(0, T ; E)$.

Demonstração: Ver Chepyzhov, V. V. and Vishik, M. I. [9] (Apêndice A, página 345) ou Lions, J. L. [29] (Capítulo 1, Teorema 5.1, página 58) ou Temam, R. [48] (Capítulo 3, Teorema 2.1, página 184).

Em decorrência do Teorema de Compacidade de Aubin-Lions segue que se $\left\{u_{m}\right\}_{m \in \mathbb{N}}$ é uma sequência limitada em $L^{p_{1}}\left(0, T ; E_{1}\right)$ e $\left\{u_{m}^{\prime}\right\}_{m \in \mathbb{N}}$ é uma sequência limitada em $L^{p_{0}}\left(0, T ; E_{0}\right)$, então $\left\{u_{m}\right\}_{m \in \mathbb{N}}$ é limitada em $W_{p_{1}, p_{0}}\left(0, T ; E_{1}, E_{0}\right)$. Assim, da imersão compacta, existe uma subsequência $\left\{u_{m_{j}}\right\}_{j \in \mathbb{N}} \subset\left\{u_{m}\right\}_{m \in \mathbb{N}}$ tal que $u_{m_{j}} \rightarrow u$ fortemente em $L^{p_{1}}(0, T ; E)$.

Teorema 1.1.12. Sejam $p_{0}>1$ e $E_{1}, E, E_{0}$ espaços de Banach. Se $E_{1} \stackrel{c}{\hookrightarrow} E \hookrightarrow E_{0}$, então $W_{\infty, p_{0}}\left(0, T ; E_{1}, E_{0}\right) \stackrel{c}{\hookrightarrow} C([0, T] ; E)$.

Demonstração: Ver Chepyzhov, V. V. and Vishik, M. I. [9], Apêndice A, página 345.

Lema 1.1.13. Sejam $X$ um espaço de Banach e $1 \leqslant p \leqslant \infty$. Se $u \in L^{p}(0, T ; X) e$ $\frac{d u}{d t} \in L^{p}(0, T ; X)$, então, após uma eventual modificação sobre um conjunto de medida nula de $(0, T), u:[0, T] \rightarrow X$ é contínua.

Demonstração: Ver Lions, J. L. [29], Capítulo 1, Lema 1.2, página 7.

Usaremos com frequência os conceitos de convergência fraca e fraca*. 
Definição 1.1.14. Sejam E um espaço de Banach $e\left\{u_{m}\right\}_{m \in \mathbb{N}} \subset$ E. Dizemos que $u_{m} \rightarrow u$ fracamente se $\left\langle u_{m}, \varphi\right\rangle \rightarrow\langle u, \varphi\rangle$, para toda $\varphi \in E^{\prime}$.

Definição 1.1.15. Sejam $E$ um espaço de Banach, $\varphi \in E^{\prime}$ e $\left\{\varphi_{m}\right\}_{m \in \mathbb{N}}$ é uma sequência de $E^{\prime}$. Dizemos que $\varphi_{m} \rightarrow \varphi$ fracamente $^{*}$ se $\left\langle u, \varphi_{m}\right\rangle \rightarrow\langle u, \varphi\rangle$, para todo $u \in E$.

As principais propriedades envolvendo as convergências fraca e fraca* estão reunidas na proposição abaixo, cuja demonstração pode ser vista em Robinson, J. C. [40], Seção 4.5, página 101.

Proposição 1.1.16. Sejam E um espaço de Banach, $\left\{u_{m}\right\}_{m \in \mathbb{N}}$ uma sequência de elementos de $E,\left\{\varphi_{m}\right\}_{m \in \mathbb{N}}$ uma sequência de elementos de $E^{\prime}, u \in E$ e $\varphi \in E^{\prime}$.

(i) Se $u_{m} \rightarrow u$ em E, então $u_{m} \rightarrow u$ em $E$;

(ii) Se $u_{m} \rightarrow$ em E, então $\left\|u_{m}\right\|_{E}$ é limitada e $\|u\|_{E} \leqslant \liminf \left\|u_{m}\right\|_{E}$;

(iii) Se $u_{m} \rightarrow u$ em $E$ e $\varphi_{m} \rightarrow \varphi$ em $E^{\prime}$, então $\left\langle u_{m}, \varphi_{m}\right\rangle \rightarrow\langle u, \varphi\rangle$;

(iv) $\operatorname{Se} \varphi_{m} \rightarrow \varphi$ em $E^{\prime}$, então $\varphi_{m} \stackrel{*}{\rightarrow} \varphi$ em $E^{\prime}$;

(v) Se $\varphi_{m} \stackrel{*}{\rightarrow} \varphi$ em $E^{\prime}$ e $u_{m} \rightarrow u$ em $E$, então $\left\langle u_{m}, \varphi_{m}\right\rangle \rightarrow\langle u, \varphi\rangle$;

(vi) Se E é um espaço de Hilbert, então $u_{m} \rightarrow u$ em $E$ se, e somente se, $u_{m} \rightarrow u$ em E e $\left\|u_{m}\right\|_{E} \rightarrow\|u\|_{E}$.

Se $1<p<\infty$, então os espaços $L^{p}(\Omega), W^{s, p}(\Omega), W_{0}^{s, p}(\Omega)$ são reflexivos e separáveis. A demonstração pode ser vista em Adams, R. [1]. Além disso, um dos resultados fundamentais da teoria dos espaços $L^{p}(0, T ; E)$ é aquele que estabelece a identificação entre os espaços duais $\left(L^{p}(0, T ; E)\right)^{\prime}=L^{q}\left(0, T ; E^{\prime}\right)$, onde $p$ e $q$ são expoentes conjugados, isto é, $\frac{1}{p}+\frac{1}{q}=1$. A demonstração desse fato é bastante sofisticada e será omitida. A dualidade entre esses espaços vem dada na forma integral por

$$
\langle u, v\rangle_{L^{q}(0, t ; E), L^{p}\left(0, T ; E^{\prime}\right)}=\int_{0}^{T}\langle u(t), v(t)\rangle_{E, E^{\prime}} d t .
$$

Com essa identificação os espaços $L^{p}(0, T ; E)$ com $1<p<\infty$ herdam as propriedades básicas do espaço de Banach $E$. Por exemplo, se $E$ é reflexivo, então $L^{p}(0, T ; E)$ será reflexivo e se $E$ for separável, então $L^{p}(0, T ; E)$ também será separável. Ver detalhes em Matos, M. [32].

Vamos agora dar uma condição suficiente para a convergência fraca em $L^{p}(\Omega)$, que pode ser encontrada no livro de Lions, J. L. [29], Capítulo 1, Lema 1.3, página 12.

Lema 1.1.17. Sejam $\mathcal{O}$ um domínio limitado em $\mathbb{R} \times \mathbb{R}^{n}$ e $1<q<\infty$. Se $\left\{g_{m}\right\}_{m \in \mathbb{N}}$ e g são funções de $L^{q}(\mathcal{O})$ tais que $\left\|g_{m}\right\|_{L^{q}(\mathcal{O})} \leqslant C$ e $g_{m} \rightarrow g$ quase sempre em $\mathcal{O}$, então $g_{m} \rightarrow g$ em $L^{q}(\mathcal{O})$. 
Sejam $E, E_{0}$ espaços de Banach satisfazendo $E \hookrightarrow E_{0}$. Em muitos casos a solução de uma equação de evolução pertence ao espaço $L^{p}(\tau, T ; E)$ e $\partial_{t} u \in L^{p_{1}}\left(\tau, T ; E_{0}\right)$. O próximo resultado será útil nesse contexto.

Teorema 1.1.18. Sejam E, $E_{0}$ espaços de Banach e suponha que $E \hookrightarrow E_{0}$. Se $u \in L^{\infty}(\tau, T ; E)$, $u(t) \in E_{0}$ para todo $t \in[\tau, T]$ e $\langle u(t), \varphi\rangle$ é uma função contínua em $t$ para todo $\varphi \in E_{0}^{\prime}$, isto é, $u:[\tau, T] \rightarrow E_{0}$ é fracamente contínua, então

(i) $u(t) \in E$ para $t \in[\tau, T],\|u(t)\|_{E} \leqslant\|u\|_{L^{\infty}(\tau, T ; E)}$ para todo $t \in[\tau, T]$ e $u:[\tau, T] \rightarrow E e^{\prime}$ fracamente continua;

(ii) a função $\|u(t)\|_{E} \quad e ́ \quad$ semicontínua inferiormente em $[\tau, T], \quad$ isto é, $\|u(t)\|_{E} \leqslant \liminf _{s \rightarrow t}\|u(s)\|_{E}$, para todo $t \in[\tau, T]$.

Demonstração: Ver Lions, J. L and Magenes, E. [30], Capítulo 8, Lema 8.1, página 275 ou Temam, R. [47], Capítulo 3, Lema 1.4, página 178.

Se $E$ e $F$ são espaços de Banach tais que $E \cap F \neq\{0\}$, o espaço $X=E \cap F$ pode ser munido da norma $\|\cdot\|_{X}=\|\cdot\|_{E}+\|\cdot\|_{F}$ e diremos que um conjunto é limitado em $X$ se for limitado em $E$ e em $F$.

O próximo resultado é o mesmo encontrado em Chepyzhov, V. V. and Vishik, M. I. [9], Teorema 1.8, página 33.

Lema 1.1.19. Sejam $p>1, q>1$ tais que $\frac{1}{p}+\frac{1}{q}=1$. Suponhamos que $H$ é um espaço de Hilbert e $V, E, X$ são espaços de Banach satisfazendo $V \hookrightarrow H \equiv H^{\prime} \hookrightarrow V^{\prime} \hookrightarrow X e$ $E \hookrightarrow H \equiv H^{\prime} \hookrightarrow E^{\prime} \hookrightarrow X$, onde $V^{\prime}$ e $E^{\prime}$ denotam os espaços duais de $V$ e E, respectivamente. Se $u \in L^{2}(0, T ; V) \cap L^{p}(0, T ; E)$ e a distribuição $\partial_{t} u$ pode ser representada como $\partial_{t} u(s)=w(s)+h(s)$, onde $w \in L^{2}\left(0, T ; V^{\prime}\right)$ e $h \in L^{q}\left(0, T ; E^{\prime}\right)$, então

(i) $u \in C([0, T] ; H)$;

(ii) a função $\|u(\cdot)\|_{H}^{2}$ é absolutamente contínua em $[0, T]$ e, além disso,

$$
\frac{d}{d t}\|u(t)\|_{H}^{2}=2\langle u(t), \dot{u}(t)\rangle=2\langle u(t), w(t)\rangle+2\langle u(t), h(t)\rangle
$$

para quase todo $t \in[0, T]$.

Demonstração: Ver Lions, J. L and Magenes, E. [30] (Capítulo 1, Teorema 3.1, página 19) e Temam, R. [47] (Capítulo 3, Lema 1.2, página 176), onde foi considerado o caso em que $V=E$ é um espaço de Hilbert e $p=2$. 
Citaremos algumas desigualdades que desempenham um papel muito importante no estudo das equações diferenciais parciais. As demonstrações podem ser encontradas em Brézis, H. [4].

Desigualdade de Young Sejam $p>1$ e $q$ o expoente conjugado de $p$, isto é, $\frac{1}{p}+\frac{1}{q}=1$. Se $a, b \in \mathbb{R}_{+}$, então

$$
a b \leqslant \frac{a^{p}}{p}+\frac{b^{q}}{q}
$$

Em particular, para todo $\epsilon>0$ existe $C_{\epsilon}=\frac{(\epsilon p)^{1-q}}{q}$ tal que

$$
a b \leqslant \epsilon a^{p}+C_{\epsilon} b^{q}
$$

Se $p=2$, então

$$
a b \leqslant \epsilon a^{2}+\frac{b^{2}}{4 \epsilon}
$$

Desigualdade de Hölder Sejam $1 \leqslant p \leqslant \infty$ e $q$ o expoente conjugado de $p$, isto é, $\frac{1}{p}+\frac{1}{q}=1$. Se $f \in L^{p}(\Omega)$ e $g \in L^{q}(\Omega)$, então $f g \in L^{1}(\Omega)$ e

$$
\int_{\Omega}|f g| d s \leqslant\|f\|_{p}\|g\|_{L^{q}}
$$

Desigualdade de Gronwall Sejam $y, a:\left[t_{0}, t_{1}\right] \rightarrow \mathbb{R}$ funções não negativas tais que $a, y \cdot a \in L^{1}\left(\left[t_{0}, t_{1}\right]\right)$ e existe uma constante $C$ satisfazendo

$$
y(t) \leqslant C+\int_{t_{0}}^{t} y(s) a(s) d s,
$$

então

$$
y(t) \leqslant C e^{\int_{t_{0}}^{t} a(s) d s}
$$

para $t \in\left[t_{0}, t_{1}\right]$.

Desigualdades diferenciais Sejam $a, h:\left[t_{0}, t_{1}\right] \rightarrow \mathbb{R}$ funções contínuas, $a(t) \geqslant 0$, $h(t) \geqslant 0$ e $y:\left[t_{0}, t_{1}\right] \rightarrow \mathbb{R}$ é uma função de classe $C^{1}$ tal que $y(t) \geqslant 0$. Se

$$
y^{\prime}(t) \leqslant a(t) y+h(t)
$$


então

$$
y(t) \leqslant y\left(t_{0}\right) e^{\int_{t_{0}}^{t} a(\tau) d \tau}+\int_{t_{0}}^{t} h(s) e^{\int_{s}^{t} a(\tau) d \tau} d s .
$$

Além disso, se $\gamma \geqslant 0$ e

$$
y^{\prime}(t)+\gamma y \leqslant h(t)
$$

então

$$
y(t) \leqslant y(0) e^{-\gamma t}+\int_{0}^{t} e^{-\gamma(t-s)} h(s) d s
$$

Os demais resultados dessa seção serão utilizados nas demonstrações dos teoremas de existência de solução fraca para $(0.1)$ e (0.10).

Definição 1.1.20. Seja $D \subset \mathbb{R}^{n+1}$ um subconjunto aberto. Dizemos que $F: D \rightarrow \mathbb{R}^{n}$ satisfaz as condições de Carathéodory em D quando

- $F(t, x)$ é mensurável em $t$, para cada $x$ fixo;

- $F(t, x)$ é contínua em $x$, para cada $t$ fixo;

- $S e C \subset D$ é um subconjunto compacto, então existe uma função real integrável $m_{C}(t)$ tal que $|F(t, x)| \leqslant m_{C}(t)$, para todo $(t, x) \in C$.

$$
\text { Sejam } a, b>0 \text { e } R=\left\{(t, x) \in \mathbb{R}^{n+1}:\left|t-t_{0}\right| \leqslant a,\left|x-x_{0}\right|_{\mathbb{R}^{n}} \leqslant b\right\} .
$$

Teorema 1.1.21. (Carathéodory) Se $F: R \rightarrow \mathbb{R}^{n}$ satisfaz as condições de Carathéodory em $R$, então existe $\beta>0$ e uma função $x:\left[t_{0}-\beta, t_{0}+\beta\right] \rightarrow \mathbb{R}^{n}$ absolutamente contínua satisfazendo

(i) $(t, x(t)) \in R$ para $t \in\left[t_{0}-\beta, t_{0}+\beta\right]$;

(ii) $x^{\prime}(t)=F(t, x)$ para $t \in\left[t_{0}-\beta, t_{0}+\beta\right]$, exceto num conjunto de medida nula; $e$ (iii) $x\left(t_{0}\right)=x_{0}$.

Demonstração: Ver Coddington, E. A. and Levinson, N. [18], Capítulo 2, Teorema 1.1, página 43. 
Definição 1.1.22. Dizemos que a função $x:\left[t_{0}-\beta, t_{0}+\beta\right] \rightarrow \mathbb{R}^{n}$ do Teorema 1.1.21 é uma solução no sentido estendido do problema de Cauchy

$$
\left\{\begin{array}{l}
x^{\prime}(t)=F(t, x) \\
x\left(t_{0}\right)=x_{0}
\end{array}\right.
$$

Teorema 1.1.23. (Prolongamento de solução) Sejam b>0, $0<T<+\infty$, $B=\left\{x \in \mathbb{R}^{n}:|x|_{\mathbb{R}^{n}} \leqslant b\right\},\left|x_{0}\right|_{\mathbb{R}^{n}} \leqslant b$ e $0<M<b$. Consideremos $D=[0, T] \times B$ e $F: D \rightarrow \mathbb{R}^{n}$ nas condições de Carathéodory. Se $x:\left[t_{0}-\beta, t_{0}+\beta\right] \rightarrow \mathbb{R}^{n}$ é uma solução no sentido estendido de

$$
\left\{\begin{array}{l}
x^{\prime}(t)=F(t, x) \\
x\left(t_{0}\right)=x_{0}
\end{array}\right.
$$

$e|x(t)|_{\mathbb{R}^{n}} \leqslant M$, para $t \in\left[t_{0}-\beta, t_{0}+\beta\right]$, então $x(t)$ tem um prolongamento em $[0, T]$.

Demonstração: Ver Coddington, E. A. and Levinson, N. [18], Capítulo 2, Teorema 1.3, página 47.

Teorema 1.1.24. (Banach-Alaoglu) Seja E um espaço de Banach separável. Se $u_{m}$ é uma sequência limitada em $E^{\prime}$, então $u_{m}$ tem uma subsequência convergente na topologia fraca* ${ }^{*}$

Demonstração: Ver Robinson, J. C. [40], Capítulo 4, Teorema 4.18, página 105.

Corolário 1.1.25. Seja E um espaço de Banach reflexivo. Se $u_{m}$ é uma sequência limitada em $E$, então $u_{m}$ tem uma subsequência que converge fracamente em E.

Demonstração: Ver Robinson, J. C. [40], Capítulo 4, Corolário 4.19, página 106.

Lema 1.1.26. Sejam $E$ e $F$ espaços de Banach. Se considerarmos o espaço $E \cap F$ com a norma $\|\cdot\|=\|\cdot\|_{E}+\|\cdot\|_{F}$, então o espaço dual de $E \cap F$ é $E^{\prime}+F^{\prime}$. Em particular, o espaço dual de $L^{2}(0, T ; E) \cap L^{p}(0, T ; F)$ é $L^{2}\left(0, T ; E^{\prime}\right)+L^{q}\left(0, T ; F^{\prime}\right)$ com $\frac{1}{p}+\frac{1}{q}=1$.

Demonstração: Ver Robinson, J. C. [40], Capítulo 8, página 231.

Teorema 1.1.27. (Desigualdades de Poincaré) Sejam $\Omega \subset \mathbb{R}^{n}$ um subconjunto aberto, conexo, limitado, com fronteira $\partial \Omega$ suave e $u \in H^{1}(\Omega)$. Se $\lambda_{1}$ é o menor autovalor positivo de $-\Delta$ (com as condições de fronteira apropriadas), então as seguintes Desigualdades de Poincaré se verificam 
(i) $\|\nabla u\|_{L^{2}(\Omega)}^{2} \geqslant \lambda_{1}\|u\|_{L^{2}(\Omega)}^{2}$, quando $u=0$ sobre $\partial \Omega$;

(ii) $\|\nabla u\|_{L^{2}(\Omega)}^{2} \geqslant \lambda_{1}\|u-\bar{u}\|_{L^{2}(\Omega)}^{2}$, quando $\frac{\partial u}{\partial \nu}=0$ sobre $\partial \Omega$, onde $\bar{u}=\frac{1}{|\Omega|} \int_{\Omega} u d x$.

Demonstração: Ver Smoller, J. [43], Capítulo 11, Teorema 11.11, página 112.

Lema 1.1.28. Se $u_{m} \rightarrow u$ em $L^{p}(\Omega), 1 \leqslant p<\infty$, então existe uma subsequência de $\left\{u_{m}\right\}$ que converge pontualmente para u quase sempre em $\Omega$.

Demonstração: Ver Robinson, J. C. [40], Capítulo 1, Corolário 1.12, página 27.

Teorema 1.1.29. Se $\Omega \subset \mathbb{R}^{n}$ é um subconjunto aberto, limitado e de classe $C^{\infty}$, então o problema de autovalor

$$
\left\{\begin{array}{l}
-\Delta u=\lambda u \quad \text { em } \Omega, \quad u \in W^{1,2}(\Omega), \\
\frac{\partial u}{\partial \nu}=0 \quad \text { sobre } \Gamma=\partial \Omega
\end{array}\right.
$$

possui uma quantidade enumerável de autovalores

$$
0=\lambda_{0} \leqslant \lambda_{1} \leqslant \lambda_{2} \leqslant \ldots \leqslant \lambda_{j} \leqslant \ldots
$$

tais que $\lambda_{j} \rightarrow+\infty$ e as autofunções $\left\{w_{j}\right\}_{j=0}^{\infty}$ satisfazem

$$
\frac{\partial w_{j}}{\partial \nu}=0 \quad \text { sobre } \Gamma=\partial \Omega
$$

e constituem um sistema ortonormal completo para $L^{2}(\Omega)$, isto é,

$$
v=\sum_{j=1}^{\infty}\left\langle v, w_{j}\right\rangle w_{j}
$$

para todo $v \in L^{2}(\Omega)$. Em particular,

$$
\|v\|_{L^{2}(\Omega)}^{2}=\sum_{j=1}^{\infty}\left\langle v, w_{j}\right\rangle_{L^{2}(\Omega)}^{2}
$$

Além disso, para todo $v \in W^{1,2}(\Omega)$ vale

$$
\|\nabla v\|_{L^{2}(\Omega)}^{2}=\sum_{j=1}^{\infty} \lambda_{j}\left\langle v, w_{j}\right\rangle_{L^{2}(\Omega)}^{2} .
$$

Demonstração: Ver Jost, J. [26], Capítulo 9, Teorema 9.5.2, página 260. 
Lema 1.1.30. Consideremos $p>2$ e $\Omega \subset \mathbb{R}^{n}$ um domínio suave. Se $E=L^{2}(\Omega), H^{1}(\Omega)$ ou $L^{p}(\Omega)$ e $P_{m}$ é o operador projeção ortogonal sobre o subespaço de $E$ gerado pelas $m$ primeiras autofunções do Laplaciano com condição de Neumann, então $\left\|P_{m} u\right\|_{E} \leqslant\|u\|_{E}$ e $P_{m} u \rightarrow u$ quando $m \rightarrow+\infty$.

Demonstração: Ver Robinson, J. C. [40], Capítulo 7, Lema 7.5, página 199.

\subsubsection{Os espaços $H^{s}(\Omega), H^{s}(\Gamma)$ e o traço da derivada normal}

Nessa subseção vamos dar uma caracterização dos espaços $H^{m}\left(\mathbb{R}^{n}\right), m \in \mathbb{N}$, que servirá de motivação para a definição dos espaços $H^{s}(\Omega)$ quando $s$ é um número real positivo e $\Omega \subset \mathbb{R}^{n}$ é um subconjunto aberto, limitado e com fronteira $\Gamma$ bem regular. A seguir, de posse dos espaços $H^{s}\left(\mathbb{R}^{n}\right)$ definiremos, via cartas locais, os espaços $H^{s}(\Gamma)$. Os resultados foram retirados do livro de Medeiros, L. A. and Milla Miranda, M. [35].

Sejam:

- $\mathcal{S}\left(\mathbb{R}^{n}\right)$, o espaço das funções $u \in C_{0}^{\infty}\left(\mathbb{R}^{n}\right)$ que decaem rapidamente no infinito.

- $\mathcal{S}^{\prime}\left(\mathbb{R}^{n}\right)$, o espaço das distribuições temperadas em $\mathbb{R}^{n}$ (espaço dual de $\mathcal{S}\left(\mathbb{R}^{n}\right)$ ).

Consideremos a função $J_{m}(x)=\left(1+|x|^{2}\right)^{\frac{m}{2}}, x \in \mathbb{R}^{n}$. Observemos que essa função é lentamente crescente no infinito. Vamos denotar por $\hat{u}$ a transformada de Fourier de $u$, isto é,

$$
\hat{u}(\xi)=(2 \pi)^{-\frac{n}{2}} \int_{\mathbb{R}^{n}} e^{-i x \cdot \xi} u(x) d x, \quad u \in \mathcal{S}\left(\mathbb{R}^{n}\right) ;
$$

e $\check{u}$ a transformada de Fourier inversa, isto é,

$$
\check{u}(\xi)=(2 \pi)^{-\frac{n}{2}} \int_{\mathbb{R}^{n}} e^{i x \cdot \xi} u(x) d x, \quad u \in \mathcal{S}\left(\mathbb{R}^{n}\right) .
$$

Definimos a transformada de Fourier de $u \in \mathcal{S}^{\prime}\left(\mathbb{R}^{n}\right)$ por

$$
\hat{u}(\phi):=u(\hat{\phi}), \quad \phi \in \mathcal{S}\left(\mathbb{R}^{n}\right) .
$$

Proposição 1.1.31. $H^{m}\left(\mathbb{R}^{n}\right)$ coincide com o conjunto $\left\{u \in \mathcal{S}^{\prime}\left(\mathbb{R}^{n}\right):\left(1+|x|^{2}\right)^{\frac{m}{2}} \hat{u} \in L^{2}\left(\mathbb{R}^{n}\right)\right\}$. Definindo

$$
\left.|||u|\right|_{m}=\left\|\left(1+|x|^{2}\right)^{\frac{m}{2}} \hat{u}\right\|_{L^{2}\left(\mathbb{R}^{n}\right)}
$$


a aplicação $u \mapsto\|\| u \|_{m}$ de $H^{m}\left(\mathbb{R}^{n}\right) \rightarrow \mathbb{R}_{+}$é uma norma equivalente a norma de Sobolev $\|u\|_{m}$.

Demonstração: Ver Medeiros, L. A. and Milla Miranda, M. [35], Capítulo II, Proposição 62, página 97.

Para todo $x \in \mathbb{R}^{n}$ e $s$ um número real não negativo, seja $J_{s}(x)=\left(1+|x|^{2}\right)^{\frac{s}{2}}$, semelhante a $J_{m}$ caso $m$ seja um número inteiro não negativo. Definimos $H^{s}\left(\mathbb{R}^{n}\right)$ como sendo o espaço vetorial

$$
H^{s}\left(\mathbb{R}^{n}\right)=\left\{u \in \mathcal{S}^{\prime}\left(\mathbb{R}^{n}\right):\left(1+|x|^{2}\right)^{\frac{s}{2}} \hat{u} \in L^{2}\left(\mathbb{R}^{n}\right)\right\}
$$

com o produto escalar definido por

$$
\langle u, v\rangle_{H^{s}\left(\mathbb{R}^{n}\right)}=\int_{\mathbb{R}^{n}}\left(1+|x|^{2}\right)^{s} \hat{u}(x) \hat{v}(x) d x,
$$

cuja norma por ele induzida é

$$
\|u\|_{H^{s}\left(\mathbb{R}^{n}\right)}^{2}=\int_{\mathbb{R}^{n}}\left(1+|x|^{2}\right)^{s}|\hat{u}(x)|^{2} d x .
$$

Esse espaço está imerso continuamente em $L^{2}\left(\mathbb{R}^{n}\right)$.

Proposição 1.1.32. $H^{s}\left(\mathbb{R}^{n}\right)$ é um espaço de Hilbert e $\mathcal{S}\left(\mathbb{R}^{n}\right)$ está imerso continuamente em $H^{s}\left(\mathbb{R}^{n}\right)$ e $\mathcal{S}\left(\mathbb{R}^{n}\right)$ é denso em $H^{s}\left(\mathbb{R}^{n}\right)$.

Demonstração: Ver Medeiros, L. A. and Milla Miranda, M. [35], Capítulo II, Proposição 63, página 99.

Corolário 1.1.33. $\mathcal{D}\left(\mathbb{R}^{n}\right)$ é denso em $H^{s}\left(\mathbb{R}^{n}\right)$ e $\mathcal{D}\left(\mathbb{R}^{n}\right) \hookrightarrow H^{s}\left(\mathbb{R}^{n}\right)$.

Demonstração: Ver Medeiros, L. A. and Milla Miranda, M. [35], Capítulo II, Corolário 64, página 100.

Seja $s \geqslant 0$ e $H^{-s}\left(\mathbb{R}^{n}\right)=\left(H^{s}\left(\mathbb{R}^{n}\right)\right)^{\prime}$ o espaço dual de $H^{s}\left(\mathbb{R}^{n}\right)$. Da Proposição 1.1.32 resulta que $\mathcal{S}\left(\mathbb{R}^{n}\right) \hookrightarrow H^{s}\left(\mathbb{R}^{n}\right) \hookrightarrow H^{-s}\left(\mathbb{R}^{n}\right) \hookrightarrow \mathcal{S}^{\prime}\left(\mathbb{R}^{n}\right)$. isto é,

Representamos por $\|f\|_{H^{-s}\left(\mathbb{R}^{n}\right)}$ a norma de uma aplicação linear contínua $f \in H^{-s}\left(\mathbb{R}^{n}\right)$,

$$
\|f\|_{H^{-s}\left(\mathbb{R}^{n}\right)}=\sup \left\{|\langle u, f\rangle|: u \in H^{s}\left(\mathbb{R}^{n}\right),\|u\|_{H^{s}\left(\mathbb{R}^{n}\right)}=1\right\}
$$


1 Resultados preliminares

Proposição 1.1.34. São verdadeiras as seguintes assertivas:

(a) $H^{-s}\left(\mathbb{R}^{n}\right)=\left\{f \in \mathcal{S}^{\prime}\left(\mathbb{R}^{n}\right):\left(1+|x|^{2}\right)^{-\frac{s}{2}} \hat{f} \in L^{2}\left(\mathbb{R}^{n}\right)\right\}$,

(b) $\|f\|_{H^{-s}\left(\mathbb{R}^{n}\right)}=\left\|\left(1+|x|^{2}\right)^{-\frac{s}{2}} \hat{f}\right\|_{L^{2}\left(\mathbb{R}^{n}\right)}$,

para toda $f \in H^{-s}\left(\mathbb{R}^{n}\right)$.

Demonstração: Ver Medeiros, L. A. and Milla Miranda, M. [35], Capítulo II, Proposição 65, página 101.

Indicamos por $H^{s}(\Omega), s$ um número real não negativo e $\Omega$ um subconjunto aberto do $\mathbb{R}^{n}$, ao espaço vetorial

$$
H^{s}(\Omega)=\left\{u=\left.v\right|_{\Omega}: v \in H^{s}\left(\mathbb{R}^{n}\right)\right\}
$$

Vamos munir $H^{s}(\Omega)$ de uma topologia. De fato, consideramos a aplicação linear sobrejetiva

$$
r: H^{s}\left(\mathbb{R}^{n}\right) \rightarrow H^{s}(\Omega),\left.\quad v \mapsto v\right|_{\Omega}
$$

Observemos que o núcleo $N(r)$ de $r$ é fechado. De fato, seja $\left\{v_{m}\right\}$ uma sequência de elementos de $H^{s}\left(\mathbb{R}^{n}\right)$ tal que $r v_{m}=0$ e $v_{m} \rightarrow v$ em $H^{s}\left(\mathbb{R}^{n}\right)$. Temos

$$
\left\|v_{m}-v\right\|_{H^{s}\left(\mathbb{R}^{n}\right)}^{2}=\int_{\mathbb{R}^{n}}\left(1+|x|^{2}\right)^{s}\left|\hat{v}_{m}(x)-\hat{v}(x)\right|^{2} d x \geqslant \int_{\mathbb{R}^{n}}\left|v_{m}(x)-v(x)\right|^{2} d x \geqslant \int_{\Omega}|v|^{2} d x
$$

$\log \mathrm{O}$

$$
\int_{\Omega}|v|^{2} d x \leqslant \lim _{m \rightarrow \infty}\left\|v_{m}-v\right\|_{H^{s}\left(\mathbb{R}^{n}\right)}^{2}=0,
$$

isto é, $\left.v\right|_{\Omega}=0$ o que mostra que $N(r)$ é fechado. Consideremos

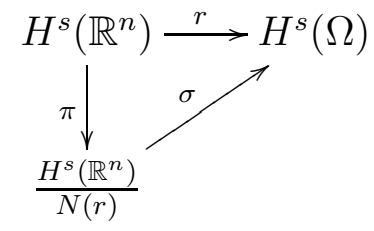

onde $\pi$ é o homomorfismo canônico. Temos que $\sigma$ é um isomorfismo entre espaços vetoriais e $X=\frac{H^{s}\left(\mathbb{R}^{n}\right)}{N(r)}$ é um espaço de Hilbert com produto escalar

$$
\langle[v],[w]\rangle_{X}=\langle[v],[w]\rangle_{1}+i\langle[v], i[w]\rangle_{1}
$$


onde

$$
\langle[v],[w]\rangle_{1}=4^{-1}\left(\|[v+w]\|^{2}-\|[v-w]\|^{2}\right) ;
$$

e norma

$$
\|[v]\|_{X}=\inf \left\{\|w\|_{H^{s}\left(\mathbb{R}^{n}\right)}: w \in[v]\right\} .
$$

Equipamos $H^{s}(\Omega)$ com a topologia dada por $\frac{H^{s}\left(\mathbb{R}^{n}\right)}{N(r)}$, via o isomorfismo $\sigma$. Assim,

$$
\begin{gathered}
\left\langle u_{1}, u_{2}\right\rangle_{H^{s}(\Omega)}=\left\langle\left[v_{1}\right],\left[v_{2}\right]\right\rangle_{X}, \quad \text { onde }\left.v_{1}\right|_{\Omega}=u_{1} \text { e }\left.v_{2}\right|_{\Omega}=u_{2}, \\
\|u\|_{H^{s}(\Omega)}=\|[v]\|_{X}=\inf \left\{\|v\|_{H^{s}\left(\mathbb{R}^{n}\right)}:\left.v\right|_{\Omega}=u\right\} .
\end{gathered}
$$

Com esse produto escalar, $H^{s}(\Omega)$ torna-se uma espaço de Hilbert.

Proposição 1.1.35. $\mathcal{D}(\bar{\Omega})$ é denso em $H^{s}(\Omega), s \in \mathbb{R}_{+}$.

Demonstração: Ver Medeiros, L. A. and Milla Miranda, M. [35], Capítulo II, Proposição 69, página 107.

No caso em que $s$ é um número inteiro não negativo $m$ e $\Omega$ um subconjunto aberto limitado, temos o próximo resultado.

Proposição 1.1.36. Se $\Omega \subset \mathbb{R}^{n}$ é um subconjunto aberto, limitado e de classe $C^{m}$, então

$$
H^{m}(\Omega)=\left\{u=\left.v\right|_{\Omega}: v \in H^{m}\left(\mathbb{R}^{n}\right)\right\}
$$

e as normas $\|u\|_{H^{m}(\Omega)}$ e \|\|$u\|\|_{H^{m}(\Omega)}$ são equivalentes, onde \|\|$u\|\|_{H^{m}(\Omega)}$ é a norma definida em (1.6) para $s=m$.

Demonstração: Ver Medeiros, L. A. and Milla Miranda, M. [35], Capítulo II, Proposição 70, página 107.

No que faremos a seguir, vamos considerar $\Omega=\mathbb{R}_{+}^{n}$ ou $\Omega$ um subconjunto aberto, limitado e bem regular do $\mathbb{R}^{n}$, isto é, $\Omega$ é de classe $C^{m}$ para todo $m=1,2, \ldots$, com fronteira $\Gamma$. Com $\mathcal{D}(\Gamma)$ representamos o espaço vetorial das funções reais $w$ definidas em $\Gamma$, possuindo derivadas parciais contínuas de todas as ordens. Vamos definir o espaço $H^{s}(\Gamma)$.

No caso em que $\Omega=\mathbb{R}_{+}^{n}$, tem-se $\Gamma=\left\{\left(x^{\prime}, 0\right): x^{\prime} \in \mathbb{R}^{n-1}\right\}$, identificando-se toda função $u$ definida em $\Gamma$ com a função $x^{\prime} \rightarrow u\left(x^{\prime}, 0\right)$ do $\mathbb{R}^{n-1}$ em $\mathbb{R}$. Com tal identificação 
temos que $\mathcal{D}(\Gamma)=\mathcal{D}\left(\mathbb{R}^{n-1}\right), L^{p}(\Gamma)=L^{p}\left(\mathbb{R}^{n-1}\right)$. Portanto, neste caso, definimos $H^{s}(\Gamma)$ como sendo $H^{s}\left(\mathbb{R}^{n-1}\right)$.

Suponhamos $\Omega$ um subconjunto aberto e limitado do $\mathbb{R}^{n}$, suposto bem regular. Consideremos um sistema de cartas locais de $\Gamma$, isto é, $\left\{\left(U_{1}, \varphi_{1}\right),\left(U_{2}, \varphi_{2}\right), \ldots,\left(U_{l}, \varphi_{l}\right)\right\}$, e funções testes $\sigma_{1}, \sigma_{2}, \ldots, \sigma_{l}$ no $\mathbb{R}^{n}$ tais que

$$
\operatorname{supp}\left(\sigma_{j}\right) \subset U_{j}, \quad j=1,2, \ldots, l, \quad \sum_{j=1}^{l} \sigma_{j}(x)=1, x \in \Gamma .
$$

Dada uma função $w$ definida em $\Gamma$, para todo $j=1,2, \ldots, l$ seja

$$
w_{j}(y)=\left\{\begin{array}{l}
\left(\sigma_{j} w\right)\left(\varphi_{j}^{-1}\left(y^{\prime}, 0\right)\right) \quad \text { se } y^{\prime} \in \Omega_{0}=(0,1)^{n-1} \\
0 \quad \text { se } y^{\prime} \in \mathbb{R}^{n-1} \backslash \Omega_{0}
\end{array}\right.
$$

Sendo $\operatorname{supp}\left(\Lambda_{0} \sigma_{j}\right)=\operatorname{supp} \sigma_{j} \cap \Gamma \subset U_{j} \cap \Gamma$ e como $\varphi_{j}$ aplica $U_{j} \cap \Gamma$ sobre $\Omega_{0} \times\{0\}$, temos

$$
\operatorname{supp}\left(w_{j}\right) \subset \varphi_{j}\left(\operatorname{supp} \sigma_{j} \cap \Gamma\right) \subset \Omega_{0} \times\{0\}
$$

Decorre daí que se $w \in \mathcal{D}(\Gamma)$, então $w_{j}$ pertence a $\mathcal{D}\left(\mathbb{R}^{n-1}\right)$ para todo $j=1,2, \ldots, l$.

Dado $s>0$ consideremos $H^{s}(\Gamma)$ como sendo o espaço vetorial das funções $w$ definidas em $\Gamma$ tais que $w_{j} \in H^{s}\left(\mathbb{R}^{n-1}\right)$ para todo $j=1,2, \ldots, l$, munido do seguinte produto escalar

$$
\langle w, v\rangle_{H^{s}(\Omega)}=\sum_{j=1}^{l}\left\langle w_{j}, v_{j}\right\rangle_{H^{s}\left(\mathbb{R}^{n-1}\right)}
$$

para todo par $w, v \in H^{s}(\Gamma)$. Temos que $H^{s}(\Gamma)$ é um espaço de Hilbert sendo $\mathcal{D}(\Gamma)$ denso em $H^{s}(\Gamma)$.

Consideremos agora $\Omega$ um aberto limitado do $\mathbb{R}^{n}$, com fronteira $\Gamma=\partial \Omega$ bem regular e seja $\nu$ a normal unitária exterior a $\Gamma$. Escolhemos um sistema de cartas locais $\left\{\left(U_{1}, \varphi_{1}\right), \ldots,\left(U_{l}, \varphi_{l}\right)\right\}$ de $\Gamma$ e funções testes do $\mathbb{R}^{n}, \sigma_{0}, \ldots, \sigma_{l}$ tais que

$$
\operatorname{supp}\left(\sigma_{0}\right) \subset \Omega, \quad \operatorname{supp}\left(\sigma_{j}\right) \subset U_{j}, \quad j=1,2, \ldots, l, \quad \sum_{j=1}^{l} \sigma_{j}(x)=1,
$$

para $x \in \bar{\Omega}$, de modo que para toda função $u$ definida em $\Gamma$ e $j=1, \ldots, l$ vale a seguinte relação

$$
\left(\frac{\partial u_{j}}{\partial \nu}\right)\left(\varphi^{-1}\left(y^{\prime}\right)\right)=-\frac{\partial}{\partial x_{n}}\left\{u_{j} \cdot \varphi_{j}^{-1}\right\}\left(y^{\prime}, 0\right), \quad y^{\prime} \in \Sigma_{0},
$$

onde $u_{j}=\sigma_{j} u$ e $\Sigma_{0}=\left\{\left(y^{\prime}, 0\right): 0 \leqslant y_{i} \leqslant 1, i=1, \ldots, n\right\}$.

Para todo $j=1, \ldots, m-1$ e $u \in \mathcal{D}(\bar{\Omega})$, seja $\Lambda_{j} u=\left.\frac{\partial^{j} u}{\partial \nu^{j}}\right|_{\Gamma}$ a derivada normal de ordem 
$j$ de $u$ e $\Lambda_{0} u=\left.u\right|_{\Gamma}$.

Teorema 1.1.37. Existe uma única aplicação linear e contínua $\Lambda$ do espaço $H^{m}(\Omega)$ sobre o espaço $\prod_{j=0}^{m-1} H^{m-j-\frac{1}{2}}$ com núcleo $\Lambda^{-1}(0)=H_{0}^{m}(\Omega)$, verificando a seguinte condição

$$
\Lambda u=\left(\Lambda_{0} u, \ldots, \Lambda_{m-1} u\right), \quad \forall u \in \mathcal{D}(\bar{\Omega})
$$

Tal aplicação admite uma inversa à direita linear e contínua.

Demonstração: Ver Medeiros, L. A. and Milla Miranda, M. [35], Capítulo II, Teorema 84, página 134.

Para finalizar essa subseção, vamos considerar $u$ num espaço conveniente, fixado posteriormente, de modo que o traço da derivada normal de $u$, isto é, o traço de $\frac{\partial u}{\partial \nu}$ pertence a um determinado espaço de Sobolev $H^{-s}(\Gamma), s>0$. Esse resultado é fundamental quando estudamos um problema de contorno cujo dado na fronteira é a derivada normal, como o problema de Neumann.

Observação 1.1.2. Quando consideramos $H^{s}(\Gamma), s>0$, como espaço vetorial normado real, representamos por $H^{-s}(\Gamma)$ o espaço dual forte de $H^{s}(\Gamma)$. No caso complexo, dado $f \in\left(H^{s}(\Gamma)\right)^{\prime}$, dual forte de $H^{s}(\Gamma)$, definimos o funcional conjugado $\bar{f}$ por $\langle\bar{f}, u\rangle=\overline{\langle f, u\rangle}$ para u em $H^{s}(\Gamma)$. Neste caso, consideramos como $H^{-s}(\Gamma)$ o espaço vetorial constituído das $\bar{f}$ tais que $f \in\left(H^{s}(\Gamma)\right)^{\prime}$, munido da norma:

$$
\|\bar{f}\|_{H^{-s}(\Gamma)}=\sup _{\|u\|=1}|\langle f, u\rangle|
$$

Temos que $L^{2}(\Gamma) \hookrightarrow H^{-s}(\Gamma)$, onde a inclusão é considerada no seguinte sentido: se $f \in L^{2}(\Gamma)$, identificamos $f$ ao funcional $\langle f, u\rangle=\langle f, u\rangle_{L^{2}(\Gamma)}$, onde $u \in H^{s}(\Gamma)$. Em particular, se $u \in \mathcal{D}(\bar{\Omega})$, então $\Lambda_{0} u$ e $\Lambda_{1} u=\left.\frac{\partial u}{\partial \nu}\right|_{\Gamma}$ podem ser considerados como vetores de $H^{-s}(\Gamma)$ para todo $s>0$.

Consideremos $\mathcal{H}^{1}(\Omega)=\left\{u \in H^{1}(\Omega): \Delta u \in L^{2}(\Omega)\right\}$ munido do produto interno $\langle u, v\rangle_{1}=\langle u, v\rangle_{H^{1}(\Omega)}+\langle\Delta u, \Delta v\rangle_{L^{2}(\Omega)}$ que o torna um espaço de Hilbert.

Proposição 1.1.38. A aplicação linear $\Lambda_{1}: \mathcal{D}(\bar{\Omega}) \rightarrow H^{-\frac{1}{2}}(\Gamma)$ definida por $u \mapsto \Lambda_{1} u=\left.\frac{\partial u}{\partial \nu}\right|_{\Gamma}$ se estende por continuidade a uma única aplicação linear e contínua

$$
\Lambda_{1}: \mathcal{H}^{1}(\Omega) \rightarrow H^{-\frac{1}{2}}(\Gamma)
$$

Demonstração: Ver Medeiros, L. A. and Milla Miranda, M. [35], Capítulo II, Proposição 91, página 140 . 
1 Resultados preliminares

Proposição 1.1.39. A aplicação $\tilde{\Lambda}_{1}: L^{2}\left(0, T ; \mathcal{H}^{1}(\Omega)\right) \rightarrow L^{2}\left(0, T ; H^{-\frac{1}{2}}(\Gamma)\right)$ é contínua.

Demonstração: Ver o artigo de Milla Miranda, M. [36].

\subsection{Coletânea de definições e resultados de Topologia}

Nessa seção faremos uma breve revisão de algumas definições e resultados básicos de topologia que serão úteis neste trabalho. As demonstrações podem ser encontradas em Kolmogorov, A. N. and Fomin, S. V. [28] e Schaefer, H. H. [41].

Axioma 1.2.1. (Primeiro axioma de enumerabilidade) Seja $\mathcal{T}$ um espaço topológico. Se todo ponto $x \in \mathcal{T}$ possui uma familia enumerável $U_{x}$ de vizinhanças tal que toda vizinhança $G$ de $x$ contém um elemento daquela família, então dizemos que $\mathcal{T}$ verifica o primeiro axioma de enumerabilidade ou que $\mathcal{T}$ tem uma base enumerável.

Teorema 1.2.2. Se $\mathcal{T}$ é um espaço topológico com base enumerável, então toda cobertura de um conjunto $X \subset \mathcal{T}$ constituída de conjuntos abertos contém uma subcobertura enumerável.

Definição 1.2.3. Um espaço topológico $\mathcal{T}$ é Fréchet-Urysohn se todo ponto aderente $x$ de um subconjunto $M \subset \mathcal{T}$ é limite de uma sequência de pontos $\left\{x_{m}\right\}_{m \in \mathbb{N}} \subset M$.

Axioma 1.2.4. (Axioma $T_{1}$, Primeiro axioma de separabilidade) Um espaço topológico $\mathcal{T}$ verifica o axioma $T_{1}$ se para quaisquer dois pontos distintos $x, y \in \mathcal{T}$ existe uma vizinhança $V_{x}$ de $x$ tal que y não pertence $a V_{x}$.

Axioma 1.2.5. (Axioma $T_{2}$, Segundo axioma de separabilidade) Um espaço topológico $\mathcal{T}$ verifica o axioma $T_{2}$ se para quaisquer dois pontos distintos $x, y \in \mathcal{T}$ existem vizinhanças $V_{x}$ e $V_{y}$ que não se interceptam, ou seja, $x \in V_{x}, y \in V_{y}$ e $V_{x} \cap V_{y}=\emptyset$.

Definição 1.2.6. Um espaço topológico que verifica o segundo axioma de enumerabilidade é denominado espaço de Hausdorff.

Definição 1.2.7. Um conjunto $\Sigma$ munido de uma relação $\leqslant$ é um conjunto dirigido se a relação $\leqslant$ satisfaz

(i) Se $x, y, z \in \Sigma$ são tais que $x \leqslant y$ e $y \leqslant z$, então $x \leqslant z$.

(ii) Para todo $x \in \Sigma, x \leqslant x$.

(iii) Para quaisquer $x, y \in \Sigma$ existe $z \in \Sigma$ tal que $x \leqslant z$ e $y \leqslant z$.

Definição 1.2.8. Uma rede num espaço topológico $\mathcal{T}$ é uma função $x: \Sigma \rightarrow \mathcal{T}$, onde $\Sigma$ é um conjunto dirigido. 
O resultado a seguir é a proposição 1.6.7 de Engelkin, R. [21], página 51.

Proposição 1.2.9. Um espaço topológico $\mathcal{T}$ é um espaço de Hausdorff se, e somente se, toda rede em $\mathcal{T}$ tem no máximo um limite.

Axioma 1.2.10. (Axioma de normalidade) Se um espaço topológico $\mathcal{T}$ é um espaço $T 1$ e quaisquer dois subconjuntos fechados disjuntos de $\mathcal{T}$ possuem vizinhanças que não se interceptam, então dizemos que $\mathcal{T}$ verifica o axioma de normalidade.

Definição 1.2.11. Um espaço topológico $\mathcal{T}$ é metrizável se sua topologia pode ser gerada por uma métrica.

Teorema 1.2.12. (Primeiro teorema da metrizabilidade de Urysohn) Um espaço topológico $\mathcal{T}$ com base enumerável é metrizável se, e somente se, é normal.

Definição 1.2.13. Um espaço topológico $\mathcal{T}$ é enumeravelmente compacto se todo subconjunto infinito de $\mathcal{T}$ tem um ponto de acumulação.

Teorema 1.2.14. Um espaço topológico $\mathcal{T}$ é enumeravelmente compacto se, e somente se, toda cobertura enumerável contém um subcobertura finita.

Teorema 1.2.15. Todo espaço de Hausdorff compacto é um espaço normal.

Teorema 1.2.16. (Segundo teorema da metrizabilidade de Urysohn) Um espaço de Hausdorff enumeravelmente compacto é metrizável se, e somente se, possui uma base topológica enumerável.

\subsection{Semigrupos em espaços topológicos e atratores}

Nesta seção daremos atenção aos resultados sobre a existência de atratores para semigrupos definidos num espaço de Hausdorff.

Definição 1.3.1. Seja $\mathcal{T}$ um espaço topológico. Um semigrupo $\{S(t), t \geqslant 0\}$ definido em $\mathcal{T}$ é uma família de aplicações $S(t): \mathcal{T} \rightarrow \mathcal{T}, t \geqslant 0$, satisfazendo

(i) $S\left(t_{1}+t_{2}\right)=S\left(t_{1}\right) S\left(t_{2}\right), t_{1} \geqslant 0, t_{2} \geqslant 0$;

(ii) $S(0)=I d$, onde Id é o operador identidade.

Assumimos daqui em diante que $\{S(t)\}$ é contínuo, ou seja, a aplicação $S(t)$ é contínua em $\mathcal{T}$, para todo $t \geqslant 0$.

Definição 1.3.2. Um conjunto $P \subset \mathcal{T}$ atrai um subconjunto $B \subset \mathcal{T}$ com relação a $\{S(t)\}$ se para toda vizinhança $V \supset P$ existe $T>0$ tal que $S(t) B \subset V$, para todo $t \geqslant T$. 
1 Resultados preliminares

Vamos definir os conjuntos $\omega$-limite em $\mathcal{T}$.

Definição 1.3.3. O conjunto $\omega$-limite de um subconjunto $B \subset \mathcal{T}$ é definido pela fórmula

$$
\omega(B)=\cap_{t \geqslant 0}{\overline{\cup_{h \geqslant t} S(h) B^{\mathcal{T}}}}^{\mathcal{T}}
$$

Proposição 1.3.4. O ponto $y \in \omega(B)$ se, e somente se, para toda vizinhança $V=V(y)$ de $y$ existem sequências $\left\{x_{n}\right\} \subseteq B$ e $\left\{t_{n}\right\} \subset \mathbb{R}_{+}, t_{n} \rightarrow+\infty$, tais que $S\left(t_{n}\right) x_{n} \in V$.

Demonstração: Ver Chepyzhov, V. V. and Vishik, M. I. [9], Proposição 2.1, página 215.

Proposição 1.3.5. Seja $\mathcal{T}$ um espaço de Hausdorff. Se K é um conjunto enumeravelmente compacto contido em $\mathcal{T}$ que atrai um subconjunto $B \subset \mathcal{T}$ com relação a $\{S(t)\}$, então

(i) $\omega(B) \neq \emptyset$,

(ii) $\omega(B)$ atrai $B$ com relação a $\{S(t)\}$.

Demonstração: Ver Chepyzhov, V. V. and Vishik, M. I. [9], Proposição 2.2, página 215.

Proposição 1.3.6. Assuma as hipóteses da Proposição 1.3.5. Se $K \subset \mathcal{T}$ é um subconjunto compacto, então:

(i) $\omega(B) \subset K$ é compacto;

(ii) $\omega(B)$ é o conjunto compacto minimal que atrai $B$ com respeito a $\{S(t)\}$, ou seja, todo conjunto compacto atrativo contém $\omega(B)$.

Demonstração: Ver Chepyzhov, V. V. and Vishik, M. I. [9], Proposição 2.3, página 216.

Vejamos agora a propriedade de invariância dos conjuntos $\omega$-limites.

Proposição 1.3.7. Sejam $\mathcal{T}$ um espaço de Hausdorff e $\{S(t)\}$ um semigrupo contínuo em $\mathcal{T}$. Se $K \subset \mathcal{T}$ é um subconjunto compacto que atrai $B \subset \mathcal{T}$ com relação ao semigrupo $\{S(t)\}$, então

$$
S(t) \omega(B)=\omega(B),
$$

para todo $t \geqslant 0$. 
Demonstração: Ver Chepyzhov, V. V. and Vishik, M. I. [9], Proposição 2.4, página 216.

Consideramos na próxima definição uma família $\mathcal{B}$ de subconjuntos de $\mathcal{T}$.

Definição 1.3.8. Um conjunto $K \subset \mathcal{T}$ atrai a família $\mathcal{B}$ com relação ao semigrupo $\{S(t)\}$ se $K$ atrai todo conjunto $B \in \mathcal{B}$.

Definição 1.3.9. $O$ conjunto $\mathcal{A}$ é o atrator da família $\mathcal{B}$ de subconjuntos de $\mathcal{T}$ se

(i) $\mathcal{A}$ é compacto na topologia de $\mathcal{T}$ e $\mathcal{A}$ atrai $\mathcal{B}$;

(ii) $\mathcal{A}$ é o conjunto compacto minimal que atrai $\mathcal{B}$ com respeito a $\{S(t)\}$, ou seja, todo conjunto compacto que atrai $\mathcal{B}$ contém $\mathcal{A}$.

Da definição acima segue que se o atrator existe, então ele é único.

Teorema 1.3.10. Sejam $\mathcal{T}$ um espaço topológico de Hausdorff e $\mathcal{B}$ uma família de subconjuntos de $\mathcal{T}$. Considere um semigrupo contínuo $\{S(t)\}$ definido em $\mathcal{T}$. Se existe um conjunto compacto $K$ que atrai $\mathcal{B}$ com relação a $\{S(t)\}$, então a família $\mathcal{B}$ tem um atrator $\mathcal{A}$.

Demonstração: Considere o conjunto

$$
\mathcal{A}={\overline{\cup_{B \subset \mathcal{B}} \omega(B)}}^{\mathcal{T}} \doteq{\overline{\mathcal{A}_{0}}}^{\mathcal{T}} .
$$

Da Proposição 1.3.5, o conjunto $\mathcal{A}$ atrai todo conjunto $B \in \mathcal{B}$. Além disso, pela Proposição 1.3.6, $\mathcal{A} \subseteq K, \mathcal{A}$ é compacto na topologia de $\mathcal{T}$ e é o conjunto compacto minimal que atrai $\mathcal{B}$. Portanto, $\mathcal{A}$ é o atrator.

Teorema 1.3.11. Sejam $\mathcal{T}$ um espaço de Hausdorff e $\{S(t)\}$ um semigrupo contínuo definido em $\mathcal{T}$. Se $K \subset \mathcal{T}$ é um subconjunto compacto que atrai uma família $\mathcal{B}$ de subconjuntos de $\mathcal{T}$, então o atrator $\mathcal{A}$ de $\mathcal{B}$ é estritamente invariante com relação a $\{S(t)\}$, isto é, $S(t) \mathcal{A}=\mathcal{A}$, para todo $t \geqslant 0$.

Demonstração: Considere o conjunto $\mathcal{A}_{0}=\cup_{B \in \mathcal{B}} \omega(B)$. Por (1.8), temos $S(t) \mathcal{A}_{0}=\mathcal{A}_{0}$ para todo $t \geqslant 0$. Além disso, $\mathcal{A}_{0} \subset K$ e portanto $K$ atrai $\mathcal{A}_{0}$. Considere o conjunto $\omega\left(\mathcal{A}_{0}\right)$. Pela Proposição 1.3.7, o conjunto $\omega\left(\mathcal{A}_{0}\right)$ é invariante, isto é, $S(t) \omega\left(\mathcal{A}_{0}\right)=\omega\left(\mathcal{A}_{0}\right)$ para todo $t \geqslant 0$. Pela definição de conjunto $\omega$-limite e por (1.9) temos

$$
\omega\left(\mathcal{A}_{0}\right)=\cap_{t \geqslant 0}{\overline{\cup_{h \geqslant t} S(h) \mathcal{A}_{0}}}^{\mathcal{T}}=\cap_{t \geqslant 0}{\overline{\mathcal{A}_{0}}}^{\mathcal{T}}={\overline{\mathcal{A}_{0}}}^{\mathcal{T}}=\mathcal{A} .
$$

Assim, $\mathcal{A}$ é estritamente invariante. 
1 Resultados preliminares

Corolário 1.3.12. Seja $\{S(t)\}$ um semigrupo contínuo definido num espaço Hausdorff compacto $\mathcal{T}$. Se $\mathcal{B}$ é a família de todos os subconjuntos de $\mathcal{T}$, então o atrator dessa família coincide com $\mathcal{A}=\omega(\mathcal{T})$.

\subsection{Aplicações para $(\mathcal{M}, \mathcal{T})$ - atratores}

Seja $\mathcal{T}$ um espaço topológico. Suponha que $d$ é uma métrica definida sobre $\mathcal{T}$ e denotemos por $\mathcal{M}$ o espaço métrico correspondente. Não assumimos que a métrica $d$ gera a topologia de $\mathcal{T}$. Essa seção trata da existência do $(\mathcal{M}, \mathcal{T})$ - atrator $\mathcal{A}$ de um semigrupo contínuo definido em $\mathcal{T}$, onde os conjuntos limitados de $\mathcal{T}$ serão determinados pela métrica $d$.

Definição 1.4.1. Seja $\mathcal{B}=B(\mathcal{M})$ a família de todos os subconjuntos de $\mathcal{T}$ limitados na métrica d. Um conjunto que atrai $B(\mathcal{M})$ é chamado $(\mathcal{M}, \mathcal{T})$ - atrativo e um atrator $\mathcal{A} d a$ família $B(\mathcal{M})$ é denominado $(\mathcal{M}, \mathcal{T})$-atrator.

Definição 1.4.2. Uma curva $\gamma(s), s \in \mathbb{R}$, em $\mathcal{T}$, é uma trajetória completa do semigrupo $\{S(t)\}$ se, para todo $t \geqslant 0, S(t) \gamma(s)=\gamma(t+s)$, para $s \in \mathbb{R}$. Uma trajetória completa $\gamma$ é limitada se o conjunto $B=\{\gamma(s): s \in \mathbb{R}\}$ é limitado em $\mathcal{M}$.

Definição 1.4.3. O núcleo $\mathcal{K}$ de $\{S(t)\}$ em $\mathcal{M}$ é a reunião de todas as trajetórias completas limitadas (em $\mathcal{M})$ de $\{S(t)\}$. A seção do núcleo no instante $t$ é dada por $\mathcal{K}(t)=\{\gamma(t): \gamma \in \mathcal{K}\}$.

Teorema 1.4.4. Seja $\{S(t)\}$ um semigrupo contínuo definido num espaço de Hausdorff $\mathcal{T}$ com um conjunto $(\mathcal{M}, \mathcal{T})$-atrativo $K$. Se $K$ é compacto na topologia de $\mathcal{T}$ e limitado em $\mathcal{M}$, então o semigrupo $\{S(t)\}$ tem um $(\mathcal{M}, \mathcal{T})$-atrator $\mathcal{A} \subseteq K e$

$$
\mathcal{A}=\mathcal{K}(0),
$$

onde $\mathcal{K}$ é o núcleo de $\{S(t)\}$ sobre $\mathcal{M}$.

Demonstração: Segue do Teorema 1.3 .10 que o $(\mathcal{M}, \mathcal{T})$-atrator $\mathcal{A}$ existe e $\mathcal{A} \subset K$. Do Teorema 1.3.11, $\mathcal{A}$ é estritamente invariante com respeito a $\{S(t)\}$, isto é, $S(t) \mathcal{A}=\mathcal{A}$, para $t \geqslant 0$. Vamos provar a igualdade (1.10). Considere uma trajetória completa limitada $\gamma \in \mathcal{K}$. O conjunto $B=\{\gamma(s): s \in \mathbb{R}\}$ é atraído por $K$, pois é limitado em $\mathcal{M}$. Além disso, $B$ é estritamente invariante, isto é, $S(t) B=B$, para todo $t \geqslant 0$, $\log 0 B \subset \omega(B)$ e assim $B \subset \mathcal{A}$. Portanto $\gamma(0) \in \mathcal{A}$, para todo $\gamma \in \mathcal{K}$ e $\mathcal{K}(0) \subset \mathcal{A}$.

Reciprocamente, seja $x \in \mathcal{A}$. Devido a propriedade de invariância estrita de $\mathcal{A}$, existe uma trajetória completa $\gamma(s) \in \mathcal{A}, s \in \mathbb{R}$, tal que $\gamma(0)=x$. O conjunto $\mathcal{A} \subset K$ é limitado em $\mathcal{M}$ e, portanto, $\gamma$ é uma trajetória completa limitada, ou seja, $x \in \mathcal{K}(0)$, para todo $x \in \mathcal{A}$. Finalmente, $\mathcal{K}(0) \supset \mathcal{A}$ e o teorema está provado. 


\section{Construção do atrator de trajetórias de equações de evolução abstratas}

O objetivo deste capítulo é dar um roteiro para a construção do atrator de trajetórias de equações de evolução abstratas. A principal referência é Chepyzhov, V. V. and Vishik, M. I. [9]

\subsection{Equações diferenciais ordinárias autônomas}

Nesta seção vamos construir o atrator de trajetórias de equações diferenciais ordinárias autônomas.

Consideremos um sistema de equações diferenciais ordinárias autônomas em $\mathbb{R}^{n}$ da forma

$$
\frac{d u(t)}{d t}=-F(u(t)), \quad t \geqslant 0
$$

onde $u=\left(u^{1}, \ldots, u^{n}\right) \in \mathbb{R}^{n}, F: \mathbb{R}^{n} \rightarrow \mathbb{R}^{n}$ é uma função contínua.

Assumimos que $F$ satisfaz uma condição de dissipatividade, isto é, existem constantes $C>0$ e $\delta>0$ tais que

$$
F(u) \cdot u=\sum_{i=1}^{N} F^{i}(u) u^{i} \geqslant-C+\delta|u|_{\mathbb{R}^{n}}^{2}
$$

para todo $u \in \mathbb{R}^{n}$.

Se o problema de Cauchy constituído da equação (2.1) e da condição inicial $\left.u\right|_{t=0}=u_{0}$ admite solução única, então o comportamento assintótico das soluções $u(t)$ de (2.1) pode ser estudado via o semigrupo solução $\left\{S(t): \mathbb{R}^{n} \rightarrow \mathbb{R}^{n}\right\}_{t \geqslant 0}, S(t) u_{0}=u(t)$. A unicidade de solução para o problema de Cauchy ocorre se a função vetorial $F: \mathbb{R}^{n} \rightarrow \mathbb{R}^{n}$ satisfaz uma condição de Lipschitz local. 
A abordagem acima não se aplica diretamente às equações cujo problema de Cauchy não tem unicidade de solução. Para contornar essa dificuldade usaremos o atrator de trajetórias.

Teorema 2.1.1. Se $u_{0} \in \mathbb{R}^{n}$, então existe uma função $u: \mathbb{R}_{+} \rightarrow \mathbb{R}^{n}$ de classe $C^{1}$ satisfazendo (2.1) e $u(0)=u_{0}$.

Demonstração: É consequência do Teorema de Peano.

Observemos que se $u:\left[0, T_{\max }\left[\rightarrow \mathbb{R}^{n}\right.\right.$ é solução maximal de (2.1) satisfazendo $u(0)=$ $u_{0}$, então da condição de dissipatividade (2.2) obtemos

$$
\frac{d}{d t}\left(\frac{1}{2}|u(t)|_{\mathbb{R}^{n}}^{2}\right)=\langle u(t), \dot{u}(t)\rangle=\langle u(t),-F(u(t))\rangle \leqslant C-\delta|u(t)|_{\mathbb{R}^{n}}^{2},
$$

donde concluímos que

$$
|u(t)|_{\mathbb{R}^{n}}^{2} \leqslant \frac{C}{\delta}+|u(0)|_{\mathbb{R}^{n}}^{2} e^{-2 \delta t}
$$

para $t \in\left[0, T_{\max }\left[\right.\right.$. Isso implica que $T_{\max }=+\infty \mathrm{e}$

$$
|u(t)|_{\mathbb{R}^{n}}^{2} \leqslant \frac{C}{\delta}+|u(0)|_{\mathbb{R}^{n}}^{2} e^{-2 \delta t}
$$

para todo $t \geqslant 0$.

No espaço $C^{1}\left(\mathbb{R}_{+} ; \mathbb{R}^{n}\right)$, consideremos o conjunto $\mathcal{K}^{+}$de todas as soluções da equação (2.1), isto é,

$$
\mathcal{K}^{+}=\left\{u \in C^{1}\left(\mathbb{R}_{+} ; \mathbb{R}^{n}\right): \frac{d u(t)}{d t}=-F(u(t)), \forall t \geqslant 0\right\} .
$$

Para $t \geqslant 0$, definamos $T(t): C^{1}\left(\mathbb{R}_{+} ; \mathbb{R}^{n}\right) \rightarrow C^{1}\left(\mathbb{R}_{+} ; \mathbb{R}^{n}\right)$ por $T(t) u(s)=u(t+s) . \mathrm{A}$ família $\{T(t), t \geqslant 0\}$ é um semigrupo e será denominado semigrupo translação.

Como a equação (2.1) é autônoma, o próximo resultado é imediato

Proposição 2.1.2. O conjunto $\mathcal{K}^{+}$é invariante com relação ao semigrupo $\{T(t), t \geqslant 0\}$, ou seja,

$$
T(t) \mathcal{K}^{+} \subseteq \mathcal{K}^{+}
$$

para todo $t \geqslant 0$.

Queremos construir o atrator do semigrupo $\{T(t), t \geqslant 0\}$ restrito ao espaço de trajetórias $\mathcal{K}^{+}$. Para isso, precisamos definir uma topologia no espaço $C^{1}\left(\mathbb{R}_{+} ; \mathbb{R}^{n}\right)$ que será 
denotada por $\Theta_{l o c}^{+}$.

Definição 2.1.3. No espaço $C^{1}\left(\mathbb{R}_{+} ; \mathbb{R}^{n}\right)$ definimos a topologia da convergência uniforme local, denotada por $\Theta_{\text {loc }}^{+}$e caracterizada da seguinte maneira: uma sequência $\left\{u_{m}\right\} \subset C^{1}\left(\mathbb{R}_{+} ; \mathbb{R}^{n}\right)$ converge para uma função $u \in C^{1}\left(\mathbb{R}_{+} ; \mathbb{R}^{n}\right)$ se

$$
\max _{s \in[0, T]}\left|u_{m}(s)-u(s)\right|_{\mathbb{R}^{n}}+\max _{s \in[0, T]}\left|u_{m}^{\prime}(s)-u^{\prime}(s)\right|_{\mathbb{R}^{n}} \rightarrow 0
$$

quando $m \rightarrow \infty$, para todo $T>0$.

Observação 2.1.1. O conjunto $\mathcal{K}^{+}$munido da topologia induzida dada na Definição 2.1.3 é denominado espaço de trajetórias da equação (2.1) e o Teorema 2.1.1 assegura que $\mathcal{K}^{+}$ é não vazio.

Exemplo 2.1.4. (i) Considere uma função $y: \mathbb{R}_{+} \rightarrow \mathbb{R}^{n}$ de classe $C^{1}$, satisfazendo $\lim _{s \rightarrow+\infty} y(s)=0$ e $\lim _{s \rightarrow+\infty} y^{\prime}(s)=0$. Se $y_{m}(s)=y(s+m)$ para $m \geqslant 1$, então $y_{m} \rightarrow 0$ em $\Theta_{l o c}^{+}$, quando $m \rightarrow+\infty$.

(ii) Seja y : $\mathbb{R}_{+} \rightarrow \mathbb{R}^{n}$ uma função de classe $C^{1}$ com suporte contido em [0,1]. Se $y_{m}(s)=y(s-m), m=1,2, \ldots$, então o suporte de $y_{m}$ está contido em $[m, m+1]$ e $y_{m} \rightarrow 0$ em $\Theta_{l o c}^{+}$, quando $m \rightarrow+\infty$.

Observação 2.1.2. A topologia $\Theta_{\text {loc }}^{+}$é metrizável por meio da métrica

$$
d(f, g)=\sum_{m=1}^{\infty} \frac{a_{m} d_{m}(f-g)}{1+d_{m}(f-g)}
$$

onde $d_{m}(f-g)=\max _{s \in\left[0, R_{m}\right]}|f(s)-g(s)|+\max _{s \in\left[0, R_{m}\right]}\left|f^{\prime}(s)-g^{\prime}(s)\right|,\left\{R_{m}\right\}$ é uma sequência não descrescente de números não negativos e $R_{m} \rightarrow+\infty$. Além disso, $\left\{a_{m}\right\}$ é uma sequência de números positivos satisfazendo $\sum_{m=1}^{\infty} a_{m}<+\infty$. O espaço $C^{1}\left(\mathbb{R}_{+} ; \mathbb{R}^{n}\right)$ munido dessa métrica é completo. Para a demonstração, veja Chepyzhov, V. V. and Vishik, M. I. [9], [12] páginas 98 e 927, respectivamente.

Para cada conjunto $K \subset C^{1}\left(\mathbb{R}_{+} ; \mathbb{R}^{n}\right)$ e para cada $T>0$, indicaremos por $\Pi_{[0, T]} K$ o conjunto das restrições das funções de $K$ ao intervalo $[0, T]$. Vamos formular um critério de compacidade em $\Theta_{l o c}^{+}$.

Proposição 2.1.5. $K \subset C^{1}\left(\mathbb{R}_{+} ; \mathbb{R}^{n}\right)$ é compacto em $\Theta_{l o c}^{+}$se, e somente se, $\Pi_{[0, T]} K$ é compacto em $C^{1}\left([0, T] ; \mathbb{R}^{n}\right)$, para todo $T>0$.

Demonstração: $(\Rightarrow)$ Se $\left\{u_{m}\right\} \subset K$ é uma sequência arbitrária, então, por hipótese, existe uma subsequência $\left\{u_{m, j}\right\} \subset\left\{u_{m}\right\}$ convergente. É claro que $\left\{\Pi_{[0, T]} u_{m, j}\right\}$ é uma subsequência de $\left\{\Pi_{[0, T]} u_{m}\right\}$ e é convergente. Como tomamos uma sequência arbitrária segue o resultado. $(\Leftarrow)$ Suponha que $\Pi_{[0, T]} K=\left\{\varphi:[0, T] \rightarrow \mathbb{R}^{n}:\right.$ existe $u \in K$ tal que $\left.\Pi_{[0, T]} u=\varphi\right\}$ é compacto, 
para todo $T>0$. Consideremos as sequências $\left\{u_{m}\right\} \subset K$ e $\left\{T_{m}\right\} \subset \mathbb{R} \operatorname{com} T_{m} \rightarrow+\infty$. Por hipótese, restrito ao segmento $\left[0, T_{1}\right]$, existe uma subsequência $\left\{u_{m, 1}\right\} \subset\left\{u_{m}\right\}$ que converge em $C^{1}\left(\left[0, T_{1}\right] ; \mathbb{R}^{n}\right)$. Analogamente, $\left\{u_{m, 1}\right\}$ contém uma subsequência $\left\{u_{m, 2}\right\}$ convergente em $C^{1}\left(\left[0, T_{2}\right] ; \mathbb{R}^{n}\right), T_{2}>T_{1}$. Repetindo esse processo para todo $T_{k}$ obtemos uma subsequência convergente $\left\{u_{m, k}\right\} \subset\left\{u_{m, k-1}\right\}$ em $C^{1}\left(\left[0, T_{k}\right] ; \mathbb{R}^{n}\right)$. Considere agora a subsequência diagonal $\left\{u_{m, m}: m=1,2, \ldots\right\}$ que é uma subsequência de toda sequência $\left\{u_{m, k}\right\}$ a partir de $k \geqslant m$. Temos que $\left\{u_{m, m}\right\}$ converge em $C^{1}\left(\left[0, T_{k}\right] ; \mathbb{R}^{n}\right)$ para todo $T_{k}>0$ e, portanto, $\left\{u_{m, m}\right\}$ é convergente em $C^{1}\left(\mathbb{R}_{+} ; \mathbb{R}^{n}\right)$.

Proposição 2.1.6. Se $\mathcal{K}^{+}$é o espaço de trajetórias de (2.1), então $\mathcal{K}^{+}$é fechado na topologia $\Theta_{\text {loc }}^{+}$. Portanto, $\mathcal{K}^{+}$é um espaço métrico completo.

Demonstração: Seja $\left\{u_{m}\right\} \subset \mathcal{K}^{+}$uma sequência tal que $u_{m} \rightarrow u$ em $\Theta_{l o c}^{+}$, isto é, para todo $T>0$, a sequência $u_{m} \rightarrow u$ uniformemente em $C^{1}\left([0, T] ; \mathbb{R}^{n}\right)$. Logo, em ambos os lados da equação $\frac{d u_{m}}{d t}(t)=-F\left(u_{m}(t)\right)$ podemos tomar o limite quando $m \rightarrow \infty$, donde obtemos $\frac{d u}{d t}(t)=-F(u(t))$. Assim, $u$ é uma solução de $(2.1)$ em $[0, T]$ e $u \in \mathcal{K}^{+}$. Portanto, $\mathcal{K}^{+}$é fechado.

Proposição 2.1.7. Para $t \geqslant 0, T(t):\left(C^{1}\left(\mathbb{R}_{+} ; \mathbb{R}^{n}\right), \Theta_{l o c}^{+}\right) \rightarrow\left(C^{1}\left(\mathbb{R}_{+} ; \mathbb{R}^{n}\right), \Theta_{\text {loc }}^{+}\right)$é contínuo.

Demonstração: Fixemos $t=h>0$. Suponhamos que $u_{m} \rightarrow u$ em $\Theta_{l o c}^{+}$, isto é, $u_{m} \rightarrow u$ em $C^{1}\left([0, T] ; \mathbb{R}^{n}\right)$, para todo $T>0$. Em particular, temos $u_{m} \rightarrow u$ em $C^{1}\left([0, h+T] ; \mathbb{R}^{n}\right)$ e em $C^{1}\left([h, h+T] ; \mathbb{R}^{n}\right)$, para todo $T>0$, ou seja, $T(h) u_{m}(s) \rightarrow T(h) u(s)$ em $\Theta_{l o c}^{+}$.

Vamos agora descrever os subconjuntos limitados $B$ de $\mathcal{K}^{+}$e estudar o comportamento assintótico dos conjuntos $T(t) B$ na topologia $\Theta_{l o c}^{+}$. Em $C^{1}\left(\mathbb{R}_{+} ; \mathbb{R}^{n}\right)$ consideremos o subespaço das funções limitadas, denotado por $C_{b}^{1}\left(\mathbb{R}_{+} ; \mathbb{R}^{n}\right)$ e munido da norma

$$
\|u\|_{C_{b}^{1}\left(\mathbb{R}_{+} ; \mathbb{R}^{n}\right)}=\sup _{s \geqslant 0}|u(s)|_{\mathbb{R}^{n}}+\sup _{s \geqslant 0}\left|u^{\prime}(s)\right|_{\mathbb{R}^{n}}
$$

O espaço $C_{b}^{1}\left(\mathbb{R}_{+} ; \mathbb{R}^{n}\right)$ munido da norma (2.6) é um espaço de Banach. Essa norma gera a topologia da convergência uniforme global sobre o semi-eixo $\mathbb{R}_{+}$e será usada para definir os conjuntos limitados de $\mathcal{K}^{+}$.

Proposição 2.1.8. O espaço de trajetórias $\mathcal{K}^{+}$está contido em $C_{b}^{1}\left(\mathbb{R}_{+} ; \mathbb{R}^{n}\right)$.

Demonstração: De (2.3) temos

$$
|u(t)|^{2} \leqslant|u(0)|^{2} e^{-2 \delta t}+\frac{C}{\delta}
$$


$\operatorname{logo} u \in C_{b}\left(\mathbb{R}_{+} ; \mathbb{R}^{n}\right)$. Como $F$ é contínua, segue que $F$ é limitada sobre todo conjunto limitado. Portanto, existe uma constante $C_{2}>0$ tal que

$$
\left|u^{\prime}(t)\right|^{2} \leqslant C_{2}
$$

para todo $t \geqslant 0$. Assim, $u \in C_{b}^{1}\left(\mathbb{R}_{+} ; \mathbb{R}^{n}\right)$ e $\mathcal{K}^{+} \subset C_{b}^{1}\left(\mathbb{R}_{+} ; \mathbb{R}^{n}\right)$.

Corolário 2.1.9. Se $C_{3}=\max \left\{|F(u)|_{\mathbb{R}^{n}}:|u|_{\mathbb{R}^{n}} \leqslant \frac{2 C}{\delta}\right\}$, então

$$
B_{0}=\left\{u \in C_{b}^{1}\left(\mathbb{R}_{+} ; \mathbb{R}^{n}\right):|u(s)|_{\mathbb{R}^{n}}^{2} \leqslant \frac{2 C}{\delta},\left|u^{\prime}(s)\right|_{\mathbb{R}^{n}}^{2} \leqslant C_{3} \text { para todo } s \geqslant 0\right\} \cap \mathcal{K}^{+}
$$

é um conjunto absorvente para o semigrupo $T(t)$ restrito a $\mathcal{K}^{+}$, ou seja, para todo subconjunto $B \subset \mathcal{K}^{+}$, existe $t_{0} \geqslant 0$ tal que $T(t) B \subset B_{0}$ quando $t \geqslant t_{0}$.

Demonstração: Se $P \subset \mathbb{R}^{n}$ é um subconjunto limitado, então existe $R>0$ tal que $\left|u_{0}\right| \leqslant R$, para todo $u_{0} \in P$. De (2.3) temos

$$
|u(t)|^{2} \leqslant e^{-2 \delta t}\left|u_{0}\right|^{2}+\frac{C}{\delta} \leqslant R^{2} e^{-2 \delta t}+\frac{C}{\delta} .
$$

Se $t_{0}=\frac{1}{2 \delta} \ln \frac{R^{2} \delta}{C}$ e $t \geqslant t_{0}$, então $|u(t)|^{2} \leqslant \frac{2 C}{\delta}$ e $\left|u^{\prime}(t)\right|^{2} \leqslant C_{3}$. Portanto, $B_{0}$ é um conjunto absorvente.

Proposição 2.1.10. Se $B_{0}$ é o conjunto definido no Corolário 2.1.9, então $B_{0}$ é compacto na topologia $\Theta_{l o c}^{+}$.

Demonstração: Pela Proposição 2.1.5, é suficiente provar que $\Pi_{[0, T]} B_{0}$ é compacto em $C^{1}\left([0, T] ; \mathbb{R}^{n}\right)$, para todo $T>0$. Se $\left\{u_{m}\right\} \subset B_{0}$, então essa sequência é uniformemente limitada em $C^{1}\left([0, T] ; \mathbb{R}^{n}\right)$. Logo, pelo Teorema de Arzelá-Ascoli, existe uma subsequência convergente $u_{m_{i}} \rightarrow u$ em $C\left([0, T] ; \mathbb{R}^{n}\right)$, onde $u \in C\left([0, T] ; \mathbb{R}^{n}\right)$. A função $u_{m_{i}}$ satisfaz a equação

$$
\frac{d u_{m_{i}}}{d t}(t)=-F\left(u_{m_{i}}(t)\right)
$$

para $t \in[0, T]$.

Como $F$ é contínua, segue que $F\left(u_{m_{i}}(t)\right) \rightarrow F(u(t))$ em $C\left([0, T] ; \mathbb{R}^{n}\right)$ e $\left\{\frac{d u_{m_{i}}}{d t}\right\}$ converge em $C\left([0, T] ; \mathbb{R}^{n}\right)$. Assim, $\left\{u_{m_{i}}\right\}$ converge em $C^{1}\left([0, T] ; \mathbb{R}^{n}\right)$ e $B_{0}$ é pré-compacto em $C^{1}\left([0, T] ; \mathbb{R}^{n}\right)$. Além disso, $B_{0}$ é fechado em $C^{1}\left([0, T] ; \mathbb{R}^{n}\right)$, pois $\mathcal{K}^{+}$é fechado na topologia $\Theta_{l o c}^{+}$. Portanto, $B_{0}$ é compacto na topologia $\Theta_{l o c}^{+}$. 
2 Construção do atrator de trajetórias de equações de evolução abstratas

Vamos definir o atrator de trajetórias do semigrupo translação $\{T(t)\}$ restrito ao espaço de trajetórias $\mathcal{K}^{+}$da equação $(2.1)$.

Definição 2.1.11. Um conjunto $\mathfrak{A} \subset \mathcal{K}^{+}$é o atrator de trajetórias da equação (2.1) se

(i) $\mathfrak{A}$ é compacto em $\Theta_{\text {loc }}^{+}$;

(ii) $\mathfrak{A}$ é estritamente invariante com respeito a $\{T(t)\}$, isto é, $T(t) \mathfrak{A}=\mathfrak{A}, t \geqslant 0$;

(iii) para todo conjunto $B \subset \mathcal{K}^{+}$temos $T(t) B \rightarrow \mathfrak{A}$ em $\Theta_{\text {loc }}^{+}$, ou seja,

$$
\operatorname{dist}_{C^{1}\left([0, T] ; \mathbb{R}^{n}\right)}(T(t) B, \mathfrak{A}) \rightarrow 0
$$

quando $t \rightarrow+\infty$, para todo $T>0$.

Teorema 2.1.12. Se a equação (2.1) verifica a condição de dissipatividade (2.2), então (2.1) possui um atrator de trajetórias $\mathfrak{A}$.

Demonstração: Consideremos o semigrupo $\{T(t)\}$ restrito ao conjunto $B_{0}$, dado em (2.8). Sabemos que a topologia $\Theta_{l o c}^{+}$é metrizável, o operador $T(t)$ é contínuo (Proposição 2.1.7) e, pela Proposição 2.1.10, $B_{0}$ é um espaço métrico compacto. Assim, pelo Teorema 1.3.10, $T(t)$ possui um atrator global $\mathfrak{A} \subset B_{0}$ que é compacto na topologia $\Theta_{l o c}^{+}$e estritamente invariante pelo semigrupo $\{T(t)\}$, isto é, $T(t) \mathfrak{A}=\mathfrak{A}, t \geqslant 0$.

Vamos verificar que $\mathfrak{A}$ é o atrator de trajetórias. Para isso, basta provar a propriedade de atração dada no item (iii) da Definição 2.1.11. Como $\mathfrak{A}$ é o atrator global de $\{T(t)\}$ definido sobre $B_{0}$, o conjunto $\mathfrak{A}$ atrai todo subconjunto limitado de $B_{0}$. Se $B \subset \mathcal{K}^{+}$, então, pelo Corolário 2.1.9, temos $T\left(t_{1}\right) B \subset B_{0}$ para $t_{1}$ suficientemente grande. Portanto,

$$
T(t) B=T\left(t-t_{1}+t_{1}\right) B=T\left(t-t_{1}\right) T\left(t_{1}\right) B \subseteq T\left(t-t_{1}\right) B_{0} \rightarrow \mathfrak{A}
$$

quando $t \rightarrow+\infty$. Assim, o teorema está provado.

O próximo passo é obter uma descrição aproximada do atrator de trajetórias $\mathfrak{A}$. Para isso, vamos introduzir o conceito de trajetória completa de (2.1). Uma função $u: \mathbb{R} \rightarrow \mathbb{R}^{n}$ de classe $C^{1}$ é uma trajetória completa de (2.1) se satisfaz

$$
\frac{d u(t)}{d t}=-F(u(t)), t \in \mathbb{R}
$$

Uma trajetória completa $u: \mathbb{R} \rightarrow \mathbb{R}^{n}$ é limitada se existe uma constante $C>0$ satisfazendo

$$
|u(t)| \leqslant C, \quad\left|u^{\prime}(t)\right| \leqslant C,
$$


para todo $t \in \mathbb{R}$, ou seja, $u \in C_{b}^{1}\left(\mathbb{R} ; \mathbb{R}^{n}\right)$. O conjunto de todas as trajetórias completas e limitadas da equação (2.1) é denominado núcleo de (2.1) e será denotado por $\mathcal{K}$, isto é,

$$
\mathcal{K}=\left\{u \in C_{b}^{1}\left(\mathbb{R} ; \mathbb{R}^{n}\right): u \text { satisfaz }(2.1)\right\}
$$

Definição 2.1.13. No espaço $C^{1}\left(\mathbb{R} ; \mathbb{R}^{n}\right)$ definimos a topologia $\Theta_{\text {loc }}$ da seguinte maneira: uma sequência $\left\{u_{m}\right\} \subset C^{1}\left(\mathbb{R} ; \mathbb{R}^{n}\right)$ converge para uma função $u \in C^{1}\left(\mathbb{R} ; \mathbb{R}^{n}\right)$ se

$$
\max _{s \in[-T, T]}\left|u_{m}(s)-u(s)\right|+\max _{s \in[-T, T]}\left|u_{m}^{\prime}(s)-u^{\prime}(s)\right| \rightarrow 0
$$

quando $m \rightarrow \infty$, para todo $T>0$.

O próximo teorema mostra que o conjunto $\mathcal{K}$ é não vazio e $\mathfrak{A}=\Pi_{+} \mathcal{K}$. Indicamos por $\Pi_{+} u$ a restrição da função $u: \mathbb{R} \rightarrow \mathbb{R}^{n}$ ao intervalo $[0,+\infty)$.

Teorema 2.1.14. Se as hipóteses do Teorema 2.1.12 estão satisfeitas, então $\mathfrak{A}=\Pi_{+} \mathcal{K}$. Além disso, $\mathcal{K} \subset C_{b}^{1}\left(\mathbb{R} ; \mathbb{R}^{n}\right)$ e $\mathcal{K}$ é compacto na topologia $\Theta_{\text {loc }}$.

Demonstração: Se $u \in \mathcal{K}$, então de (2.10) temos $\Pi_{+} u \in \mathcal{K}^{+}$e $\Pi_{+} T(t) u \in \mathcal{K}^{+}$, para todo $t \in \mathbb{R}$. Defina $B=\left\{\Pi_{+} T(t) u: t \in \mathbb{R}\right\} \subset \mathcal{K}^{+}$. Logo, $T(t) B \rightarrow \mathfrak{A}$ quando $t \rightarrow+\infty$. Como $B$ é estritamente invariante pelo semigrupo $\{T(t)\}$, isto é, $T(t) B=B, t \geqslant 0$, segue que $B \subset \mathfrak{A}$, pois $\mathfrak{A}$ é fechado. Assim, $\Pi_{+} \mathcal{K} \subset \mathfrak{A}$.

Reciprocamente, seja $u_{0} \in \mathfrak{A}$. Devido a invariância estrita de $\mathfrak{A}$, existe uma função $u_{1} \in \mathfrak{A}$ tal que $T(1) u_{1}(s)=u_{0}(s)$, isto é, $u_{1}(s+1)=u_{0}(s), s \geqslant 0$. Defina $\bar{u}(s)=u_{1}(s+1), s \geqslant-1$. É claro que $\bar{u}(t)$ satisfaz $(2.9)$ e $(2.10)$ para $t \geqslant-1$ e $\Pi_{+} \bar{u}(s)=$ $u_{0}(s)$.

Novamente pela invariância de $\mathfrak{A}$, existe $u_{2} \in \mathfrak{A}$ tal que $T(1) u_{2}(s)=u_{1}(s)$ e $T(2) u_{2}(s)=u_{0}(s), s \geqslant 0$. Defina $\bar{u}(s)=u_{2}(s+2), s \geqslant-2$. É claro que $\bar{u}(t)$ satisfaz $(2.9) \mathrm{e}$ (2.10) para $t \geqslant-2$. Repetindo esse processo obtemos uma função $\bar{u}(s), s \in \mathbb{R}$, satisfazendo (2.9) e (2.10) para todo $t \in \mathbb{R}$ e $\Pi_{+} \bar{u}(s)=u_{0}(s)$. Portanto $\mathfrak{A} \subseteq \Pi_{+} \mathcal{K}$.

Assim, a igualdade $\mathfrak{A}=\Pi_{+} \mathcal{K}$ está provada.

Segue de (2.8) e da construção feita acima que para $u \in \mathcal{K}$

$$
|u(t)|^{2} \leqslant \frac{2 C}{\delta}, \quad\left|u^{\prime}(t)\right|^{2} \leqslant C_{3}
$$

para todo $t \in \mathbb{R}$. Assim, $\mathcal{K} \subset C_{b}^{1}\left(\mathbb{R} ; \mathbb{R}^{n}\right)$. Além disso, $\mathcal{K}$ é compacto em $\Theta_{\text {loc }}$, pois $\mathfrak{A}$ é compacto em $\Theta_{l o c}^{+}$. 


\subsubsection{Exemplo 1}

Vamos descrever o atrator de trajetórias da equação diferencial

$$
u^{\prime}(t)=2 \sqrt{|u(t)|}(1-u(t))
$$

satisfazendo a condição inicial $u\left(t_{0}\right)=u_{0} \in \mathbb{R}$.

Afirmamos que a equação (2.12) verifica a condição de dissipatividade (2.2), ou seja, existem constantes $C>0$ e $\delta>0$ satisfazendo $-2 \sqrt{|u|} u+2 \sqrt{|u|} u^{2} \geqslant-C+\delta|u|^{2}$. De fato, basta tomarmos $C=20$ e $\delta=4$. Portanto, pelo Teorema 2.1.12, existe o atrator de trajetórias da equação (2.12).

Observemos que $u \equiv 0$ e $u \equiv 1$ são soluções de (2.12). De fato, $u(t) \equiv 0$ se $u_{0}=0$ e $u(t) \equiv 1$ é a única solução de (2.12) que satisfaz $u\left(t_{0}\right)=1$.

Se $0<u(t)<1$, então $u^{\prime}(t)>0$ para todo $t \in \mathbb{R}$ e resolvendo a equação (2.12), obtemos

$$
u(t)=\left(\frac{e^{2\left(t-t_{0}\right)}\left(1+\sqrt{u_{0}}\right)-\left(1-\sqrt{u_{0}}\right)}{e^{2\left(t-t_{0}\right)}\left(1+\sqrt{u_{0}}\right)+\left(1-\sqrt{u_{0}}\right)}\right)^{2} .
$$

Se $u_{0}=0$, então de (2.13) temos $u(t)=\left(\tanh \left(t-t_{0}\right)\right)^{2}$ e se $u_{0}=1$, segue que $u(t) \equiv 1, t \in \mathbb{R}$. É fácil ver que se $u_{0} \in(0,1)$, então não existe $\bar{t}$ satisfazendo $u(\bar{t})=1 \mathrm{e}$ $u\left(\overline{t_{1}}\right)=0$ se, e somente se, $\overline{t_{1}}=t_{0}+\frac{1}{2} \ln \left(\frac{1-\sqrt{u_{0}}}{1+\sqrt{u_{0}}}\right)$. Além disso, $\overline{t_{1}}<t_{0}$ e $\overline{t_{1}} \leqslant 0$ se, e somente se, $u_{0} \geqslant\left(\tanh t_{0}\right)^{2}$.

Portanto, se $u\left(t_{0}\right)=u_{0} \in(0,1)$, então

$$
u_{\delta}(t)=\left\{\begin{array}{l}
(\tanh (t-\delta))^{2} \quad \text { se } t>\delta \\
0 \text { se } t \leqslant \delta
\end{array}\right.
$$

onde $\delta=\overline{t_{1}}$ e $\lim _{t \rightarrow+\infty} u_{\delta}(t)=1$.

Assim, para cada $\delta$, a solução $u_{\delta}$ é uma translação da função

$$
u_{0}(t)=\left\{\begin{array}{l}
(\tanh t)^{2} \text { se } t>0 \\
0 \text { se } t \leqslant 0
\end{array}\right.
$$

Agora, se $u_{0}>1$, então $u(t)>1$ e, nesse caso, existe unicidade de solução. De (2.12) segue que $u(t)$ é decrescente e resolvendo a equação, obtemos $u(t)=\left(\frac{1+K e^{2\left(t-t_{0}\right)}}{1-K e^{2\left(t-t_{0}\right)}}\right)^{2}$, onde $K=\frac{\sqrt{u_{0}}-1}{\sqrt{u_{0}}+1}$ e o domínio da função é o intervalo $t \in\left(-\infty, t_{0}+\frac{1}{2} \ln \left(\frac{\sqrt{u_{0}}+1}{\sqrt{u_{0}}-1}\right)\right)$. 
Finalmente, se $u_{0}<0$, então $u(t)<0$. Resolvendo a equação diferencial, obtemos $u(t)=-\left(\frac{\sqrt{-u_{0}}-\tan \left(t-t_{0}\right)}{1+\sqrt{-u_{0}} \tan \left(t-t_{0}\right)}\right)^{2}$. Observemos que $u(\bar{t})=0$ se, e somente se, $\bar{t}=t_{0}+\arctan \sqrt{-u_{0}}$.

Portanto, se $u_{0}<0$, então

$$
u(t)=\left\{\begin{array}{l}
0 \text { se } t \geqslant t_{0}+\arctan \sqrt{-u_{0}} \\
-\left(\frac{\sqrt{-u_{0}}-\tan \left(t-t_{0}\right)}{1+\sqrt{-u_{0}} \tan \left(t-t_{0}\right)}\right)^{2} \text { se } t_{0}+\arctan \sqrt{-u_{0}}-\frac{\pi}{2}<t<t_{0}+\arctan \sqrt{-u_{0}} .
\end{array}\right.
$$

Se $\nu=t_{0}+\arctan \sqrt{-u_{0}}$, então podemos reescrever a solução (2.14) da seguinte forma

$$
u_{\nu}(t)=\left\{\begin{array}{l}
0 \text { se } t \geqslant \nu \\
-(\tan (t-\nu))^{2} \text { se } t<\nu
\end{array}\right.
$$

Resumindo o que dissemos, temos a figura abaixo

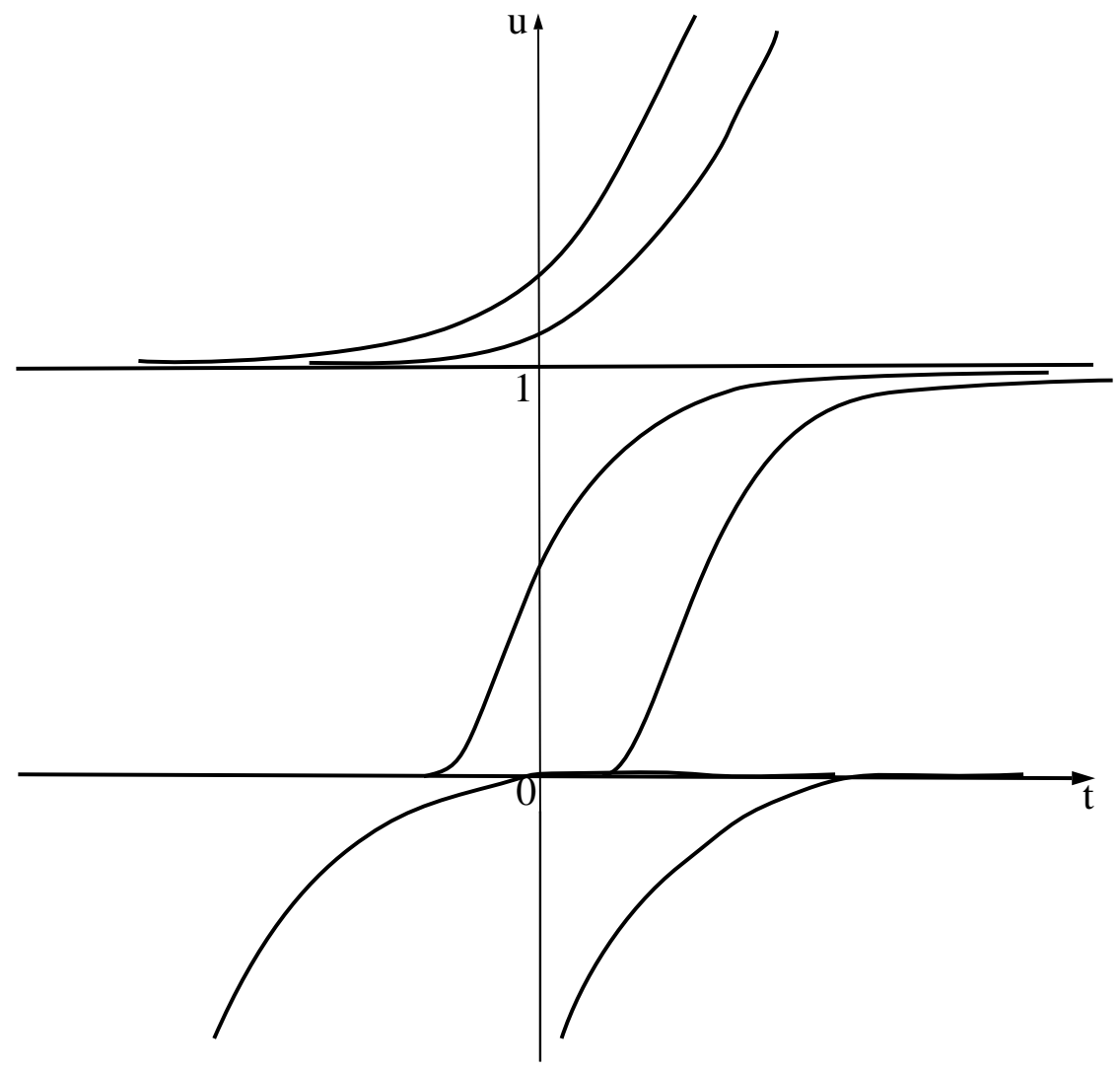

Figura 2.1: gráfico de $\mathrm{u}(\mathrm{t})$

Observemos que o problema de Cauchy para a equação (2.12) não tem unicidade de solução quando o dado inicial $u_{0} \in[0,1)$. Por definição, o espaço de trajetórias $\mathcal{K}^{+}$da 
equação (2.12) é um subconjunto de $C_{b}^{1}\left(\mathbb{R}_{+} ; \mathbb{R}\right)$, a saber

$$
\left\{u_{\delta}: \mathbb{R}_{+} \rightarrow \mathbb{R}: \delta=t_{0}+\frac{1}{2} \ln \left(\frac{1-\sqrt{u_{0}}}{1+\sqrt{u_{0}}}\right)\right\} \cup\{0\} \cup\{1\} .
$$

Se $F(u)=-2 \sqrt{|u(t)|}(1-u(t))$ e $C_{3}=\max \{|F(u)|:|u| \leqslant 1\}$, então, pelo Corolário 2.1.9, temos que

$$
B_{0}=\left\{u \in C_{b}^{1}\left(\mathbb{R}_{+} ; \mathbb{R}\right):|u|^{2} \leqslant 1,\left|u^{\prime}\right|^{2} \leqslant C_{3}\right\} \cap \mathcal{K}^{+}
$$

é um conjunto absorvente para o semigrupo translação $T(t)$ restrito a $\mathcal{K}^{+}$. E, pelo Teorema 1.3.11, o atrator de trajetórias da equação $(2.12)$ é $\mathfrak{A}=\omega\left(B_{0}\right)$.

\subsubsection{Exemplo 2: sistemas de primeira ordem}

Consideremos o sistema de equações diferenciais

$$
\frac{d u}{d t}=-L u-f(u)+g, t \geqslant 0
$$

onde $u \in \mathbb{R}^{n}, L$ é uma matriz real positiva de ordem $n, L \geqslant \delta_{0} I, \delta_{0}>0$ e $g$ é um vetor do $\mathbb{R}^{n}$. Assumimos que a função vetorial $f: \mathbb{R}^{n} \rightarrow \mathbb{R}^{n}$ é contínua (não supomos que $f$ satisfaz alguma condição de Lipschitz) e existe uma constante $C_{0}>0$ tal que

$$
f(u) \cdot u=\sum_{i=1}^{n} F^{i}(u) u^{i} \geqslant-C_{0}
$$

para todo $u \in \mathbb{R}^{n}$.

A equação (2.15) tem a forma de (2.1) com $F(u)=L u+f(u)-g$ e $F(u)$ verifica a condição de dissipatividade (2.2) para constantes $\delta>0$ e $C>0$ apropriadas. De fato, se $0<\epsilon<\delta_{0}$, então

$$
\begin{aligned}
F(u) \cdot u=L u \cdot u+f(u) \cdot u-g \cdot u & \geqslant \delta_{0}|u|^{2}-C_{0}-\epsilon|u|^{2}-\frac{|g|^{2}}{4 \epsilon} \\
& \geqslant\left(\delta_{0}-\epsilon\right)|v|^{2}-\left(C_{0}+\frac{1}{4 \epsilon}\right)|g|^{2} .
\end{aligned}
$$

Logo $\delta=\delta_{0}-\epsilon$ e $C=C_{0}+\frac{1}{4 \epsilon}$.

Portanto, dos Teoremas 2.1.12 e 2.1.14, a equação (2.15) tem um atrator de trajetórias $\mathfrak{A} \subset C_{b}^{1}\left(\mathbb{R}_{+} ; \mathbb{R}^{n}\right)$ e $\mathfrak{A}=\Pi_{+} \mathcal{K}$, onde $\mathcal{K}$ é o núcleo de $(2.15)$.

Se, além de contínua, admitimos que $f$ é localmente Lipschitz, então (2.15) com a condição inicial $u\left(t_{0}\right)=u_{0}$ tem solução única e podemos construir o semigrupo solução $\{S(t)\}$ 
atuando em $\mathbb{R}^{n}$, isto é, $S(t) u_{0}=u(t)$. Como $\{S(t)\}$ é contínuo e dissipativo, existe o atrator global $\mathcal{A}$ (ver Teorema 1.3.10). Pelo Teorema 1.4.4, temos a seguinte relação entre o atrator de trajetórias $\mathfrak{A}$ e o atrator global $\mathcal{A}$,

$$
\mathcal{A}=\{u(0): u \in \mathfrak{A}\}=\mathfrak{A}(0),
$$

onde $\mathfrak{A}(0)$ é a reunião dos valores de todas as funções $u \in \mathfrak{A}$ calculadas em $t=0$. Reciprocamente, o atrator de trajetórias $\mathfrak{A}$ consiste de todas as soluções de (2.15) que moram no atrator global, isto é, $\mathcal{A}$

$$
\mathfrak{A}=\left\{u(t)=S(t) u_{0}, t \geqslant 0: u_{0} \in \mathcal{A}\right\} .
$$

\subsubsection{Exemplo 3: sistemas de segunda ordem.}

Consideremos o sistema de equações diferenciais

$$
\frac{d^{2} u}{d t^{2}}+\gamma \frac{d u}{d t}=-L u-f(u)+g, t \geqslant 0
$$

onde $u \in \mathbb{R}^{n}, \gamma>0, L \geqslant \delta_{0} I$ é uma matriz real simétrica positiva (por exemplo, uma matriz diagonal) e $g \in \mathbb{R}^{n}$. Suponha que o campo vetorial $f$ é uma função potencial, isto é, existe uma função $\Phi: \mathbb{R}^{n} \rightarrow \mathbb{R}$ de classe $C^{1}$ tal que $f(u)=\nabla \Phi(u)$, para todo $u \in \mathbb{R}^{n}$. Assumimos que existem números reais positivos $m, C_{m}, \delta_{1}$, satisfazendo

$$
\begin{gathered}
\Phi(u) \geqslant-m|u|^{2}-C_{m}, \\
f(u) \cdot u-\delta_{1} \Phi(u)+m|u|^{2} \geqslant-C_{m},
\end{gathered}
$$

para todo $u \in \mathbb{R}^{n}$.

Seja $\alpha>0$ tal que $0<\alpha(\gamma-\alpha)<\delta_{0}$. Tomando o produto escalar em (2.17) por $\left(u^{\prime}+\alpha u\right)$, obtemos

$$
\begin{aligned}
\frac{1}{2} \frac{d}{d t}\left(\left|u^{\prime}+\alpha u\right|^{2}+\left|L^{\frac{1}{2}} u\right|^{2}+2 \Phi(u)\right) & +\alpha\left|L^{\frac{1}{2}} u\right|^{2}+(\gamma-\alpha)\left|u^{\prime}+\alpha u\right|^{2}-\alpha(\gamma-\alpha) u \cdot\left(u^{\prime}+\alpha u\right) \\
& =g \cdot\left(u^{\prime}+\alpha u\right)-\alpha f(u) \cdot u
\end{aligned}
$$

Usando a Desigualdade de Young (1.4) temos

$$
\alpha(\gamma-\alpha) u \cdot\left(u^{\prime}+\alpha u\right) \leqslant(\gamma-\alpha) \frac{\left|u^{\prime}+\alpha u\right|^{2}}{4}+(\gamma-\alpha) \alpha^{2}|u|^{2}
$$


e

$$
g \cdot\left(u^{\prime}+\alpha u\right) \leqslant(\gamma-\alpha) \frac{\left|u^{\prime}+\alpha u\right|^{2}}{4}+\frac{|g|^{2}}{\gamma-\alpha}
$$

Logo, de (2.19), (2.20), (2.21) e (2.22), obtemos

$$
\begin{aligned}
\frac{d}{d t}\left(\left|u^{\prime}+\alpha u\right|^{2}+\left|L^{\frac{1}{2}} u\right|^{2}+2 \Phi(u)\right) & +(\gamma-\alpha)\left|u^{\prime}+\alpha u\right|^{2}+2 \alpha\left|L^{\frac{1}{2}} u\right|^{2}-2(\gamma-\alpha) \alpha^{2}|u|^{2} \\
& \leqslant 2 \alpha\left(C_{m}-\delta_{1} \Phi(u)+m|u|^{2}\right)+\frac{2|g|^{2}}{\gamma-\alpha} \\
& \leqslant 2 \alpha C_{m}-2 \alpha \delta_{1} \Phi(u)+2 \alpha m|u|^{2}+\frac{2|g|^{2}}{\gamma-\alpha}
\end{aligned}
$$

Temos, por hipótese, $L \geqslant \delta_{0} I$ e se $\beta=\min \left\{\gamma-\alpha, \alpha \delta_{1}, 2 \alpha-\delta_{0}{ }^{-1}\left(2(\gamma-\alpha) \alpha^{2}+2 \alpha m\right)\right\}$, então de (2.23), resulta

$$
\frac{d}{d t}\left(\left|u^{\prime}+\alpha u\right|^{2}+\left|L^{\frac{1}{2}} u\right|^{2}+2 \Phi(u)\right)+\beta\left(\left|u^{\prime}+\alpha u\right|^{2}+\left|L^{\frac{1}{2}} u\right|^{2}+2 \Phi(u)\right) \leqslant \frac{2|g|^{2}}{\gamma-\alpha}+2 \alpha C_{m}
$$

Escolhendo $m$ tal que $m<\left(\delta_{0}-(\gamma-\alpha) \alpha\right)$ e definindo $z(t)=\left|u^{\prime}+\alpha u\right|^{2}+\left|L^{\frac{1}{2}} u\right|^{2}+2 \Phi(u)$, segue que

$$
\frac{d z}{d t}(t)+\beta z(t) \leqslant \frac{2|g|^{2}}{\gamma-\alpha}+2 \alpha C_{m}
$$

Portanto, temos

$$
z(t) \leqslant z(0) e^{-\beta t}+\left(\frac{2|g|^{2}}{\gamma-\alpha}+2 \alpha C_{m}\right) \beta^{-1}
$$

Observemos que

$$
z(t)=\left|u^{\prime}+\alpha u\right|^{2}+\left|L^{\frac{1}{2}} u\right|^{2}+2 \Phi(u) \geqslant\left|u^{\prime}+\alpha u\right|^{2}+\left(\delta_{0}-2 m\right)|u|^{2}-2 C_{m} .
$$

Se $0<m<\min \left\{\frac{\delta_{0}}{2},\left(\delta_{0}-(\gamma-\alpha) \alpha\right)\right\}$, então 


$$
\begin{aligned}
|u|^{2}+\left|u^{\prime}\right|^{2} & \leqslant \delta_{0}{ }^{-1}\left|L^{\frac{1}{2}} u\right|^{2}+\left(\left|u^{\prime}+\alpha u\right|+|\alpha u|\right)^{2} \\
& \leqslant 3\left|u^{\prime}+\alpha u\right|^{2}+\left(\frac{3 \alpha^{2}}{\delta_{0}}+\frac{1}{\delta_{0}}\right)\left|L^{\frac{1}{2}} u\right|^{2} \\
& \leqslant \max \left\{3, \frac{3 \alpha^{2}}{\delta_{0}}+\frac{1}{\delta_{0}}\right\} z(t) .
\end{aligned}
$$

Logo, se $k=\max \left\{3, \frac{3 \alpha^{2}}{\delta_{0}}+\frac{1}{\delta_{0}}\right\}$, então

$$
|u|^{2}+\left|u^{\prime}\right|^{2} \leqslant k\left(z(0) e^{-\beta t}+\left(\frac{2|g|^{2}}{\gamma-\alpha}+2 \alpha C_{m}\right) \beta^{-1}\right) .
$$

Considere $r=k\left(\frac{2|g|^{2}}{\gamma-\alpha}+2 \alpha C_{m}\right) \beta^{-1}$, segue que a bola $B_{0}=B(0,2 r)$ é um conjunto absorvente para (2.17), o que demonstra que o sistema (2.17) é dissipativo. Pelo Teorema 2.1.12, existe o atrator de trajetórias $\mathfrak{A}$ para (2.17) que, pelo Teorema 1.3.11, é dado por $\mathfrak{A}=\omega\left(B_{0}\right)$.

Observemos que o espaço de trajetórias $\mathcal{K}^{+}$para (2.17) é a reunião de todas as suas soluções satisfazendo $z(t) \leqslant 2 r$, onde $r=k\left(\frac{2|g|^{2}}{\gamma-\alpha}+2 \alpha C_{m}\right) \beta^{-1}$ e escolhemos $0<m<$ $\min \left\{\frac{\delta_{0}}{2},\left(\delta_{0}-(\gamma-\alpha) \alpha\right)\right\}$. Assim,

$\mathcal{K}^{+}=\left\{u \in C^{2}\left(\mathbb{R}_{+}, \mathbb{R}^{n}\right): \frac{d^{2} u}{d t^{2}}+\gamma \frac{d u}{d t}=-L u-f(u)+g\right.$, para todo $t \geqslant 0$ tal que $\left.z(t) \leqslant 2 r\right\}$.

Portanto, podemos enunciar o seguinte resultado

Proposição 2.1.15. Se as hipóteses (2.18) e (2.19) estão satisfeitas e $m$ é tal que $0<m<\min \left\{\frac{\delta_{0}}{2},\left(\delta_{0}-(\gamma-\alpha) \alpha\right)\right\}$, então (2.17) possui um atrator de trajetórias $\mathfrak{A}$.

O conjunto $\mathfrak{A}$ atrai os conjuntos limitados $B \subset \mathcal{K}^{+}$no seguinte sentido

$$
\operatorname{dist}_{C^{2}\left([0, T] ; \mathbb{R}^{n}\right)}(T(t) B, \mathfrak{A}) \rightarrow 0,
$$

para todo $T>0$.

Do Teorema 2.1.14, temos

$$
\mathfrak{A}=\Pi_{+} \mathcal{K}=\Pi_{+}\left\{u \in C_{b}^{2}\left(\mathbb{R} ; \mathbb{R}^{n}\right): u \text { é uma trajetória completa de }(2.17)\right\} \text {. }
$$

Se a função $f$ em (2.17) satisfaz uma condição de Lipschitz local, então temos a seguinte correspondência entre o atrator global $\mathcal{A}$ do semigrupo solução 
$\left\{S(t): u_{0} \rightarrow u(t): u_{0}\right.$ é um dado inicial $\}$ atuando em $\mathbb{R}^{2 n}$ e o atrator de trajetórias $\mathfrak{A}$ da equação $(2.17)$

$$
\begin{gathered}
\mathcal{A}=\left\{\left(u(0), \frac{d u}{d t}(0)\right): u \in \mathfrak{A}\right\}, \\
\mathfrak{A}=\left\{u: \mathbb{R}_{+} \rightarrow \mathbb{R}^{n}:\left(u(t), \frac{d u}{d t}(t)\right)=S(t)\left(u_{0}, v_{0}\right),\left(u_{0}, v_{0}\right) \in \mathcal{A}\right\} .
\end{gathered}
$$

\subsubsection{Dependência de um parâmetro}

Nesta seção vamos investigar a dependência do atrator de trajetórias em relação a um parâmetro $\epsilon \in \mathbb{R}$ pequeno.

Vamos estudar a equação

$$
\frac{d u}{d t}=-F(u, \epsilon), t \geqslant 0
$$

onde $\epsilon \in\left[-\epsilon_{0}, \epsilon_{0}\right]$. Assumimos que $F: \mathbb{R}^{n} \times\left[-\epsilon_{0}, \epsilon_{0}\right] \rightarrow \mathbb{R}^{n}$ é uma função contínua e satisfaz a seguinte condição de dissipatividade uniforme em relação a $\epsilon$ : existem constantes $C>0$ e $\delta>0$ tais que

$$
F(u, \epsilon) \cdot u \geqslant-C+\delta|u|^{2}
$$

para todo $u \in \mathbb{R}^{n}$ e para todo $\epsilon \in\left[-\epsilon_{0}, \epsilon_{0}\right]$.

Denotamos por $\mathcal{K}_{\epsilon}^{+}$o espaço de trajetórias da equação (2.27), dependendo de $\epsilon$ e contido em $C^{1}\left(\mathbb{R} ; \mathbb{R}^{n}\right)$. Pelos Teoremas 2.1 .12 e 2.1 .14 a equação (2.27) tem um atrator de trajetórias $\mathfrak{A}_{\epsilon}$ e vale a seguinte igualdade

$$
\mathfrak{A}_{\epsilon}=\Pi_{+} \mathcal{K}_{\epsilon},
$$

onde $\mathcal{K}_{\epsilon}=\left\{u \in C_{b}^{1}\left(\mathbb{R} ; \mathbb{R}^{n}\right): u\right.$ satisfaz $\left.\frac{d u}{d t}=-F(u, \epsilon), t \in \mathbb{R}\right\}$ é o núcleo da equação (2.27). Além disso, do Teorema 2.1.14, segue que $\mathcal{K}_{\epsilon}$ é compacto na topologia $\Theta_{\text {loc }}$ definida em 2.1.13.

Queremos estudar o comportamento de $\mathfrak{A}_{\epsilon}$ e $\mathcal{K}_{\epsilon}$ quando $\epsilon \rightarrow 0$.

Teorema 2.1.16. Se a condição de dissipatividade (2.28) é satisfeita, então $\mathcal{K}_{\epsilon} \rightarrow \mathcal{K}_{0}$ quando $\epsilon \rightarrow 0$, em $\Theta_{\text {loc }}$, isto é,

$$
\operatorname{dist}_{C^{1}\left([-T, T] ; \mathbb{R}^{n}\right)}\left(\mathcal{K}_{\epsilon}, \mathcal{K}_{0}\right) \rightarrow 0
$$

para todo $T>0$. 
Demonstração: A princípio observemos que $\mathcal{K}_{\epsilon}$ é uniformemente limitado em $C_{b}^{1}\left(\mathbb{R} ; \mathbb{R}^{n}\right)$, pois as constantes $C$ e $\delta$ em (2.28) não dependem de $\epsilon$ e, portanto, conseguimos limitar $|u(t)|$ e $\left|u^{\prime}(t)\right|$ por constantes que independem de $\epsilon$, exatamente como fizemos em (2.11).

Assumimos que a tese do enunciado é falsa, ou seja, existem $T_{1}>0, \sigma>0$ e sequências $\epsilon_{m} \rightarrow 0$ e $u_{m} \in \mathcal{K}_{\epsilon_{m}}$ tais que

$$
\operatorname{dist}_{C^{1}\left(\left[-T_{1}, T_{1}\right] ; \mathbb{R}^{n}\right)}\left(u_{m}, \mathcal{K}_{0}\right) \geqslant \sigma>0,
$$

para todo $m \in \mathbb{N}$. Como a sequência $\left\{u_{m}\right\}$ é uniformemente limitada, pelo Teorema de Arzelá-Ascoli, passando a uma subsequência (também denotada por $u_{m}$ ), podemos assumir que $u_{m} \rightarrow u_{0}$ em $C\left([-T, T] ; \mathbb{R}^{n}\right)$, para todo $T>0$. Observemos que a função $u_{0} \in C_{b}\left(\mathbb{R} ; \mathbb{R}^{n}\right)$ e $u_{m}$ verifica a equação

$$
\frac{d u_{m}}{d t}(t)=-F\left(u_{m}(t), \epsilon_{m}\right), t \in \mathbb{R} .
$$

Como $\mathcal{K}_{\epsilon_{m}}$ é compacto em $\Theta_{l o c}$ (Teorema 2.1.14) e $F$ é contínua em relação as variáveis $u$ e $\epsilon$, então fazendo $m \rightarrow \infty$ em (2.31), obtemos a igualdade

$$
\frac{d u_{0}}{d t}(t)=-F\left(u_{0}(t), 0\right), t \in \mathbb{R},
$$

de onde segue que $u_{0} \in C_{b}^{1}\left(\mathbb{R} ; \mathbb{R}^{n}\right)$. Consequentemente, $u_{0}$ é uma trajetória completa limitada da equação limite $\frac{d u}{d t}=-F(u, 0), t \in \mathbb{R}$, isto é, $u_{0} \in \mathcal{K}_{0}$. Assim,

$$
\operatorname{dist}_{C^{1}\left([-T, T] ; \mathbb{R}^{n}\right)}\left(u_{m}, \mathcal{K}_{0}\right) \leqslant \operatorname{dist}_{C^{1}\left([-T, T] ; \mathbb{R}^{n}\right)}\left(u_{m}, u_{0}\right) \rightarrow 0
$$

quando $m \rightarrow \infty$, o que contradiz (2.30) quando $T=T_{1}$.

Corolário 2.1.17. A família de atratores de trajetórias $\left\{\mathfrak{A}_{\epsilon}: \epsilon \in\left[0, \epsilon_{0}\right]\right\}$ é semicontínua superiormente em $\epsilon=0$ na topologia $\Theta_{\text {loc }}^{+}$, isto é,

$$
\operatorname{dist}_{C^{1}\left([0, T] ; \mathbb{R}^{n}\right)}\left(\mathfrak{A}_{\epsilon}, \mathfrak{A}_{0}\right) \rightarrow 0
$$

quando $\epsilon \rightarrow 0$, para todo $T>0$.

Demonstração: Segue do Teorema 2.1.16 e da igualdade (2.29).

Corolário 2.1.18. Suponha que a função $F(u, \epsilon)$ satisfaça uma condição de Lipschitz local em relação a variável $u$. Se $\mathcal{A}_{\epsilon}$ é o atrator global da equação (2.27), então a família de 
atratores $\left\{\mathcal{A}_{\epsilon}: \epsilon \in\left[0, \epsilon_{0}\right]\right\}$ é semicontínua superiormente em $\epsilon=0$, isto é,

$$
\operatorname{dist}_{\mathbb{R}^{n}}\left(\mathcal{A}_{\epsilon}, \mathcal{A}_{0}\right) \rightarrow 0
$$

quando $\epsilon \rightarrow 0$.

Demonstração: É consequência do Teorema 2.1.16 e do Corolário 2.1.17.

Exemplo 2.1.19. Consideremos a equação (2.15) com uma perturbação pequena no termo não-linear

$$
\frac{d u}{d t}=-L u-f(u)-\epsilon f_{1}(u)+g
$$

onde $\epsilon>0, f_{1}: \mathbb{R}^{n} \rightarrow \mathbb{R}^{n}$ é uma função contínua e existe uma constante $C_{1} \geqslant 0$, tal que $f_{1}(u) \cdot u \geqslant-C_{1}$, para todo $u \in \mathbb{R}^{n}$. Como no Exemplo 2.1.2, sejam $L \geqslant \delta_{0} I, \delta_{0}>0, g \in \mathbb{R}^{n}$ e $f$ é uma função contínua satisfazendo (2.16).

Afirmamos que existem constantes $C>0$ e $\delta>0$, tais que a equação (2.33) satisfaz a condição de dissipatividade (2.28). De fato, se $0<\epsilon_{1}<\delta_{0}$, então

$$
\begin{aligned}
\left(L u \cdot u+f(u) \cdot u+\epsilon f_{1}(u) \cdot u-g \cdot u\right) & \geqslant \delta_{0}|u|^{2}-C_{0}-\epsilon C_{1}-\epsilon_{1}|u|^{2}-4 \frac{|g|^{2}}{\epsilon_{1}} \\
& \geqslant\left(\delta_{0}-\epsilon_{1}\right)|u|^{2}-\left(C_{0}+C_{1} \epsilon+4 \frac{|g|^{2}}{\epsilon_{1}}\right) .
\end{aligned}
$$

Assim, $\delta=\delta_{0}-\epsilon_{1}$ e $C=C_{0}+C_{1} \epsilon+4 \frac{|g|^{2}}{\epsilon_{1}}$.

Pelo Teorema 2.1.12, a equação (2.33) tem um atrator de trajetórias $\mathfrak{A}_{\epsilon}$ e, pelo Corolário 2.1.17, temos $\mathfrak{A}_{\epsilon} \rightarrow \mathfrak{A}_{0}$ quando $\epsilon \rightarrow 0$, em $\Theta_{l o c}^{+}$.

Exemplo 2.1.20. Consideremos agora a equação (2.17) com o parâmetro $\epsilon$ aparecendo no termo que contém a derivada de maior ordem, isto é,

$$
\epsilon \frac{d^{2} u}{d t^{2}}+\gamma \frac{d u}{d t}=-L u-f(u)+g
$$

onde $\epsilon \in(0,1], \gamma>0$ e $f$ é uma função satisfazendo (2.18) e (2.19).

Seja $\alpha>0$, tal que $0<\alpha<\min \left\{\frac{\delta_{0}}{\gamma}, \gamma\right\}$.

Tomando o produto escalar em (2.34) por $\left(u^{\prime}+\alpha u\right)$, analogamente ao que fizemos no Exemplo 2.1.3, se definirmos $z_{\epsilon}(t)=\epsilon\left|u^{\prime}+\alpha u\right|^{2}+\left|L^{\frac{1}{2}} u\right|^{2}+2 \Phi(u)$ e assumirmos 


$$
\begin{gathered}
\beta=\min \left\{\gamma-\alpha, \alpha \delta_{1}, 2 \alpha\left(1-\frac{\gamma \alpha}{\delta_{0}}\right)-\frac{2 \alpha m}{\delta_{0}}\right\} \text { e } 0<m<\delta_{0}-\gamma \alpha, \text { então obteremos } \\
\frac{d}{d t} z_{\epsilon}(t)+\beta z_{\epsilon}(t) \leqslant \frac{2|g|^{2}}{\gamma-\alpha}+2 \alpha C_{m} .
\end{gathered}
$$

Portanto,

$$
\begin{aligned}
z_{\epsilon}(t) & \leqslant z_{\epsilon}(0) e^{-\beta t}+\left(\frac{2|g|^{2}}{\gamma-\alpha}+2 \alpha C_{m}\right) \beta^{-1} \\
& \leqslant z(0) e^{-\beta t}+\left(\frac{2|g|^{2}}{\gamma-\alpha}+2 \alpha C_{m}\right) \beta^{-1}
\end{aligned}
$$

Observemos que

$$
z_{\epsilon}(t)=\epsilon\left|u^{\prime}+\alpha u\right|^{2}+\left|L^{\frac{1}{2}} u\right|^{2}+2 \Phi(u) \geqslant \epsilon\left|u^{\prime}\right|^{2}+\left(\delta_{0}-2 m\right)|u|^{2}-2 C_{m}
$$

Se $0<m<\min \left\{\delta_{0}-\gamma \alpha, \frac{\delta_{0}}{2}, \frac{\delta_{0}}{\delta_{0}+1}\right\}$, ent $\tilde{a} O$

$$
|u|^{2}+\left|u^{\prime}\right|^{2} \leqslant \max \left\{3, \frac{3 \alpha^{2}}{\delta_{0}}+\frac{1}{\delta_{0}}\right\}\left(z(0) e^{-\beta t}+\left(\frac{2|g|^{2}}{\gamma-\alpha}+2 \alpha C_{m}\right) \beta^{-1}\right) .
$$

Observemos que as constantes que aparecem em (2.36) não dependem de $\epsilon$.

Consideremos $k=\max \left\{2, \frac{2 \alpha^{2}}{\delta_{0}}+\frac{1}{\delta_{0}}\right\}$ e $r=k\left(\frac{2|g|^{2}}{\gamma-\alpha}+2 \alpha C_{m}\right) \beta^{-1}$. De (2.36) segue que a bola $B_{0}=B(0,2 r)$ é um conjunto absorvente para (2.34). Pelo Teorema 2.1.12, a equação (2.34) tem um atrator de trajetórias $\mathfrak{A}_{\epsilon}$, para todo $\epsilon>0$.

Para $\epsilon=0$, obtemos o sistema de primeira ordem

$$
\gamma d_{t} u=-L u-f(u)+g
$$

Para (2.37), existem constantes $D>0$ e $\delta>0$, satisfazendo a condição de dissipatividade (2.28). De fato, se $\epsilon^{\prime}<\delta_{0}-m-\delta_{0} m$, então

$$
L u \cdot u+f(u) \cdot u-g \cdot u \geqslant\left(\delta_{0}-m-\delta_{0} m-\epsilon^{\prime}\right)|u|^{2}-\left(C_{m}+\delta_{0} C_{m}+\frac{4}{\epsilon^{\prime}}|g|^{2}\right) .
$$

Assim, escolhemos $D=C_{m}+\delta_{0} C_{m}+\frac{4}{\epsilon^{\prime}}|g|^{2}$ e $\delta=\delta_{0}-m-\delta_{0} m-\epsilon^{\prime}$.

Pelo Teorema 2.1.12, a equação (2.37) possui um atrator de trajetórias $\mathfrak{A}_{0}$.

Proposição 2.1.21. Se as hipóteses do Exemplo 2.1.20 são satisfeitas, então

$$
\mathfrak{A}_{\epsilon} \rightarrow \mathfrak{A}_{0}
$$


quando $\epsilon \rightarrow 0$, isto é,

$$
\operatorname{dist}_{C^{1}\left([0, T] ; \mathbb{R}^{n}\right)}\left(\mathfrak{A}_{\epsilon}, \mathfrak{A}_{0}\right) \rightarrow 0
$$

quando $\epsilon \rightarrow 0$, para todo $T>0$.

Demonstração: No Exemplo 2.1.20, determinamos a propriedade (2.36), onde as constantes independem de $\epsilon$. Suponhamos que (2.38) não seja verdade, isto é, existem $T_{1}>0$ e sequências $\epsilon_{j} \rightarrow 0$ e $\left\{u_{j}\right\} \subset \mathfrak{A}_{\epsilon_{j}}$ tais que

$$
\operatorname{dist}_{C^{1}\left(\left[0, T_{1}\right] ; \mathbb{R}^{n}\right)}\left(u_{j}, \mathfrak{A}_{0}\right) \geqslant k_{0}>0
$$

para todo $j \in \mathbb{N}$.

De (2.36), temos que a sequência $\left\{u_{j}\right\}$ é uniformemente limitada no espaço $C_{b}^{1}\left(\mathbb{R}_{+} ; \mathbb{R}^{n}\right)$, então, do Teorema de Arzelá, passando a uma subsequência (também denotada por $u_{j}$ ) podemos assumir que $u_{j} \rightarrow u_{0}$ em $C\left([0, T] ; \mathbb{R}^{n}\right)$, para todo $T>0$. Além disso $u_{0} \in C_{b}\left([0, T] ; \mathbb{R}^{n}\right)$.

As funções $u_{j}$ satisfazem a seguinte igualdade

$$
\epsilon_{j} \frac{d^{2} u_{j}}{d t^{2}}+\gamma \frac{d u_{j}}{d t}=-L u_{j}-f\left(u_{j}\right)+g
$$

Observemos que a função $f$ é contínua na variável $u$ e $u_{j} \in C_{b}^{2}\left(\mathbb{R}_{+}, \mathbb{R}^{n}\right)$. Logo fazendo $j \rightarrow+\infty$ em (2.40), obtemos a igualdade

$$
\gamma \frac{d u_{0}}{d t}=-L u_{0}-f\left(u_{0}\right)+g
$$

Assim, $u_{0} \in C_{b}^{1}\left(\mathbb{R}_{+} ; \mathbb{R}^{n}\right)$ e $u_{0}$ mora no atrator de trajetórias $\mathfrak{A}_{0}$ da equação limite (2.37). Além disso, $u_{0}$ é o limite da sequência $\left\{u_{j}\right\}$ em $C^{1}\left([0, T] ; \mathbb{R}^{n}\right)$, para todo $T>0$.

Portanto,

$$
\operatorname{dist}_{C^{1}\left([0, T] ; \mathbb{R}^{n}\right)}\left(u_{j}, \mathfrak{A}_{0}\right) \leqslant \operatorname{dist}_{C^{1}\left([0, T] ; \mathbb{R}^{n}\right)}\left(u_{j}, u_{0}\right) \rightarrow 0
$$

o que contradiz (2.39) quando $T=T_{1}$.

Concluimos que a relação (2.38) é válida. 


\subsection{Equações de evolução abstratas}

Nesta seção vamos esboçar o roteiro para a construção do atrator de trajetórias de equações de evolução abstratas. Não assumimos unicidade de solução para o problema de Cauchy correspondente.

Sejam $E_{1}$ e $E_{0}$ espaços de Banach tais que $E_{1} \subset E_{0}$ e $A: E_{1} \rightarrow E_{0}$ é um operador diferencial não linear. Consideremos uma equação de evolução autônoma abstrata da forma

$$
\partial_{t} u=A(u), t \geqslant 0 .
$$

Dado um espaço de Banach $E$ satisfazendo $E_{1} \subset E \subset E_{0}$ e $T>0$, indicamos por $\mathcal{F}(0, T ; E)$ um conjunto das funções $u:[0, T] \rightarrow E$ e suponha que $\mathcal{F}(0, T ; E)$ é um espaço de Banach. Assumimos que se $u \in \mathcal{F}(0, T ; E)$, então a função $A(u(t))$ pertence ao espaço de Banach $\mathcal{F}\left(0, T ; E_{0}\right)=\left\{v:[0, T] \rightarrow E_{0}\right\}$, para $0 \leqslant t \leqslant T$, onde $\mathcal{F}(0, T ; E) \subset \mathcal{F}\left(0, T ; E_{0}\right)$. Assumimos também que a derivada $\partial_{t} u(t)$ é no sentido de distribuição com valores em $E_{0}$, com $0 \leqslant t \leqslant T$, isto é, $\partial_{t} u \in \mathcal{D}^{\prime}\left((0, T) ; E_{0}\right)$ e $\mathcal{F}\left(0, T ; E_{0}\right) \subset \mathcal{D}^{\prime}\left((0, T) ; E_{0}\right)$.

Um exemplo para o espaço $\mathcal{F}(0, T ; E)$ é dado por $\mathcal{F}(0, T ; E)=C([0, T] ; E)=$ $\left\{u:[0, T] \rightarrow E: u\right.$ é contínua\}. Outros exemplos são $\mathcal{F}(0, T ; E)=L^{p}(0, T ; E)=$ $\left\{u:[0, T] \rightarrow E: \int_{0}^{T}\|u(s)\|_{E}^{p}<+\infty\right\}$, onde $1 \leqslant p<+\infty$, e $\mathcal{F}(0, T ; E)=L^{\infty}(0, T ; E)$.

Definição 2.2.1. Uma função $u \in \mathcal{F}(0, T ; E)$ é solução fraca de (2.42) no intervalo $[0, T]$, quando a igualdade $\partial_{t} u(t)=A(u(t))$ é satisfeita no sentido de distribuição em $\mathcal{D}^{\prime}\left((0, T) ; E_{0}\right)$.

Consideremos o conjunto

$$
\mathcal{F}_{\text {loc }}^{+}=\left\{u: \mathbb{R}_{+} \rightarrow E: \Pi_{[0, T]} u \in \mathcal{F}(0, T ; E) \text {, para todo } T>0\right\},
$$

onde $\Pi_{[0, T]}$ é o operador restrição ao intervalo $[0, T]$.

Definição 2.2.2. Uma função $u \in \mathcal{F}_{\text {loc }}^{+}$é solução fraca global de (2.42) se a função $\Pi_{[0, T]} u \in$ $\mathcal{F}(0, T ; E)$ é solução fraca de (2.42) no intervalo $[0, T]$, para todo $T>0$.

Vamos denotar por $\mathcal{K}^{+}$o conjunto de soluções fracas globais de (2.42), onde $\mathcal{K}^{+} \subset \mathcal{F}_{\text {loc }}^{+}$. Os elementos de $\mathcal{K}^{+}$são denominados trajetórias.

Definimos o operador $T(t): \mathcal{F}_{l o c}^{+} \rightarrow \mathcal{F}_{l o c}^{+}$por

$$
T(t) u(s)=u(t+s),
$$

para todo $s \geqslant 0$ e $t \geqslant 0$. Assumimos que $T(t)$ aplica uma função de $\mathcal{F}(0, T ; E)$ num elemento de $\mathcal{F}(0, T-t ; E)$ e $\|T(t) u\|_{\mathcal{F}(0, T-t ; E)} \leqslant\|u\|_{\mathcal{F}(0, T ; E)}$, para todo $T \geqslant t \geqslant 0$. 
A família de operadores $\left\{T(t): \mathcal{F}_{l o c}^{+} \rightarrow \mathcal{F}_{l o c}^{+}: t \geqslant 0\right\}$ forma um semigrupo, isto é,

$$
T(0)=I, \quad T\left(t_{1}\right) T\left(t_{2}\right)=T\left(t_{1}+t_{2}\right), \forall t_{1}, t_{2} \geqslant 0
$$

Assumimos que o conjunto $\mathcal{K}^{+}$satisfaz a seguinte propriedade

$$
T(t) \mathcal{K}^{+} \subset \mathcal{K}^{+}
$$

para todo $t \geqslant 0$, isto é, se $u$ é uma solução fraca global de (2.42), então $T(t) u$ também é uma solução fraca global de (2.42). Essa propriedade é naturalmente satisfeita quando lidamos com equações autônomas.

Estamos interessados em estudar a propriedade de atração de uma família de trajetórias contidas em $\mathcal{K}^{+}$pela ação do semigrupo $\{T(t)\}$ quando $t \rightarrow+\infty$. Mas antes, definiremos uma topologia no conjunto $\mathcal{F}_{\text {loc }}^{+}$.

Indicamos por $\Theta_{[0, T]}$ a topologia definida no espaço $\mathcal{F}(0, T ; E)$. Essa topologia pode ser tomada como sendo gerada pela convergência forte, fraca ou fraca*.

Definimos a topologia $\Theta_{l o c}^{+}$no espaço $\mathcal{F}_{l o c}^{+}$da seguinte forma: uma sequência $\left\{u_{m}\right\}_{m \in \mathbb{N}} \subset$ $\mathcal{F}_{\text {loc }}^{+}$converge para uma função $u \in \mathcal{F}_{l o c}^{+}$se $\left\{\Pi_{[0, T]} u_{m}\right\}_{m \in \mathbb{N}} \subset \mathcal{F}(0, T ; E)$ converge para $\Pi_{[0, T]} u \in \mathcal{F}(0, T ; E)$ na topologia $\Theta_{[0, T]}$, para todo $T>0$. Assumimos que o espaço $\mathcal{F}_{\text {loc }}^{+}$ munido da topologia $\Theta_{l o c}^{+}$é um espaço de Hausdorff. Se $\Theta_{l o c}^{+}$é metrizável, então essa propriedade é sempre válida.

O conjunto $\mathcal{K}^{+}$munido da topologia induzida $\Theta_{l o c}^{+}$é denominado espaço de trajetórias.

Proposição 2.2.3. O semigrupo $\left\{T(t): \mathcal{F}_{l o c}^{+} \rightarrow \mathcal{F}_{l o c}^{+}\right\}$é contínuo na topologia $\Theta_{l o c}^{+}$.

Demonstração: Se $u_{m} \rightarrow u$ em $\Theta_{+}^{l o c}$, então $\Pi_{[0, T]} u_{m} \rightarrow \Pi_{[0, T]} u$ em $\Theta_{[0, T]}$, para todo $T \geqslant 0$. Em particular, $\Pi_{[0, T+t]} u_{m} \rightarrow \Pi_{[0, T+t]} u$ em $\Theta_{[0, T+t]}$ para todo $t \geqslant 0$. Como $T(t): \mathcal{F}(0, T+t ; E) \rightarrow \mathcal{F}(0, T ; E)$ é uma aplicação contínua, segue que $\Pi_{[0, T]} T(t) u_{m} \rightarrow$ $\Pi_{[0, T]} T(t) u$ em $\Theta_{[0, T]}$, para todo $T \geqslant 0$, isto é, $T(t) u_{m} \rightarrow T(t) u$ em $\Theta_{+}^{l o c}$. Portanto, $T(t)$ é contínuo para todo $t \geqslant 0$.

A continuidade do semigrupo $\left\{T(t): \mathcal{F}_{l o c}^{+} \rightarrow \mathcal{F}_{l o c}^{+}\right\}$na topologia $\Theta_{l o c}^{+}$é uma propriedade importante, pois nos permite utilizar a teoria clássica para a construção de atratores globais de semigrupos contínuos.

Definamos o espaço de Banach

$$
\mathcal{F}_{b}^{+}=\left\{u \in \mathcal{F}_{l o c}^{+}:\|u\|_{\mathcal{F}_{b}^{+}}<+\infty\right\}
$$


onde a norma em $\mathcal{F}_{b}^{+}$é dada por

$$
\|u\|_{\mathcal{F}_{b}^{+}}=\sup _{t \geqslant 0}\left\|\Pi_{[0,1]} T(t) u\right\|_{\mathcal{F}(0,1 ; E)}
$$

O espaço $\left(\mathcal{F}_{b}^{+},\|\cdot\|_{\mathcal{F}_{b}^{+}}\right)$é necessário para definir a classe de conjuntos limitados que serão atraídos pelo atrator sob o ação do semigrupo. Assumimos que $\mathcal{K}^{+} \subset \mathcal{F}_{b}^{+}$, isto é, toda solução fraca global $u$ de $(2.42)$ pertencente a $\mathcal{K}^{+}$satisfaz $\|u\|_{\mathcal{F}_{b}^{+}}<+\infty$. $\Theta_{l o c}^{+}$.

Vamos estabelecer um critério de compacidade no espaço $\mathcal{F}_{l o c}^{+}$munido da topologia

Proposição 2.2.4. Um conjunto $B \subset \mathcal{F}_{\text {loc }}^{+}$é compacto na topologia $\Theta_{\text {loc }}^{+}$se, e somente se, o conjunto $\Pi_{[0, T]} B$ é compacto em $\mathcal{F}(0, T ; E)$ na topologia $\Theta_{[0, T]}$, para todo $T \geqslant 0$.

Demonstração: $(\Rightarrow)$ Se $\left\{u_{m}\right\} \subset B$ é uma sequência arbitrária, então, por hipótese, existe uma subsequência $\left\{u_{m, j}\right\} \subset\left\{u_{m}\right\}$ convergente na topologia $\Theta_{l o c}^{+}$. Logo, $\left\{\Pi_{[0, T]} u_{m, j}\right\}$ é uma subsequência de $\left\{\Pi_{[0, T]} u_{m}\right\}$ e é convergente na topologia $\Theta_{[0, T]}$ para todo $T>0$. Como tomamos uma sequência arbitrária, segue o resultado.

$(\Leftarrow)$ Suponha que $\Pi_{[0, T]} B=\left\{\varphi:[0, T] \rightarrow E:\right.$ existe $u \in B$ tal que $\left.\Pi_{[0, T]} u=\varphi\right\}$ é compacto para todo $T>0$. Consideremos as sequências $\left\{u_{m}\right\} \subset B$ e $\left\{T_{m}\right\} \subset \mathbb{R} \operatorname{com} T_{m} \rightarrow+\infty$. Por hipótese, restrito ao segmento $\left[0, T_{1}\right]$ existe uma subsequência $\left\{u_{m, 1}\right\} \subset\left\{u_{m}\right\}$ que converge em $\Theta_{\left[0, T_{1}\right]}$. Analogamente, $\left\{u_{m, 1}\right\}$ contém uma subsequência $\left\{u_{m, 2}\right\}$ convergente em $\Theta_{\left[0, T_{2}\right]}$, $T_{2}>T_{1}$. Repetindo esse processo, obtemos uma subsequência $\left\{u_{m, k}\right\} \subset\left\{u_{m, k-1}\right\}$ convergente em $\Theta_{\left[0, T_{k}\right]}$ para todo $T_{k}$. Considere agora a subsequência diagonal $\left\{u_{m, m}: m=1,2, \ldots\right\}$ que é uma subsequência de toda sequência $\left\{u_{m, k}\right\}$ a partir de $k \geqslant m$. Temos que $\left\{u_{m, m}\right\}$ converge em na topologia $\Theta_{\left[0, T_{k}\right]}$ para todo $T_{k}$, e, portanto, $\left\{u_{m, m}\right\}$ é convergente em $\Theta_{+}^{\text {loc }}$.

Definição 2.2.5. Um conjunto $P \subset \mathcal{K}^{+}$é um conjunto atrativo para o semigrupo $\{T(t)\}$ na topologia $\Theta_{\text {loc }}^{+}$, se para todo conjunto $B \subset \mathcal{K}^{+}$(limitado na norma de $\mathcal{F}_{b}^{+}$) temos que $T(t) B$ é atraído por $P$ quando $t \rightarrow+\infty$, isto é, para toda vizinhança $\mathcal{O}(P) \supset P$ na topologia $\Theta_{\text {loc }}^{+}$, existe $\tau=\tau(B, \mathcal{O}) \geqslant 0$ tal que $T(t) B \subset \mathcal{O}(P)$, para todo $t \geqslant \tau$.

Definição 2.2.6. Um conjunto $P \subset \mathcal{K}^{+}$é um conjunto absorvente para o semigrupo $\{T(t)\}$ se, para todo conjunto $B \subset \mathcal{K}^{+}$(limitado na norma de $\mathcal{F}_{b}^{+}$), existe $\tau=\tau(B) \geqslant 0$ tal que $T(t) B \subset P$, para todo $t \geqslant \tau$.

Definição 2.2.7. Um conjunto $\mathfrak{A} \subset \mathcal{K}^{+}$é o atrator de trajetórias do semigrupo $\{T(t)\}$ restrito a $\mathcal{K}^{+}$munido da topologia $\Theta_{\text {loc }}^{+}$, quando

(i) $\mathfrak{A}$ é limitado na norma do espaço $\mathcal{F}_{b}^{+}$e compacto na topologia $\Theta_{\text {loc }}^{+}$;

(ii) $\mathfrak{A}$ é um conjunto atrativo na topologia $\Theta_{\text {loc }}^{+}$; 
2 Construção do atrator de trajetórias de equações de evolução abstratas

(ii) $\mathfrak{A}$ é estritamente invariante, isto é, $T(t) \mathfrak{A}=\mathfrak{A}$, para todo $t \geqslant 0$.

Segue da Definição 2.2.7 que se o atrator de trajetórias $\mathfrak{A}$ do semigrupo $\{T(t)\}$ restrito a $\mathcal{K}^{+}$existe, então ele é único.

No próximo resultado estabelecemos a existência do atrator de trajetórias para a equação (2.42).

Teorema 2.2.8. Suponha que o espaço de trajetórias $\mathcal{K}^{+}$da equação (2.42) é tal que $\mathcal{K}^{+} \subset$ $\mathcal{F}_{b}^{+}$e satisfaz a propriedade (2.43). Se existe um conjunto atrativo $P \subset \mathcal{K}^{+}$com respeito ao semigrupo $\{T(t)\}$ limitado na norma de $\mathcal{F}_{+}^{b}$ e compacto na topologia $\Theta_{\text {loc }}^{+}$, então o semigrupo $\{T(t)\}$ tem um atrator de trajetórias $\mathfrak{A} \subset P$. Além disso, o conjunto $\mathfrak{A}$ é compacto em $\Theta_{\text {loc }}^{+}$ e limitado em $\mathcal{F}_{b}^{+}$.

Demonstração: Pela propriedade (2.43), o espaço de trajetórias $\mathcal{K}^{+}$é invariante com respeito ao semigrupo $\{T(t)\}$. Logo, podemos considerar $T(t): \mathcal{K}^{+} \rightarrow \mathcal{K}^{+}$. Sabemos, pela Proposição 2.2.3, que essa aplicação é contínua e, por hipótese, $P \subset \mathcal{K}^{+}$é um conjunto atrativo para $T(t)$, compacto na topologia $\Theta_{l o c}^{+}$e limitado na norma do espaço $\mathcal{F}_{b}^{+}$. Assim, estamos nas condições do Teorema 1.4.4, donde existe um $\left(\mathcal{F}_{b}^{+}, \mathcal{F}_{\text {loc }}^{+}\right)$-atrator $\mathfrak{A} \subset P$ que é o atrator de trajetórias que procuramos.

Observação 2.2.1. Se existe um conjunto absorvente $P$ para o semigrupo $\{T(t)\}$ tal que $P$ é limitado na norma de $\mathcal{F}_{b}^{+}$e compacto na topologia $\Theta_{\text {loc }}^{+}$e, além disso, a topologia $\Theta_{\text {loc }}^{+}$é metrizável sobre o conjunto $P$, então o Teorema 2.2.8 se reduz ao Teorema 1.3.10 que trata da existência de um atrator global para o semigrupo contínuo $\{T(t)\}$ restrito ao espaço métrico P. Nesse caso, o atrator de trajetórias é dado pela fórmula

$$
\mathfrak{A}=\cap_{t \geqslant 0} T(t) P .
$$

Vamos agora obter uma descrição do atrator de trajetórias $\mathfrak{A}$ da equação (2.42) em termos do núcleo dessa equação.

Consideremos a equação

$$
\partial_{t} u=A(u), t \in \mathbb{R}
$$

Introduzimos anteriormente o espaço de trajetórias $\mathcal{K}^{+}$da equação $(2.45)$, cujos elementos são soluções fracas globais de (2.45) definidas sobre o semi-eixo $\mathbb{R}_{+}$. Vamos extender essa definição para todo o eixo real.

Analogamente ao espaço $\mathcal{F}(0, T ; E)$, assumimos que $\mathcal{F}(-T, T ; E)=$ $\{u:[-T, T] \rightarrow E\}$ é um espaço de Banach. Vamos considerar as soluções fracas da equação 
(2.45) em $\mathcal{F}(-T, T ; E)$ que, por definição, satisfazem a igualdade (2.45) no sentido de distribuição do espaço $\mathcal{D}^{\prime}(-T, T ; E)$, onde $\mathcal{F}(-T, T ; E) \subset \mathcal{D}^{\prime}(-T, T ; E)$.

Definimos

$$
\mathcal{F}_{\text {loc }}=\left\{u: \mathbb{R} \rightarrow E: \Pi_{[-T, T]} T(t) u \in \mathcal{F}(-T, T ; E), \text { para todo } T>0\right\}
$$

e o espaço de Banach

$$
\mathcal{F}_{b}=\left\{u \in \mathcal{F}^{l o c}:\|u\|_{\mathcal{F}_{b}}<+\infty\right\}
$$

onde a norma em $\mathcal{F}_{b}$ é dada pela fórmula

$$
\|u\|_{\mathcal{F}_{b}}=\sup _{t \in \mathbb{R}}\left\|\Pi_{[0,1]} T(t) u\right\|_{\mathcal{F}(0,1 ; E)}
$$

Consideramos o espaço $\mathcal{F}_{l o c}$ munido da topologia $\Theta_{\text {loc }}$ que, em termos de sequências, é dada por: $u_{n} \rightarrow u$ se $\Pi_{[-T, T]} T(t) u_{n} \rightarrow \Pi_{[-T, T]} T(t) u$ em $\Theta_{[-T, T]}$ para todo $T>0$.

Observemos que a família de operadores $T(t): \mathcal{F}_{l o c} \rightarrow \mathcal{F}_{l o c}$ dados por

$$
T(t) u(s)=u(t+s)
$$

para todo $t \in \mathbb{R}$ e $s \in \mathbb{R}$, forma um grupo sobre $\mathcal{F}_{\text {loc }}$.

Dizemos que uma função $u: \mathbb{R} \rightarrow E$ é uma solução completa da equação (2.45) se $u \in \mathcal{F}_{l o c}$ e a função $\prod_{[-T, T]} u$ satisfaz $(2.45)$ no sentido de distribuição do espaço $\mathcal{D}^{\prime}(-T, T ; E)$, para todo $T>0$.

Definição 2.2.9. O núcleo $\mathcal{K}$ da equação (2.45) é o conjunto de todas as trajetórias completas $u$ de (2.45) tais que $\Pi_{+} T(t) u \in \mathcal{K}^{+}$e que são limitadas na norma de $\mathcal{F}_{b}$, isto é, existe uma constante $C_{u}>0$ tal que

$$
\left\|\Pi_{[0,1]} T(t) u\right\|_{\mathcal{F}(0,1 ; E)} \leqslant C_{u}
$$

para todo $t \in \mathbb{R}$.

Teorema 2.2.10. Se as hipóteses do Teorema 2.2.8 estão satisfeitas, então

$$
\mathfrak{A}=\Pi_{+} \mathcal{K} .
$$

Além disso, o conjunto $\mathcal{K}$ é compacto na topologia $\Theta_{\text {loc }}$ e limitado na norma do espaço $\mathcal{F}_{b}$.

Demonstração: Se $u \in \mathcal{K}$, então $\Pi_{+} T(t) u \in \mathcal{K}^{+}$, para todo $t \in \mathbb{R}$. Consideremos o conjunto $B=\left\{\Pi_{+} T(t) u: t \in \mathbb{R}\right\} \subset \mathcal{F}_{b}^{+}$. Temos que $B \subset \mathcal{K}^{+}$e é estritamente invariante pelo semigrupo 
$\{T(t)\}$. Como $\mathfrak{A}$ atrai $T(t) B=B$ quando $t \rightarrow+\infty$, então $B$ está contido em toda vizinhança de $\mathfrak{A}$. Além disso, $\mathfrak{A}$ é um conjunto compacto no espaço de Hausdorff $\mathcal{F}_{\text {loc }}^{+}$, donde $B \subset \mathfrak{A}$, isto é, $\Pi_{+} \mathcal{K} \subseteq \mathfrak{A}$.

Reciprocamente, seja $u_{0} \in \mathfrak{A}$. Pela invariância de $\mathfrak{A}$, existe uma função $u \in \mathcal{K}$ tal que $\Pi_{+} T(t) u \in \mathfrak{A}$, para todo $t \in \mathbb{R}$, e $\Pi_{+} u=u_{0}$. Assim, $\mathfrak{A} \subseteq \Pi_{+} \mathcal{K}$.

O conjunto $\mathcal{K}$ é compacto em $\Theta_{\text {loc }}$, pois $\Pi_{+} \mathcal{K}$ é compacto em $\Theta_{\text {loc }}^{+}$.

Corolário 2.2.11. Suponha que as hipóteses do Teorema 2.2.8 estão satisfeitas. Se $B \subset \mathcal{K}^{+}\left(\subset \mathcal{F}_{\text {loc }}^{+}\right)$, então o conjunto $\Pi_{[0, T]} T(t) B$ está contido numa vizinhança de $\Pi_{[0, T]} \mathcal{K}=$ $\Pi_{[0, T]} \mathfrak{A}$ na topologia $\Theta_{[0, T]}$ quando $t \rightarrow \infty$, para todo $T>0$. Em particular, se $\Theta(0, T ; E)$ é metrizável então

$$
\operatorname{dist}_{\Theta(0, T ; E)}\left(\Pi_{[0, T]} T(t) B, \Pi_{[0, T]} \mathcal{K}\right) \rightarrow 0
$$

quando $t \rightarrow \infty$.

Observação 2.2.2. Exemplos de equações onde se aplica o roteiro acima para a construção do atrator de trajétorias podem ser encontrados nos trabalhos de Chepyzhov, V. V. and Vishik, M. I. [9], [11], [12], [13], [14], [15], [16].

\subsubsection{Atrator global fraco}

Nesta subseção vamos definir o atrator global fraco para a equação (2.42) e o relacionaremos com o atrator de trajetórias $\mathfrak{A}$.

Sejam $E$ e $E_{0}$ espaços de Banach tais que $E \hookrightarrow E_{0}$. Assumimos que toda trajetória $u$ da equação $(2.42)$ satisfaz $u \in L^{\infty}\left(\mathbb{R}_{+}, E\right)$ e é uma função fracamente contínua com valores num espaço de Banach $E_{0}$, então, pelo Teorema 1.1.18, $u$ é uma função fracamente contínua com valores em $E$, isto é, para todo funcional $\varphi \in E^{\prime}$ a função $\langle u, \varphi\rangle$ é contínua sobre $\mathbb{R}_{+}$. Se $u \in \mathcal{K}^{+}$, então $u(s) \in E \mathrm{e}$

$$
\|u(s)\|_{E} \leqslant\|u\|_{L^{\infty}\left(\mathbb{R}_{+}, E\right)}
$$

para todo $s \geqslant 0$.

Suponhamos que $\mathcal{F}_{b}^{+} \subset L^{\infty}\left(\mathbb{R}_{+} ; E\right)$ e a topologia $\Theta_{\text {loc }}^{+}$é mais forte (ou mais fina) do que a topologia do espaço $C_{w}\left(\mathbb{R}_{+} ; E\right)=\left\{u: \mathbb{R}_{+} \rightarrow E: u\right.$ é fracamente contínua $\}$. Logo, se uma sequência de funções $\left\{u_{m}\right\} \subset \mathcal{F}_{\text {loc }}^{+}$é convergente na topologia $\Theta_{l o c}^{+}$, então essa sequência também converge na topologia do espaço $C_{w}\left(\mathbb{R}_{+} ; E\right)$. Uma referência que trata de comparação entre topologias é o livro de Munkres, J. R. [38], página 77.

Seja $\mathcal{B} \subset \mathcal{K}^{+}$um subconjunto arbitrário de trajetórias. Denotamos a seção do 
conjunto $\mathcal{B}$ no instante $t$ por

$$
\mathcal{B}(t)=\{u(t): u \in \mathcal{B}\} \subset E .
$$

Os conjuntos $\mathcal{B}(t)$ são uniformemente limitados em $E$, isto é,

$$
\|\mathcal{B}(t)\|_{E} \doteq \sup _{u \in \mathcal{B}(t)}\|u\|_{E} \leqslant\|\mathcal{B}\|_{L^{\infty}\left(\mathbb{R}_{+} ; E\right)}
$$

Se $\mathfrak{A} \subset \mathcal{K}^{+}$é o atrator de trajetórias da equação (2.42), então o conjunto $\mathfrak{A}$ é limitado em $L^{\infty}\left(\mathbb{R}_{+} ; E\right)$, pois $\mathcal{F}_{b}^{+} \subset L^{\infty}\left(\mathbb{R}_{+} ; E\right)$ e é compacto em $C_{w}\left(\mathbb{R}_{+} ; E\right)$, pois a topologia $\Theta_{l o c}^{+}$é mais fina do que a topologia do espaço $C_{w}\left(\mathbb{R}_{+} ; E\right)$.

Consideremos a seção de $\mathfrak{A}$ no instante $t$, isto é,

$$
\mathfrak{A}(t)=\{u(t): u \in \mathfrak{A}\} \subset E .
$$

A propriedade de invariância estrita do atrator de trajetórias $\mathfrak{A}$ com relação ao semigrupo $\{T(t)\}$ implica que

$$
\mathfrak{A}(t)=\mathfrak{A}(0),
$$

para todo $t \geqslant 0$. Além disso, como $\mathfrak{A}$ é limitado em $L^{\infty}\left(\mathbb{R}_{+} ; E\right)$ e compacto em $C_{w}\left(\mathbb{R}_{+} ; E\right)$, segue que o conjunto $\mathfrak{A}(0)(=\mathcal{K}(0))$ é limitado em $E$, fracamente fechado em $E$ e, portanto, fortemente fechado em $E$. Observemos que, pelo Teorema 2.2.10, temos $\mathfrak{A}(0)=\mathcal{K}(0)$, onde $\mathcal{K}(0)$ é a seção do núcleo $\mathcal{K}$ da equação $(2.42)$ no espaço $\mathcal{F}_{b}$.

Definição 2.2.12. Um conjunto $\mathcal{A} \subset E$ é o atrator global fraco da equação (2.42) se é um subconjunto fechado e limitado e satisfaz as seguintes propriedades

(1) para todo conjunto $\mathcal{B} \subset \mathcal{K}^{+} \subset \mathcal{F}_{b}^{+}$, a seção $\mathcal{B}(t)$ é atraída por $\mathcal{A}$ na topologia fraca de $E$ quando $t \rightarrow+\infty$, isto é, para toda vizinhança $\mathcal{O}_{w}(\mathcal{A}) \supset \mathcal{A}$ na topologia fraca de $E$ existe $\tau=\tau\left(\mathcal{B}, \mathcal{O}_{w}\right)$ tal que

$$
\mathcal{B}(t) \subset \mathcal{O}_{w}(\mathcal{A})
$$

para todo $t \geqslant \tau$;

(2) $\mathcal{A} \subset E$ é um conjunto limitado e fechado minimal que atrai as seções de todos os elementos de $\mathcal{K}^{+}$na topologia fraca de $E$ quando $t \rightarrow+\infty$.

Teorema 2.2.13. Suponhamos que as hipóteses do Teorema 2.2.8 estão satisfeitas. Se $\mathcal{F}_{b}^{+} \subset L^{\infty}\left(\mathbb{R}_{+} ; E\right)$ e a topologia $\Theta_{\text {loc }}^{+}$é mais fina do que a topologia do espaço $C_{w}\left(\mathbb{R}_{+} ; E\right)$, então o conjunto $\mathcal{A} \doteq \mathfrak{A}(0)=\mathcal{K}(0)$ é o atrator global fraco da equação (2.42) 
Demonstração: Ver Chepyzhov, V. V. and Vishik, M. I. [9], Teorema 3.1, Página 225.

Observação 2.2.3. Se na Definição 2.2.12 o conjunto $\mathcal{A}$ é compacto em $E$ e atrai as seções $\mathcal{B}(t)$ na norma de $E$, então dizemos que $\mathcal{A}$ é o atrator global forte de (2.42).

Para finalizar essa subseção, vamos construir o atrator global do semigrupo solução associado ao problema de Cauchy para a equação (2.42) usando o atrator de trajetórias.

Assumimos que o espaço de trajetórias $\mathcal{K}^{+}$da equação (2.42) satisfaz a seguinte propriedade: para todo $u_{0} \in E$ existe uma única trajetória $u \in \mathcal{K}^{+}$tal que

$$
u(0)=u_{0}
$$

e $u(t) \in E$, para todo $t \geqslant 0$ e para toda trajetória $u \in \mathcal{K}^{+}$. Assim, podemos definir a família de operadores $\{S(t)\}$ associada ao problema de Cauchy para a equação (2.42), a saber

$$
S(t): E \rightarrow E, \quad S(t) u_{0}=u(t)
$$

A unicidade de solução do problema de Cauchy implica que a família $\{S(t): t \geqslant 0\}$ é um semigupo sobre $E$.

O próximo resultado estabelece a existência do atrator global para o semigrupo $\{S(t)\}$.

Teorema 2.2.14. Suponhamos que as hipóteses do Teorema 2.2.8 estão satisfeitas. Assumimos que o semigrupo translação $\{T(t)\}$ tem um conjunto atrativo $P_{1} \subset \mathcal{K}^{+}$, compacto em $C\left(\mathbb{R}_{+}, E\right)$ e o problema (2.42), (2.49) tem solução única em $\mathcal{K}^{+}$. Além disso, $\mathcal{F}_{b}^{+} \subset C_{b}\left(\mathbb{R}_{+} ; E\right)$ e para todo subconjunto limitado $B_{0} \subset E$, as trajetórias $u \in \mathcal{K}^{+}$tais que $u(0) \in B_{0}$ pertencem a um conjunto limitado $\mathcal{B}=\mathcal{B}\left(B_{0}\right) \subset \mathcal{F}_{b}^{+}$. Então o semigrupo $\{S(t)\}$ tem um atrator global $\mathcal{A} \subset E$, que é compacto $e$

$$
\mathcal{A}=\mathfrak{A}(0)=\{u(0): u \in \mathfrak{A}\}
$$

Demonstração: Ver Chepyzhov, V. V. and Vishik, M. I. [9], Teorema 3.2, Página 227. 


\section{Atrator de trajetórias de uma equação parabólica}

Há muitos trabalhos que lidam com sistemas de equações de reação-difusão da forma

$$
\left\{\begin{array}{l}
\frac{\partial u}{\partial t}=D \Delta u+f(u) \quad(t, x) \in(0, \infty) \times \Omega \\
\frac{\partial u}{\partial \nu}=0 \quad(t, x) \in(0, \infty) \times \Gamma
\end{array}\right.
$$

onde $\Omega \subset \mathbb{R}^{n}$ é um subconjunto aberto, limitado, conexo, com fronteira suave $\Gamma=\partial \Omega$, $u \in \mathbb{R}^{m}, D=\operatorname{diag}\left(d_{1}, \ldots, d_{m}\right), d_{j}>0$ é uma constante e $f: \mathbb{R}^{m} \rightarrow \mathbb{R}^{m}$ é uma função de classe $C^{2}$; dentre eles, destacamos os que tratam da caracterização do fluxo de (3.1) em termos da equação diferencial ordinária

$$
\frac{d u}{d t}=f(u)
$$

Esse problema foi estudado por muitos autores, dos quais mencionamos Carvalho, A. N. [5], [6], Hale, J. K. [22], Hale, J. K. Hale and Rocha, C. [24], [25] e Conway, E., Hoff, D. and Smoller, J. [19] e observou-se que quando o coeficiente de difusão é grande existe perda da dependência espacial no comportamento assintótico de suas soluções. Isso porque há um "gap" entre o autovalor zero do operador laplaciano com condição de Neumann e seu primeiro autovalor positivo, o que assegura que o espaço das funções constantes é uma variedade invariante exponencialmente atratora.

Em particular, em Conway, E.; Hoff, D.; Smoller, J. [19] o teorema principal afirma que existem constantes $c_{1}, c_{2}>0$ tais que para cada $t>0$ temos

$$
\|\nabla u(t, \cdot)\|_{L^{2}} \leqslant c_{1} e^{-\sigma t} \text { e }\|u(t, \cdot)-\bar{u}(t)\|_{L^{2}} \leqslant c_{2} e^{-\sigma t}
$$


3 Atrator de trajetórias de uma equação parabólica

onde $u \in \mathbb{R}^{m}, m \geqslant 1$, é a solução do seguinte sistema de equações de reação-difusão

$$
\left\{\begin{array}{l}
\frac{\partial u}{\partial t}=D \Delta u+\sum_{j=1}^{n} A_{j}(x, u) \frac{\partial u}{\partial x_{j}}+f(u) \quad(t, x) \in(0, \infty) \times \Omega \\
\frac{\partial u}{\partial \nu}=0 \quad(t, x) \in(0, \infty) \times \Gamma \\
u(0, x)=u_{0}(x) \quad x \in \Omega,
\end{array}\right.
$$

$\Omega \subset \mathbb{R}^{n}, n \geqslant 1$, é um subconjunto aberto, limitado, conexo, com fronteira suave $\Gamma, D>0$ é uma matriz diagonal cujas entradas são constantes positivas, $A_{j}$ 's são funções matriciais contínuas, $\bar{u}$ é a média de $u$ em $\Omega$ e é a solução de uma equação diferencial ordinária, e $\sigma=d \lambda-M-a \sqrt{n \lambda}$ onde $\lambda$ é o menor autovalor positivo do operador $-\Delta$ em $\Omega$ com condição de fronteira Neumann homogênea, $a \doteq\left\{\left|A_{j}(x, u)\right|: x \in \bar{\Omega}, u \in \Sigma, 1 \leqslant j \leqslant n\right\}$ com $\Sigma=\bigcap_{k=1}^{m}\left\{u \in \mathbb{R}^{m}: a_{k} \leqslant u_{k} \leqslant b_{k}\right\}$, onde $-\infty<a_{k}<b_{k}<\infty$, d denota o menor autovalor da matriz $D$ e $M \doteq \max \left\{\left|f^{\prime}(u)\right|: u \in \Sigma\right\}$. Observemos que se $d$ é grande, então $\sigma$ é grande. Assim, para cada $t>0$ fixo o gradiente de $u$ fica pequeno e $u$ tende para sua média quando o parâmetro de difusão torna-se grande.

Um resultado sem a hipótese restritiva de existência de uma região invariante $\Sigma$ para (3.1) foi obtido por Hale, J. [23] e o transcrevemos a seguir

Teorema 3.0.15. Sejam $\Omega \subset \mathbb{R}^{n}, X=L^{2}\left(\Omega, \mathbb{R}^{m}\right), A=-\Delta: \operatorname{dom}(A) \rightarrow X, \operatorname{dom}(A)=$ $\left\{\phi \in W^{2,2}\left(\Omega, \mathbb{R}^{m}\right): \frac{\partial \phi}{\partial \nu}=0\right.$ em $\left.\Gamma\right\}$ e consideremos $\alpha>\frac{3}{4}$ e $n \leqslant 3$. Se $\mathcal{A}$ é um atrator compacto da equação (3.2), então existe $\delta>0$ tal que $\mathcal{A}$ considerado como subconjunto do espaço das funções constantes de $X^{\alpha}$ é um atrator compacto da equação (3.1) se $d \lambda>\delta$. Ou seja, existe uma vizinhança $V \supset \mathcal{A}$ em $X^{\alpha}$ e constantes $K>0, c>0$ tais que para todo $u_{0} \in V$, a solução $u$ da equação (3.1) passando por $u_{0}$ em $t=0$ satisfaz

$$
\|u(t, x)-\bar{u}(t)\|_{X^{\alpha}} \leqslant K e^{-c t}, \quad t \geqslant 0
$$

onde $u(t)=\frac{1}{|\Omega|} \int_{\Omega} u(t, x) d x$ e $\bar{u}(t)$ satisfaz a equação

$$
\frac{d \bar{u}(t)}{d t}=f(\bar{u}(t))+g\left(t, u_{0}\right)
$$

onde $\left|g\left(t, u_{0}\right)\right| \leqslant K e^{-c t}, t \geqslant 0$.

Observemos que os trabalhos citados acima lidam com problemas bem postos. No contexto de problemas de Cauchy sem unicidade de solução e envolvendo o objeto de estudo deste trabalho, o atrator de trajetórias, não encontramos referências na literatura. Pretendemos então dar a nossa contribuição ao estudo do comportamento assintótico de problemas parabólicos com difusão grande, cujo problema de Cauchy correspondente não goza da propriedade de unicidade. Nossa pesquisa vai se restringir à investigação do comportamento 
assintótico das soluções do seguinte problema de reação-difusão quando fazemos $d \rightarrow+\infty$

$$
\left\{\begin{array}{l}
\frac{\partial u}{\partial t}=d \Delta u-f(u)+|u|^{\alpha-1} u \quad(t, x) \in(0, \infty) \times \Omega \\
\frac{\partial u}{\partial \nu}=0 \quad(t, x) \in(0, \infty) \times \Gamma
\end{array}\right.
$$

onde $\Omega \subset \mathbb{R}^{n}(n \geqslant 3)$ é um subconjunto aberto, limitado, conexo, com fronteira suave $\Gamma \mathrm{e}$ para cada ponto $x \in \Gamma, \nu=\nu(x)$ indica o vetor normal exterior unitário a $\Gamma$ no ponto $x$. Além disso, $d>0, \alpha \in(0,1)$ e $f: \mathbb{R} \rightarrow \mathbb{R}$ é uma função de classe $C^{1}$ satisfazendo as seguintes hipóteses: existem constantes positivas $c_{1}, c_{2}, c_{3}, C, c_{f}$ e $2<p<\frac{2 n}{n-2}$ tais que

$$
\begin{gathered}
f^{\prime}(u) \geqslant c_{f}, \\
c_{1}|u|^{p}-c_{3} \leqslant f(u) \cdot u \\
|f(u)|^{\frac{p}{p-1}} \leqslant c_{2}\left(|u|^{p}+1\right)
\end{gathered}
$$

e

$$
\left|f\left(u_{1}\right)-f\left(u_{2}\right)\right| \leqslant C|u-v|\left(1+\left|u_{1}\right|^{p-2}+\left|u_{2}\right|^{p-2}\right),
$$

para quaisquer $u, u_{1}, u_{2} \in \mathbb{R}$. Destacamos que a desigualdade (3.5) é denominada condição de dissipatividade de $f$.

Vamos começar aplicando o roteiro da Seção 2.2 na construção do atrator de trajetórias $\mathfrak{A}_{d}$ de (3.3) para cada $d>0$. Isso será feito na Seção 3.1 sem a hipótese (3.7) que é desnecessária nesse ponto.

Além disso, na Seção 3.2 vamos considerar a equação (3.3) com um parâmetro $\epsilon$ pequeno no seu termo de reação e construiremos o correspondente atrator de trajetórias. Faremos isso para o problema a seguir

$$
\left\{\begin{array}{l}
\frac{\partial u}{\partial t}=d \Delta u-f(u)+\epsilon|u|^{\alpha-1} u \quad(t, x) \in(0, \infty) \times \Omega \\
\frac{\partial u}{\partial \nu}=0 \quad(t, x) \in(0, \infty) \times \Gamma,
\end{array}\right.
$$

com as hipóteses (3.4)-(3.6) e verificaremos que o atrator de trajetórias do problema envolvendo o parâmetro $\epsilon$ está contido numa vizinhança do atrator de trajetórias do respectivo problema limite com relação a topologia $\Theta_{l o c}^{+}$quando $\epsilon \rightarrow 0^{+}$. Este será o conteúdo da Seção 3.2 .

E, finalmente, na Seção 3.3 vamos adotar o sistema ortonormal completo para $L^{2}(\Omega)$ 
dado pelas autofunções $\left\{w_{j}\right\}_{j=0}^{\infty}$ do problema de Neumann

$$
\left\{\begin{array}{l}
\Delta w+\lambda w=0 \quad \text { em } \Omega \\
\left.\frac{\partial w}{\partial \nu}\right|_{\Gamma}=0
\end{array}\right.
$$

e indicaremos por $Y$ o espaço das funções ortogonais a autofunção $w_{0}$ associada ao autovalor $\lambda_{0}=0$. Por meio da decomposição do espaço $L^{2}(\Omega)=\left[w_{0}\right] \oplus Y$ vamos reescrever a equação (3.3) como um sistema de equações. Considerando a equação diferencial ordinária

$$
\left\{\begin{array}{l}
\dot{\gamma}(t)=-f(\gamma(t))+|\gamma(t)|^{\alpha-1} \gamma(t) \\
\gamma(0)=\gamma_{0} \in \mathbb{R}
\end{array}\right.
$$

e o seu respectivo atrator de trajetórias $\mathfrak{A}_{\infty}$, demonstraremos que a família $\left\{\mathfrak{A}_{d}\right\}_{d} \cup \widetilde{\mathfrak{A}_{\infty}}$ é semicontínua superiormente em $d=+\infty$, onde $\widetilde{\mathfrak{A}_{\infty}}$ é a imersão de $\mathfrak{A}_{\infty}$ em $\left[w_{0}\right] \oplus Y$.

\subsection{Construção do atrator de trajetórias de um pro- blema parabólico}

Seja $\Omega$ um subconjunto aberto, limitado, conexo do $\mathbb{R}^{n}(n \geqslant 3)$ e com fronteira suave $\Gamma$. Para cada ponto $x \in \Gamma$, indicaremos por $\nu=\nu(x)$ o vetor normal exterior unitário a $\Gamma$ no ponto $x$. Consideremos o seguinte problema de reação-difusão com condição de Neumann

$$
\left\{\begin{array}{l}
\frac{\partial u}{\partial t}=d \Delta u-f(u)+|u|^{\alpha-1} u \quad(t, x) \in(0, \infty) \times \Omega \\
\frac{\partial u}{\partial \nu}=0 \quad(t, x) \in(0, \infty) \times \Gamma
\end{array}\right.
$$

$d>0$ está fixado, $\alpha \in(0,1)$ e $f: \mathbb{R} \rightarrow \mathbb{R}$ é uma função de classe $C^{1}$ satisfazendo as seguintes hipóteses: existem constantes positivas $c_{1}, c_{2}, c_{3}$ e $2<p<\frac{2 n}{n-2}$ tais que

$$
\begin{gathered}
f^{\prime}(u) \geqslant c_{f}, \\
c_{1}|u|^{p}-c_{3} \leqslant f(u) \cdot u
\end{gathered}
$$

$\mathrm{e}$

$$
|f(u)|^{\frac{p}{p-1}} \leqslant c_{2}\left(|u|^{p}+1\right)
$$

para todo $u \in \mathbb{R}$.

Vamos identificar os espaços em que trabalharemos e definiremos quando uma função $u=u(t, x)$ será solução da equação (3.10). Observemos que a função $u \mapsto|u|^{\alpha-1} u$ não é 
Lipshchitz e portanto um resultado de unicidade de solução para (3.10) não está assegurado. Assim, a abordagem clássica para estudar a existência de atrator global não se aplica diretamente ao nosso problema e por isso empregaremos a teoria do atrator de trajetórias.

Destacamos que os trabalhos dedicados à construção do atrator de trajetórias de equações de evolução, cujo problema de Cauchy correspondente não tem unicidade, consideram quase sempre a condição de Dirichlet. Não encontramos referências para a condição de Neumann, embora as contas sejam parecidas. Algumas referências no caso Dirichlet são Yue, G. and Zhong, C. [51], Chepyzhov, V. V. and Vishik, M. I. [9], [10], [11], [12], [13], [15], [16] e Chepyzhov, V. V., Vishik, M. I. and Zelik, S. V. [14].

Façamos algumas observações antes de apresentarmos o conceito de solução do nosso problema.

Vamos considerar em $H^{1}(\Omega)$ a norma

$$
\|u\|^{2}=\int_{\Omega}\left(|u|^{2}+d|\nabla u|^{2}\right) d x
$$

para $u \in H^{1}(\Omega)$, que é equivalente a norma usual

$$
\|u\|_{H^{1}(\Omega)}^{2}=\|u\|_{L^{2}(\Omega)}^{2}+\|\nabla u\|_{L^{2}(\Omega)}^{2}
$$

pois para cada $d>0$

$$
\min \{1, d\}\|u\|_{H^{1}(\Omega)}^{2} \leqslant\|u\|_{H_{d}^{1}(\Omega)}^{2} \leqslant \max \{1, d\}\|u\|_{H^{1}(\Omega)}^{2} .
$$

O $H^{1}(\Omega)$ munido da norma (3.14) será denotado por $H_{d}^{1}(\Omega)$.

O espaço dual de $H_{d}^{1}(\Omega)$ será denotado por $H_{d}^{-1}(\Omega)$ e consideraremos o operador contínuo

$$
\begin{aligned}
\Delta: H_{d}^{1}(\Omega) & \longrightarrow \\
u & \longmapsto\langle\varphi, \Delta u\rangle=-\int_{\Omega}^{-1} \nabla u \nabla \varphi d x
\end{aligned}
$$

Observemos que

$$
\|\Delta u\|_{H_{d}^{-1}(\Omega)}=\sup _{\substack{\varphi \in H_{d}^{1}(\Omega) \\\|\varphi\|_{H_{d}^{1}(\Omega)}=1}}|\langle\varphi, \Delta u\rangle| \leqslant \frac{1}{\sqrt{d}}\left(\int_{\Omega}|\nabla u|^{2} d x\right)^{\frac{1}{2}} .
$$

Sejam $T>0$ e $q$ o expoente conjugado de $p$, isto é, $\frac{1}{p}+\frac{1}{q}=1$. Como $p>2$, segue que $1<q<2$. Se $u \in L^{p}\left(0, T ; L^{p}(\Omega)\right)$, então de $(3.13)$ temos $f(u) \in L^{q}\left(0, T ; L^{q}(\Omega)\right)$ e

$$
\|f(u)\|_{L^{q}\left(0, T ; L^{q}(\Omega)\right)}^{q} \leqslant c_{2}\left(\|u\|_{L^{p}\left(0, T ; L^{p}(\Omega)\right)}^{p}+|\Omega| T\right) .
$$


Se $u \in L^{2}\left(0, T ; H_{d}^{1}(\Omega)\right)$, então da continuidade do operador $\Delta$ temos que $\Delta u \in$ $L^{2}\left(0, T ; H_{d}^{-1}(\Omega)\right)$ e observemos que o termo $|u|^{\alpha-1} u \in L^{q}\left(0, T ; L^{q}(\Omega)\right)$, pois

$$
\begin{aligned}
\left\||u|^{\alpha-1} u\right\|_{L^{q\left(0, T ; L^{q}(\Omega)\right)}}^{q} & =\left.\left.\int_{0}^{T} \int_{\Omega}|| u\right|^{\alpha-1} u\right|^{q} d x d t=\int_{0}^{T} \int_{\Omega}|u|^{\alpha q} d x d t \\
& \stackrel{\text { Hölder }}{\leqslant} \int_{0}^{T}\left(\int_{\Omega}|u|^{2} d x\right)^{\frac{\alpha q}{2}}|\Omega|^{1-\frac{\alpha q}{2}} d t \\
& \stackrel{\text { Young }}{\leqslant} \int_{0}^{T}\left[\frac{\alpha q}{2} \int_{\Omega}|u|^{2} d x+\left(1-\frac{\alpha q}{2}\right)|\Omega|\right] d t \\
& \leqslant \frac{\alpha q}{2} \int_{0}^{T} \int_{\Omega}|u|^{2} d x+\frac{2-\alpha q}{2}|\Omega| T<+\infty .
\end{aligned}
$$

Logo, de (3.10) temos que $\partial_{t} u \in L^{2}\left(0, T ; H_{d}^{-1}(\Omega)\right)+L^{q}\left(0, T ; L^{q}(\Omega)\right)$.

Pelo Teorema de imersão de Sobolev 1.1.1 e por dualidade obtemos

$$
\partial_{t} u \in L^{2}\left(0, T ; H^{-1}(\Omega)\right)+L^{q}\left(0, T ; L^{q}(\Omega)\right) \hookrightarrow L^{q}\left(0, T ; H^{-r}(\Omega)\right)
$$

onde $r=\max \left\{1, n\left(\frac{1}{q}-\frac{1}{2}\right)\right\}$.

Assim, se $u \in L^{2}\left(0, T ; H_{d}^{1}(\Omega)\right) \cap L^{p}\left(0, T ; L^{p}(\Omega)\right)$, então a equação (3.10) pode ser considerada no sentido de distribuição do espaço $\mathcal{D}^{\prime}\left(0, T ; H^{-r}(\Omega)\right)$ e $\partial_{t} u \in L^{q}\left(0, T ; H^{-r}(\Omega)\right)$.

Definição 3.1.1. Uma função $u=u(t, x), t \geqslant 0, x \in \Omega$, é uma solução fraca global de (3.10) se $u \in L^{2}\left(0, T ; H_{d}^{1}(\Omega)\right) \cap L^{p}\left(0, T ; L^{p}(\Omega)\right)$ para todo $T>0$ e u satisfaz a equação (3.10) no sentido de distribuição do espaço $\mathcal{D}^{\prime}\left(0, T ; H^{-r}(\Omega)\right)$, isto é, para toda função $\varphi \in$ $L^{p}(\Omega) \cap H_{d}^{1}(\Omega)$ a seguinte igualdade é satisfeita

$$
\frac{d}{d t} \int_{\Omega} u(t, x) \cdot \varphi(x) d x+\int_{\Omega}\left\{d \nabla u(t, x) \cdot \nabla \varphi+\left[f(u(t, x))-|u(t, x)|^{\alpha-1} u(t, x)\right] \cdot \varphi(x)\right\} d x=0 .
$$

Observação 3.1.1. Se u é uma solução fraca de (3.10), então pelo Lema 1.1.13 $u \in C\left([0, T] ; H^{-r}(\Omega)\right)$. Se além disso $u \in L^{\infty}\left(0, T ; L^{2}(\Omega)\right)$, então, pelo Teorema 1.1.18, temos $u \in C_{w}\left([0, T] ; L^{2}(\Omega)\right)$. Logo, (3.10) está bem definido quando $u_{0} \in L^{2}(\Omega)$.

Teorema 3.1.2. Se $f$ satisfaz (3.12)-(3.13) e $u_{0} \in L^{2}(\Omega)$, então existe uma solução fraca global $u$ de (3.10), satisfazendo $u \in L^{p}\left(0, T ; L^{p}(\Omega)\right) \cap L^{2}\left(0, T ; H_{d}^{1}(\Omega)\right) \cap L^{\infty}\left(0, T ; L^{2}(\Omega)\right) e$ $u(0)=u_{0}$ para todo $T>0$.

Demonstração: Vamos aplicar o método de Faedo-Galerkin que consiste das seguintes etapas

(i) aproximações de Galerkin, que consiste em projetar o problema em subespaços de dimensão finita obtendo um problema aproximado. Este por sua vez é um sistema de 
equações diferencias ordinárias com dados iniciais, cuja existência de solução local é garantida pelo Teorema de Carathéodory 1.1.21;

(ii) estimativas a priori, onde procuramos limitantes para a solução aproximada e sua derivada. E utilizando o Teorema de prolongamento de soluções 1.1.23 podemos estender a solução local para o intervalo $[0, T]$;

(iii) passagem ao limite, que consiste em mostrar que as soluções aproximadas convergem para uma solução do problema original. Nessa etapa, o Teorema de compacidade de Aubin-Lions 1.1.11 e o Lema de Lions 1.1.17 são fundamentais.

(iv) verificação dos dados iniciais, onde comprovamos que a solução obtida na etapa anterior satisfaz os dados iniciais.

Do Teorema 1.1.29 sabemos que as autofunções $\left\{w_{j}\right\}_{j=0}^{\infty}$ do problema

$$
\left\{\begin{array}{l}
\Delta w+\lambda w=0 \quad \text { em } \Omega \\
\left.\frac{\partial w}{\partial \nu}\right|_{\Gamma}=0
\end{array}\right.
$$

constituem um sistema ortonormal completo para $L^{2}(\Omega)$. Além disso, $\left\{w_{j}\right\}_{j \in \mathbb{N}}$ forma uma base para $H_{d}^{1}(\Omega) \cap L^{p}(\Omega)$. Isso decorre da suavidade de $\Omega$.

Para cada $m \in \mathbb{N}, u_{m}=u_{m}(t, x)=\sum_{j=0}^{m} a_{j m}(t) w_{j}(x)$ são as aproximações de Galerkin e representam a projeção da função $u$ sobre o subespaço $H_{m}=\left[w_{0}, \ldots, w_{m}\right]$ gerado pelas $m+1$ primeiras autofunções do problema (3.18). A função $u_{m}$ satisfaz o seguinte sistema de equações diferenciais ordinárias

$$
\left\{\begin{array}{l}
\left\langle\frac{\partial u_{m}}{\partial t}, v\right\rangle-\left\langle d \Delta u_{m}, v\right\rangle+\left\langle f\left(u_{m}\right)-\left|u_{m}\right|^{\alpha-1} u_{m}, v\right\rangle=0, \quad \forall v \in H_{m} \\
u_{m}(0)=u_{0 m} \rightarrow u_{0} \quad \text { em } L^{2}(\Omega), \text { quando } m \rightarrow \infty
\end{array}\right.
$$

para todo $j=0, \ldots, m$.

Pelo Teorema de Caratheodory 1.1.21, o sistema (3.19) possui pelo menos uma solução $u_{m}:\left[0, t_{m}\right) \rightarrow H_{m}=\left[w_{0}, \ldots, w_{m}\right]$ para $T>t_{m}>0$. Como as autofunções do laplaciano são funções suaves com respeito a $x \in \Omega$, podemos assumir que $u_{m} \in C^{1}\left(\left[0, t_{m}\right) ; H_{d}^{1}(\Omega) \cap\right.$ $\left.C^{2}(\bar{\Omega})\right)$.

Na parte $(i i)$, vamos obter estimativas a priori para as aproximações $u_{m}$. Denotemos aqui $u \doteq u_{m}$.

Da equação aproximada (3.19), obtemos

$$
\frac{1}{2} \frac{d}{d t} \int_{\Omega}|u|^{2} d x+d \int_{\Omega}|\nabla u|^{2} d x+\int_{\Omega} f(u) \cdot u d x=\int_{\Omega}|u|^{\alpha+1} d x .
$$


Somando em ambos os lados de (3.20) o termo $\|u\|_{L^{2}(\Omega)}^{2}$ e usando a condição de dissipatividade (3.12) e a Desigualdade de Young (1.3), obtemos

$$
\begin{aligned}
& \frac{1}{2} \frac{d}{d t} \int_{\Omega}|u|^{2} d x+d \int_{\Omega}|\nabla u|^{2} d x+\int_{\Omega}|u|^{2} d x+c_{1} \int_{\Omega}|u|^{p} d x \leqslant \\
& \leqslant c_{3}|\Omega|+\frac{c_{1}}{4} \int_{\Omega}|u|^{p} d x+\left(\frac{4(\alpha+1)}{c_{1} p}\right)^{\frac{\alpha+1}{p-(\alpha+1)}}\left(\frac{p-(\alpha+1)}{p}\right)|\Omega|+\frac{c_{1}}{4} \int_{\Omega}|u|^{p} d x+ \\
& +\left(\frac{8}{c_{1} p}\right)^{\frac{2}{p-2}}\left(\frac{p-2}{p}\right)|\Omega| .
\end{aligned}
$$

Logo,

$$
\frac{d}{d t} \int_{\Omega}|u|^{2} d x+2\left(\int_{\Omega} d|\nabla u|^{2} d x+\int_{\Omega}|u|^{2} d x\right)+c_{1} \int_{\Omega}|u|^{p} d x \leqslant C_{1},
$$

onde

$$
C_{1}=2|\Omega|\left[c_{3}+\left(\frac{4(\alpha+1)}{c_{1} p}\right)^{\frac{\alpha+1}{p-(\alpha+1)}}\left(\frac{p-(\alpha+1)}{p}\right)+\left(\frac{8}{c_{1} p}\right)^{\frac{2}{p-2}}\left(\frac{p-2}{p}\right)\right] .
$$

Integrando a desigualdade (3.21) em relação a $t$, temos

$$
\int_{\Omega}|u(s)|^{2} d x+2 \int_{0}^{t}\|u(s)\|_{H_{d}^{1}(\Omega)} d s+c_{1} \int_{0}^{t} \int_{\Omega}|u(s)|^{p} d x d s \leqslant C_{2},
$$

onde

$$
C_{2}=\int_{\Omega}|u(0)|^{2} d x+C_{1} t
$$

Portanto, as aproximações de Galerkin $u_{m}$ verificam as seguintes estimativas a priori

$$
\sup _{s \in[0, t]} \int_{\Omega}\left|u_{m}(s)\right|^{2} d x \leqslant C_{2}, \quad \int_{0}^{t}\left\|u_{m}(s)\right\|_{H_{d}^{1}(\Omega)}^{2} d s \leqslant \frac{C_{2}}{2}, \quad \int_{0}^{t} \int_{\Omega}\left|u_{m}(s)\right|^{p} d x d s \leqslant \frac{C_{2}}{c_{1}},
$$

onde $C_{2}, \frac{C_{2}}{2}, \frac{C_{2}}{c_{1}}$ são limitados para conjuntos limitados de dados iniciais em $L^{2}(\Omega)$ e para intervalos de tempo limitados. Agora, pelo Teorema de prolongamento de soluções 1.1.23 podemos prolongar a solução $u_{m}(t)$ ao intervalo $[0, T]$.

De (3.23) podemos afirmar

(1) $\left\{u_{m}\right\}$ é uniformemente limitado em $L^{\infty}\left(0, T ; L^{2}(\Omega)\right)$;

(2) $\left\{u_{m}\right\}$ é uniformemente limitado em $L^{2}\left(0, T ; H_{d}^{1}(\Omega)\right)$; 
(3) $\left\{u_{m}\right\}$ é uniformemente limitado em $L^{p}\left(0, T ; L^{p}(\Omega)\right)$.

Além disso,

(4) $\left\{\Delta u_{m}\right\}$ é uniformemente limitado em $L^{2}\left(0, T ; H_{d}^{-1}(\Omega)\right)$;

(5) de (3.16) e (3.17), $\left\{\left(f\left(u_{m}\right)-\left|u_{m}\right|^{\alpha-1} u_{m}\right)\right\}$ é uniformemente limitado em $L^{q}\left(0, T ; L^{q}(\Omega)\right)$;

(6) como $\left\{u_{m}\right\} \subset L^{2}\left(0, T ; \mathcal{H}^{1}(\Omega)\right)$ e da Proposição 1.1.39, a continuidade do operador $\tilde{\Lambda}_{1}$ (definido na Subseção 1.1.1) implica que $\left\{\frac{\partial u_{m}}{\partial \nu}\right\}$ é uniformemente limitado em $L^{2}\left(0, T ; H^{-\frac{1}{2}}(\Gamma)\right.$ ).

Como os espaços $L^{2}\left(0, T ; H_{d}^{-1}(\Omega)\right)$ e $L^{q}\left(0, T ; L^{q}(\Omega)\right)$ estão imersos continuamente em $L^{q}\left(0, T ; H^{-r}(\Omega)\right)$ e

$$
\frac{\partial u_{m}}{\partial t}=d \Delta u_{m}-P_{m}\left(f\left(u_{m}\right)-\left|u_{m}\right|^{\alpha-1} u_{m}\right)
$$

podemos afirmar também que $\left\{\frac{\partial u_{m}}{\partial t}\right\}$ é uniformemente limitado em $L^{q}\left(0, T ; H^{-r}(\Omega)\right)$, onde $P_{m}$ denota o operador projeção.

Agora, por meio do Teorema de Compacidade de Alaoglu 1.1.24 e do Corolário 1.1.25 podemos extrair subsequências convergentes, indicadas por $u_{m}$, tais que

(a) $u_{m} \rightarrow u$ em $L^{2}\left(0, T ; H_{d}^{1}(\Omega)\right)$;

(b) $u_{m} \rightarrow u$ em $L^{p}\left(0, T ; L^{p}(\Omega)\right)$;

(c) $u_{m} \stackrel{*}{\rightarrow} u$ em $L^{\infty}\left(0, T ; L^{2}(\Omega)\right)$;

(d) $\Delta u_{m} \rightarrow \Delta u$ em $L^{2}\left(0, T ; H_{d}^{-1}(\Omega)\right)$;

(e) $\frac{\partial u_{m}}{\partial t} \rightarrow \frac{\partial u}{\partial t}$ em $L^{q}\left(0, T ; H^{-r}(\Omega)\right)$;

(f) $f\left(u_{m}\right)-\left|u_{m}\right|^{\alpha-1} u_{m} \rightarrow w \operatorname{em~} L^{q}\left(0, T ; L^{q}(\Omega)\right)$;

(g) $\frac{\partial u_{m}}{\partial \nu} \rightarrow \frac{\partial u}{\partial \nu}$ em $L^{2}\left(0, T ; H^{-\frac{1}{2}}(\Gamma)\right)$.

Além disso, o Teorema de Compacidade de Aubin-Lions 1.1.11 nos permite extrair uma subsequência, indicada por $\left\{u_{m}\right\}$, tal que $u_{m} \rightarrow u$ em $L^{2}\left(0, T ; L^{2}(\Omega)\right)$. De fato, como $H_{d}^{1}(\Omega) \stackrel{c}{\hookrightarrow} L^{2}(\Omega) \hookrightarrow H^{-r}(\Omega),\left\{u_{m}\right\}$ é uniformemente limitada em $L^{2}\left(0, T ; H_{d}^{1}(\Omega)\right)$ e $\left\{\frac{\partial u_{m}}{\partial t}\right\}$ é uniformemente limitada em $L^{q}\left(0, T ; H^{-r}(\Omega)\right)$ segue do Teorema de Lions a convergência desejada.

Fazendo $m \rightarrow \infty$ em (3.19) obtemos a seguinte igualdade

$$
\frac{\partial u}{\partial t}=d \Delta u-w
$$

no sentido de distribuição do espaço $\mathcal{D}^{\prime}\left(0, T ; H^{-r}(\Omega)\right)$.

Precisamos agora verificar que $w=f(u)-|u|^{\alpha-1} u$.

Como $u_{m} \rightarrow u$ em $L^{2}\left(0, T ; L^{2}(\Omega)\right)$, pelo Lema 1.1.28 existe uma subsequência $\left\{u_{m_{j}}\right\}$ que converge pontualmente para $u$ quase sempre em $[0, T] \times \Omega$, isto é, $u_{m_{j}}(t, x) \rightarrow u(t, x)$ quase sempre para $(t, x) \in[0, T] \times \Omega$. Da continuidade de $f$ resulta que $f\left(u_{m_{j}}(t, x)\right) \rightarrow f(u(t, x))$ quase sempre para $(t, x) \in[0, T] \times \Omega$. 
Agora, da limitação de $f\left(u_{m}\right)$ em $L^{q}\left(0, T ; L^{q}(\Omega)\right)$ podemos aplicar o Lema de Lions 1.1.17, donde $f\left(u_{m}\right) \rightarrow f(u)$ em $L^{q}\left(0, T ; L^{q}(\Omega)\right)$. O mesmo vale para o termo $|u|^{\alpha-1} u$.

Portanto $w=f(u)-|u|^{\alpha-1} u$.

Vejamos agora que $u(0)=u_{0}$. De fato, tomemos $\phi \in C^{1}\left([0, T] ; H_{d}^{1}(\Omega) \cap L^{p}(\Omega)\right)$ com $\phi(T)=0$ e observemos que $\phi \in L^{2}\left(0, T ; H_{d}^{1}(\Omega)\right) \cap L^{p}\left(0, T ; L^{p}(\Omega)\right)$. Fazendo o produto por $\phi(t)$ na equação aproximada (3.24) e na equação limite, obtemos

$$
\left\langle\frac{\partial u}{\partial t}, \phi\right\rangle+B(u, \phi)+\left\langle f(u)-|u|^{\alpha-1} u, \phi\right\rangle=0,
$$

onde $B(u, \phi)=\int_{\Omega} u(s) \phi(s) d x$, e integrando por partes na variável $t$ ficamos com

$$
\int_{0}^{T}\left[-\left\langle u, \phi^{\prime}\right\rangle+B(u, \phi)\right] d s+\int_{0}^{T}\left\langle f(u)-|u|^{\alpha-1} u, \phi\right\rangle d s=\langle u(0), \phi(0)\rangle .
$$

Analogamente na equação aproximada

$$
\int_{0}^{T}\left[-\left\langle u_{m}, \phi^{\prime}\right\rangle+B\left(u_{m}, \phi\right)\right] d s+\int_{0}^{T}\left\langle f\left(u_{m}\right)-\left|u_{m}\right|^{\alpha-1} u_{m}, \phi\right\rangle d s=\left\langle u_{m}(0), \phi(0)\right\rangle .
$$

Fazendo $m \rightarrow \infty$ em (3.25) concluímos que

$$
\int_{0}^{T}\left[-\left\langle u, \phi^{\prime}\right\rangle+B(u, \phi)\right] d s+\int_{0}^{T}\left\langle f(u)-|u|^{\alpha-1} u, \phi\right\rangle d s=\left\langle u_{0}, \phi(0)\right\rangle,
$$

pois pelo Lema 1.1.30 $u_{m}(0)=P_{m} u_{0} \rightarrow u_{0}$. Portanto, $u(0)=u_{0}$.

O próximo resultado é consequência do Lema 1.1.19

Proposição 3.1.3. Se $u \in L^{p}\left(0, T ; L^{p}(\Omega)\right) \cap L^{2}\left(0, T ; H_{d}^{1}(\Omega)\right)$ é uma solução fraca de (3.10), então

(i) $u \in C\left([0, T] ; L^{2}(\Omega)\right)$;

(ii) $\|u(\cdot)\|_{L^{2}(\Omega)}^{2}$ é absolutamente contínua em $[0, T]$ e

$$
\frac{1}{2} \frac{d}{d t}\|u(t)\|_{L^{2}(\Omega)}^{2}+d\langle\nabla u(t), \nabla u(t)\rangle+\left\langle f(u(t))-|u(t)|^{\alpha-1} u(t), u(t)\right\rangle=0,
$$

para quase todo $t \in[0, T]$.

Corolário 3.1.4. Seja $C_{1}$ o número positivo dado em (3.22). Se $u \in L^{p}\left(0, T ; L^{p}(\Omega)\right) \cap$ $L^{2}\left(0, T ; H_{d}^{1}(\Omega)\right)$ é uma solução fraca de (3.10), então para $t \geqslant 0$ 
(i)

$$
\|u(t)\|_{L^{2}(\Omega)}^{2} \leqslant\|u(0)\|_{L^{2}(\Omega)}^{2} e^{-2 t}+\frac{C_{1}}{2}
$$

(ii)

$$
2 \int_{t}^{t+1}\|u(s)\|_{H_{d}^{1}(\Omega)}^{2} d s+c_{1} \int_{t}^{t+1}\|u(s)\|_{L^{p}(\Omega)}^{p} d s \leqslant\|u(0)\|_{L^{2}(\Omega)}^{2} e^{-2 t}+\frac{3 C_{1}}{2} .
$$

Demonstração: Se $u \in L^{p}\left(0, T ; L^{p}(\Omega)\right) \cap L^{2}\left(0, T ; H_{d}^{1}(\Omega)\right)$ é uma solução fraca de $(3.10)$, então de (3.26), obtemos

$$
\frac{d}{d t} \int_{\Omega}|u(t)|^{2} d x+2\|u(t)\|_{H_{d}^{1}(\Omega)}^{2}+c_{1}\|u(t)\|_{L^{p}(\Omega)}^{p} \leqslant C_{1}
$$

Em particular,

$$
\frac{d}{d t} \int_{\Omega}|u|^{2}+2 \int_{\Omega}|u|^{2} d x \leqslant C_{1}
$$

e portanto,

$$
\|u(t)\|_{L^{2}(\Omega)}^{2} \leqslant\|u(0)\|_{L^{2}(\Omega)}^{2} e^{-2 t}+\frac{C_{1}}{2}
$$

Quando integramos (3.27) em $[t, t+1]$, obtemos

$$
\begin{aligned}
& \int_{\Omega}|u(t+1)|^{2} d x-\int_{\Omega}|u(t)|^{2} d x+2 \int_{t}^{t+1}\|u(s)\|_{H_{d}^{1}(\Omega)}^{2} d s+c_{1} \int_{t}^{t+1} \int_{\Omega}|u(s)|^{p} d x d s \leqslant \\
& \leqslant C_{1} .
\end{aligned}
$$

Logo,

$$
2 \int_{t}^{t+1}\|u(s)\|_{H_{d}^{1}(\Omega)}^{2} d s+c_{1} \int_{t}^{t+1} \int_{\Omega}|u(s)|^{p} d x d s \leqslant\|u(0)\|_{L^{2}(\Omega)}^{2} e^{-2 t}+\frac{3 C_{1}}{2} .
$$

Observemos que se consideramos o dado inicial $u_{0} \in H_{d}^{1}(\Omega)$, então é possível obter mais uma estimativa a priori. De fato, fazendo o produto da equação (3.10) por $-\Delta u$ e 
integrando em $\Omega$, obtemos

$$
-\int_{\Omega} u_{t} \Delta u d x+d\|\Delta u\|_{L^{2}(\Omega)}^{2}-\int_{\Omega} f(u) \Delta u d x+\int_{\Omega}|u|^{\alpha-1} u \Delta u d x=0 .
$$

Observemos que

$$
-\int_{\Omega} u_{t} \Delta u d x=\frac{1}{2} \frac{d}{d t}\|\nabla u\|_{L^{2}(\Omega)}^{2}
$$

Isso é verdade se $u$ é suficientemente regular, digamos, se $u \in C^{1}\left([0, T] ; C^{2}(\bar{\Omega})\right)$. Essa propriedade é válida se $u=u_{m}$ é uma aproximação de Galerkin arbitrária. Logo, toda derivada de $u_{m}$ satisfaz (3.29) e podemos prosseguir com os nossos cálculos sob a condição que $u$ é suficientemente regular ou $u=u_{m}$ é uma aproximação de Galerkin.

Usando integração de partes e da hipótese (3.11) segue que

$$
-\int_{\Omega} f(u) \Delta u d x=\int_{\Omega} \sum_{j=1}^{n} \frac{\partial f(u)}{\partial u}\left(\frac{\partial u}{\partial x_{j}}\right)^{2} d x \geqslant c_{f} \int_{\Omega}|\nabla u|^{2} d x
$$

e usando a Desigualdade de Young (1.3), temos

$$
\begin{aligned}
& \int_{\Omega}|u|^{\alpha-1} u \Delta u d x \leqslant \frac{1}{2 d} \int_{\Omega}|u|^{2 \alpha} d x+\frac{d}{2} \int_{\Omega}|\Delta u|^{2} d x \leqslant \frac{\alpha}{2 d}\|u\|_{L^{2}(\Omega)}^{2}+ \\
& +\frac{1}{2 d}(1-\alpha)|\Omega|+\frac{d}{2} \int_{\Omega}|\Delta u|^{2} d x .
\end{aligned}
$$

Aplicando (3.29), (3.30) e (3.31) em (3.28), obtemos

$$
\frac{d}{d t}\|\nabla u\|_{L^{2}(\Omega)}^{2}+d\|\Delta u\|_{L^{2}(\Omega)}^{2}+2 c_{f}\|\nabla u\|_{L^{2}(\Omega)}^{2} \leqslant \frac{\alpha}{d}\|u\|_{L^{2}(\Omega)}^{2}+\frac{1}{d}(1-\alpha)|\Omega| .
$$

Usando a desigualdade $\|\Delta u\|_{L^{2}(\Omega)}^{2} \geqslant \lambda_{1}\|\nabla u\|_{L^{2}(\Omega)}^{2}\left(\lambda_{1}\right.$ é o primeiro autovalor positivo de $-\Delta$ com condição de Neumann na fronteira) em (3.32) e fazendo alguns cálculos simples ficamos com

$$
\frac{d}{d t}\left(\|\nabla u\|_{L^{2}(\Omega)}^{2} e^{d \lambda_{1} t}\right) \leqslant \frac{\alpha}{d}\|u(0)\|_{L^{2}(\Omega)}^{2} e^{-t} e^{\lambda_{1} d t}+\frac{C_{1} \alpha}{2 d} e^{d \lambda_{1} t}+\frac{(1-\alpha)}{d}|\Omega| e^{d \lambda_{1} t}
$$


Logo, integrando (3.33) em $t$ resulta que

$$
\begin{aligned}
\|\nabla u(t)\|_{L^{2}(\Omega)}^{2} & \leqslant\|\nabla u(0)\|_{L^{2}(\Omega)}^{2} e^{-d \lambda_{1} t}+\frac{C_{1} \alpha}{2 d^{2} \lambda_{1}}+\frac{(1-\alpha)|\Omega|}{d^{2} \lambda_{1}}+ \\
& +\frac{\alpha}{d\left(d \lambda_{1}-1\right)}\|u(0)\|_{L^{2}(\Omega)}^{2} e^{-t} .
\end{aligned}
$$

Como nosso interesse será em valores grandes de $d$ podemos assumir que $d>1$ e $d \lambda_{1}>2$. Assim,

$$
\|\nabla u(t)\|_{L^{2}(\Omega)}^{2} \leqslant\|\nabla u(0)\|_{L^{2}(\Omega)}^{2} e^{-d \lambda_{1} t}+C_{1} \alpha+(1-\alpha)|\Omega|+\alpha\|u(0)\|_{L^{2}(\Omega)}^{2} e^{-t}
$$

Portanto, de (3.35) segue que $\|\nabla u\|_{L^{\infty}\left(0, T ; L^{2}(\Omega)\right)}<+\infty$, para $T>0$. Além disso, como $\|u\|_{L^{\infty}\left(0, T ; L^{2}(\Omega)\right)}<+\infty$ (Corolário 3.1.4), resulta que $\|u\|_{L^{\infty}\left(0, T ; H_{d}^{1}(\Omega)\right)}<+\infty$ para todo $T>0$.

Para cada $d>1$ tal que $d \lambda_{1}>2$, introduzimos os espaços $\mathcal{F}_{l o c, d}^{+}, \mathcal{F}_{b, d}^{+}$e a topologia $\Theta_{l o c, d}^{+}$de acordo com o roteiro da Seção 2.2.

$$
\mathcal{F}_{l o c, d}^{+}=\left\{u: u \in L_{l o c}^{\infty}\left(\mathbb{R}_{+} ; H_{d}^{1}(\Omega)\right) \cap L_{l o c}^{p}\left(\mathbb{R}_{+} ; L^{p}(\Omega)\right) \text { e } \partial_{t} v \in L_{l o c}^{q}\left(\mathbb{R}_{+} ; H^{-r}(\Omega)\right)\right\},
$$

onde $r=\max \left\{1, n\left(\frac{1}{2}-\frac{1}{p}\right)\right\}$.

Sejam $p>1$ e $E$ um espaço de Banach. Consideremos o espaço de Banach $L_{b}^{p}\left(\mathbb{R}_{+} ; E\right)$ munido da norma

$$
\|v\|_{L_{b}^{p}\left(\mathbb{R}_{+} ; E\right)}^{p}=\sup _{t \in \mathbb{R}_{+}} \int_{t}^{t+1}\|v(s)\|_{E}^{p} d s .
$$

O espaço $\mathcal{F}_{b, d}^{+}$é definido da seguinte maneira

$$
\begin{aligned}
\mathcal{F}_{b, d}^{+}= & \left\{u \in \mathcal{F}_{l o c, d}^{+}: u \in L^{\infty}\left(\mathbb{R}_{+} ; H_{d}^{1}(\Omega)\right) \cap L_{b}^{p}\left(\mathbb{R}_{+} ; L^{p}(\Omega)\right) \text { e } \partial_{t} u \in L_{b}^{q}\left(\mathbb{R}^{+} ; H^{-r}(\Omega)\right)\right. \\
& \left.\operatorname{com}\|u\|_{\mathcal{F}_{b, d}^{+}}<+\infty\right\}
\end{aligned}
$$

onde $\|u\|_{\mathcal{F}_{b, d}^{+}} \doteq\|u\|_{L^{\infty}\left(\mathbb{R}_{+} ; H_{d}^{1}(\Omega)\right)}+\|u\|_{L_{b}^{p}\left(\mathbb{R}_{+} ; L^{p}(\Omega)\right)}+\left\|\partial_{t} u\right\|_{L_{b}^{q}\left(\mathbb{R}^{+} ; H^{-r}(\Omega)\right)}$.

A topologia $\Theta_{l o c, d}^{+}$atuando no espaço $\mathcal{F}_{l o c, d}^{+}$é definida em termos de sequências da seguinte forma: $\left\{u_{m}\right\} \subset \mathcal{F}_{\text {loc,d }}^{+}$converge para uma função $u \in \mathcal{F}_{l o c, d}^{+}$quando para todo intervalo $[0, T] \subset \mathbb{R}_{+}$temos

(i) $u_{m} \stackrel{*}{\rightarrow} u$ em $L^{\infty}\left(0, T ; H_{d}^{1}(\Omega)\right)$,

(ii) $u_{m} \rightarrow u$ em $L^{p}\left(0, T ; L^{p}(\Omega)\right)$ e

(iii) $\partial_{t} u_{m} \rightarrow \partial_{t} u$ em $L^{q}\left(0, T ; H^{-r}(\Omega)\right)$. 
O espaço $\mathcal{F}_{l o c, d}^{+}$munido da topologia $\Theta_{l o c, d}^{+}$é um espaço topológico Hausdorff, FrechetUryshon e possui uma base enumerável. De fato, tomemos $z \in \mathcal{F}_{l o c, d}^{+}$e indiquemos por $\tau_{1}$ : a topologia gerada pela norma do espaço $\left(L^{\infty}\left(0, T ; H_{d}^{1}(\Omega)\right),\|\cdot\|_{1}\right)$; $\tau_{2}$ : a topologia em $\left(L^{p}\left(0, T ; L^{p}(\Omega)\right),\|\cdot\|_{2}\right)$ e $\tau_{3}$ : a topologia em $\left(L^{q}\left(0, T ; H^{-r}(\Omega)\right),\|\cdot\|_{3}\right)$.

Consideremos $\left\{B_{1}\left(z, \frac{1}{n}\right): n \in \mathbb{N}\right\},\left\{B_{2}\left(z, \frac{1}{n}\right): n \in \mathbb{N}\right\}$ e $\left\{B_{3}\left(\partial_{t} z, \frac{1}{n}\right): n \in \mathbb{N}\right\}$, onde $B_{i}\left(y, \frac{1}{n}\right)=\left\{\tilde{y}:\|y-\tilde{y}\|_{i}<\frac{1}{n}\right\}$ para $i=1,2,3$. Cada um desses conjuntos constitui uma coleção enumerável de vizinhanças abertas de $z$ nas topologias $\tau_{1}, \tau_{2}$, respectivamente, e de $\partial_{t} z$ na topologia $\tau_{3}$. Façamos a reunião desses conjuntos abertos $\cup_{j=1}^{2} B_{j}\left(z, \frac{1}{n}\right) \cup B_{3}\left(\partial_{t} z, \frac{1}{n}\right)=\mathfrak{F}(z)$. Observemos que essa reunião é enumerável, ou seja, podemos considerar $\mathfrak{F}(z)=\left\{F_{n}: n \in \mathbb{N}\right\}$.

Seja $I \subset \mathbb{N}$ um subconjunto finito tal que $\left\{z, \partial_{t} z\right\} \subset V_{I}=\left(\cap_{n \in I} F_{n}^{z}\right) \cup\left(\cap_{n \in I} F_{n}^{\partial_{t} z}\right)$, onde $F_{n}^{z}$ denota os elementos de $\mathfrak{F}(z)$ contendo $z$ e $F_{n}^{\partial_{t} z}$ são os elementos de $\mathfrak{F}(z)$ contendo $\partial_{t} z$. Definamos $\tilde{B}(z)=\left\{V_{I}: I \subset \mathbb{N}, I\right.$ é finito $\}$. Este conjunto é um elemento básico da topologia $\Theta_{l o c, d}^{+}$contendo z. Logo, a topologia $\Theta_{l o c, d}^{+}$é gerada pela reunião dos conjuntos da forma $\cup_{z \in \mathcal{F}_{l o c, d}^{+}} \tilde{B}(z)$.

Se $z_{n} \rightarrow z$ em $\Theta_{l o c, d}^{+}$, segue da Proposição 1.2 .9 que o espaço topológico $\mathcal{F}_{\text {loc,d }}^{+}$munido da topologia $\Theta_{l o c, d}^{+}$é Hausdorff. Além disso, como existe uma base enumerável local para todo elemento de $\mathcal{F}_{l o c, d}^{+}$este espaço é Frechet-Uryshon.

O espaço de trajetórias $\mathcal{K}_{d}^{+}$da equação (3.10) é descrito por

$$
\begin{aligned}
\mathcal{K}_{d}^{+}= & \left\{u: u \in L^{\infty}\left(0, T ; H_{d}^{1}(\Omega)\right) \cap L^{p}\left(0, T ; L^{p}(\Omega)\right) \text { é solução fraca de }(3.10)\right. \\
& \text { para todo } T>0\} .
\end{aligned}
$$

Pelo Teorema 3.1.2, temos que $\mathcal{K}_{d}^{+} \neq \emptyset$. Além disso, se $u \in \mathcal{K}_{d}^{+}$, então $\partial_{t} u \in L_{\text {loc }}^{q}\left(\mathbb{R}_{+} ; H^{-r}(\Omega)\right)$.

Proposição 3.1.5. Se $\mathcal{K}_{d}^{+}$é o espaço de trajetórias da equação (3.10), então $\mathcal{K}_{d}^{+}$é fechado na topologia $\Theta_{l o c, d}^{+}$.

Demonstração: Seja $\left\{u_{m}\right\}_{m=1}^{\infty} \subset \mathcal{K}_{d}^{+}$uma sequência tal que $u_{m} \rightarrow u$ em $\Theta_{\text {loc,d }}^{+} \operatorname{com} u \in$ $\mathcal{F}_{l o c, d}^{+}$. Vamos verificar que $u \in \mathcal{K}_{d}^{+}$.

Da definição da topologia $\Theta_{l o c, d}^{+}$segue que

(i) $u_{m} \stackrel{*}{\rightarrow} u$ em $L^{\infty}\left(0, T ; H_{d}^{1}(\Omega)\right)$;

(ii) $u_{m} \rightarrow u$ em $L^{2}\left(0, T ; H_{d}^{1}(\Omega)\right)$;

(iii) $u_{m} \rightarrow u$ em $L^{p}\left(0, T ; L^{p}(\Omega)\right) \mathrm{e}$

(iv) $\partial_{t} u_{m} \rightarrow \partial_{t} u$ em $L^{q}\left(0, T ; H^{-r}(\Omega)\right)$.

Como toda sequência fracamente convergente é limitada (Proposição 1.1.16) temos que a sequência $\left\{u_{m}\right\}_{m=1}^{\infty}$ é limitada em cada um dos espaços $L^{\infty}\left(0, T ; H_{d}^{1}(\Omega)\right), L^{2}\left(0, T ; H_{d}^{1}(\Omega)\right)$, $L^{p}\left(0, T ; L^{p}(\Omega)\right)$ e $\left\{\partial_{t} u_{m}\right\}_{m=1}^{\infty}$ é limitada em $L^{q}\left(0, T ; H^{-r}(\Omega)\right)$. Em virtude do Teorema 1.1.24 
e do Corolário 1.1.25 podemos extrair subsequências convergentes. Além disso, a desigualdade (3.12) implica que a sequência $f\left(u_{m}\right)$ é limitada em $L^{q}\left(0, T ; L^{q}(\Omega)\right)$. Logo existe uma subsequência fracamente convergente, indicada por $f\left(u_{m}\right)$, tal que $f\left(u_{m}\right) \rightarrow \zeta$ para algum $\zeta \in L^{q}\left(0, T ; L^{q}(\Omega)\right)$. O mesmo vale para o termo $\left|u_{m}\right|^{\alpha-1} u_{m}$. E $f\left(u_{m}\right)-\left|u_{m}\right|^{\alpha-1} u_{m} \rightarrow \omega$.

É claro que $u_{m}$ é solução fraca de (3.10), isto é,

$$
\frac{\partial u_{m}}{\partial t}=d \Delta u_{m}-f\left(u_{m}\right)+\left|u_{m}\right|^{\alpha-1} u_{m}
$$

De (3.36) e do que observamos acima, obtemos que a função $u$ satisfaz a equação diferencial

$$
\partial_{t} u=d \Delta u-\omega
$$

no sentido de distribuição.

Do Teorema de Compacidade de Aubin-Lions temos também $u_{m} \rightarrow u$ em $L^{2}\left(0, T ; L^{2}(\Omega)\right)$ e pelo Lema 1.1 .28 existe uma subsequência $\left\{u_{m_{j}}\right\} \subset\left\{u_{m}\right\}$ tal que $u_{m_{j}}(t, x) \rightarrow u(t, x)$ quase sempre para $(t, x) \in[0, T] \times \Omega$. Da continuidade da função $f$ podemos afirmar que $f\left(u_{m_{j}}(t, x)\right) \rightarrow f(u(t, x))$ quase sempre para $(t, x) \in[0, T] \times \Omega$. Agora, da limitação de $f\left(u_{m}\right)$ em $L^{q}\left(0, T ; L^{q}(\Omega)\right)$ e do Lema de Lions 1.1 .17 concluímos que $f\left(u_{m}\right) \rightarrow f(u)$ em $L^{q}\left(0, T ; L^{q}(\Omega)\right)$. O mesmo vale para o termo $|u|^{\alpha-1} u$.

$$
\log 0 \omega=f(u)-|u|^{\alpha-1} u \text {. }
$$

Portanto $u \in \mathcal{K}_{d}^{+}$.

Definamos a aplicação $T(t): \mathcal{F}_{l o c, d}^{+} \rightarrow \mathcal{F}_{l o c, d}^{+}$por $T(t) u(s)=u(t+s)$ e consideremos o semigrupo translação $\{T(t): t \geqslant 0\}$. Como a equação (3.10) é autônoma decorre imediatamente que o espaço de trajetórias $\mathcal{K}_{d}^{+}$é invariante pelo semigrupo $\{T(t)\}$, logo podemos considerar $T(t): \mathcal{K}_{d}^{+} \rightarrow \mathcal{K}_{d}^{+}$.

O semigrupo $\{T(t): t \geqslant 0\}$ é contínuo com respeito a topologia $\Theta_{l o c, d}^{+}$. De fato, se $u_{n} \rightarrow u$ em $\Theta_{l o c, d}^{+}$, então $\Pi_{[0, T]} u_{n} \rightarrow \Pi_{[0, T]} u$ em $\Theta_{[0, T], d}$ para todo $T>0$. Em particular, $\Pi_{[0, T+t]} u_{n} \rightarrow \Pi_{[0, T+t]} u$ em $\Theta_{[0, T+t], d}$ para todo $t \geqslant 0$. Como $T(t): \mathcal{F}_{d}(0, T+t) \rightarrow \mathcal{F}_{d}(0, T)$ é uma aplicação contínua temos $\Pi_{[0, T]} T(t) u_{n} \rightarrow \Pi_{[0, T]} T(t) u$ em $\Theta_{[0, T], d}$ para todo $T>0$, isto é, $T(t) u_{n} \rightarrow T(t) u$ em $\Theta_{l o c, d}^{+}$.

Proposição 3.1.6. Se $\mathcal{K}_{d}^{+}$é o espaço de trajetórias de (3.10), então para toda função $u \in \mathcal{K}_{d}^{+}$ existem constantes positivas $K_{1}$ e $K_{2}$ tais que

$$
\|T(t) u\|_{\mathcal{F}_{b, d}^{+}} \leqslant K_{1}\|u(0)\|_{H_{d}^{1}(\Omega)}^{2} e^{-t}+K_{2}
$$


para todo $t \geqslant 0$.

Demonstração: Do Corolário 3.1.4, item $(i)$, para $s \geqslant 0$ temos

$$
\|T(t) u(s)\|_{L^{2}(\Omega)}^{2} \leqslant\|u(0)\|_{L^{2}(\Omega)}^{2} e^{-2(t+s)}+\frac{C_{1}}{2} \leqslant\|u(0)\|_{L^{2}(\Omega)}^{2} e^{-2 t}+\frac{C_{1}}{2} \leqslant\|u(0)\|_{L^{2}(\Omega)}^{2} e^{-2 t}+\frac{C_{1}}{2} .
$$

Portanto,

$$
\|T(t) u\|_{L^{\infty}\left(\mathbb{R}_{+}, L^{2}(\Omega)\right)}^{2} \leqslant\|u(0)\|_{L^{2}(\Omega)}^{2} e^{-t}+\frac{C_{1}}{2}
$$

para $t \geqslant 0$

E o Corolário 3.1.4, item (ii), implica que

$$
\begin{aligned}
& 2\|T(t) u\|_{L^{2}\left(0,1 ; H_{d}^{1}(\Omega)\right)}^{2}+c_{1}\|T(t) u\|_{L^{p}\left(0,1 ; L^{p}(\Omega)\right)}^{p}=2 \int_{0}^{1}\|u(t+s)\|_{H_{d}^{1}(\Omega)}^{2} d s+ \\
& \quad+c_{1} \int_{0}^{1}\|u(t+s)\|_{L^{p}(\Omega)}^{p} d s=2 \int_{t}^{t+1}\|u(s)\|_{H_{d}^{1}(\Omega)}^{2} d s+c_{1} \int_{t}^{t+1}\|u(s)\|_{L^{p}(\Omega)}^{p} d s \leqslant \\
& \quad \leqslant\|u(0)\|_{L^{2}(\Omega)}^{2} e^{-2 t}+\frac{3 C_{1}}{2}
\end{aligned}
$$

para $t \geqslant 0$.

De (3.35) segue que

$$
\begin{aligned}
\|T(t) \nabla u\|_{L^{\infty}\left(\mathbb{R}_{+}, L^{2}(\Omega)\right)}^{2} & \leqslant\|\nabla u(0)\|_{L^{2}(\Omega)}^{2} e^{-d \lambda_{1} t}+C_{1} \alpha+(1-\alpha)|\Omega|+\alpha\|u(0)\|_{L^{2}(\Omega)}^{2} e^{-t} \\
& \leqslant\|\nabla u(0)\|_{L^{2}(\Omega)}^{2} e^{-t}+C_{1} \alpha+(1-\alpha)|\Omega|+\|u(0)\|_{L^{2}(\Omega)}^{2} e^{-t} \\
& \leqslant\|u(0)\|_{H_{d}^{1}(\Omega)}^{2} e^{-t}+C_{1} \alpha+(1-\alpha)|\Omega|
\end{aligned}
$$

para $t \geqslant 0$.

De (3.38) e (3.40), obtemos

$$
\|T(t) u\|_{L^{\infty}\left(\mathbb{R}_{+}, H_{d}^{1}(\Omega)\right)}^{2} \leqslant\|u(0)\|_{H_{d}^{1}(\Omega)}^{2} e^{-t}+\max \left\{\frac{C_{1}}{2}, C_{1} \alpha+(1-\alpha)|\Omega|\right\},
$$

para $t \geqslant 0$.

Agora vamos estimar $\left\|\partial_{t} T(t) u\right\|_{L^{q}\left(0,1 ; H^{-r}(\Omega)\right)}$.

$$
\begin{aligned}
& \left\|\partial_{t} T(t) u\right\|_{L^{q}\left(0,1 ; H^{-r}(\Omega)\right)}=\left(\int_{0}^{1}\left\|\partial_{t} u(t+s)\right\|_{H^{-r}(\Omega)}^{q} d s\right)^{\frac{1}{q}}=\left(\int_{t}^{t+1}\left\|\partial_{t} u(s)\right\|_{H^{-r}(\Omega)}^{q} d s\right)^{\frac{1}{q}} \leqslant \\
& \leqslant\left(\int_{t}^{t+1}\|d \Delta u(s)\|_{H^{-r}(\Omega)}^{q} d s\right)^{\frac{1}{q}}+\left(\int_{t}^{t+1}\|f(u(s))\|_{H^{-r}(\Omega)}^{q} d s\right)^{\frac{1}{q}}+\left(\int_{t}^{t+1}\left\|\left.u(s)\right|^{\alpha}\right\|_{H^{-r}(\Omega)}^{q} d s\right)^{\frac{1}{q}}
\end{aligned}
$$




$$
\leqslant k_{1} d\left(\int_{t}^{t+1}\|\Delta u(s)\|_{H_{d}^{-1}(\Omega)}^{q} d s\right)^{\frac{1}{q}}+k_{2}\left(\int_{t}^{t+1}\|f(u(s))\|_{L^{q}(\Omega)}^{q} d s\right)^{\frac{1}{q}}+k_{3}\left(\int_{t}^{t+1}\left\||u(s)|^{\alpha}\right\|_{L^{q}(\Omega)}^{q} d s\right)^{\frac{1}{q}}
$$

onde $k_{1}, k_{2}$ e $k_{3}$ são constantes de imersão.

Vamos analisar separadamente a primeira parcela do lado direito de (3.42).

Usando (3.15) podemos escrever

$$
\begin{aligned}
& \left(\int_{t}^{t+1}\|\Delta u(s)\|_{H_{d}^{-1}(\Omega)}^{q} d s\right)^{\frac{1}{q}} \leqslant\left(\int_{t}^{t+1} \frac{1}{d^{\frac{q}{2}}}\|\nabla u(s)\|_{L^{2}(\Omega)}^{q} d s\right)^{\frac{1}{q}} \\
& \leqslant \frac{1}{\sqrt{d}}\left(\int_{t}^{t+1}\|\nabla u(s)\|_{L^{2}(\Omega)}^{q} d s\right)^{\frac{1}{q}} \leqslant \frac{1}{\sqrt{d}}\left(\int_{t}^{t+1}\|\nabla u(s)\|_{L^{2}(\Omega)}^{2} d s\right)^{\frac{1}{2}} .
\end{aligned}
$$

De (3.43) e do Corolário 3.1.4, item (ii), obtemos que

$$
d^{2}\left(\int_{t}^{t+1}\|\Delta u(s)\|_{H_{d}^{-1}(\Omega)}^{q} d s\right)^{\frac{2}{q}} \leqslant d \int_{t}^{t+1}\|\nabla u(s)\|_{L^{2}(\Omega)}^{2} d s \leqslant \frac{3 C_{1}}{4}+\frac{1}{2}\|u(0)\|_{L^{2}(\Omega)}^{2} e^{-2 t} .
$$

Aplicando (3.44), a hipótese (3.13) e as estimativas obtidas no Corolário 3.1.4 em (3.42) ficamos com

$$
\begin{aligned}
& \left\|\partial_{t} T(t) u\right\|_{L^{q}\left(0,1 ; H^{-r}(\Omega)\right)} \leqslant k_{1}\left(\sqrt{\frac{3 C_{1}}{4}}+\sqrt{\frac{1}{2}}\|u(0)\|_{L^{2}(\Omega)} e^{-t}\right)+ \\
& +k_{2} c_{2}^{\frac{1}{q}}\left(\frac{1}{c_{1}}\left[\|u(0)\|_{L^{2}(\Omega)}^{2} e^{-2 t}+\frac{3 C_{1}}{2}\right]+|\Omega|\right)^{\frac{1}{q}}+k_{3}\left(\frac{\alpha q}{2}\|u(0)\|_{L^{2}(\Omega)}^{2} e^{-2 t}+\frac{\alpha q C_{1}}{4}+\frac{2-\alpha q}{2}|\Omega|\right)^{\frac{1}{q}} \\
& \leqslant k_{1}\left(\sqrt{\frac{3 C_{1}}{4}}+\sqrt{\frac{1}{2}}\|u(0)\|_{L^{2}(\Omega)} e^{-t}\right)+k_{2} c_{2}^{\frac{1}{q}}\left(\frac{1}{c_{1}}\left[\|u(0)\|_{L^{2}(\Omega)}^{2} e^{-2 t}+\frac{3 C_{1}}{2}\right]+|\Omega|+1\right)+ \\
& +k_{3}\left(\frac{\alpha q}{4}\|u(0)\|_{L^{2}(\Omega)}^{2} e^{-2 t}+\frac{\alpha q C_{1}}{4}+\frac{2-\alpha q}{2}|\Omega|+1\right)
\end{aligned}
$$

De (3.45), obtemos constantes positivas $\tilde{K}_{1}$ e $\tilde{K}_{2}$ tais que

$$
\left\|\partial_{t} T(t) u\right\|_{L^{q}\left(0,1 ; H^{-r}(\Omega)\right)} \leqslant \tilde{K}_{1}\|u(0)\|_{L^{2}(\Omega)}^{2} e^{-t}+\tilde{K}_{2}
$$

para $t \geqslant 0$, onde

$$
\tilde{K}_{1}=k_{1} \sqrt{\frac{1}{2}}+\frac{k_{2} c_{2}^{\frac{1}{q}}}{c_{1}}+\frac{k_{3} \alpha q}{4}
$$


$\mathrm{e}$

$$
\tilde{K}_{2}=k_{1} \sqrt{\frac{3 C_{1}}{4}}+\frac{k_{2} c_{2}^{\frac{1}{q}}}{c_{1}} \frac{3 C_{1}}{2}+k_{2} c_{2}^{\frac{1}{q}}(|\Omega|+1)+k_{3}\left(\frac{\alpha q C_{1}}{4}+\frac{2-\alpha q}{2}|\Omega|+1\right)+k_{1} \sqrt{\frac{1}{2}} .
$$

Portanto, de (3.39), (3.41) e (3.46) existem constantes positivas $K_{1}$ e $K_{2}$ tais que

$$
\|T(t) u\|_{\mathcal{F}_{b, d}^{+}} \leqslant K_{1}\|u(0)\|_{H_{d}^{1}(\Omega)}^{2} e^{-t}+K_{2},
$$

para $t \geqslant 0$, onde

$$
K_{1}=\max \left\{1, \frac{1}{c_{1}}, \tilde{K}_{1}\right\}
$$

e

$$
K_{2}=\max \left\{\frac{3 C_{1}}{2 c_{1}}+1, \tilde{K}_{2}, \max \left\{\frac{C_{1}}{2}, C_{1} \alpha+(1-\alpha)|\Omega|\right\}+1\right\} .
$$

Observação 3.1.2. Da Proposição 3.1.6 decorre que $\mathcal{K}_{d}^{+} \subset \mathcal{F}_{b, d}^{+}$.

Proposição 3.1.7. Se $f$ satisfaz (3.11)-(3.13) então para cada $d>1$ tal que $d \lambda_{1}>2$ o problema (3.10) possui um atrator de trajetórias $\mathfrak{A}_{d}$ e existe uma constante $K>0$, independente de d, tal que $\mathfrak{A}_{d} \subset\left\{\tilde{u}:\|\tilde{u}\|_{\mathcal{F}_{b, d}^{+}} \leqslant K\right\}$.

Demonstração: Da desigualdade (3.37) segue que o conjunto

$$
P=\left\{u \in \mathcal{K}_{d}^{+}:\|u\|_{\mathcal{F}_{b, d}^{+}} \leqslant 2 K_{2}\right\}
$$

é absorvente para o semigrupo $T(t): \mathcal{K}_{d}^{+} \rightarrow \mathcal{K}_{d}^{+}, t \geqslant 0$, isto é, para todo conjunto $B \subset \mathcal{K}_{d}^{+}$ (limitado na norma de $\mathcal{F}_{b, d}^{+}$) existe $t_{1}=t_{1}(B)>0$ tal que $T(t) B \subseteq P$, para $t \geqslant t_{1}$. De fato, se $\|u(0)\|_{H_{d}^{1}(\Omega)}<R$, então basta tomarmos $t_{1}=\ln \frac{K_{1} R^{2}}{K_{2}}$.

E do Teorema 1.3.10 temos que o atrator de trajetórias do semigrupo $\{T(t)\}$ restrito ao espaço $\mathcal{K}_{d}^{+}$é dado por

$$
\mathfrak{A}_{d}=\omega(P)=\cap_{\tau \geqslant 0}{\overline{\cup_{\theta \geqslant \tau} T(\theta) P}}^{\Theta_{l o c, d}^{+}} .
$$


Consideremos agora a equação

$$
\frac{\partial u}{\partial t}=d \Delta u-f(u)+|u|^{\alpha-1} u \quad(t, x) \in(-\infty, \infty) \times \Omega .
$$

Dizemos que uma função $u \in L_{l o c}^{\infty}\left(\mathbb{R} ; H_{d}^{1}(\Omega)\right) \cap L_{l o c}^{p}\left(\mathbb{R} ; L^{p}(\Omega)\right)$ é uma solução fraca completa da equação (3.49) se verifica a igualdade (3.49) no sentido de distribuição do espaço $\mathcal{D}^{\prime}\left(\mathbb{R}, H^{-r}(\Omega)\right)$. Observemos que $\partial_{t} u \in L_{\text {loc }}^{q}\left(\mathbb{R} ; H^{-r}(\Omega)\right)$.

Sejam $p>1$ e $E$ um espaço de Banach. Consideramos o espaço de Banach $L_{b}^{p}(\mathbb{R} ; E)$ munido da norma

$$
\|v\|_{L_{b}^{p}(\mathbb{R} ; E)}^{p}=\sup _{t \in \mathbb{R}} \int_{t}^{t+1}\|v(s)\|_{E}^{p} d s .
$$

Definamos os espaços

$\mathcal{F}_{l o c, d}=\left\{u: u \in L_{l o c}^{\infty}\left(\mathbb{R} ; H_{d}^{1}(\Omega)\right) \cap L_{l o c}^{p}\left(\mathbb{R} ; L^{p}(\Omega)\right)\right.$ e $\partial_{t} v \in L_{l o c}^{q}\left(\mathbb{R} ; H^{-r}(\Omega)\right)$, para todo $\left.T>0\right\} ;$

$$
\mathcal{K}_{d}=\left\{u \in \mathcal{F}_{l o c, d}: u \in L^{\infty}\left(-T, T ; H_{d}^{1}(\Omega)\right) \cap L^{p}\left(-T, T ; L^{p}(\Omega)\right)\right. \text { é solução fraca de }
$$

(3.49) em $[-T, T]$, para todo $T>0\}$;

$$
\begin{aligned}
\mathcal{F}_{b, d}= & \left\{u \in \mathcal{F}_{l o c, d}: u \in L^{\infty}\left(\mathbb{R} ; H_{d}^{1}(\Omega)\right) \cap L_{b}^{p}\left(\mathbb{R} ; L^{p}(\Omega)\right) \text { e } \partial_{t} u \in L_{b}^{q}\left(\mathbb{R} ; H^{-r}(\Omega)\right)\right. \\
& \left.\operatorname{com}\|u\|_{\mathcal{F}_{b, d}}<+\infty\right\}
\end{aligned}
$$

onde

$$
\|u\|_{\mathcal{F}_{b, d}} \doteq\|u\|_{L^{\infty}\left(\mathbb{R} ; H_{d}^{1}(\Omega)\right)}+\mid u\left\|_{L_{b}^{p}\left(\mathbb{R} ; L^{p}(\Omega)\right)}+\right\| \partial_{t} u \|_{L_{b}^{q}\left(\mathbb{R} ; H^{-r}(\Omega)\right)} .
$$

Além disso, assumimos que o espaço $\mathcal{F}_{l o c, d}$ é dotado da topologia $\Theta_{l o c, d}$ caracterizada em termos de sequências por: $\left\{u_{m}\right\} \subset \mathcal{F}_{l o c, d}$ converge para uma função $u \in \mathcal{F}_{l o c, d}$ se para todo intervalo $[-T, T] \subset \mathbb{R}$ temos

(i) $u_{m} \stackrel{*}{\rightarrow} u$ em $L^{\infty}\left(-T, T ; H_{d}^{1}(\Omega)\right)$,

(ii) $u_{m} \rightarrow u$ em $L^{p}\left(-T, T ; L^{p}(\Omega)\right) \mathrm{e}$

(iii) $\partial_{t} u_{m} \rightarrow \partial_{t} u$ em $L^{q}\left(-T, T ; H^{-r}(\Omega)\right)$.

Definição 3.1.8. O conjunto $\mathcal{K}_{d}$ constituído de todas as soluções fracas completas de (3.49) com norma $\|\cdot\|_{\mathcal{F}_{b, d}}<+\infty$ é denominado núcleo da equação (3.10).

Da Definição 3.1 .8 e do fato da equação (3.49) ser autônoma temos que $T(t) \mathcal{K}_{d} \subset \mathcal{K}_{d}$, para todo $t \in \mathbb{R}$. 
3 Atrator de trajetórias de uma equação parabólica

Teorema 3.1.9. Se $\mathfrak{A}_{d}$ é o atrator de trajetórias da equação (3.10) (Proposição 3.1.7) e $\mathcal{K}_{d}$ é o núcleo de (3.10), então

$$
\mathfrak{A}_{d}=\Pi_{+} \mathcal{K}_{d}
$$

onde $\Pi_{+}$denota o operador restrição ao intervalo $[0,+\infty)$. Além disso, $\mathcal{K}_{d} \subset \mathcal{F}_{b, d}$ e $\mathcal{K}_{d}$ é compacto na topologia $\Theta_{l o c, d}$.

Demonstração: Seja $u \in \mathcal{K}_{d}$ e considere $B=\left\{\Pi_{+} T(t) u: t \geqslant 0\right\}$. É claro que $B \subset \mathcal{K}_{d}^{+}$e

$$
T(t) B=B
$$

para todo $t \geqslant 0$, isto é, $B$ é estritamente invariante com respeito ao semigrupo $\{T(t): t \geqslant 0\}$. Além disso, o conjunto $B$ é limitado na norma de $\mathcal{F}_{b, d}^{+}$, pois

$$
\left\|\Pi_{+} T(t) u\right\|_{\mathcal{F}_{b, d}^{+}} \leqslant\|T(t) u\|_{\mathcal{F}_{b, d}} \equiv\|u\|_{\mathcal{F}_{b, d}}
$$

para todo $t \in \mathbb{R}$. Logo, $T(t) B \subset \mathcal{O}\left(\mathfrak{A}_{d}\right)$ quando $t \rightarrow+\infty$, onde $\mathcal{O}$ é uma vizinhança de $\mathfrak{A}_{d}$ na topologia $\Theta_{l o c, d}^{+}$. De (3.50) e do fato de $\mathfrak{A}_{d}$ ser compacto em $\Theta_{l o c, d}^{+}$segue que $B \subset \mathfrak{A}_{d}$. Em particular, $\Pi_{+} u \in \mathfrak{A}_{d}$. Portanto, $\Pi_{+} \mathcal{K}_{d} \subset \mathfrak{A}_{d}$.

Reciprocamente, tome $u_{0} \in \mathfrak{A}_{d}$. Por definição, $u_{0}$ é uma função definida no intervalo $[0,+\infty)$. Como $\mathfrak{A}_{d}$ é estritamente invariante pelo semigrupo translação $\{T(t): t \geqslant 0\}$ temos que existe uma função $u_{-1} \in \mathfrak{A}_{d}$ tal que $T(1) u_{-1}=u_{0}$. Definamos $u(s) \doteq u_{-1}(s+1)$, para $s \geqslant-1$. Logo, $u(s) \equiv u_{0}(s)$ para $s \geqslant 0$ e $u(s)$ é uma solução fraca de (3.10) para $s \geqslant-1$. Repetindo esse processo indefinidamente encontramos uma função $u(s)$ definida para $s \in \mathbb{R}$, isto é, esta é uma solução fraca completa da equação (3.10) tal que $u(s) \equiv u_{0}(s)$ para $s \geqslant 0$. Além disso, da forma como construímos a função $u$ resulta que

$$
\|u\|_{\mathcal{F}_{b, d}} \leqslant\left\|\mathfrak{A}_{d}\right\|_{\mathcal{F}_{b, d}^{+}}
$$

Portanto, $u$ pertence ao núcleo $\mathcal{K}_{d}$ e $\Pi_{+} u=u_{0}$. Assim, $\mathfrak{A}_{d} \subset \Pi_{+} \mathcal{K}_{d}$.

Observação 3.1.3. No artigo de Chepyzhov, V. V., Vishik, M. I. and Zelik, S. V. [14], os autores denominam o atrator obtido por meio do Teorema 3.1.7 de atrator de trajetórias fraco da equação (3.10). Se substituirmos a condição de atração fraca pela condição de atração forte, então podemos afirmar, em virtude do resultado demonstrado pelos autores, que o atrator de trajetórias obtido pelo Teorema 3.1.7 é o atrator de trajetórias forte do semigrupo translação $\{T(t)\}$ atuando sobre o espaço de trajetórias $\mathcal{K}_{d}^{+}$do problema (3.10). 


\subsection{Semicontinuidade superior de uma família de atra- tores de trajetórias}

Nessa seção estamos interessados em estudar o comportamento assintótico da família de atratores de trajetórias do problema (3.8) quando o parâmetro $\epsilon \rightarrow 0^{+}$. Se $\left\{\mathfrak{A}_{\epsilon}: \epsilon \in\right.$ $\left.\left[0, \epsilon_{0}\right]\right\}$ é a família de atratores de trajetórias de (3.8), então o teorema principal dessa seção demonstra que essa família é semicontínua superiormente em $\epsilon=0$. Para atingirmos o nosso objetivo é fundamental que as soluções satisfaçam estimativas uniformes em relação a $\epsilon$.

A principal referência para essa seção é o livro de Chepyzhov, V. V.; Vishik, M. I. [9], mas, como já observamos anteriormente, os autores consideraram apenas problemas com condição de Dirichlet, embora as contas sejam parecidas.

Vamos considerar o problema

$$
\left\{\begin{array}{l}
\frac{\partial u}{\partial t}=d \Delta u-f(u)+\epsilon|u|^{\alpha-1} u \quad(t, x) \in(0, \infty) \times \Omega \\
\frac{\partial u}{\partial \nu}=0 \quad(t, x) \in(0, \infty) \times \Gamma \\
u(0, x)=u_{0}(x) \quad x \in \Omega,
\end{array}\right.
$$

onde $\Omega \subset \mathbb{R}^{n}$ é um subconjunto aberto, limitado, conexo, com fronteira suave $\Gamma$ e para cada ponto $x \in \Gamma, \nu=\nu(x)$ indica o vetor normal exterior unitário a $\Gamma$ no ponto $x$. Além disso, $d$ está fixado e verifica as condições dadas na Proposição 3.1.7, $\alpha \in(0,1), \epsilon \in\left[0, \epsilon_{0}\right], \epsilon_{0}<1$ e $f: \mathbb{R} \rightarrow \mathbb{R}$ é uma função de classe $C^{1}$ satisfazendo as hipóteses (3.11)-(3.13).

Pelo Teorema 3.1.2 existe pelo menos uma solução fraca global para a equação (3.51) com dado inicial $u_{0} \in L^{2}(\Omega)$. Mas lembremos que estamos considerando $u_{0} \in H_{d}^{1}(\Omega)$ o que nos permite obter uma estimativa a priori adicional. Os espaços de trajetórias de (3.51) quando $\epsilon \in\left(0, \epsilon_{0}\right]$ e $\epsilon=0$ são dados, respectivamente, por

$$
\begin{aligned}
\mathcal{K}_{\epsilon}^{+}= & \left\{u_{\epsilon}: u_{\epsilon} \in L^{\infty}\left(0, T ; H_{d}^{1}(\Omega)\right) \cap L^{p}\left(0, T ; L^{p}(\Omega)\right) \text { é solução fraca de }(3.51)\right. \\
& \text { para todo } T>0\} \\
\mathcal{K}_{0}^{+}= & \left\{u: u \in L^{\infty}\left(0, T ; H_{d}^{1}(\Omega)\right) \cap L^{p}\left(0, T ; L^{p}(\Omega)\right) \text { é solução fraca de }(3.51)\right. \\
& \text { com } \epsilon=0 \text { para todo } T>0\} .
\end{aligned}
$$

Consideremos os conjuntos $\mathcal{F}_{l o c}^{+}, \mathcal{F}_{b}^{+}$e a topologia $\Theta_{l o c}^{+}$, onde $\mathcal{F}_{l o c}^{+}$munido da topologia $\Theta_{l o c}^{+}$é um espaço topológico e $\mathcal{F}_{b}^{+} \subset \mathcal{F}_{\text {loc }}^{+}$munido da norma $\|\cdot\|_{\mathcal{F}_{b}^{+}}$é um espaço de Banach. As definições desses espaços e da topologia são dadas como na Seção 3.1 e por isso omitimos os detalhes. Observemos que esses espaços e a topologia não mudam quando $\epsilon$ varia no intervalo $\left[0, \epsilon_{0}\right]$. 
Proposição 3.2.1. O problema (3.51) com $\epsilon>0$ possui um atrator de trajetórias $\mathfrak{A}_{\epsilon}$.

Demonstração: Vamos verificar que as soluções $u_{\epsilon}$ satisfazem estimativas uniformes em relação ao parâmetro $\epsilon$. Para não carregar a notação denotaremos $u \doteq u_{\epsilon}$.

Se $u$ é uma solução fraca de (3.51), então do fato que $\epsilon \leqslant \epsilon_{0}$, decorre que

$$
\frac{d}{d t} \int_{\Omega}|u(s)|^{2} d x+2\|u(s)\|_{H_{d}^{1}(\Omega)}^{2}+c_{1}(1-\epsilon) \int_{\Omega}|u(s)|^{p} d x \leqslant C_{3}
$$

onde

$$
C_{3}=2|\Omega|\left[c_{3}+\left(\frac{4}{c_{1} p}\right)^{\frac{2}{p-2}}\left(\frac{p-2}{p}\right)+\epsilon_{0}\left(\frac{2(\alpha+1)}{c_{1} p}\right)^{\frac{\alpha+1}{p-(\alpha+1)}}\left(\frac{p-(\alpha+1)}{p}\right)\right] .
$$

Como fizemos no Corolário 3.1.4, item $(i)$, obtemos

$$
\|u(s)\|_{L^{2}(\Omega)}^{2} \leqslant\|u(0)\|_{L^{2}(\Omega)}^{2} e^{-2 s}+\frac{C_{3}}{2} .
$$

Logo,

$$
\|T(t) u\|_{L^{\infty}\left(\mathbb{R}_{+}, L^{2}(\Omega)\right)}^{2} \leqslant\|u(0)\|_{L^{2}(\Omega)}^{2} e^{-2 t}+\frac{C_{3}}{2},
$$

para $t \geqslant 0$.

Integrando (3.52) no intervalo $[t, t+1]$ como fizemos no Corolário 3.1.4, item $(i i)$, ficamos com

$$
2 \int_{t}^{t+1}\|u(s)\|_{H_{d}^{1}(\Omega)}^{2} d s+c_{1}(1-\epsilon) \int_{t}^{t+1}\|u(s)\|_{L^{p}(\Omega)}^{p} d s \leqslant\|u(0)\|_{L^{2}(\Omega)}^{2} e^{-2 t}+\frac{3 C_{3}}{2},
$$

donde

$$
2\|T(t) u\|_{L^{2}\left(0,1 ; H_{d}^{1}(\Omega)\right)}^{2}+c_{1}(1-\epsilon)\|T(t) u\|_{L^{p}\left(0,1 ; L^{p}(\Omega)\right)}^{p} \leqslant\|u(0)\|_{L^{2}(\Omega)}^{2} e^{-2 t}+\frac{3 C_{3}}{2},
$$

para $t \geqslant 0$.

Como fizemos em (3.41), temos que

$$
\|T(t) u\|_{L^{\infty}\left(\mathbb{R}_{+}, H_{d}^{1}(\Omega)\right)}^{2} \leqslant\|u(0)\|_{H_{d}^{1}(\Omega)}^{2} e^{-t}+\max \left\{\frac{C_{3}}{2}, C_{3} \alpha \epsilon_{0}^{2}+(1-\alpha)|\Omega| \epsilon_{0}^{2}\right\},
$$

para $t \geqslant 0$. 
E analogamente a (3.45), obtemos

$$
\begin{aligned}
& \left\|\partial_{t} T(t) u\right\|_{L^{q}\left(0,1 ; H^{-r}(\Omega)\right)} \leqslant k_{1}\left(\sqrt{\frac{3 C_{1}}{4}}+\sqrt{\frac{1}{2}}\|u(0)\|_{L^{2}(\Omega)} e^{-t}\right)+ \\
& +k_{2} c_{2}^{\frac{1}{q}}\left(\frac{1}{c_{1}}\left[\|u(0)\|_{L^{2}(\Omega)}^{2} e^{-2 t}+\frac{3 C_{1}}{2}\right]+|\Omega|+1\right)+ \\
& +k_{3} \epsilon_{0}\left(\frac{\alpha q}{4}\|u(0)\|_{L^{2}(\Omega)}^{2} e^{-2 t}+\frac{\alpha q C_{1}}{4}+\frac{2-\alpha q}{2}|\Omega|+1\right),
\end{aligned}
$$

onde $r=\max \left\{1, n\left(\frac{1}{q}-\frac{1}{2}\right)\right\}$ e $t \geqslant 0$.

De (3.57), obtemos constantes positivas $\tilde{K}_{1}, \tilde{K}_{2}$ tais que

$$
\left\|\partial_{t} T(t) u\right\|_{\left.L^{q}\left(0,1 ; H^{-r}(\Omega)\right)\right)} \leqslant \tilde{K}_{1}\|u(0)\|_{L^{2}(\Omega)}^{2} e^{-t}+\tilde{K}_{2}
$$

para $t \geqslant 0$, onde

$$
\tilde{K}_{1}=k_{1} \sqrt{\frac{1}{2}}+\frac{k_{2} c_{2}^{\frac{1}{q}}}{c_{1}}+\frac{k_{3} \epsilon_{0} \alpha q}{4}
$$

e

$$
\tilde{K}_{2}=k_{1} \sqrt{\frac{3 C_{1}}{4}}+\frac{k_{2} c_{2}^{\frac{1}{q}}}{c_{1}} \frac{3 C_{1}}{2}+k_{2} c_{2}^{\frac{1}{q}}(|\Omega|+1)+k_{3} \epsilon_{0}\left(\frac{\alpha q C_{1}}{4}+\frac{2-\alpha q}{2}|\Omega|+1\right)+k_{1} \sqrt{\frac{1}{2}}
$$

Concluímos de (3.55), (3.56) e (3.58) que existem constantes positivas $K_{1}$ e $K_{2}$, independentes de $\epsilon$, tais que

$$
\|T(\tau) u\|_{\mathcal{F}_{b}^{+}} \leqslant K_{1}\|u(0)\|_{H_{d}^{1}(\Omega)}^{2} e^{-t}+K_{2}
$$

para todo $\tau \geqslant 0$, onde

$$
K_{1}=\max \left\{1, \frac{1}{c_{1}\left(1-\epsilon_{0}\right)}, \tilde{K}_{1}\right\}
$$

e

$$
K_{2}=\max \left\{\frac{3 C_{3}}{2\left(1-\epsilon_{0}\right) c_{1}}+1, \max \left\{\frac{C_{3}}{2}, C_{3} \alpha \epsilon_{0}^{2}+(1-\alpha)|\Omega| \epsilon_{0}^{2}\right\}+1, \tilde{K}_{2}\right\} .
$$

É claro de (3.59) que $\mathcal{K}_{\epsilon}^{+} \subset \mathcal{F}_{b}^{+}$para $\epsilon>0$. 
Da desigualdade (3.59) segue que o conjunto

$$
P=\left\{u \in \mathcal{K}_{\epsilon}^{+}:\|u\|_{\mathcal{F}_{b}^{+}} \leqslant 2 K_{2}\right\}
$$

é absorvente para o semigrupo $T(t): \mathcal{K}_{\epsilon}^{+} \rightarrow \mathcal{K}_{\epsilon}^{+}, t \geqslant 0$.

E do Teorema 1.3.10 temos que o atrator de trajetórias do semigrupo $\{T(t)\}$ restrito ao espaço $\mathcal{K}_{\epsilon}^{+}$é dado por

$$
\mathfrak{A}_{\epsilon}=\omega(P)=\cap_{\tau \geqslant 0}{\overline{\cup_{\theta \geqslant \tau} T(\theta) P}}^{\Theta_{l o c}^{+}} .
$$

Observação 3.2.1. Decorre da demonstração da Proposição 3.2.1 que o conjunto $P$ definido em (3.60) é um conjunto absorvente para o semigrupo $T(t): \mathcal{K}_{\epsilon}^{+} \rightarrow \mathcal{K}_{\epsilon}^{+}$para todo $\epsilon \in\left(0, \epsilon_{0}\right]$, pois a constante $K_{2}$ independe de $\epsilon$.

Proposição 3.2.2. O problema (3.51) com $\epsilon=0$ possui um atrator de trajetórias $\mathfrak{A}_{0}$.

Demonstração: A demonstração é idêntica àquela que fizemos na Proposição 3.1.7, por isso vamos omitir as contas.

Observação 3.2.2. Da Proposição 3.1 .9 resulta que $\mathfrak{A}_{\epsilon}=\Pi_{+} \mathcal{K}_{\epsilon}$, onde $\mathcal{K}_{\epsilon}$ é o núcleo da equação (3.51) com $\epsilon \in\left[0, \epsilon_{0}\right]$.

Proposição 3.2.3. Se $\left\{u_{\epsilon_{m}}^{m}\right\} \subset \mathcal{K}_{\epsilon_{m}}^{+} e\left\{\epsilon_{m}\right\} \subset\left(0, \epsilon_{0}\right]$ são sequências com $\epsilon_{m} \rightarrow 0^{+}$quando $m \rightarrow+\infty$, então $u_{\epsilon_{m}}^{m} \rightarrow \tilde{u}$ na topologia $\Theta_{\text {loc }}^{+}$e $\tilde{u} \in \mathcal{K}_{0}^{+}$.

Demonstração: As funções $u_{\epsilon_{m}}^{m} \in \mathcal{K}_{\epsilon_{m}}^{+}$satisfazem a equação

$$
\frac{\partial u_{\epsilon_{m}}^{m}}{\partial t}=d \Delta u_{\epsilon_{m}}^{m}-f\left(u_{\epsilon_{m}}^{m}\right)+\epsilon_{m}\left|u_{\epsilon_{m}}^{m}\right|^{\alpha-1} u_{\epsilon_{m}}^{m} .
$$

Da inclusão $\mathcal{K}_{\epsilon_{m}}^{+} \subset \mathcal{F}_{b}^{+}$resulta que $\left\|u_{\epsilon_{m}}^{m}\right\|_{\mathcal{F}_{b}^{+}}<+\infty$, ou seja, a sequência $\left\{u_{\epsilon_{m}}^{m}\right\}$ é pré-compacta na topologia $\Theta_{l o c}^{+}$. Logo, existe uma subsequência indicada por $u_{\epsilon_{m}}^{m}$ tal que $u_{\epsilon_{m}}^{m} \rightarrow \tilde{u}$ em $\Theta_{l o c}^{+}$para alguma função $\tilde{u} \in \mathcal{F}_{l o c}^{+}$, isto é,

(i) $u_{\epsilon_{m}}^{m} \rightarrow \tilde{u}$ em $L^{2}\left(0, T ; H_{d}^{1}(\Omega)\right)$;

(ii) $u_{\epsilon_{m}}^{m} \rightarrow \tilde{u}$ em $L^{p}\left(0, T ; L^{p}(\Omega)\right)$;

(iii) $u_{\epsilon_{m}}^{m} \stackrel{*}{\rightarrow} \tilde{u}$ em $L^{\infty}\left(0, T ; L^{2}(\Omega)\right)$;

(iv) $\partial_{t} u_{\epsilon_{m}}^{m} \rightarrow \partial_{t} \tilde{u}$ em $L^{q}\left(0, T ; H^{-r}(\Omega)\right)$.

Como observado na demonstração do Teorema 3.1.2, temos também as seguintes convergências 
(v) $\Delta u_{\epsilon_{m}}^{m} \rightarrow \Delta \tilde{u}$ em $L^{2}\left(0, T ; H_{d}^{-1}(\Omega)\right)$;

(vi) $f\left(u_{\epsilon_{m}}^{m}\right)-\epsilon_{m}\left|u_{\epsilon_{m}}^{m}\right|^{\alpha-1} u_{\epsilon_{m}}^{m} \rightarrow f(\tilde{u})-\epsilon_{m}|\tilde{u}|^{\alpha-1} \tilde{u} \operatorname{em~} L^{q}\left(0, T ; L^{q}(\Omega)\right)$;

(vii) $\frac{\partial u_{\epsilon_{m}}^{m}}{\partial \nu} \rightarrow \frac{\partial \tilde{u}}{\partial \nu}$ em $L^{2}\left(0, T ; H^{-\frac{1}{2}}(\Gamma)\right)$.

De 3.17 decorre que $\epsilon_{m}\left|u_{\epsilon_{m}}^{m}\right|^{\alpha-1} u_{\epsilon_{m}}^{m} \rightarrow 0$ em $L^{q}\left(0, T ; H^{-r}(\Omega)\right)$ quando $m \rightarrow+\infty$, onde $r=\max \left\{1, n\left(\frac{1}{q}-\frac{1}{2}\right)\right\}$.

Portanto, tomando $m \rightarrow \infty$ em (3.62) obtemos que $\tilde{u}$ verifica

$$
\frac{\partial \tilde{u}}{\partial t}=d \Delta \tilde{u}-f(\tilde{u})
$$

no sentido de distribuição do espaço $\mathcal{D}^{\prime}\left(0, T ; H^{-r}(\Omega)\right)$ para todo $T>0$. Além disso, $\|\tilde{u}\|_{\mathcal{F}_{b}^{+}}<$ $+\infty$, ou seja, $\tilde{u} \in \mathcal{K}_{0}^{+}$.

Vamos enunciar o resultado principal dessa subseção.

Teorema 3.2.4. Se $\mathfrak{A}_{\epsilon}$ é o atrator de trajetórias do problema (3.51) com $\epsilon \in\left[0, \epsilon_{0}\right]$, então a família de atratores $\left\{\mathfrak{A}_{\epsilon}: \epsilon \in\left[0, \epsilon_{0}\right]\right\}$ é semicontínua superiormente em $\epsilon=0$.

Demonstração: Suponhamos que a tese do enunciado é falsa. Então existem uma vizinhança $\mathcal{O}$ de $\mathfrak{A}_{0}$ na topologia $\Theta_{l o c}^{+}$e uma sequência $\epsilon_{m} \rightarrow 0^{+}$satisfazendo

$$
\mathfrak{A}_{\epsilon_{m}} \not \subset \mathcal{O}\left(\mathfrak{A}_{0}\right)=\mathcal{O}\left(\Pi_{+} \mathcal{K}_{0}\right) \text {. }
$$

Da invariância estrita do atrator de trajetórias $\mathfrak{A}_{\epsilon_{m}}$ com respeito ao semigrupo translação $\{T(t): t \geqslant 0\}$, existe sequência $\tau_{m} \rightarrow+\infty$ tal que $T\left(\tau_{m}\right) \mathfrak{A}_{\epsilon_{m}} \not \subset \mathcal{O}\left(\Pi_{+} \mathcal{K}_{0}\right)$. Logo, existe uma solução $u_{\epsilon_{m}} \in \mathfrak{A}_{\epsilon_{m}}$ satisfazendo

$$
\widetilde{u_{\epsilon_{m}}} \doteq T\left(\tau_{m}\right) u_{\epsilon_{m}}=u_{\epsilon_{m}}\left(\tau_{m}+\cdot\right) \notin \mathcal{O}\left(\Pi_{+} \mathcal{K}_{0}\right)
$$

Observemos que $\widetilde{u_{\epsilon_{m}}}$ é uma solução da equação (3.51) com $\epsilon=\epsilon_{m}$ para $t \geqslant-\tau_{m}$ e $\widetilde{u_{\epsilon_{m}}}(t)=u_{\epsilon_{m}}(t)$ para $t \geqslant 0$.

Na Proposição 3.2.1 vimos que as soluções do problema (3.51) com $\epsilon \in\left(0, \epsilon_{0}\right]$ verificam a estimativa (3.60) uniforme em relação a $\epsilon$. Portanto, podemos escrever

$$
\begin{aligned}
& \left(\sup _{t \geqslant-\tau_{m}} \int_{t}^{t+1}\left\|\widetilde{u_{\epsilon_{m}}}(s)\right\|_{L^{p}(\Omega)}^{p} d s\right)^{\frac{1}{p}}+\operatorname{ess} \sup _{t \geqslant-\tau_{m}}\left\|\widetilde{u_{\epsilon_{m}}}(s)\right\|_{H_{d}^{1}(\Omega)}+ \\
& \left(\sup _{t \geqslant-\tau_{m}} \int_{t}^{t+1}\left\|\partial_{t} \widetilde{u_{\epsilon_{m}}}(s)\right\|_{H^{-r}(\Omega)}^{q} d s\right)^{\frac{1}{q}} \leqslant 2 K_{2},
\end{aligned}
$$

onde $K_{2}$ é, como já sabemos, independente de $\epsilon$. 
3 Atrator de trajetórias de uma equação parabólica

Da desigualdade (3.65) resulta que $\left\{\widetilde{u_{\epsilon_{m}}}(t)\right\}$ é pré-compacta em relação a topologia $\Theta(-T, T)$ definida sobre o espaço

$$
\mathcal{F}(-T, T)=\left\{v: v \in L^{p}\left(-T, T ; L^{p}(\Omega)\right) \cap L^{\infty}\left(-T, T ; H_{d}^{1}(\Omega)\right) \text { e } \partial_{t} v \in L^{q}\left(-T, T ; H^{-r}(\Omega)\right)\right\},
$$

para todo $T>0$ e quando $\tau_{m} \geqslant T$. Então podemos extrair uma subsequência $\left\{\epsilon_{m^{\prime}}\right\} \subseteq\left\{\epsilon_{m}\right\}$ tal que $\left\{\widetilde{u_{\epsilon_{m}^{\prime}}}\right\}$ converge na topologia $\Theta(-T, T)$. E como isso vale para todo $T>0$ fixo, podemos, pelo argumento da diagonal de Cantor ou método diagonal, construir uma subsequência $\left\{\epsilon_{m^{\prime \prime}}\right\} \subseteq\left\{\epsilon_{m}\right\}$ tal que

$$
\widetilde{u_{\epsilon_{m^{\prime \prime}}}} \rightarrow \tilde{u}
$$

quando $m^{\prime \prime} \rightarrow+\infty$, em $\Theta(-T, T)$ para todo $T>0$. Assim $\widetilde{u_{\epsilon_{m^{\prime \prime}}}} \rightarrow \tilde{u}$ em $\Theta_{l o c}^{+}$.

De (3.66), obtemos a seguinte estimativa para a função limite $\tilde{u}$

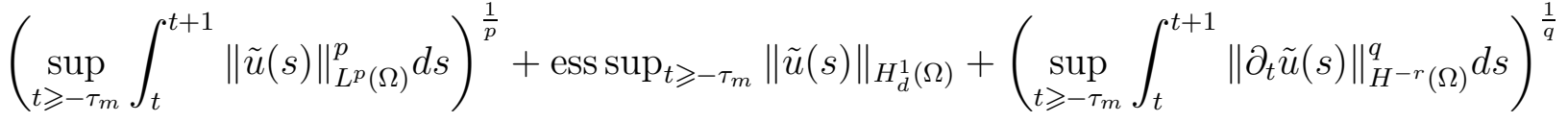

$$
\begin{aligned}
& \leqslant 2 K_{2} \text {. }
\end{aligned}
$$

De (3.67) temos, em particular, que

$$
\tilde{u} \in \mathcal{F}_{b}=\left\{v: v \in L_{b}^{p}\left(\mathbb{R}, L^{p}(\Omega)\right) \cap L_{b}^{2}\left(\mathbb{R}, H_{d}^{1}(\Omega)\right) \cap L^{\infty}\left(\mathbb{R}, L^{2}(\Omega)\right) \text { e } \partial_{t} v \in L_{b}^{q}\left(\mathbb{R}, H^{-r}(\Omega)\right)\right\}
$$

Afirmamos que $\tilde{u} \in \mathcal{K}_{0}$. De fato, como a equação (3.51) é autônoma podemos aplicar a Proposição 3.2.3 para a sequência $\left\{\widetilde{u_{\epsilon_{m^{\prime \prime}}}}\right\}$ contida no espaço das soluções de (3.51) definidas no intervalo $[-T,+\infty)$ e que é convergente na topologia $\Theta(-T, T)$. Portanto, ú é uma solução fraca da equação (3.63) no intervalo $[-T,+\infty), \forall T>0$, e de (3.67) temos que $\tilde{u}$ é limitado na norma do espaço $\mathcal{F}_{b}$, ou seja, $\tilde{u} \in \mathcal{K}_{0}$.

De (3.66) e como $\tilde{u} \in \mathcal{K}_{0}$ concluímos que $\Pi_{+} \tilde{u} \in \mathfrak{A}_{0}$ e $\Pi_{+} \widetilde{u_{\epsilon_{m^{\prime \prime}}}} \rightarrow \Pi_{+} \tilde{u}$ em $\Theta_{+}^{\text {loc }}$ quando $m^{\prime \prime} \rightarrow+\infty$, ou seja, para $m^{\prime \prime}$ suficientemente grande temos que $\Pi_{+} \widetilde{u_{\epsilon_{m^{\prime \prime}}}} \in \mathcal{O}\left(\mathfrak{A}_{0}\right)$. Mas isso contradiz (3.64).

Do Teorema 3.2.4 decorre o próximo resultado

Corolário 3.2.5. Se $\mathcal{K}_{\epsilon}$ e $\mathcal{K}_{0}$ são os núcleos de (3.51) e (3.63), respectivamente, então

$$
\mathcal{K}_{\epsilon} \subset \mathcal{O}\left(\mathcal{K}_{0}\right)
$$

quando $\epsilon \rightarrow 0^{+}$, onde $\mathcal{O}$ é uma vizinhança de $\mathcal{K}_{0}$ na topologia $\Theta_{\text {loc }}$. 


\subsection{Difusão grande}

Nessa seção vamos examinar a questão da continuidade do atrator de trajetórias da equação (3.3) com relação ao coeficiente de difusão $d$. Como observamos no início desse capítulo há vários trabalhos que tratam desse assunto para equações cujo problema de Cauchy correspondente goza da propriedade de unicidade de solução. A nossa investigação vai se restringir ao seguinte problema

$$
\left\{\begin{array}{l}
\frac{\partial u}{\partial t}=d \Delta u-f(u)+|u|^{\alpha-1} u \quad(t, x) \in(0, \infty) \times \Omega \\
\frac{\partial u}{\partial \nu}=0 \quad(t, x) \in(0, \infty) \times \Gamma
\end{array}\right.
$$

onde $\Omega \subset \mathbb{R}^{n}(n \geqslant 3)$ é um subconjunto aberto, limitado, conexo, com fronteira suave $\Gamma \mathrm{e}$ para cada ponto $x \in \Gamma, \nu=\nu(x)$ indica o vetor normal exterior unitário a $\Gamma$ no ponto $x$. Além disso, $d$ verifica as condições dadas na Proposição 3.1.7, $\alpha \in(0,1)$ e $f: \mathbb{R} \rightarrow \mathbb{R}$ é uma função de classe $C^{1}$ satisfazendo as seguintes hipóteses: existem constantes positivas $c_{f}, C$, $c_{1}, c_{2}, c_{3}$ e $2<p<\frac{2 n}{n-2}$ tais que

$$
\begin{gathered}
f^{\prime}(u) \geqslant c_{f}, \\
\left|f\left(u_{1}\right)-f\left(u_{2}\right)\right| \leqslant C\left|u_{1}-u_{2}\right|\left(1+\left|u_{1}\right|^{p-2}+\left|u_{2}\right|^{p-2}\right), \\
c_{1}|u|^{p}-c_{3} \leqslant f(u) \cdot u
\end{gathered}
$$

e

$$
|f(u)|^{\frac{p}{p-1}} \leqslant c_{2}\left(|u|^{p}+1\right),
$$

para quaisquer $u, u_{1}, u_{2} \in \mathbb{R}$.

Sabemos que se tomarmos o dado inicial $u_{0} \in L^{2}(\Omega)$, então pelo Teorema 3.1.2 o problema (3.68) admite pelo menos uma solução fraca global. Além disso, se $u_{0} \in H_{d}^{1}(\Omega)$, então é possível obter a estimativa a priori (3.35). A Proposição 3.1 .7 garante a existência de um atrator de trajetórias $\mathfrak{A}_{d}$ para o problema (3.68) para cada $d>1$ tal que $d \lambda_{1}>2$ e temos que a família de atratores $\left\{\mathfrak{A}_{d}\right\}_{d}$ está contida no conjunto $P$ definido em (3.47). Observemos que $P$ é uma bola no espaço de Banach $\mathcal{F}_{b, d}^{+}$, cujo raio independe de $d$.

Sejam $\left\{\lambda_{j}\right\}_{j=0}^{\infty}$ e $\left\{w_{j}\right\}_{j=0}^{\infty}$ os autovalores e as autofunções normalizadas, respectivamente, do problema de Neumann

$$
\left\{\begin{array}{l}
\Delta w+\lambda w=0 \quad \text { em } \Omega \\
\left.\frac{\partial w}{\partial \nu}\right|_{\Gamma}=0
\end{array}\right.
$$


Do Teorema 1.1.29 sabemos que $\lambda_{0}=0<\lambda_{1}<\lambda_{2}<\ldots<\lambda_{j} \rightarrow \infty,\left\{w_{j}\right\}_{j \in \mathbb{N}}$ é um sistema ortonormal completo para $L^{2}(\Omega)$ e $w_{0}(x)=\frac{1}{\sqrt{|\Omega|}}$.

Consideremos a decomposição ortogonal de $L^{2}(\Omega)=\left[w_{0}\right] \oplus Y$, onde

$$
Y=\left[w_{2}, w_{3}, \ldots\right]=\left\{v: \int_{\Omega} v(x) w_{0}(x) d x=0\right\}=\left\{v: \int_{\Omega} v(x) d x=0\right\}
$$

é o espaço das funções de $L^{2}(\Omega)$ com média nula.

Se $u=u(t, x)$ é uma solução de (3.68), então vamos adotar a decomposição

$$
u(t, x)=\beta(t) w_{0}(x)+v(x, t)
$$

escrita de maneira única, onde

$$
\begin{gathered}
\beta(t)=\int_{\Omega} u(t, x) w_{0}(x) d x=\frac{1}{\sqrt{|\Omega|}} \int_{\Omega} u(t, x) d x, \\
v(t, x)=u(t, x)-\beta(t) w_{0}(x) .
\end{gathered}
$$

A decomposição (3.73) nos permite reescrever a equação (3.68) como um sistema de equações da seguinte forma

$$
\left\{\begin{array}{l}
v_{t}=d \Delta v-f\left(\beta w_{0}+v\right)+\left|\beta w_{0}+v\right|^{\alpha-1}\left(\beta w_{0}+v\right)+\frac{1}{|\Omega|} \int_{\Omega}\left[f\left(\beta w_{0}+v\right)-\right. \\
\left.-\left|\beta w_{0}+v\right|^{\alpha-1}\left(\beta w_{0}+v\right)\right] d x \\
\beta_{t}=\frac{1}{\sqrt{|\Omega|}} \int_{\Omega}\left[-f\left(\beta w_{0}+v\right)+\left|\beta w_{0}+v\right|^{\alpha-1}\left(\beta w_{0}+v\right)\right] d x .
\end{array}\right.
$$

Queremos demonstrar que se $u(t, x)=\beta(t) w_{0}(x)+v(t, x)$ é uma solução da equação (3.68), $d \rightarrow+\infty$ e $\mathfrak{A}_{\infty}$ é o atrator de trajetórias da equação diferencial ordinária

$$
\left\{\begin{array}{l}
\gamma^{\prime}(t)=-f(\gamma(t))+|\gamma(t)|^{\alpha-1} \gamma(t), \quad t \geqslant 0 \\
\gamma(0)=\gamma_{0} \in \mathbb{R}
\end{array}\right.
$$

então

- a função componente $v$ satisfaz $v \rightarrow 0$ na topologia $\Theta_{l o c}^{+}$; e

- a família $\left\{\mathfrak{A}_{d}\right\}_{d} \cup\left\{\widetilde{\mathfrak{A}_{\infty}}\right\}$ é semicontínua superiormente em $d=\infty$, onde $\widetilde{\mathfrak{A}_{\infty}}$ é a imersão de $\mathfrak{A}_{\infty}$ em $\left[w_{0}\right] \oplus Y$ através da aplicação $\gamma \in \mathbb{R} \mapsto \gamma w_{0}$.

Vamos enunciar um lema simples. 
Lema 3.3.1. Seja $\alpha \in(0,1)$. Se $h: \mathbb{R} \rightarrow \mathbb{R}$ é a função definida por $h(x)=|x|^{\alpha-1} x$, então $\left|h\left(x_{1}\right)-h\left(x_{2}\right)\right| \leqslant 2^{1-\alpha}\left|x_{1}-x_{2}\right|^{\alpha}$, isto é, h é uma função holderiana de ordem $\alpha$.

Demonstração: Basta observar que a função contínua

$$
y(s)=\left\{\begin{array}{l}
\frac{\|\left. s\right|^{\alpha-1} s-1 \mid}{|s-1|^{\alpha}} \text { se } s \neq 1 \\
0 \quad \text { se } s=1
\end{array}\right.
$$

atinge seu valor máximo em $s=-1$ quando $y(-1)=2^{1-\alpha}$.

Agora enunciaremos o nosso resultado principal.

Teorema 3.3.2. Se $\widetilde{\mathfrak{A}_{\infty}}$ é a imersão de $\mathfrak{A}_{\infty}$ em $L^{2}(\Omega)=\left[w_{0}\right] \oplus Y$, então a família de atratores de trajetórias $\left.\left\{\mathfrak{A}_{d}\right\}_{d} \cup \widetilde{\mathfrak{A}_{\infty}}\right\}$ é semicontínua superiormente em $d=\infty$, isto é, para toda vizinhança $\mathcal{O}$ de $\widetilde{\mathfrak{A}_{\infty}}$ na topologia $\Theta_{\text {loc }}^{+}$existe $d_{0}>0$ tal que se $d \geqslant d_{0}$, então $\mathfrak{A}_{d} \subset \mathcal{O}\left(\widetilde{\mathfrak{A}_{\infty}}\right)$.

Demonstração: Tomemos $u \in \mathfrak{A}_{d}$, então podemos escrever $u=\beta w_{0}+v$. Além disso, $u$ satisfaz o seguinte sistema de equações

$$
\left\{\begin{array}{l}
v_{t}=d \Delta v-f\left(\beta w_{0}+v\right)+\left|\beta w_{0}+v\right|^{\alpha-1}\left(\beta w_{0}+v\right)+\frac{1}{|\Omega|} \int_{\Omega}\left[f\left(\beta w_{0}+v\right)-\right. \\
\left.-\left|\beta w_{0}+v\right|^{\alpha-1}\left(\beta w_{0}+v\right)\right] d x \\
\beta_{t}=G(\beta, 0)+[G(\beta, v)-G(\beta, 0)]
\end{array}\right.
$$

onde

$$
G(\beta, v)=\frac{1}{\sqrt{|\Omega|}} \int_{\Omega}\left[-f\left(\beta w_{0}+v\right)+\left|\beta w_{0}+v\right|^{\alpha-1}\left(\beta w_{0}+v\right)\right] d x
$$

Vamos começar demonstrando que $v \rightarrow 0$ em $\Theta_{l o c}^{+}$quando $d \rightarrow+\infty$, isto é, $v \stackrel{*}{\rightarrow} 0$ em $L^{\infty}\left(0, T ; H^{1}(\Omega)\right), v \rightarrow 0$ em $L^{p}\left(0, T ; L^{p}(\Omega)\right)$ e $\partial_{t} v \rightarrow 0$ em $L^{q}\left(0, T ; H^{-r}(\Omega)\right)$, para todo $T>0$.

Como $\mathfrak{A}_{d} \subset P$, onde o conjunto $P$ foi definido em (3.47), usaremos com frequência a constante $K=2 K_{2}$ como limitante superior. Observemos que essa constante independe de $d$.

Usando a Proposição 3.1.3 item (ii), a hipótese (3.71) e a Desigualdade de Poincaré 1.1.27, obtemos

$$
\begin{aligned}
& \frac{d}{d t}\|v\|_{L^{2}(\Omega)}^{2}+2 d \lambda_{1}\|v\|_{L^{2}(\Omega)}^{2}+2 c_{1} \int_{\Omega}\left|\beta w_{0}+v\right|^{p} d x \leqslant 2 c_{3}|\Omega|+2 \int_{\Omega}\left|\beta w_{0}+v\right|^{\alpha+1}+ \\
& +2 \int_{\Omega} \frac{\beta}{\sqrt{|\Omega|}}\left[f\left(\beta w_{0}+v\right)-\left|\beta w_{0}+v\right|^{\alpha-1}\left(\beta w_{0}+v\right)\right] d x .
\end{aligned}
$$


De (3.77), da hipótese (3.72) e da Desigualdade de Young (1.3), decorre que

$$
\begin{aligned}
& \frac{d}{d t}\|v\|_{L^{2}(\Omega)}^{2}+2 d \lambda_{1}\|v\|_{L^{2}(\Omega)}^{2}+2 c_{1} \int_{\Omega}\left|\beta w_{0}+v\right|^{p} d x \leqslant 2 c_{3}|\Omega|+ \\
& +\frac{c_{1}}{3} \int_{\Omega}\left|\beta w_{0}+v\right|^{p} d x+2|\Omega|\left(\frac{6(\alpha+1)}{c_{1} p}\right)^{\frac{\alpha+1}{p-(\alpha+1)}} \frac{p-(\alpha+1)}{p}+ \\
& +\frac{c_{1}}{3} \int_{\Omega}\left|\beta w_{0}+v\right|^{p} d x+\frac{c_{1}}{3}|\Omega|+\frac{2}{p}\left|\beta w_{0}\right|^{p}|\Omega|\left(\frac{6 c_{2}}{c_{1} q}\right)^{\frac{p}{q}}+ \\
& +\frac{c_{1}}{3} \int_{\Omega}\left|\beta w_{0}+v\right|^{p} d x+\frac{2}{q}\left(\frac{6 \alpha}{c_{1} p}\right)^{\frac{\alpha q}{p-\alpha q}}|\Omega| \frac{p-\alpha q}{p}+\frac{2}{p}\left|\beta w_{0}\right|^{p}|\Omega| .
\end{aligned}
$$

Logo, de (3.78), podemos escrever

$$
\begin{aligned}
& \frac{d}{d t}\|v\|_{L^{2}(\Omega)}^{2}+2 d \lambda_{1}\|v\|_{L^{2}(\Omega)}^{2} \leqslant 2 c_{3}|\Omega|+2|\Omega|\left(\frac{6(\alpha+1)}{c_{1} p}\right)^{\frac{\alpha+1}{p-(\alpha+1)}} \frac{p-(\alpha+1)}{p}+ \\
& +\frac{c_{1}}{3}|\Omega|+\frac{2}{p}\left(\frac{6 c_{2}}{c_{1} q}\right)^{\frac{p}{q}} K^{p}+\frac{2}{q}\left(\frac{6 \alpha}{c_{1} p}\right)^{\frac{\alpha q}{p-\alpha q}}|\Omega| \frac{p-\alpha q}{p}+\frac{2}{p} K^{p} .
\end{aligned}
$$

De (3.79), decorre que

$$
\|T(t) v\|_{L^{\infty}\left(0, T ; L^{2}(\Omega)\right)}^{2} \leqslant e^{-2 d \lambda_{1} t}\|v(0)\|_{L^{2}(\Omega)}^{2}+\frac{k_{1}}{2 d \lambda_{1}}
$$

para $t \geqslant 0$ e para todo $T>0$, onde

$$
\begin{aligned}
& k_{1}=2 c_{3}|\Omega|+2|\Omega|\left(\frac{6(\alpha+1)}{c_{1} p}\right)^{\frac{\alpha+1}{p-(\alpha+1)}} \frac{p-(\alpha+1)}{p}+\frac{c_{1}}{3}|\Omega|+ \\
& +\frac{2}{p}\left(\frac{6 c_{2}}{c_{1} q}\right)^{\frac{p}{q}} K^{p}+\frac{2}{q}\left(\frac{6 \alpha}{c_{1} p}\right)^{\frac{\alpha q}{p-\alpha q}}|\Omega| \frac{p-\alpha q}{p}+\frac{2}{p} K^{p} .
\end{aligned}
$$

Se $d \rightarrow+\infty$, então de (3.80), temos

$$
\|T(t) v\|_{L^{\infty}\left(0, T ; L^{2}(\Omega)\right)}^{2} \rightarrow 0 .
$$

Em particular, se $d \rightarrow \infty$, então $v \stackrel{*}{\rightarrow} 0$ em $L^{\infty}\left(0, T ; L^{2}(\Omega)\right)$, para todo $T>0$.

Se tomarmos o produto da primeira equação do sistema (3.76) por $-\Delta v$ e integrarmos em $\Omega$, como fizemos na página 70 , resulta que

$$
-\int_{\Omega} v_{t} \Delta v d x=-d \int_{\Omega} \Delta v \Delta v d x+\int_{\Omega} f\left(\beta w_{0}+v\right) \Delta v d x-\int_{\Omega}\left|\beta w_{0}+v\right|^{\alpha-1}\left(\beta w_{0}+v\right) \Delta v d x-
$$




$$
-\frac{1}{|\Omega|} \int_{\Omega}\left[f\left(\beta w_{0}+v\right)-\left|\beta w_{0}+v\right|^{\alpha-1}\left(\beta w_{0}+v\right)\right] d x \int_{\Omega} \Delta v d x
$$

Observemos que $\int_{\Omega} \Delta v d x=\int_{\Gamma} \frac{\partial v}{\partial \nu} d \Gamma=0$. Além disso, usando integração por partes e a hipótese (3.69), obtemos

$$
-\int_{\Omega} f\left(\beta w_{0}+v\right) \Delta v \geqslant c_{f} \int_{\Omega}|\nabla v|^{2} d x
$$

e

$$
\int_{\Omega}\left|\beta w_{0}+v\right|^{\alpha}|\Delta v| d x \stackrel{\text { Young }}{\leqslant} \frac{\alpha}{2 d}\left\|\beta w_{0}+v\right\|_{L^{2}(\Omega)}^{2}+\frac{(1-\alpha)}{2 d}|\Omega|+\frac{d}{2} \int_{\Omega}|\Delta v|^{2} d x
$$

Substituindo (3.83) e (3.84) em (3.82), obtemos

$$
\frac{d}{d t}\|\nabla v\|_{L^{2}(\Omega)}^{2}+d\|\Delta v\|_{L^{2}(\Omega)}^{2}+2 c_{f} \int_{\Omega}|\nabla v|^{2} d x \leqslant \frac{\alpha}{d}\left(\left\|\beta w_{0}+v\right\|_{L^{\infty}\left(0, T ; L^{2}(\Omega)\right)}\right)^{2}+\frac{(1-\alpha)}{d}|\Omega|,
$$

para todo $T>0$.

Aplicando a desigualdade $\|\Delta v\|_{L^{2}(\Omega)}^{2} \geqslant \lambda_{1}\|\nabla v\|_{L^{2}(\Omega)}^{2}$ em (3.85), obtemos

$$
\frac{d}{d t}\|\nabla v\|_{L^{2}(\Omega)}^{2}+\lambda_{1} d\|\nabla u\|_{L^{2}(\Omega)}^{2} \leqslant \frac{\alpha}{d} K^{2}+\frac{(1-\alpha)}{d}|\Omega|
$$

De (3.86), temos

$$
\|T(t) \nabla v\|_{L^{\infty}\left(0, T ; L^{2}(\Omega)\right)}^{2} \leqslant\|\nabla v(0)\|_{L^{2}(\Omega)}^{2} e^{-\lambda_{1} d t}+\frac{\alpha}{\lambda_{1} d^{2}} K^{2}+\frac{(1-\alpha)|\Omega|}{\lambda_{1} d^{2}}
$$

para $t \geqslant 0$ e para todo $T>0$, donde

$$
\|T(t) \nabla v\|_{L^{\infty}\left(0, T ; L^{2}(\Omega)\right)}^{2} \rightarrow 0
$$

quando $d \rightarrow+\infty$, para todo $T>0$. Em particular, se $d \rightarrow+\infty$, então $\nabla v \stackrel{*}{\rightarrow} 0$ em $L^{\infty}\left(0, T ; L^{2}(\Omega)\right)$, para todo $T>0$.

De $(3.81)$ e $(3.88)$, decorre que $\|T(t) v\|_{L^{\infty}\left(\mathbb{R}_{+} ; H^{1}(\Omega)\right)} \rightarrow 0$ quando $d \rightarrow+\infty$ e, em particular, $v \stackrel{*}{\rightarrow} 0$ em $L^{\infty}\left(0, T ; H^{1}(\Omega)\right)$ quando $d \rightarrow+\infty$, para todo $T>0$. Podemos afirmar também que $\|v\|_{L^{2}\left(0, T ; H^{1}(\Omega)\right)} \rightarrow 0$ e $v \rightarrow 0$ em $L^{2}\left(0, T ; H^{1}(\Omega)\right)$ quando $d \rightarrow \infty$, para todo $T>0$.

Agora, pela desigualdade de Gagliardo-Nirenberg 1.1.4 existe uma constante positiva 
$k_{2}$ tal que

$$
\int_{0}^{T}\|v(t)\|_{L^{p}(\Omega)}^{p} d t \leqslant k_{2}^{p} \int_{0}^{T}\|v(t)\|_{L^{2}(\Omega)}^{(1-\theta) p}\|v(t)\|_{H_{d}^{1}(\Omega)}^{\theta p} d t \leqslant k_{2}^{p}\|v\|_{L^{\infty}\left(0, T ; L^{2}(\Omega)\right)}^{(1-\theta) p}\|v\|_{L^{\infty}\left(0, T ; H^{1}(\Omega)\right)}^{\theta p} T
$$

donde obtemos que

$$
\|v\|_{L^{p}\left(0, T ; L^{p}(\Omega)\right)} \rightarrow 0
$$

quando $d \rightarrow+\infty$, para todo $T>0$, o que implica que $v \rightarrow 0$ em $L^{p}\left(0, T ; L^{p}(\Omega)\right)$ quando $d \rightarrow+\infty$, para todo $T>0$.

Pela continuidade do operador derivação $\partial_{t}$ de $\mathcal{D}^{\prime}\left(0, T ; L^{p}(\Omega)\right) \cap \mathcal{D}^{\prime}\left(0, T ; H^{1}(\Omega)\right)$ em $\mathcal{D}^{\prime}\left(0, T ; L^{p}(\Omega)\right) \cap \mathcal{D}^{\prime}\left(0, T ; H^{1}(\Omega)\right)$, obtemos

$$
\partial_{t} v \rightarrow 0 \quad \text { em } \mathcal{D}^{\prime}\left(0, T ; L^{p}(\Omega)\right) \cap \mathcal{D}^{\prime}\left(0, T ; H^{1}(\Omega)\right)
$$

quando $d \rightarrow+\infty$, para todo $T>0$.

Como $\partial_{t} v \in L^{q}\left(0, T ; H^{-r}(\Omega)\right)$, resulta que

$$
\partial_{t} v \rightarrow 0 \quad \text { em } L^{q}\left(0, T ; H^{-r}(\Omega)\right)
$$

quando $d \rightarrow \infty$, para todo $T>0$.

Concluímos que $v \rightarrow 0$ na topologia $\Theta_{l o c}^{+}$quando $d \rightarrow+\infty$.

Vamos demonstrar que $|G(\beta, v)-G(\beta, 0)| \rightarrow 0$ quando $v \rightarrow 0$ em $\Theta_{l o c}^{+}$, ou seja, $G$ é uma aplicação contínua com respeito a $v$. De fato, do Lema 3.3.1 e da hipótese (3.70) temos que

$$
\begin{aligned}
& |G(\beta, v)-G(\beta, 0)| \leqslant \frac{1}{\sqrt{\Omega}} \int_{\Omega}\left|f\left(\beta w_{0}+v\right)-f\left(\beta w_{0}\right)\right| d x+ \\
& +\frac{1}{\sqrt{|\Omega|}} \int_{\Omega} \| \beta w_{0}+\left.v\right|^{\alpha-1}\left(\beta w_{0}+v\right)-\left|\beta w_{0}\right|^{\alpha-1} \beta w_{0}\left|d x \leqslant \frac{C}{\sqrt{|\Omega|}} \int_{\Omega}\right| v \mid d x+ \\
& +\frac{C \max \left\{1,2^{p-3}\right\}}{\sqrt{|\Omega|}} \int_{\Omega}|v|\left(\left|\beta w_{0}\right|^{p-2}+|v|^{p-2}\right) d x+\frac{C}{\sqrt{|\Omega|}} \int_{\Omega}\left|v \| \beta w_{0}\right|^{p-2} d x+\frac{2^{1-\alpha}}{\sqrt{|\Omega|}} \int_{\Omega}|v|^{\alpha} d x \\
& \leqslant C\|v\|_{L^{\infty}\left(0, T ; L^{2}(\Omega)\right)}+C \max \left\{1,2^{p-3}\right\}\left(\left|\beta w_{0}\right|^{2}+1\right)^{p-2}\|v\|_{L^{\infty}\left(0, T ; L^{2}(\Omega)\right)}+ \\
& +C|\Omega|^{-\frac{1}{2}} \max \left\{1,2^{p-3}\right\}\left(k_{1} k_{2}\right)^{p-1}\left(\|v\|_{L^{\infty}\left(0, T ; H^{1}(\Omega)\right)}\right)^{p-1}+ \\
& +C(K+1)^{p-2}\|v\|_{L^{\infty}\left(0, T ; L^{2}(\Omega)\right)}+2^{1-\alpha}|\Omega|^{\frac{1-\alpha}{2}}\left(\|v\|_{L^{\infty}\left(0, T ; L^{2}(\Omega)\right)}\right)^{\alpha} \\
& \leqslant C\|v\|_{L^{\infty}\left(0, T ; L^{2}(\Omega)\right)}+C \max \left\{1,2^{p-3}\right\}\left(K^{2}+1\right)^{p-2}\|v\|_{L^{\infty}\left(0, T ; L^{2}(\Omega)\right)}+ \\
& +C|\Omega|^{-\frac{1}{2}} \max \left\{1,2^{p-3}\right\}\left(k_{1} k_{2}\right)^{p-1}\left(\|v\|_{L^{\infty}\left(0, T ; H^{1}(\Omega)\right)}\right)^{p-1}+ \\
& +C\left(\left|\beta w_{0}\right|^{2}+1\right)^{p-2}\|v\|_{L^{\infty}\left(0, T ; L^{2}(\Omega)\right)}+2^{1-\alpha}|\Omega|^{\frac{1-\alpha}{2}}\left(\|v\|_{L^{\infty}\left(0, T ; L^{2}(\Omega)\right)}\right)^{\alpha}
\end{aligned}
$$


para todo $T>0$, onde $k_{1}$ e $k_{2}$ são constantes de imersão. Como $\|v\|_{L^{\infty}\left(0, T ; L^{2}(\Omega)\right)} \rightarrow 0 \mathrm{e}$ $\|v\|_{L^{\infty}\left(0, T ; H_{d}^{1}(\Omega)\right)} \rightarrow 0$ quando $d \rightarrow \infty$, para todo $T>0$, resulta de $(3.90)$ que $\mid G(\beta, v)-$ $G(\beta, 0) \mid \rightarrow 0$ quando $v \rightarrow 0$ em $\Theta_{l o c}^{+}$.

Observemos que a equação diferencial ordinária $\xi_{t}=-f(\xi)+|\xi|^{\alpha-1} \xi$ possui um atrator de trajetórias $\mathfrak{A}_{\infty}$. Pelo Teorema 2.1.12 isso é verdade desde que a condição de dissipatividade (2.2) seja satisfeita. De fato, pela desigualdade de Young (1.3) podemos obter constantes positivas $k_{1}$ e $k_{2}$ tais que

$$
\left(f(\xi)-|\xi|^{\alpha-1} \xi\right) \cdot \xi \geqslant k_{1}|\xi|^{2}-k_{2} .
$$

Temos que

$$
k_{1}=\frac{1}{2} \quad \text { e } \quad k_{2}=\left(\frac{2}{c_{1} p}\right)^{\frac{2}{p-2}} \frac{p-2}{p}+(\alpha+1)^{\frac{\alpha+1}{1-\alpha}} \frac{1-\alpha}{2} .
$$

Observemos finalmente que o atrator $\mathfrak{A}_{\infty}$ pode ser imerso no espaço $\left[w_{0}\right] \oplus Y$ por meio da aplicação satisfazendo $\xi \rightarrow \xi w_{0}$. O atrator imerso será denotado por $\widetilde{\mathfrak{A}_{\infty}}$.

Do que fizemos antes, para toda vizinhança $\mathcal{O}$ do atrator $\widetilde{\mathfrak{A}_{\infty}}$ na topologia $\Theta_{l o c}^{+}$existe $d_{0}>0$ tal que $\mathfrak{A}_{d} \subset \mathcal{O}\left(\widetilde{\mathfrak{A}_{\infty}}\right)$ para $d \geqslant d_{0}$. 


\section{Atrator de trajetórias de uma equação hiperbólica}

O fenômeno de homogeneização espacial das soluções de equações parabólicas sujeitas a condição de Neumann relatado no início do Capítulo 3 foi estudado também para problemas hiperbólicos. Algumas referências sobre esse assunto são Sola-Morales,J.; Valencia, M. [44], Pereira, A. L.; de Oliveira, L. A. F. [39] e Carvalho, A. N. [7].

Por exemplo, em Sola-Morales,J.; Valencia, M. [44] considerou-se a seguinte equação de onda semilinear com amortecimento

$$
\left\{\begin{array}{l}
u_{t t}+2 \gamma u_{t}=d \Delta u+f(u, t) \quad(t, x) \in\left[t_{0},+\infty\right) \times \Omega \\
\frac{\partial u}{\partial \nu}=0 \quad x \in \Gamma=\partial \Omega
\end{array}\right.
$$

onde $u(t, x) \in \mathbb{R}, \Omega \subset \mathbb{R}^{n}$ é um subconjunto aberto, limitado, conexo e com fronteira suave $\Gamma, \gamma>0, d>0$ e $f$ é uma função suave, globalmente Lipschitz com respeito a $u$ e com constante de Lipschitz $L$. O resultado principal obtido pelos autores é transcrito a seguir

Teorema 4.0.3. Seja $\sigma=d \lambda-L$, onde $\lambda$ é o menor autovalor positivo do operador $-\Delta$ em $\Omega$ com condição de fronteira Neumann homogênea e suponhamos que $\sigma>\frac{L^{2}}{4 \gamma^{2}}$. Se $u:\left[t_{0},+\infty\right) \times \Omega \rightarrow \mathbb{R}$ é uma solução da equação (4.1), então

$(i)\|u(t, \cdot)-\bar{u}(t)\|_{H^{1}(\Omega)} \rightarrow 0$ quando $t \rightarrow+\infty$, onde $\bar{u}(t)$ é a média espacial de $u(t, x)$;

(ii) $\bar{u}(t)$ satisfaz $\bar{u}^{\prime \prime}+2 \gamma \bar{u}^{\prime}(t)=f(t, \bar{u})+g(t) \operatorname{com} g(t) \rightarrow 0$ quando $t \rightarrow+\infty$.

É importante observar que os trabalhos citados acima lidam com problemas que gozam da propriedade de unicidade de solução. Não encontramos referências na literatura no contexto de problemas de Cauchy sem unicidade e envolvendo o atrator de trajetórias. Assim, nosso principal objetivo neste capítulo é dar nossa contribuição ao estudo do comportamento assintótico das soluções de problemas hiperbólicos com difusão grande e com condição de Neumann homogênea, cujo problema de Cauchy correspondente não satisfaz algum resultado 
4 Atrator de trajetórias de uma equação hiperbólica

de unicidade. A nossa pesquisa vai se restringir ao seguinte problema

$$
\left\{\begin{array}{l}
\frac{\partial^{2} u}{\partial t^{2}}+2 \gamma \frac{\partial u}{\partial t}=d \Delta u-f(u)+|u|^{\alpha-1} u \\
\frac{\partial u}{\partial \nu}=0 \quad(t, x) \in(0, \infty) \times \Gamma,
\end{array}\right.
$$

onde $\Omega \subset \mathbb{R}^{n}(n \geqslant 3)$ é um subconjunto aberto, limitado, conexo, com fronteira suave $\Gamma \mathrm{e}$ para cada ponto $x \in \Gamma, \nu=\nu(x)$ indica o vetor normal exterior unitário a $\Gamma$ no ponto $x$. Além disso, $\gamma>0, d>0, \alpha \in(0,1)$ e $f: \mathbb{R} \rightarrow \mathbb{R}$ é uma função de classe $C^{1}$ satisfazendo as seguintes hipóteses: existem constantes positivas $C, \gamma_{0}, \gamma_{1}, \gamma_{2}, c_{1}, c_{2}$ e $2<p<\frac{2 n}{n-2}$ tais que

$$
\begin{gathered}
\left|f\left(u_{1}\right)-f\left(u_{2}\right)\right| \leqslant C\left|u_{1}-u_{2}\right|\left(1+\left|u_{1}\right|^{p-2}+\left|u_{2}\right|^{p-2}\right), \\
|f(u)| \leqslant \gamma_{0}\left(|u|^{p-1}+1\right), \\
F(u)=\int_{0}^{u} f(w) d w, \quad F(u) \geqslant \gamma_{1}|u|^{p}-c_{1}, \\
f(u) \cdot u \geqslant \gamma_{2} F(u)-c_{2},
\end{gathered}
$$

para quaisquer $u, u_{1}, u_{2} \in \mathbb{R}$.

Vamos começar aplicando o roteiro da Seção 2.2 na construção do atrator de trajetórias $\mathfrak{A}_{d}$. Isso será feito na Seção 4.1 sem a hipótese (4.3) que é desnecessária nesse ponto.

Na Seção 4.2 vamos considerar a equação (4.2) com um parâmetro pequeno $\epsilon$ e construiremos o correspondente atrator de trajetórias. A princípio faremos isso para o problema a seguir, onde o parâmetro aparece na parte não Lipschitz do termo de reação

$$
\left\{\begin{array}{l}
\frac{\partial^{2} u}{\partial t^{2}}+2 \gamma \frac{\partial u}{\partial t}=d \Delta u-f(u)+\epsilon|u|^{\alpha-1} u \quad(t, x) \in(0, \infty) \times \Omega \\
\frac{\partial u}{\partial \nu}=0 \quad(t, x) \in(0, \infty) \times \Gamma
\end{array}\right.
$$

e depois no caso em que o parâmetro aparece multiplicando o termo da derivada de maior ordem

$$
\left\{\begin{array}{l}
\epsilon \frac{\partial^{2} u}{\partial t^{2}}+2 \gamma u_{t}=d \Delta u-f(u)+|u|^{\alpha-1} u \quad(t, x) \in(0, \infty) \times \Omega \\
\frac{\partial u}{\partial \nu}=0 \quad(t, x) \in(0, \infty) \times \Gamma
\end{array}\right.
$$

e verificaremos que o atrator de trajetórias do problema envolvendo o parâmetro $\epsilon$ com as hipóteses (4.4)-(4.6) está contido numa vizinhança do atrator de trajetórias do respectivo problema limite quando $\epsilon \rightarrow 0^{+}$com respeito a topologia que denotaremos por $\Theta_{l o c}^{+}$. 
E, finalmente, na Seção 4.3 vamos adotar o sistema ortonormal completo para $L^{2}(\Omega)$ dado pelas autofunções $\left\{w_{j}\right\}_{j=0}^{\infty}$ do problema de Neumann

$$
\left\{\begin{array}{l}
\Delta w+\lambda w=0 \quad \text { em } \Omega \\
\left.\frac{\partial w}{\partial \nu}\right|_{\Gamma}=0
\end{array}\right.
$$

e indicaremos por $Y$ o espaço das funções ortogonais a $w_{0}$. Por meio da decomposição do espaço $L^{2}(\Omega)=\left[w_{0}\right] \oplus Y$ vamos reescrever a equação (4.2) como um sistema de equações. Considerando a equação diferencial ordinária

$$
\left\{\begin{array}{l}
\xi^{\prime \prime}(t)+2 \gamma \xi^{\prime}(t)=-f(\xi(t))+|\xi(t)|^{\alpha-1} \xi(t) \\
\xi(0)=\xi_{0} \in \mathbb{R}
\end{array}\right.
$$

e o seu atrator de trajetórias $\mathfrak{A}_{\infty}$, demonstraremos que a família $\left\{\mathfrak{A}_{d}\right\}_{d} \cup \widetilde{\mathfrak{A}_{\infty}}$ é semicontínua superiormente em $d=+\infty$, onde $\widetilde{\mathfrak{A}_{\infty}}$ é a imersão de $\mathfrak{A}_{\infty}$ em $\left[w_{0}\right] \oplus Y$.

\subsection{Construção do atrator de trajetórias de um pro- blema hiperbólico}

Seja $\Omega$ um subconjunto aberto, limitado, conexo do $\mathbb{R}^{n}(n \geqslant 3)$ e com fronteira suave $\Gamma$. Para cada ponto $x \in \Gamma$, indicaremos por $\nu=\nu(x)$ o vetor normal exterior unitário a $\Gamma$ no ponto $x$. Consideremos o seguinte problema hiperbólico com condição de Neumann

$$
\left\{\begin{array}{l}
\frac{\partial^{2} u}{\partial t^{2}}+2 \gamma \frac{\partial u}{\partial t}=d \Delta u-f(u)+|u|^{\alpha-1} u \quad(t, x) \in(0, \infty) \times \Omega \\
\frac{\partial u}{\partial \nu}=0 \quad(t, x) \in(0, \infty) \times \Gamma
\end{array}\right.
$$

onde $\gamma>0, d>0$ está fixado, $\alpha \in(0,1)$ e $f: \mathbb{R} \rightarrow \mathbb{R}$ é uma função de classe $C^{1}$ satisfazendo satisfazendo as seguintes hipóteses: existem constantes positivas $\gamma_{0}, \gamma_{1}, \gamma_{2}, c_{1}, c_{2}$ e $2<p<$ $\frac{2 n}{n-2}$ tais que

$$
\begin{gathered}
|f(u)| \leqslant \gamma_{0}\left(|u|^{p-1}+1\right), \\
F(u)=\int_{0}^{u} f(w) d w, \quad F(u) \geqslant \gamma_{1}|u|^{p}-c_{1}, \\
f(u) \cdot u \geqslant \gamma_{2} F(u)-c_{2},
\end{gathered}
$$

para qualquer $u \in \mathbb{R}$. 
Como fizemos na Seção 3.1, vamos especificar os espaços em que trabalharemos e definiremos quando uma função $u=u(t, x)$ será solução de (4.10). Como não há um resultado de unicidade de solução para o problema (4.10) empregaremos a teoria do atrator de trajetórias em nosso estudo, adaptando resultados obtidos nas referências Chepyzhov, V. V. and Vishik, M. I. [9], [17], onde problemas similares a (4.10) foram considerados para a condição de Dirichlet.

Consideremos $\tilde{f}(u)=f(u)-|u|^{\alpha-1} u$. Das hipóteses (4.11)-(4.13) podemos afirmar que existem constantes positivas $\tilde{\gamma_{0}}, \tilde{\gamma_{1}}, \tilde{\gamma_{2}}, \tilde{c_{1}}, \tilde{c_{2}}$ tais que

$$
\begin{gathered}
|\tilde{f}(u)| \leqslant\left(\gamma_{0}+1\right)\left(|u|^{p-1}+1\right) \doteq \tilde{\gamma_{0}}\left(|u|^{p-1}+1\right) \\
\tilde{F}(u)=\int_{0}^{u} \tilde{f}(w) d w, \quad \tilde{F}(u) \geqslant \tilde{\gamma_{1}}|u|^{p}-\tilde{c_{1}},
\end{gathered}
$$

onde

$$
\begin{gathered}
\tilde{\gamma_{1}}=\frac{\gamma_{1}}{2} \quad \text { e } \tilde{c_{1}}=c_{1}+\frac{1}{\alpha+1}\left(\frac{2}{\gamma_{1} p}\right)^{\frac{\alpha+1}{p-(\alpha+1)}}\left(\frac{p-(\alpha+1)}{p}\right) ; \\
\tilde{f}(u) \cdot u \geqslant \tilde{\gamma_{2}}|u|^{p}-\tilde{c_{2}},
\end{gathered}
$$

onde

$$
\tilde{\gamma_{2}}=\frac{\gamma_{1} \gamma_{2}}{2} \quad \text { e } \quad \tilde{c_{2}}=\left[\gamma_{2} c_{1}+c_{2}+\left(\frac{2(\alpha+1)}{\gamma_{1} \gamma_{2} p}\right)^{\frac{\alpha+1}{p-(\alpha+1)}}\left(\frac{p-(\alpha+1)}{p}\right)\right]
$$

Vamos fazer algumas observações antes de introduzir a definição de solução para o nosso problema.

Assim como fizemos no Capítulo 3 vamos denotar por $H_{d}^{1}(\Omega)$ o $H^{1}(\Omega)$ munido da norma (3.14).

Observemos que para $c_{3}=\tilde{c_{1}}+1$ existe uma constante positiva $K$ tal que a função $\Phi(u)=\left(\tilde{F}(u)+c_{3}\right)^{\frac{1}{p}}$ é Lipschitz. De fato, de (4.14), (4.15) e do Teorema do Valor Médio, obtemos

$$
\left|\Phi\left(u_{1}\right)-\Phi\left(u_{2}\right)\right| \leqslant\left|\Phi^{\prime}(u)\right|\left|u_{1}-u_{2}\right|
$$

para quaisquer $u_{1}, u_{2} \in \mathbb{R}$ e $\left|\Phi^{\prime}(u)\right| \leqslant \frac{\tilde{\gamma_{0}}}{p}\left(\frac{1}{\tilde{\gamma_{1}}}\right)^{\frac{p-1}{p}}$ quando $|u| \rightarrow+\infty$. Se existir $u_{0}>0$ tal que $|u| \leqslant u_{0}$, então existe constante $k>0$ tal que $\left|\Phi^{\prime}(u)\right| \leqslant k$. Logo, se tomarmos 
$K=\max \left\{k, \frac{\tilde{\gamma_{0}}}{p}\left(\frac{1}{\tilde{\gamma}_{1}}\right)^{\frac{p-1}{p}}\right\}$, resulta que

$$
\left|\Phi\left(u_{1}\right)-\Phi\left(u_{2}\right)\right| \leqslant K\left|u_{1}-u_{2}\right|
$$

Seja $q$ o expoente conjugado de $p$, isto é, $\frac{1}{p}+\frac{1}{q}=1$. Observemos que se $u \in L^{\infty}\left(0, T ; L^{p}(\Omega)\right)$, então $|u|^{\alpha-1} u \in L^{\infty}\left(0, T ; L^{q}(\Omega)\right)$ e de (4.14) decorre que $\tilde{f}(u) \in L^{\infty}\left(0, T ; L^{q}(\Omega)\right)$. Além disso, existe uma constante $\tilde{\tilde{\gamma}}_{0}$ tal que

$$
\|\tilde{f}(u)\|_{L^{\infty}\left(0, T ; L^{q}(\Omega)\right)}^{q} \leqslant \tilde{\tilde{\gamma}}_{0}\left(\|u\|_{L^{\infty}\left(0, T ; L^{p}(\Omega)\right)}^{p}+1\right)
$$

para todo $T>0$, onde $\tilde{\tilde{\gamma}_{0}}=2^{q} \tilde{\gamma}_{0}^{q}(|\Omega|+1)$.

Por outro lado, se $u \in L^{\infty}\left(0, T ; H_{d}^{1}(\Omega)\right)$, então $\Delta u \in L^{\infty}\left(0, T ; H_{d}^{-1}(\Omega)\right)$. Como $p>2$, do Teorema de imersão de Sobolev e por dualidade temos $L^{q}(\Omega) \subset H^{-r}(\Omega)$, onde $r=\max \left\{1, n\left(\frac{1}{q}-\frac{1}{2}\right)\right\}$. Logo, se $u \in L^{\infty}\left(0, T ; L^{p}(\Omega)\right) \cap L^{\infty}\left(0, T ; H_{d}^{1}(\Omega)\right)$, temos que a equação (4.10) pode ser considerada no sentido de distribuição do espaço $D^{\prime}\left(\mathbb{R}_{+} ; H^{-r}(\Omega)\right)$.

Definição 4.1.1. Uma função $u=u(t, x), t \in[0, T], x \in \Omega$, é uma solução fraca global de $(4.10)$ se $u \in L^{\infty}\left(0, T ; L^{p}(\Omega)\right) \cap L^{\infty}\left(0, T ; H_{d}^{1}(\Omega)\right), u_{t} \in L^{\infty}\left(0, T ; L^{2}(\Omega)\right)$ e u satisfaz a equação (4.10) no sentido de distribuição do espaço $D^{\prime}\left(0, T ; H^{-r}(\Omega)\right)$, para todo $T>0$, onde $r=\max \left\{1, n\left(\frac{1}{q}-\frac{1}{2}\right)\right\}$, isto é, para toda $\varphi \in H_{d}^{1}(\Omega) \cap L^{p}(\Omega)$ vale a seguinte igualdade

$$
\begin{aligned}
& \frac{d^{2}}{d t^{2}} \int_{\Omega} u(t, x) \cdot \varphi(x) d x+2 \gamma \frac{d}{d t} \int_{\Omega} u(t, x) \cdot \varphi d x+\int_{\Omega}[d \nabla u(t, x) \cdot \nabla \varphi(x)+f(u(t, x)) \cdot \varphi(x)- \\
&\left.-|u(t, x)|^{\alpha-1} u(t, x) \cdot \varphi(x)\right] d x=0 .
\end{aligned}
$$

Observação 4.1.1. Sabemos que se u é uma solução fraca global da equação (4.10), então $u \in L^{\infty}\left(0, T ; L^{p}(\Omega)\right) \cap L^{\infty}\left(0, T ; H_{d}^{1}(\Omega)\right) \hookrightarrow L^{\infty}\left(0, T ; L^{2}(\Omega)\right)$ e $u_{t} \in L^{\infty}\left(0, T ; L^{2}(\Omega)\right)$ para todo $T>0$, portanto do Lema 1.1 .13 resulta que $u \in C\left(0, T ; L^{2}(\Omega)\right)$. Temos também que $u_{t} \in L^{\infty}\left(0, T ; L^{2}(\Omega)\right) \hookrightarrow L^{\infty}\left(0, T ; H^{-r}(\Omega)\right)$ e $u_{t t} \in L^{\infty}\left(0, T ; H^{-r}(\Omega)\right)$ para todo $T>0$, donde concluímos novamente pelo Lema 1.1 .13 que $u_{t} \in C\left(0, T ; H^{-r}(\Omega)\right)$.

Proposição 4.1.2. Se u é uma solução fraca global de (4.10), então

$$
u \in C_{w}\left(0, T ; H_{d}^{1}(\Omega)\right), \quad u \in C_{w}\left(0, T ; L^{p}(\Omega)\right), \quad u_{t} \in C_{w}\left(0, T ; L^{2}(\Omega),\right.
$$

para todo $T>0$. Além disso, para todo $\delta \in \mathbb{R}$ a função

$$
t \mapsto\|u(t)\|_{H_{d}^{1}(\Omega)}+\left\|u_{t}(t)+\delta u(t)\right\|_{L^{2}(\Omega)}+\|u(t)\|_{L^{p}(\Omega)}
$$

é semicontínua inferiormente para $t \in[0, T]$, para todo $T>0$.

Demonstração: Como $H_{d}^{1}(\Omega) \cap L^{p}(\Omega) \hookrightarrow L^{2}(\Omega)$ e $u \in C_{w}\left(0, T ; L^{2}(\Omega)\right)$, segue diretamente 
do Teorema 1.1.18, item $(i)$, que $u \in C_{w}\left(0, T ; H_{d}^{1}(\Omega)\right)$ e $u \in C_{w}\left(0, T ; L^{p}(\Omega)\right)$. Além disso, $L^{2}(\Omega) \hookrightarrow H^{-r}(\Omega)$ e $u_{t} \in C_{w}\left(0, T ; H^{-r}(\Omega)\right)$, donde $u_{t} \in C_{w}\left(0, T ; L^{2}(\Omega)\right)$.

A expressão $\left(\|u(t)\|_{H_{d}^{1}(\Omega)}+\left\|u_{t}(t)+\delta u(t)\right\|_{L^{2}(\Omega)}\right)^{\frac{1}{2}}$ define uma norma equivalente em $H_{d}^{1}(\Omega) \times L^{2}(\Omega)$. Logo, do Teorema 1.1.18, item (ii), resulta que $\left(\|u(t)\|_{H_{d}^{1}(\Omega)}+\left\|u_{t}(t)+\delta u(t)\right\|_{L^{2}(\Omega)}\right)^{\frac{1}{2}}$ é uma função semicontínua inferiormente em $[0, T]$. O mesmo é valido para $\|u(t)\|_{L^{p}(\Omega)}$ e $\|u(t)\|_{L^{p}(\Omega)}^{p}$. Como a soma de funções semicontínuas é ainda uma função semicontínua, segue a semicontinuidade inferior de (4.20).

Sejam $u \in H_{d}^{1}(\Omega) \cap L^{p}(\Omega)$ e $v \in L^{2}(\Omega)$. Definamos $I:\left(H_{d}^{1}(\Omega) \cap L^{p}(\Omega)\right) \times L^{2}(\Omega) \rightarrow \mathbb{R}$ por

$$
I(u, v)=\frac{1}{2} \int_{\Omega}\left[|v|^{2}+d|\nabla u|^{2}+2 \tilde{F}(u)\right] d x
$$

Proposição 4.1.3. Existem constantes positivas $k_{1}, k_{2}, k_{3}, k_{4}$ tais que

$$
k_{1}\left[\|u\|_{H_{d}^{1}(\Omega)}^{2}+\|v\|_{L^{2}(\Omega)}^{2}+\|u\|_{L^{p}(\Omega)}^{p}\right]-k_{2} \leqslant I(u, v)
$$

e

$$
I(u, v) \leqslant k_{3}\left[\|u\|_{H_{d}^{1}(\Omega)}^{2}+\|v\|_{L^{2}(\Omega)}^{2}+\|u\|_{L^{p}(\Omega)}^{p}\right]+k_{4} .
$$

Demonstração: De fato, usando a hipótese (4.15) e a desigualdade de Young (1.3) obtemos

$$
\begin{aligned}
I(u, v) & \geqslant \frac{1}{2} \min \left\{1, \tilde{\gamma_{1}}\right\}\left[\|u\|_{H_{d}^{1}(\Omega)}^{2}+\|v\|_{L^{2}(\Omega)}^{2}+\|u\|_{L^{p}(\Omega)}^{p}\right]- \\
& -\left[\tilde{c_{1}}|\Omega|+\frac{1}{2}\left(\frac{2}{\tilde{\gamma_{1} p}}\right)^{\frac{2}{p-2}}|\Omega|\left(\frac{p-2}{p}\right)\right] .
\end{aligned}
$$

Se $\tilde{L}$ é uma constante positiva tal que $|\tilde{F}(u)| \leqslant \tilde{L}\left(1+|u|^{p}\right)$, então

$$
I(u, v) \leqslant \frac{1}{2} \max \{1,2 \tilde{L}\}\left[\|u\|_{H_{d}^{1}(\Omega)}^{2}+\|v\|_{L^{2}(\Omega)}^{2}+\|u\|_{L^{p}(\Omega)}^{p}\right]+\tilde{L}|\Omega| .
$$

Portanto,

$$
\begin{gathered}
k_{1}=\frac{1}{2} \min \left\{1, \tilde{\gamma_{1}}\right\}, \quad k_{2}=\tilde{c_{1}}|\Omega|+\frac{1}{2}\left(\frac{2}{\tilde{\gamma_{1}} p}\right)^{\frac{2}{p-2}}|\Omega|\left(\frac{p-2}{p}\right) \\
k_{3}=\frac{1}{2} \max \{1,2 \tilde{L}\}, \quad k_{4}=\tilde{L}|\Omega| .
\end{gathered}
$$


Seja

$$
0<\delta<\min \left\{2 \gamma, 2 k_{1}\right\}
$$

onde $k_{1}=\frac{1}{2} \min \left\{1, \tilde{\gamma}_{1}\right\}$. Consideremos o seguinte funcional

$$
J_{\delta}(u, v)=\frac{1}{2} \int_{\Omega}\left[|v|^{2}+d|\nabla u|^{2}+2 \tilde{F}(u)+2 \delta u \cdot v+2 \delta \gamma|u|^{2}\right] d x .
$$

Proposição 4.1.4. O funcional $J_{\delta}$ satisfaz

$$
\left(k_{1}-\frac{\delta}{2}\right)\left[\|u\|_{H_{d}^{1}(\Omega)}^{2}+\|v\|_{L^{2}(\Omega)}^{2}+\|u\|_{L^{p}(\Omega)}^{p}\right]-k_{2} \leqslant J_{\delta}(u, v)
$$

e

$$
J_{\delta}(u, v) \leqslant\left(k_{3}+\frac{\delta}{2}+\delta \gamma\right)\left[\|u\|_{H_{d}^{1}(\Omega)}^{2}+\|v\|_{L^{2}(\Omega)}^{2}+\|u\|_{L^{p}(\Omega)}^{p}\right]+k_{4},
$$

onde as constantes $k_{1}, k_{2}, k_{3}, k_{4}$ foram definidas na Proposição 4.1.3.

Demonstração: De fato, pela Proposição 4.1 .3 e usando a desigualdade $a b \leqslant \frac{a^{2}}{2}+\frac{b^{2}}{2}$, obtemos

$$
\begin{aligned}
J_{\delta}(u, v) & =I(u, v)+\delta \int_{\Omega}\left(u \cdot v+\gamma|u|^{2}\right) d x \leqslant k_{3}\left[\|u\|_{H_{d}^{1}(\Omega)}^{2}+\|v\|_{L^{2}(\Omega)}^{2}+\|u\|_{L^{p}(\Omega)}^{p}\right]+ \\
& +\frac{\delta\|u\|_{L^{2}(\Omega)}^{2}}{2}+\frac{\delta\|v\|_{L^{2}(\Omega)}^{2}}{2}+k_{4}+\delta \gamma\|u\|_{L^{2}(\Omega)}^{2} \\
& \leqslant\left(k_{3}+\frac{\delta}{2}+\delta \gamma\right)\left[\|v\|_{L^{2}(\Omega)}^{2}+\|u\|_{H_{d}^{1}(\Omega)}^{2}+\|u\|_{L^{p}(\Omega)}^{p}\right]+k_{4} .
\end{aligned}
$$

Além disso,

$$
\begin{aligned}
J_{\delta}(u, v) & =I(u, v)+\int_{\Omega}\left(u \cdot v+\gamma|u|^{2}\right) d x \geqslant k_{1}\left[\|u\|_{H_{d}^{1}(\Omega)}^{2}+\|v\|_{L^{2}(\Omega)}^{2}+\|u\|_{L^{p}(\Omega)}^{p}\right]-k_{2}- \\
& -\frac{\delta\|u\|_{L^{2}(\Omega)}^{2}}{2}-\frac{\delta\|v\|_{L^{2}(\Omega)}^{2}}{2} \geqslant\left(k_{1}-\frac{\delta}{2}\right)\left[\|u\|_{H_{d}^{1}(\Omega)}^{2}+\|v\|_{L^{2}(\Omega)}^{2}+\|u\|_{L^{p}(\Omega)}^{p}\right]-k_{2} .
\end{aligned}
$$

Corolário 4.1.5. Se u é uma solução fraca global de (4.10), então a função real 
$z(t) \doteq J_{\delta}\left(u(t), u_{t}(t)\right)$ é semicontínua inferiormente no intervalo $[0, T]$, para todo $T>0$.

Demonstração: Pela Proposição 4.1.2 basta verificarmos que a função $\int_{\Omega} \tilde{F}(u) d x$ é semicontínua inferiormente no intervalo $[0, T]$. De fato, por (4.14) decorre que $\Phi(u)=\left(\tilde{F}(u)+c_{3}\right)^{\frac{1}{p}} \in L^{\infty}\left(0, T ; L^{p}(\Omega)\right)$. Como $u \in C\left(0, T ; L^{2}(\Omega)\right)$ e $\left|\Phi\left(u_{1}\right)-\Phi\left(u_{2}\right)\right| \leqslant$ $K\left|u_{1}-u_{2}\right|$ temos que $\Phi \in C\left(0, T ; L^{2}(\Omega)\right)$ e do Teorema 1.1 .18 segue que $\Phi \in C_{w}\left(0, T ; L^{p}(\Omega)\right)$ e $\|\Phi(u)\|_{L^{p}(\Omega)}$ é semicontínua inferiormente em $[0, T]$. Portanto, a função

$$
\int_{\Omega} \tilde{F}(u) d x=\int_{\Omega} \Phi(u)^{p} d x-c_{3}|\Omega|=\|\Phi\|_{L^{p}(\Omega)}^{p}-c_{3}|\Omega|
$$

é semicontínua inferiormente no intervalo $[0, T]$.

Teorema 4.1.6. Se u $[0, \infty) \times \Omega \rightarrow \mathbb{R}$ é uma solução suficientemente regular de (4.10) $e$ $z(t)=J_{\delta}\left(u(t), \partial_{t} u(t)\right)$, então existem constantes $\mu_{\delta}>0$ e $\rho_{\delta}>0$ tais que

$$
\frac{d}{d t} z(t)+\mu_{\delta} z(t) \leqslant \rho_{\delta}
$$

e portanto,

$$
z(t) \leqslant z(0) e^{-\mu_{\delta} t}+\frac{\rho_{\delta}}{\mu_{\delta}}
$$

Demonstração: Tomando o produto na equação

$$
u_{t t}+2 \gamma u_{t}=d \Delta u-\tilde{f}(u)
$$

por $u_{t}$ e integrando em $\Omega$, obtemos

$$
\frac{1}{2} \frac{d}{d t} \int_{\Omega}\left[\left|u_{t}\right|^{2} d x+d|\nabla u|^{2}+2 \tilde{F}(u)\right] d x+2 \gamma \int_{\Omega}\left|u_{t}\right|^{2} d x=0
$$

Fazendo agora o produto da equação (4.29) por $u$ e integrando em $\Omega$, ficamos com

$$
\frac{d}{d t}\left[\int_{\Omega}\left(u \cdot u_{t}+\gamma|u|^{2}\right) d x\right]-\int_{\Omega}\left|u_{t}\right|^{2} d x+d \int_{\Omega}|\nabla u|^{2} d x+\int_{\Omega} \tilde{f}(u) \cdot u d x=0 .
$$

Somando (4.30) e a igualdade (4.31) multiplicada por $\delta$ e aplicando a desigualdade de Young (1.3) e a hipótese (4.16), podemos escrever

$$
\frac{d}{d t} J_{\delta}\left(u, u_{t}\right) \leqslant-2 \gamma \int_{\Omega}\left|u_{t}\right|^{2} d x+\delta \int_{\Omega}\left|u_{t}\right|^{2} d x-d \delta \int_{\Omega}|\nabla u|^{2} d x-\tilde{\gamma_{2}} \delta \int_{\Omega}|u|^{p} d x+\delta \tilde{c_{2}}|\Omega|+
$$




$$
\leqslant-\min \left\{2 \gamma-\delta, \delta, \frac{\delta \tilde{\gamma_{2}}}{2}\right\}\left[\left\|u_{t}\right\|_{L^{2}(\Omega)}^{2}+\|u\|_{H_{d}^{1}(\Omega)}^{2}+\|u\|_{L^{p}(\Omega)}^{p}\right]+\delta\left[\tilde{c_{2}}|\Omega|+\left(\frac{4}{\tilde{\gamma_{2} p}}\right)^{\frac{2}{p-2}}|\Omega| \frac{p-2}{p}\right] .
$$

De (4.32) e (4.26), obtemos

$$
\frac{d}{d t} J_{\delta}\left(u, u_{t}\right) \leqslant-k_{5} J_{\delta}\left(u, u_{t}\right)+k_{6}
$$

onde

$$
\begin{gathered}
k_{5}=\frac{\min \left\{2 \gamma-\delta, \delta, \delta \tilde{\gamma_{2}}\right\}}{k_{3}+\frac{\delta}{2}+\delta \gamma}, \\
k_{6}=k_{4} k_{5}+\delta\left[\tilde{c_{2}}|\Omega|+\left(\frac{4}{\tilde{\gamma_{2}} p}\right)^{\frac{2}{p-2}}|\Omega| \frac{p-2}{p}\right]
\end{gathered}
$$

e as constantes $k_{3}$ e $k_{4}$ foram dadas na Proposição 4.1.3.

E de (4.33) concluímos que

$$
z(t) \leqslant z(0) e^{-k_{5} t}+\frac{k_{6}}{k_{5}}
$$

ou seja, $\mu_{\delta}=k_{5}$ e $\rho_{\delta}=k_{6}$.

Fixemos $N>0$ e vamos definir o espaço de trajetórias $\mathcal{K}_{d}^{+}(N)$ da equação (4.10).

Definição 4.1.7. O espaço $\mathcal{K}_{d}^{+}(N)$ é o conjunto das soluções fracas globais u da equação (4.10) que verificam a desigualdade

$$
z(t) \leqslant N e^{-\mu_{\delta} t}+\frac{\rho_{\delta}}{\mu_{\delta}}
$$

para todo $t \geqslant 0$, onde $z(t)=J_{\delta}\left(u(t), \partial_{t} u(t)\right)$ e $\delta$ foi dado em (4.23).

Proposição 4.1.8. Se $u_{0} \in H_{d}^{1}(\Omega) \cap L^{p}(\Omega), u_{1} \in L^{2}(\Omega)$ e $z_{0}=J_{\delta}\left(u_{0}, u_{1}\right) \leqslant N$, então existe pelo menos uma solução fraca global $u \in \mathcal{K}_{d}^{+}(N)$ tal que

$$
\left.u\right|_{t=0}=u_{0},\left.\quad \partial_{t} u\right|_{t=0}=u_{1}
$$

Demonstração: Vamos construir uma solução $u \in \mathcal{K}_{d}^{+}(N)$ via método de Faedo-Galerkin. 
Do Teorema 1.1.29 sabemos que as autofunções $\left\{w_{j}\right\}_{j=0}^{\infty}$ do problema

$$
\left\{\begin{array}{l}
\Delta w+\lambda w=0 \quad \text { em } \Omega \\
\left.\frac{\partial w}{\partial \nu}\right|_{\Gamma}=0
\end{array}\right.
$$

constituem um sistema ortonormal completo para $L^{2}(\Omega)$. Além disso, $\left\{w_{j}\right\}_{j \in \mathbb{N}}$ é uma base de $H_{d}^{1}(\Omega) \cap L^{p}(\Omega)$. Isso decorre da suavidade de $\Omega$.

Consideremos o seguinte problema aproximado

$$
\partial_{t}^{2} u_{m}+2 \gamma \partial_{t} u_{m}=P_{m} d \Delta u_{m}-P_{m} \tilde{f}\left(u_{m}\right)
$$

com condições iniciais

$$
\left.u_{m}\right|_{t=0}=u_{0 m},\left.\quad \partial_{t} u_{m}\right|_{t=0}=u_{1 m},
$$

onde $P_{m}$ é o operador projeção ortogonal do $L^{2}(\Omega)$ sobre o subespaço gerado pelas $m+$ 1 primeiras autofunções $\left\{w_{0}, w_{1}(x), \ldots, w_{m}(x)\right\}$. Pelo Teorema de Carathéodory 1.1.21, o problema (4.34) possui uma solução local $u_{m}(t)=\sum_{j=1}^{m} a_{m j}(t) w_{j}(x)$ definida num intervalo $\left[0, t_{m}\right)$ com $t_{m}<T$.

Como $u_{0} \in H_{d}^{1}(\Omega) \cap L^{p}(\Omega)$ e $u_{1} \in L^{2}(\Omega)$, podemos aproximá-los por combinações lineares finitas dos $w_{j}$ de modo que $u_{0 m} \rightarrow u_{0}$ fortemente em $H_{d}^{1}(\Omega) \cap L^{p}(\Omega)$ e $u_{1 m} \rightarrow u_{1}$ fortemente em $L^{2}(\Omega)$. Por (4.14), (4.16) e (4.24) resulta que

$$
z_{m}(0)=J_{\delta}\left(u_{0 m}, u_{1 m}\right) \rightarrow J_{\delta}\left(u_{0}, u_{1}\right)=z_{0}
$$

quando $m \rightarrow \infty$.

Como a solução aproximada $u_{m}$ satisfaz a desigualdade diferencial (4.27), temos que

$$
z_{m}(t) \leqslant z_{m}(0) e^{-\mu_{\delta} t}+\frac{\rho_{\delta}}{\mu_{\delta}}
$$

para todo $t \geqslant 0$.

Usando a Proposição 1.1.39 e as estimativas (4.18), (4.25), (4.26) e (4.36) concluímos que para todo $T>0$

(i) a sequência $\left\{u_{m}\right\}$ é limitada em $L^{\infty}\left(0, T ; L^{p}(\Omega)\right) \cap L^{\infty}\left(0, T ; H_{d}^{1}(\Omega)\right)$;

(ii) $\left\{\partial_{t} u_{m}\right\}$ é limitada em $L^{\infty}\left(0, T ; L^{2}(\Omega)\right)$;

(iii) $\left\{\tilde{f}\left(u_{m}\right)\right\}$ é limitada em $L^{\infty}\left(\mathbb{R}_{+} ; L^{q}(\Omega)\right)$;

(iv) $\left\{\left(\tilde{F}\left(u_{m}\right)+c_{3}\right)^{\frac{1}{p}}\right\}$ é limitada em $L^{\infty}\left(\mathbb{R}_{+} ; L^{p}(\Omega)\right)$;

(v) $\left\{\partial_{t}^{2} u_{m}\right\}$ é limitada em $L^{\infty}\left(\mathbb{R}_{+} ; H^{-r}(\Omega)\right)$ e

(vi) $\left\{\frac{\partial u_{m}}{\partial \nu}\right\}$ é limitada em $L^{\infty}\left(0, T ; H^{-\frac{1}{2}}(\Gamma)\right)$. 
Em virtude do Teorema de Compacidade de Alaoglu 1.1.24 e do Corolário 1.1.25 podemos passar a uma subsequência, indicada por $u_{m}$, que converge fracamente para uma função $u$ satisfazendo $u \in L^{\infty}\left(0, T ; L^{p}(\Omega)\right) \cap L^{\infty}\left(0, T ; H_{d}^{1}(\Omega)\right), \partial_{t} u \in L^{\infty}\left(0, T ; L^{2}(\Omega)\right), \partial_{t}^{2} u \in$ $L^{\infty}\left(0, T ; H^{-r}(\Omega)\right)$ e tal que

(1) $u_{m} \stackrel{*}{\rightarrow} u$ em $L^{\infty}\left(0, T ; L^{p}(\Omega)\right) \cap L^{\infty}\left(0, T ; H_{d}^{1}(\Omega)\right)$

(2) $\partial_{t} u_{m} \stackrel{*}{\rightarrow} \partial_{t} u$ em $L^{\infty}\left(0, T ; L^{2}(\Omega)\right)$,

(3) $\tilde{f}\left(u_{m}\right) \stackrel{*}{\rightarrow} \tilde{f}(u)$ em $L^{\infty}\left(0, T ; L^{q}(\Omega)\right)$,

(4) $\left(\tilde{F}\left(u_{m}\right)+c_{3}\right)^{\frac{1}{p}} \stackrel{*}{\rightarrow}\left(\tilde{F}(u)+c_{3}\right)^{\frac{1}{p}}$ em $L^{\infty}\left(0, T ; L^{p}(\Omega)\right)$,

(5) $\partial_{t}^{2} u_{m} \stackrel{*}{\rightarrow} \partial_{t}^{2} u$ em $L^{\infty}\left(0, T ; H^{-r}(\Omega)\right)$,

(6) $\frac{\partial u_{m}}{\partial \nu} \stackrel{*}{\rightarrow} \frac{\partial u}{\partial \nu}$ em $L^{\infty}\left(0, T ; H^{-\frac{1}{2}}(\Gamma)\right)$,

para todo intervalo $[0, T] \subset \mathbb{R}_{+}$.

Tomando o limite na equação (4.34) encontramos que $u$ verifica

$$
\partial_{t}^{2} u+2 \gamma \partial_{t} u=d \Delta u-\tilde{f}(u)
$$

Agora, para demonstrar que $u \in \mathcal{K}^{+}(N)$ devemos checar que

$$
z(t) \leqslant z(0) e^{\mu_{\delta} t}+\frac{\rho_{\delta}}{\mu_{\delta}}
$$

para todo $t \geqslant 0$.

Afirmamos que

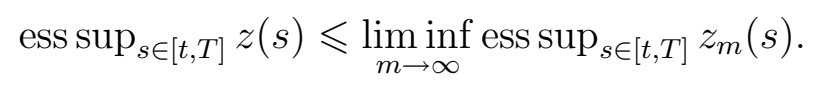

De fato, das convergências fracas (1)-(5) acima, decorre que

$$
\operatorname{ess~sup}_{s \in[t, T]} \int_{\Omega}\left(\tilde{F}(u)+c_{3}\right) d x \leqslant \liminf _{m \rightarrow \infty} \operatorname{ess}_{\sup _{s \in[t, T]}} \int_{\Omega}\left(\tilde{F}\left(u_{m}\right)+c_{3}\right) d x
$$

Analogamente,

$$
\begin{aligned}
& \operatorname{ess~sup}_{s \in[t, T]}\left(\|u(s)\|_{H_{d}^{1}(\Omega)}^{2}+\left\|\partial_{t} u(s)\right\|_{L^{2}(\Omega)}^{2}\right) \leqslant \\
& \liminf _{m \rightarrow \infty} \operatorname{ess~sup}_{s \in[t, T]}\left(\left\|u_{m}(s)\right\|_{H_{d}^{1}(\Omega)}^{2}+\left\|\partial_{t} u_{m}(s)\right\|_{L^{2}(\Omega)}^{2}\right) .
\end{aligned}
$$

De (4.35) e (4.36), obtemos

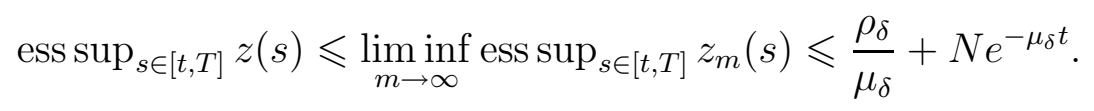


Como $z(s)$ é semicontínua inferior, concluímos que

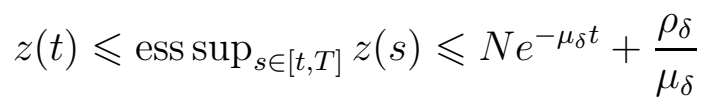

e portanto $u \in \mathcal{K}^{+}(N)$.

Vamos verificar os dados iniciais.

Sabemos que

$$
\int_{0}^{T}\left\langle u_{m}(t), w(t)\right\rangle d t \rightarrow \int_{0}^{T}\langle u(t), w(t)\rangle d t
$$

para todo $w \in L^{1}\left(0, T ;\left(L^{p}(\Omega) \cap H_{d}^{1}(\Omega)\right)^{\prime}\right) ; \mathrm{e}$

$$
\int_{0}^{T}\left\langle u_{m}^{\prime}(t), w(t)\right\rangle d t \rightarrow \int_{0}^{T}\left\langle u^{\prime}(t), w(t)\right\rangle d t
$$

para todo $w \in L^{1}\left(0, T ; L^{2}(\Omega)\right)$.

Seja $\psi \in C^{1}([0, T])$ uma função satisfazendo $\psi(0)=1$ e $\psi(T)=0$. Tome $w_{1} \in$ $H_{d}^{1}(\Omega) \cap L^{p}(\Omega)$, de $(4.38)$ e $(4.39)$, resulta que

$$
\int_{0}^{T}\left\langle u_{m}(t), \psi^{\prime}(t) w_{1}\right\rangle d t \rightarrow \int_{0}^{T}\left\langle u(t), \psi^{\prime}(t) w_{1}\right\rangle d t
$$

e

$$
\int_{0}^{T}\left\langle u_{m}^{\prime}(t), \psi(t) w_{1}\right\rangle d t \rightarrow \int_{0}^{T}\left\langle u^{\prime}(t), \psi(t) w_{1}\right\rangle d t
$$

Somando (4.40) e (4.41) ficamos com

$$
\int_{0}^{T}\left\langle u_{m}(t), \psi^{\prime}(t) w_{1}\right\rangle d t+\int_{0}^{T}\left\langle u_{m}^{\prime}(t), \psi(t) w_{1}\right\rangle d t \rightarrow \int_{0}^{T}\left\langle u(t), \psi^{\prime}(t) w_{1}\right\rangle d t+\int_{0}^{T}\left\langle u^{\prime}(t), \psi(t) w_{1}\right\rangle d t .
$$

Como

$$
\frac{d}{d t}\left\langle u_{m}(t), \psi(t) w_{1}\right\rangle=\left\langle u_{m}^{\prime}(t), \psi(t) w_{1}\right\rangle+\left\langle u_{m}(t), \psi^{\prime}(t) w_{1}\right\rangle
$$

decorre que

$$
\int_{0}^{T} \frac{d}{d t}\left\langle u_{m}(t), \psi(t) w_{1}\right\rangle d t \rightarrow \int_{0}^{T} \frac{d}{d t}\left\langle u(t), \psi(t) w_{1}\right\rangle d t
$$


donde

$$
-\left\langle u_{m}(0), w_{1}\right\rangle \rightarrow-\left\langle u(0), w_{1}\right\rangle
$$

para todo $w_{1} \in H_{d}^{1}(\Omega) \cap L^{p}(\Omega)$.

Em razão de $u_{m}(0)=u_{0 m} \rightarrow u_{0}$ em $H_{d}^{1}(\Omega) \cap L^{p}(\Omega)$ e $H_{d}^{1}(\Omega) \cap L^{p}(\Omega) \hookrightarrow L^{2}(\Omega)$, resulta que $\left\langle u_{0 m}, \eta\right\rangle \rightarrow\left\langle u_{0}, \eta\right\rangle$, para todo $\eta \in L^{2}(\Omega)$.

E pela unicidade do limite fraco temos que $\left\langle u(0), w_{1}\right\rangle=\left\langle u_{0}, w_{1}\right\rangle$, para todo $w_{1} \in$ $H_{d}^{1}(\Omega) \cap L^{p}(\Omega)$.

Portanto, $u(0)=u_{0}$.

Agora, de (4.34) podemos escrever

$$
\left\langle u_{m}^{\prime \prime}(t), w(t)\right\rangle+2 \gamma\left\langle u_{m}^{\prime}(t), w(t)\right\rangle+\left\langle\nabla u_{m}(t), \nabla w(t)\right\rangle+\left\langle\tilde{f}\left(u_{m}(t)\right), w(t)\right\rangle=0
$$

para todo $w \in H_{d}^{1}(\Omega) \cap L^{p}(\Omega)$.

Fazendo o produto da equação aproximada (4.43) por $\psi$ e integrando por partes, obtemos

$$
\begin{aligned}
& -\int_{0}^{T}\left\langle u_{m}^{\prime}(t), w(t)\right\rangle \psi^{\prime}(t) d t-\left\langle u_{1 m}, w(t)\right\rangle+2 \gamma \int_{0}^{T}\left\langle u_{m}^{\prime}(t), w(t)\right\rangle \psi(t) d t+ \\
& +\int_{0}^{T}\left\langle\nabla u_{m}(t), \nabla w(t)\right\rangle \psi(t) d t+\int_{0}^{T}\left\langle\tilde{f}\left(u_{m}(t)\right), w(t)\right\rangle \psi=0 .
\end{aligned}
$$

Como $\psi, \psi^{\prime} \in L^{2}([0, T])$ e $u_{1 m} \rightarrow u_{1}$ em $L^{2}(\Omega)$, tomando o limite na igualdade (4.44), temos que

$$
\begin{aligned}
& -\left\langle u_{1}, w\right\rangle-\int_{0}^{T}\left\langle u^{\prime}(t), w(t)\right\rangle \psi^{\prime}(t) d t+2 \gamma \int_{0}^{T}\left\langle u^{\prime}(t), w(t)\right\rangle \psi(t) d t+ \\
& +\int_{0}^{T}\langle\nabla u(t), \nabla w(t)\rangle \psi(t) d t+\int_{0}^{T}\langle\tilde{f}(u), w(t)\rangle \psi(t)=0 .
\end{aligned}
$$

E novamente por integração por partes, decorre que

$$
\begin{aligned}
& -\left\langle u_{1}, w(t)\right\rangle-\int_{0}^{T}\left\langle u^{\prime}(t), w(t)\right\rangle+\int_{0}^{T} \frac{d}{d t}\left\langle u^{\prime}(t), w(t)\right\rangle \psi(t) d t+2 \gamma \int_{0}^{T}\left\langle u^{\prime}(t), w(t)\right\rangle d t+ \\
& +\int_{0}^{T}\langle\nabla u(t), \nabla w(t)\rangle \psi(t) d t+\int_{0}^{T}\langle\tilde{f}(u), w(t)\rangle \psi(t)=0 .
\end{aligned}
$$


De (4.37) podemos escrever

$$
\left\langle\partial_{t}^{2} u, w\right\rangle+2 \gamma\left\langle\partial_{t} u, w\right\rangle+\langle\nabla u, \nabla w\rangle+\langle\tilde{f}(u), w\rangle=0
$$

para todo $w \in H_{d}^{1}(\Omega) \cap L^{p}(\Omega)$, em $\mathcal{D}^{\prime}(0, T)$.

Usando (4.45) ficamos com $\left\langle u_{1}, w\right\rangle=\left\langle u^{\prime}(0), w\right\rangle$, para todo $w \in H_{d}^{1}(\Omega) \cap L^{p}(\Omega)$. E por densidade, concluímos que $u_{1}=u^{\prime}(0)$.

Vamos definir os conjuntos $\mathcal{F}_{l o c, d}^{+}, \mathcal{F}_{b, d}^{+}$e a topologia $\Theta_{l o c, d}^{+}$associados à equação (4.10).

Começamos definindo o conjunto $\mathcal{F}_{T, d}$ da seguinte forma

$$
\begin{aligned}
\mathcal{F}_{T, d}= & \left\{v: v \in L^{\infty}\left(0, T ; L^{p}(\Omega)\right) \cap L^{\infty}\left(0, T ; H_{d}^{1}(\Omega)\right), \partial_{t} v \in L^{\infty}\left(0, T ; L^{2}(\Omega)\right),\right. \\
& \left.\partial_{t}^{2} v \in L^{\infty}\left(0, T ; H^{-r}(\Omega)\right)\right\}
\end{aligned}
$$

e o munimos da topologia $\Theta_{T, d}$ que, em termos de sequências, é descrita da seguinte maneira: uma sequência $\left\{v_{m}\right\} \subset \mathcal{F}_{T, d}$ converge para uma função $v \in \mathcal{F}_{T, d}$ em $\Theta_{T, d}$ se $v_{m} \stackrel{*}{\rightarrow} v$ em $L^{\infty}\left(0, T ; H_{d}^{1}(\Omega)\right) \cap L^{\infty}\left(0, T ; L^{p}(\Omega)\right), \partial_{t} v_{m} \stackrel{*}{\rightarrow} \partial_{t} v$ em $L^{\infty}\left(0, T ; L^{2}(\Omega)\right)$ e $\partial_{t}^{2} v_{m} \stackrel{*}{\rightarrow} \partial_{t}^{2} v$ em $L^{\infty}\left(0, T ; H^{-r}(\Omega)\right)$.

Como já observamos na Seção 3.1 uma topologia definida da forma acima torna $\mathcal{F}_{T, d}$ um espaço topológico de Hausdorff e Frechet-Uryshon com base topológica enumerável.

O conjunto $\mathcal{F}_{T, d}$ e a topologia $\Theta_{T, d}$ dão origem aos conjuntos $\mathcal{F}_{l o c, d}^{+}, \mathcal{F}_{b, d}^{+}$e a topologia $\Theta_{l o c, d}^{+}$

Temos que

$$
\begin{aligned}
\mathcal{F}_{l o c, d}^{+}= & \left\{v: v \in L_{l o c}^{\infty}\left(\mathbb{R}_{+} ; L^{p}(\Omega)\right) \cap L_{l o c}^{\infty}\left(\mathbb{R}_{+} ; H_{d}^{1}(\Omega)\right), \partial_{t} v \in L_{l o c}^{\infty}\left(\mathbb{R}_{+} ; L^{2}(\Omega)\right) \mathrm{e}\right. \\
& \left.\partial_{t}^{2} v \in L_{l o c}^{\infty}\left(\mathbb{R}_{+} ; H^{-r}(\Omega)\right)\right\} ; \\
\mathcal{F}_{b, d}^{+}= & \left\{v \in \mathcal{F}_{l o c, d}^{+}: v \in L^{\infty}\left(\mathbb{R}_{+} ; L^{p}(\Omega)\right) \cap L^{\infty}\left(\mathbb{R}_{+} ; H_{d}^{1}(\Omega)\right) \cap \partial_{t} v \in L^{\infty}\left(\mathbb{R}_{+} ; L^{2}(\Omega)\right) \mathrm{e}\right. \\
& \left.\partial_{t}^{2} v \in L^{\infty}\left(\mathbb{R}_{+} ; H^{-r}(\Omega)\right) \operatorname{com}\|v\|_{\mathcal{F}_{b, d}^{+}}<+\infty\right\},
\end{aligned}
$$

onde

$$
\|v\|_{\mathcal{F}_{b, d}^{+}}=\|v\|_{L^{\infty}\left(\mathbb{R}_{+} ; L^{p}(\Omega)\right)}+\|v\|_{L^{\infty}\left(\mathbb{R}_{+} ; H_{d}^{1}(\Omega)\right)}+\left\|\partial_{t} v\right\|_{L^{\infty}\left(\mathbb{R}_{+} ; L^{2}(\Omega)\right)}+\left\|\partial_{t}^{2} v\right\|_{L^{\infty}\left(\mathbb{R}_{+} ; H^{-r}(\Omega)\right)}
$$

E a topologia $\Theta_{l o c, d}^{+}$em $\mathcal{F}_{l o c, d}^{+}$é definida da seguinte forma: $v_{m} \rightarrow v$ em $\Theta_{l o c, d}^{+}$quando $\Pi_{[0, T]} v_{m} \rightarrow \Pi_{[0, T]} v$ em $\Theta_{T, d}$ para todo $T>0$.

O espaço $\mathcal{F}_{l o c, d}^{+}$munido da topologia $\Theta_{l o c, d}^{+}$é um espaço topológico Hausdorff, Frechet- 
Uryshon e possui uma base enumerável. De fato, tomemos $z \in \mathcal{F}_{l o c, d}^{+}$e indiquemos por $\tau_{1}$ : a topologia gerada pela norma do espaço $\left(L^{\infty}\left(0, T ; H_{d}^{1}(\Omega)\right),\|\cdot\|_{1}\right)$;

$\tau_{2}$ : a topologia em $\left(L^{\infty}\left(0, T ; L^{p}(\Omega)\right),\|\cdot\|_{2}\right)$;

$\tau_{3}$ : a topologia em $\left(L^{\infty}\left(0, T ; L^{2}(\Omega)\right),\|\cdot\|_{3}\right)$ e

$\tau_{4}$ : a topologia em $\left(L^{\infty}\left(0, T ; H^{-r}(\Omega)\right),\|\cdot\|_{4}\right)$.

Consideremos $\left\{B_{1}\left(z, \frac{1}{n}\right): n \in \mathbb{N}\right\},\left\{B_{2}\left(z, \frac{1}{n}\right): n \in \mathbb{N}\right\},\left\{B_{3}\left(\partial_{t} z, \frac{1}{n}\right): n \in \mathbb{N}\right\} \mathrm{e}$ $\left\{B_{4}\left(\partial_{t}^{2} z, \frac{1}{n}\right): n \in \mathbb{N}\right\}$, onde $B_{i}\left(y, \frac{1}{n}\right)=\left\{\tilde{y}:\|y-\tilde{y}\|_{i}<\frac{1}{n}\right\}$ para $i=1,2,3$, 4. Cada um desses conjuntos constitui uma coleção enumerável de vizinhanças abertas de $z$ nas topologias $\tau_{1}$, $\tau_{2}$, respectivamente, de $\partial_{t} z$ na topologia $\tau_{3}$ e de $\partial_{t}^{2} z$ na topologia $\tau_{4}$. Façamos a reunião desses conjuntos abertos $\cup_{j=1}^{2} B_{j}\left(z, \frac{1}{n}\right) \cup B_{3}\left(\partial_{t} z, \frac{1}{n}\right) \cup B_{4}\left(\partial_{t}^{2} z, \frac{1}{n}\right)=\mathfrak{F}(z)$. Observemos que essa reunião é enumerável, ou seja, podemos considerar $\mathfrak{F}(z)=\left\{F_{n}: n \in \mathbb{N}\right\}$.

Seja $I \subset \mathbb{N}$ um subconjunto finito tal que $\left\{z, \partial_{t} z\right\} \subset V_{I}=\left(\cap_{n \in I} F_{n}^{z}\right) \cup\left(\cap_{n \in I} F_{n}^{\partial_{t} z}\right) \cup$ $\left(\cap_{n \in I} F_{n}^{\partial_{t}^{2} z}\right)$, onde $F_{n}^{z}$ denota os elementos de $\mathfrak{F}(z)$ contendo $z, F_{n}^{\partial_{t} z}$ são os elementos de $\mathfrak{F}(z)$ contendo $\partial_{t} z$ e $F_{n}^{\partial_{t} z}$ são os elementos de $\mathfrak{F}(z)$ contendo $\partial_{t}^{2} z$. Definamos $\tilde{B}(z)=\left\{V_{I}: I \subset\right.$ $\mathbb{N}, I$ é finito $\}$. Este conjunto é um elemento básico da topologia $\Theta_{l o c, d}^{+}$contendo z. Logo, a topologia $\Theta_{l o c, d}^{+}$é gerada pela reunião dos conjuntos da forma $\cup_{z \in \mathcal{F}_{l o c, d}^{+}} \tilde{B}(z)$.

Se $z_{n} \rightarrow z$ em $\Theta_{l o c, d}^{+}$, segue da Proposição 1.2.9 que o espaço topológico $\mathcal{F}_{l o c, d}^{+}$munido da topologia $\Theta_{l o c, d}^{+}$é Hausdorff. Além disso, como existe uma base enumerável local para todo elemento de $\mathcal{F}_{l o c, d}^{+}$este espaço é Frechet-Uryshon.

O espaço $\mathcal{K}_{d}^{+}(N)$ munido da topologia $\Theta_{l o c, d}^{+}$é o espaço de trajetórias da equação

Definamos a aplicação $T(t): \mathcal{F}_{l o c, d}^{+} \rightarrow \mathcal{F}_{l o c, d}^{+}$por $T(t) u(s)=u(t+s)$ e consideremos a família $\{T(t): t \geqslant 0\}$ que constitui um semigrupo. Como a equação (4.10) é autônoma temos que $\mathcal{K}_{d}^{+}(N)$ é invariante pelo semigrupo $\{T(t): t \geqslant 0\}$, logo podemos considerar $T(t): \mathcal{K}_{d}^{+}(N) \rightarrow \mathcal{K}_{d}^{+}(N)$.

Proposição 4.1.9. Se $\mathcal{K}_{d}^{+}(N)$ é o espaço de trajetórias da equação (4.10), então para toda função $u \in \mathcal{K}_{d}^{+}(N)$ temos que $T(t) u \in \mathcal{K}_{d}^{+}(N)$ para $t \geqslant 0$.

Demonstração: Se $u(\cdot) \in \mathcal{K}_{d}^{+}(N)$, então $u(t+\cdot)$ é solução fraca de $(4.10)$, pois a equação é autônoma. Sabemos que a função $u$ satisfaz

$$
z(s) \leqslant N e^{-\mu_{\delta} s}+\frac{\rho_{\delta}}{\mu_{\delta}},
$$

para $s \geqslant 0, \log O$

$$
T(t) z(s)=z(t+s) \leqslant N e^{-\mu_{\delta}(t+s)}+\frac{\rho_{\delta}}{\mu_{\delta}} \leqslant N e^{-\mu_{\delta} t}+\frac{\rho_{\delta}}{\mu_{\delta}}
$$

para $s \geqslant 0$ e $t \geqslant 0$, ou seja, $T(t) u \in \mathcal{K}_{d}^{+}(N)$. 
Proposição 4.1.10. Se $\mathcal{K}_{d}^{+}(N)$ é o espaço de trajetórias da equação $(4.10)$, então $\mathcal{K}_{d}^{+}(N) \subset$ $\mathcal{F}_{b, d}^{+}$.

Demonstração: A demonstração decorre do fato que

$$
z(t) \leqslant e^{-\mu_{\delta} t} N+\frac{\rho_{\delta}}{\mu_{\delta}},
$$

para todo $t \geqslant 0$. De fato, se $u \in \mathcal{K}_{d}^{+}(N)$, então de (4.25) e (4.46), resulta que

$$
\left(k_{1}-\frac{\delta}{2}\right)\left[\|u\|_{H_{d}^{1}(\Omega)}^{2}+\left\|u_{t}\right\|_{L^{2}(\Omega)}^{2}+\|u\|_{L^{p}(\Omega)}^{p}\right]-k_{2} \leqslant z(t) \leqslant \frac{\rho_{\delta}}{\mu_{\delta}}+N,
$$

para todo $t \geqslant 0$. Para obter (4.47) tomamos $h=t-s$ em (4.46) e fazemos $s \rightarrow+\infty$. Lembremos que $k_{1}=\frac{1}{2} \min \left\{1, \tilde{\gamma}_{1}\right\}$ e $k_{2}=\tilde{c_{1}}|\Omega|+\frac{1}{2}\left(\frac{2}{\tilde{\gamma_{1} p}}\right)^{\frac{2}{p-2}}|\Omega|\left(\frac{p-2}{p}\right)$.

Da equação (4.10) e da estimativa (4.18) decorre que $\partial_{t}^{2} u \in L^{\infty}\left(\mathbb{R}_{+} ; H^{-r}(\Omega)\right)$. Portanto $\mathcal{K}_{d}^{+}(N) \subset \mathcal{F}_{b, d}^{+}$para todo $N>0$.

Proposição 4.1.11. O espaço de trajetórias $\mathcal{K}_{d}^{+}(N)$ é fechado na topologia $\Theta_{l o c, d}^{+}$.

Demonstração: Seja $\left\{u_{m}\right\}_{m \in \mathbb{N}} \subset \mathcal{K}_{d}^{+}(N)$ uma sequência tal que

$$
u_{m} \rightarrow u \quad \mathrm{em} \Theta_{l o c, d}^{+}
$$

De (4.48) e da imersão $H^{1}\left(0, T ; L^{2}(\Omega)\right) \stackrel{c}{\hookrightarrow} L^{2}\left(0, T ; L^{2}(\Omega)\right)$, passando a uma subsequência, indicada por $u_{m}$, temos que $u_{m}(s, x) \rightarrow u(s, x)$ para quase todo $(s, x) \in \mathbb{R}_{+} \times \Omega$. A continuidade da função $\tilde{f}$ implica que $\tilde{f}\left(u_{m}(s, x)\right) \rightarrow \tilde{f}(u(s, x))$ para quase todo $(s, x) \in \mathbb{R}_{+} \times \Omega$. Por outro lado, de (4.18) segue que $\left\{\tilde{f}\left(u_{m}\right)\right\}$ é limitada em $L^{q}\left(0, T ; L^{q}(\Omega)\right)$. Aplicando o Lema de Lions 1.1.17, concluímos que $\tilde{f}\left(u_{m}\right) \rightarrow \tilde{f}(u)$ em $L^{q}\left(0, T ; L^{q}(\Omega)\right)$.

Como $u_{m}$ satisfaz a equação

$$
\partial_{t}^{2} u_{m}+2 \gamma \partial_{t} u_{m}=d \Delta u_{m}-\tilde{f}\left(u_{m}\right),
$$

tomando o limite quando $m \rightarrow+\infty$ em (4.49) deduzimos que a função $u$ é uma solução fraca da equação (4.10).

Como

$$
z_{m}(t)=J_{\delta}\left(u_{m}, \partial_{t} u_{m}\right) \leqslant N e^{-\mu_{\delta} t}+\frac{\rho_{\delta}}{\mu_{\delta}}
$$


para todo $t \geqslant 0$, fazendo $m \rightarrow+\infty$ em ambos os lados dessa desigualdade, obtemos

$$
z(t)=J_{\delta}\left(u, \partial_{t} u\right) \leqslant N e^{-\mu_{\delta} t}+\frac{\rho_{\delta}}{\mu_{\delta}}
$$

para todo $t \geqslant 0$. Portanto $u \in \mathcal{K}_{d}^{+}(N)$, donde concluímos que $\mathcal{K}_{d}^{+}(N)$ é fechado com relação a topologia $\Theta_{l o c, d}^{+}$.

Agora, fixemos um valor $\delta=\delta_{0}$ verificando (4.23). Vamos definir o conjunto

$$
P=\left\{u \in \mathcal{F}_{b, d}^{+}: z(t)=J_{\delta_{0}}\left(u, \partial_{t} u\right) \leqslant 2 \frac{\rho_{\delta_{0}}}{\mu_{\delta_{0}}}\right\} .
$$

Observemos que o conjunto $P$ é tal que $\|u\|_{\mathcal{F}_{b, d}^{+}}<+\infty$ para toda função $u \in \mathcal{F}_{b, d}^{+}$e é compacto em $\Theta_{l o c, d}^{+}$. Além disso, a desigualdade (4.28) implica que o conjunto $P$ é um conjunto absorvente do espaço $\mathcal{K}_{d}^{+}(N)$. De fato, seja $B \subset \mathcal{K}_{d}^{+}(N)$ um conjunto de trajetórias de (4.10). Tomando $t_{0}$ tal que $N e^{-\mu_{\delta_{0}} t_{0}} \leqslant \frac{\rho_{\delta_{0}}}{\mu_{\delta_{0}}}$ temos que $T(t) B \subset P$ para todo $t \geqslant t_{0}$.

Proposição 4.1.12. Se $\tilde{f}$ satisfaz (4.14)-(4.16), então para cada d > 0 a equação (4.10) possui um atrator de trajetórias $\mathfrak{A}_{d}(N)$.

Demonstração: Como o semigrupo translação $T(t): \mathcal{K}_{d}^{+}(N) \rightarrow \mathcal{K}_{d}^{+}(N)$ é contínuo e o conjunto $P \cap \mathcal{K}_{d}^{+}(N)$ é atrativo concluímos pelo Teorema 1.3 .10 que existe um atrator de trajetórias $\mathfrak{A}_{d}(N)$ para a equação (4.10).

Definamos os conjuntos

$$
\begin{aligned}
\mathcal{F}_{l o c, d}= & \left\{v: v \in L_{l o c}^{\infty}\left(\mathbb{R} ; L^{p}(\Omega)\right) \cap L_{l o c}^{\infty}\left(\mathbb{R} ; H_{d}^{1}(\Omega)\right), \partial_{t} v \in L_{l o c}^{\infty}\left(\mathbb{R} ; L^{2}(\Omega)\right) \mathrm{e}\right. \\
& \left.\partial_{t}^{2} v \in L_{l o c}^{\infty}\left(\mathbb{R} ; H^{-r}(\Omega)\right)\right\} ; \\
\mathcal{F}_{b, d}= & \left\{v: v \in L^{\infty}\left(\mathbb{R} ; L^{p}(\Omega)\right) \cap L^{\infty}\left(\mathbb{R} ; H_{d}^{1}(\Omega)\right) \cap \partial_{t} v \in L^{\infty}\left(\mathbb{R} ; L^{2}(\Omega)\right) \mathrm{e}\right. \\
& \left.\partial_{t}^{2} v \in L^{\infty}\left(\mathbb{R} ; H^{-r}(\Omega)\right) \operatorname{com}\|v\|_{\mathcal{F}_{b, d}}<+\infty\right\},
\end{aligned}
$$

onde

$$
\|v\|_{\mathcal{F}_{b, d}}=\|v\|_{L^{\infty}\left(\mathbb{R} ; L^{p}(\Omega)\right)}+\|v\|_{L^{\infty}\left(\mathbb{R} ; H_{d}^{1}(\Omega)\right)}+\left\|\partial_{t} v\right\|_{L^{\infty}\left(\mathbb{R} ; L^{2}(\Omega)\right)}+\left\|\partial_{t}^{2} v\right\|_{L^{\infty}\left(\mathbb{R} ; H^{-r}(\Omega)\right)}
$$

E munimos $\mathcal{F}_{l o c, d}$ com a topologia $\Theta_{l o c, d}$ definida da seguinte forma: $v_{m} \rightarrow v$ em $\Theta_{l o c, d}$ quando $\Pi_{[-T, T]} v_{m} \rightarrow \Pi_{[-T, T]} v$ em $\Theta_{[-T, T], d}$ para todo $T>0$.

Denotamos por $\mathcal{K}_{d}$ o núcleo da equação (4.10) consistindo de todas as soluções fracas 
$u$ da equação

$$
\partial_{t}^{2} u+2 \gamma \partial_{t} u=d \Delta u-f(u)+|u|^{\alpha-1} u, \quad t \in \mathbb{R},
$$

limitadas na norma do espaço $\mathcal{F}_{b, d}$ e satisfazendo a desigualdade

$$
z(t) \leqslant J_{\delta}\left(u(t), u_{t}(t)\right) \leqslant \frac{\rho_{\delta}}{\mu_{\delta}}
$$

para todo $t \in \mathbb{R}$.

Para obter (4.50) basta observar que uma trajetória completa $u$ da equação (4.10) satisfaz $\Pi_{+} u(\cdot+h) \in \mathcal{K}_{d}^{+}(N)$ para todo $h \in \mathbb{R}$ e portanto,

$$
z(s+h) \leqslant \frac{\rho_{\delta}}{\mu_{\delta}}+N e^{-\mu_{\delta} s}
$$

para todo $s \geqslant 0$. Tomando $h=t-s$ e fazendo $s \rightarrow+\infty$ obtemos (4.50).

Observemos que fixando $\delta=\delta_{0}$, temos que $z(t) \leqslant R_{\delta_{0}}$ para todo $t \in \mathbb{R}$ e a trajetória completa $u$ é uniformemente limitada na norma do espaço $\mathcal{F}_{b, d}$. Além disso, o núcleo $\mathcal{K}_{d}$ independe de $N$.

Analogamente ao Teorema 3.1.9 temos o seguinte resultado

Teorema 4.1.13. Seja $\mathcal{K}_{d}^{+}(N)$ o espaço de trajetórias da equação (4.10) para $N>0$ fixado. $S e \mathfrak{A}_{d}(N) \subset P$ é o atrator de trajetórias do semigrupo translação $T(t): \mathcal{K}_{d}^{+}(N) \rightarrow \mathcal{K}_{d}^{+}(N)$, então o conjunto $\mathfrak{A}_{d}(N)$ é limitado na norma do espaço $\mathcal{F}_{b, d}^{+}$e é compacto em relação a topologia $\Theta_{l o c, d}^{+}$. Além disso,

$$
\mathfrak{A}_{d}=\Pi_{+} \mathcal{K}_{d}
$$

$O$ conjunto $\mathcal{K}_{d}$ é limitado em $\mathcal{F}_{b, d}$ e compacto em $\Theta_{\text {loc,d }}$.

Demonstração: A igualdade (4.51) é verificada como na demonstração do Teorema 3.1.9.

Proposição 4.1.14. o atrator de trajetórias $\mathfrak{A}_{d}(N)$ não depende de $N>0$, isto é, $\mathfrak{A}_{d}(N) \equiv$ $\mathfrak{A}_{d}$.

Demonstração: De fato, seja $N>0$ então $\mathcal{K}_{d}^{+}(N) \subseteq \mathcal{K}_{d}^{+}\left(N_{1}\right)$ para todo $N_{1} \geqslant N$. Logo, $\mathfrak{A}_{d}(N) \subseteq \mathfrak{A}_{d}\left(N_{1}\right)$ para $N_{1} \geqslant N$.

Como $T(t) \mathcal{K}_{d}^{+}\left(N_{1}\right) \subseteq \mathcal{K}_{d}^{+}(N)$ para $t \geqslant \frac{1}{\mu_{\delta}} \ln \left(\frac{N_{1}}{N}\right)$ temos, em particular, que $T(t) \mathfrak{A}_{d}\left(N_{1}\right) \subseteq$ $\mathcal{K}_{d}^{+}(N)$ para $t \geqslant \frac{1}{\mu_{\delta}} \ln \left(\frac{N_{1}}{N}\right)$. Mas sabemos que o conjunto $\mathfrak{A}_{d}\left(N_{1}\right)$ é estritamente invariante, 
isto é, $\mathfrak{A}_{d}\left(N_{1}\right)=T(t) \mathfrak{A}_{d}\left(N_{1}\right)$, donde $\mathfrak{A}_{d}\left(N_{1}\right) \subseteq \mathcal{K}_{d}^{+}(N)$ e sendo $\mathfrak{A}_{d}(N)$ o atrator de $T(t)$ restrito a $\mathcal{K}_{d}^{+}(N)$, temos que $\mathfrak{A}_{d}(N)$ atrai $\mathfrak{A}_{d}\left(N_{1}\right)$. Portanto, usando novamente a invariância estrita de $\mathfrak{A}_{d}\left(N_{1}\right)$, concluímos que $\mathfrak{A}_{d}\left(N_{1}\right) \subseteq \mathfrak{A}_{d}(N)$ para todo $N_{1} \geqslant N$.

Assim, $\mathfrak{A}_{d}\left(N_{1}\right)=\mathfrak{A}_{d}(N)$, para todo $N_{1} \geqslant N$.

Observemos que

$$
\Theta_{l o c, d}^{+} \stackrel{c}{\hookrightarrow} C\left(\mathbb{R}_{+} ; H^{1-\delta}(\Omega)\right) \cap C^{1}\left(\mathbb{R}_{+} ; H^{-\delta}(\Omega)\right)
$$

para $\delta \in(0,1]$. De fato, basta aplicarmos o Teorema de Compacidade de Lions 1.1 .12 com $E_{0}=L^{2}(\Omega) \times H^{-r}(\Omega), E=H^{1-\delta}(\Omega) \times H^{-\delta}(\Omega), E_{1}=H^{1}(\Omega) \times L^{2}(\Omega)$ e $p_{0}=2$. Temos que $E_{1} \stackrel{c}{\hookrightarrow} E \hookrightarrow E_{0}$. Podemos então enunciar o próximo resultado

Corolário 4.1.15. Se $B \subset \mathcal{K}_{d}^{+}(N)$ (limitado em $\mathcal{F}_{b, d}^{+}$), então

$$
\operatorname{dist}_{C\left([0, T] ; H^{1-\delta}(\Omega)\right) \cap C^{1}\left([0, T] ; H^{-\delta}(\Omega)\right)}\left(\Pi_{[0, T]} T(t) B, \Pi_{[0, T]} \mathcal{K}_{d}\right) \rightarrow 0,
$$

quando $t \rightarrow+\infty$.

Demonstração: É consequência da inclusão (4.52) e da igualdade (4.51).

\subsubsection{Atratores globais para equações hiperbólicas}

Uma vez que construímos o atrator de trajetórias $\mathfrak{A}_{d}$ da equação (4.10), podemos também definir o atrator global para essa equação usando a discussão da Seção 2.2.1.

Seja $u$ uma trajetória da equação (4.10), isto é, $u \in \mathcal{K}_{d}^{+}(N)$ para $N>0$. É conveniente considerarmos o par de funções $\left(u(\cdot), u_{t}(\cdot)\right)$ definidas para $t \geqslant 0$. Das Proposições 4.1.2 e 4.1.10 decorrem que $u \in C_{w}\left(\mathbb{R}_{+} ; H_{d}^{1}(\Omega)\right), u \in C_{w}\left(\mathbb{R}_{+} ; L^{p}(\Omega)\right)$ e $u_{t} \in C_{w}\left(\mathbb{R}_{+} ; L^{2}(\Omega)\right)$. Além disso, sabemos que $u \in L^{\infty}\left(\mathbb{R} ; H_{d}^{1}(\Omega)\right) \cap L^{\infty}\left(\mathbb{R} ; L^{p}(\Omega)\right)$ e $u_{t} \in L^{\infty}\left(\mathbb{R} ; L^{2}(\Omega)\right)$. Portanto, os valores de $\left(u(t), u_{t}(t)\right) \in\left[H_{d}^{1}(\Omega) \cap L^{p}(\Omega)\right] \times L^{2}(\Omega)$ estão bem definidos para $t \geqslant 0$.

Para todo subconjunto $B \subset \mathcal{K}_{d}^{+}(N)$ e para o atrator de trajetórias $\mathfrak{A}_{d}$ da equação (4.10) consideramos as seções

$$
B(t) \doteq\left\{\left(u(t), u_{t}(t)\right): u \in B\right\} \subset\left[H_{d}^{1}(\Omega) \cap L^{p}(\Omega)\right] \times L^{2}(\Omega)
$$

e

$$
\left.\mathfrak{A}_{d}(t) \doteq\left\{\left(u(t), u_{t}(t)\right): u \in \mathfrak{A}_{d}\right\} \subset\left[H_{d}^{1}(\Omega) \cap L^{p}(\Omega)\right] \times L^{2}(\Omega)\right),
$$

para $t \geqslant 0$. 
Definição 4.1.16. O conjunto

$$
\mathcal{A} \doteq \mathfrak{A}(0) \subset\left[H_{d}^{1}(\Omega) \cap L^{p}(\Omega)\right] \times L^{2}(\Omega)
$$

é denominado atrator global da equação (4.10).

Proposição 4.1.17. Se $\mathcal{A}$ é atrator global da equação (4.10), então

(i) $\mathcal{A}$ é limitado, fechado em $\left[H_{d}^{1}(\Omega) \cap L^{p}(\Omega)\right] \times L^{2}(\Omega)$ e compacto em $H^{1-\delta}(\Omega) \times H^{-\delta}(\Omega)$ para $\delta>0$

(ii) $\mathcal{A}$ atrai a seção $B(t)$ de todo subconjunto $B \subset \mathcal{K}_{d}^{+}(N)$, isto é,

$$
\operatorname{dist}_{H^{1-\delta} \times H^{-\delta}}(B(t), \mathcal{A}) \rightarrow 0
$$

quando $t \rightarrow+\infty$;

(iii) $\mathcal{A}$ é o conjunto minimal satisfazendo $(i)$ e $($ ii $)$.

Demonstração: Como o conjunto $\Pi_{[0, T]} \mathfrak{A}_{d}$ é compacto em relação a topologia $\Pi_{[0, T]} \Theta_{l o c, d}^{+}$e de (4.52) temos $\Pi_{[0, T]} \Theta_{l o c, d}^{+} \stackrel{c}{\hookrightarrow} C\left([0, T] ; H^{1-\delta}(\Omega)\right) \cap C^{1}\left([0, T] ; H^{-\delta}(\Omega)\right)$ para $\delta \in(0,1]$, resulta que $\Pi_{[0,0]} \mathfrak{A}_{d}=\mathcal{A}$ é um subconjunto compacto de $H^{1-\delta}(\Omega) \times H^{-\delta}(\Omega)$ e é um subconjunto limitado de $\left[H_{d}^{1}(\Omega) \cap L^{p}(\Omega)\right] \times L^{2}(\Omega)$. Isso demonstra o item $(i)$.

Para demonstrar o item (ii) basta tomarmos $T=0$ em (4.53) o que implica que

$$
\operatorname{dist}_{C\left([0,0] ; H^{1-\delta}(\Omega)\right) \cap C^{1}\left([0,0] ; H^{-\delta}(\Omega)\right)}\left(\Pi_{[0,0]} T(t) B, \Pi_{[0,0]} \mathfrak{A}_{d}\right)=\operatorname{dist}_{H^{1-\delta}(\Omega) \times H^{-\delta}(\Omega)}(B(t), \mathcal{A}) \rightarrow 0
$$

quando $t \rightarrow+\infty$.

Finalmente, suponhamos que exista um conjunto $\mathcal{A}^{\prime}$ fechado em $\left[H_{d}^{1}(\Omega) \cap L^{p}(\Omega)\right] \times L^{2}(\Omega)$ e tal que $\operatorname{dist}_{H^{1-\delta}(\Omega) \times H^{-\delta}(\Omega)}(B(t), \mathcal{A}) \rightarrow 0$ quando $t \rightarrow+\infty$ para todo subconjunto $B \subset \mathcal{K}_{d}^{+}(N)$. Consideremos $B=\mathfrak{A}_{d}$. É claro que $\mathfrak{A}_{d} \subset \mathcal{K}_{d}^{+}(N)$, logo $\operatorname{dist}_{H^{1-\delta}(\Omega) \times H^{-\delta}(\Omega)}\left(\mathfrak{A}_{d}(t), \mathcal{A}^{\prime}\right) \rightarrow 0$ quando $t \rightarrow+\infty$. Entretanto, $\mathfrak{A}_{d}(t)$ não depende de $t$, pois $\mathfrak{A}_{d}$ é estritamente invariante em relação ao semigrupo translação $\{T(t)\}$, ou seja, $T(t) \mathfrak{A}_{d}=\mathfrak{A}_{d}$ para $t \geqslant 0$. Portanto, $\operatorname{dist}_{H^{1-\delta}(\Omega) \times H^{-\delta}(\Omega)}\left(\mathfrak{A}_{d}(0), \mathcal{A}^{\prime}\right)=\operatorname{dist}_{H^{1-\delta}(\Omega) \times H^{-\delta}(\Omega)}\left(\mathcal{A}, \mathcal{A}^{\prime}\right)=0$. Como $\mathcal{A}^{\prime}$ é fechado em $\left[H_{d}^{1}(\Omega) \cap L^{p}(\Omega)\right] \times L^{2}(\Omega)$ decorre que $\mathcal{A} \subseteq \mathcal{A}^{\prime}$. Concluímos que $\mathcal{A}$ está contido em todo conjunto satisfazendo os itens $(i)$ e $(i i)$, isto é, $\mathcal{A}$ é minimal. 


\subsection{Semicontinuidade superior de uma família de atra- tores de trajetórias}

Nessa seção vamos estudar o comportamento assintótico dos atratores de trajetórias dos problemas (4.7) e (4.8) quando $\epsilon \rightarrow 0^{+}$. Se $\left\{\mathfrak{A}_{\epsilon, 1}: \epsilon \in\left[0, \epsilon_{0}\right]\right\}$ e $\left\{\mathfrak{A}_{\epsilon, 2}: \epsilon \in\left[0, \epsilon_{0}\right]\right\}$ são as famílias de atratores de trajetórias de (4.7) e (4.8), respectivamente, então os resultados principais dessa seção demonstram que essas famílias são semicontínuas superiormente em $\epsilon=0$. Para isso é fundamental que as soluções satisfaçam estimativas uniformes em relação a $\epsilon$.

A principal referência para essa seção é o livro de Chepyzhov, V. V. and Vishik, M. I. [9], onde os autores consideraram apenas problemas com condição de Dirichlet.

\subsubsection{Primeiro problema}

Vamos começar com o seguinte problema

$$
\left\{\begin{array}{l}
\frac{\partial^{2} u}{\partial t^{2}}+2 \gamma \frac{\partial u}{\partial t}=d \Delta u-f(u)+\epsilon|u|^{\alpha-1} u \quad(t, x) \in(0, \infty) \times \Omega \\
\frac{\partial u}{\partial \nu}=0 \quad(t, x) \in(0, \infty) \times \Gamma,
\end{array}\right.
$$

onde $\Omega \subset \mathbb{R}^{n}(n \geqslant 3)$ é um subconjunto aberto, limitado, conexo, com fronteira suave $\Gamma$ e para cada ponto $x \in \Gamma, \nu=\nu(x)$ indica o vetor normal exterior unitário a $\Gamma$ no ponto $x$. Além disso, $\gamma>0, d>0$ está fixado, $\alpha \in(0,1), \epsilon \in\left[0, \epsilon_{0}\right], \epsilon_{0}<1$ e $f: \mathbb{R} \rightarrow \mathbb{R}$ é uma função de classe $C^{1}$ satisfazendo (4.11)-(4.13).

Sejam $u \in H_{d}^{1}(\Omega) \cap L^{p}(\Omega), v \in L^{2}(\Omega)$ e consideremos $0<\delta<\min \left\{2 \gamma, 2 \tilde{k_{1}}\right\}$, onde

$\tilde{k_{1}}=\frac{1}{2} \min \left\{1, \gamma_{1}\left(1-\epsilon_{0}\right)\right\}$. Definamos os seguintes funcionais $I_{\epsilon}:\left(H_{d}^{1}(\Omega) \cap L^{p}(\Omega)\right) \times L^{2}(\Omega) \rightarrow \mathbb{R}$ por

$$
I_{\epsilon}(u, v)=\frac{1}{2} \int_{\Omega}\left[|v|^{2}+d|\nabla u|^{2}+2 F(u)-\frac{2 \epsilon}{\alpha+1}|u|^{\alpha+1}\right] d x ;
$$

e $J_{\delta, \epsilon}:\left(H_{d}^{1}(\Omega) \cap L^{p}(\Omega)\right) \times L^{2}(\Omega) \rightarrow \mathbb{R}$ por

$$
J_{\delta, \epsilon}(u, v)=\int_{\Omega}\left[\frac{|v|^{2}}{2}+\frac{d|\nabla u|^{2}}{2}+F(u)-\frac{\epsilon}{\alpha+1}|u|^{\alpha+1}+\delta u \cdot v+\delta \gamma|u|^{2}\right] d x .
$$

Assim como fizemos na demonstração da Proposição 4.1.3, usando a hipótese (4.12), a Desigualdade de Young (1.3) e o fato de $\epsilon \leqslant \epsilon_{0}$, obtemos constantes positivas $k_{1}, k_{2}, k_{3}$ e $k_{4}$ tais que

$$
I_{\epsilon}(u, v) \geqslant k_{1}\left[\|u\|_{H_{d}^{1}(\Omega)}^{2}+\|v\|_{L^{2}(\Omega)}^{2}+\|u\|_{L^{p}(\Omega)}^{p}\right]-k_{2}
$$


onde

$$
k_{1}=\frac{1}{2} \min \left\{1, \gamma_{1}\left(1-\epsilon_{0}\right)\right\}
$$

e

$$
k_{2}=c_{1}|\Omega|+\frac{\epsilon_{0}}{\alpha+1}\left(\frac{2}{\gamma_{1} p}\right)^{\frac{\alpha+1}{p-(\alpha+1)}}|\Omega|\left(\frac{p-(\alpha+1)}{p}\right)+\frac{1}{2}\left(\frac{2}{\gamma_{1} p}\right)^{\frac{2}{p-2}}\left(\frac{p-2}{p}\right)|\Omega|
$$

Se $L$ é uma constante positiva tal que $|F(u)| \leqslant L\left(|u|^{p}+1\right)$, então

$$
I_{\epsilon}(u, v) \leqslant k_{3}\left[\|u\|_{H_{d}^{1}(\Omega)}^{2}+\|v\|_{L^{2}(\Omega)}^{2}+\|u\|_{L^{p}(\Omega)}^{p}\right]+k_{4}
$$

onde

$$
k_{3}=\frac{1}{2} \max \left\{1, L\left(2+\epsilon_{0}\right)\right\}
$$

e

$$
k_{4}=L|\Omega|+\frac{\epsilon_{0}}{\alpha+1}\left(\frac{1}{L p}\right)^{\frac{\alpha+1}{p-(\alpha+1)}}|\Omega|\left(\frac{p-(\alpha+1)}{p}\right)
$$

Temos também

$$
\begin{aligned}
J_{\delta, \epsilon}(u, v) & =I_{\epsilon}(u, v)+\delta \int_{\Omega}\left(u \cdot v+\gamma|u|^{2}\right) d x \\
& \geqslant\left(k_{1}-\frac{\delta}{2}\right)\left[\|u\|_{H_{d}^{1}(\Omega)}^{2}+\|v\|_{L^{2}(\Omega)}^{2}+\|u\|_{L^{p}(\Omega)}^{p}\right]-k_{2}
\end{aligned}
$$

e

$$
J_{\delta, \epsilon}(u, v) \leqslant\left(k_{3}+\frac{\delta}{2}+\delta \gamma\right)\left[\|u\|_{H_{d}^{1}(\Omega)}^{2}+\mid v\left\|_{L^{2}(\Omega)}^{2}+\right\| u \|_{L^{p}(\Omega)}^{p}\right]+k_{4}
$$

Analogamente para $\epsilon=0$, existem constantes positivas $\tilde{k_{1}}, \tilde{k_{2}}, \tilde{k_{3}}$ e $\tilde{k_{4}}$ tais que

$$
I_{0}(u, v) \geqslant \tilde{k_{1}}\left[\|u\|_{H_{d}^{1}(\Omega)}^{2}+\|v\|_{L^{2}(\Omega)}^{2}+\|u\|_{L^{p}(\Omega)}^{p}\right]-\tilde{k_{2}}
$$

onde

$$
\tilde{k_{1}}=\frac{1}{2} \min \left\{1, \gamma_{1}\right\}
$$


e

$$
\begin{gathered}
\tilde{k_{2}}=c_{1}|\Omega|+\frac{1}{2}\left(\frac{2}{\gamma_{1} p}\right)^{\frac{2}{p-2}}|\Omega| \frac{p-2}{p} ; \\
I_{0}(u, v) \leqslant \tilde{k_{3}}\left[\|u\|_{H_{d}^{1}(\Omega)}^{2}+\|v\|_{L^{2}(\Omega)}^{2}+\|u\|_{L^{p}(\Omega)}^{p}\right]+\tilde{k_{4}},
\end{gathered}
$$

onde

$$
\tilde{k_{3}}=\frac{1}{2} \max \{1,2 L\}
$$

e

$$
\tilde{k}_{4}=\tilde{L}|\Omega| .
$$

Além disso,

$$
\begin{aligned}
J_{\delta, 0}(u, v) & =I_{0}(u, v)+\delta \int_{\Omega}\left(u \cdot v+\gamma|u|^{2}\right) d x \\
& \geqslant\left(\tilde{k_{1}}-\frac{\delta}{2}\right)\left[\|u\|_{H_{d}^{1}(\Omega)}^{2}+\|v\|_{L^{2}(\Omega)}^{2}+\|u\|_{L^{p}(\Omega)}^{p}\right]-\tilde{k_{2}}
\end{aligned}
$$

e

$$
J_{\delta, 0}(u, v) \leqslant\left(\tilde{k_{3}}+\frac{\delta}{2}+\delta \gamma\right)\left[\|u\|_{H_{d}^{1}(\Omega)}^{2}+\|v\|_{L^{2}(\Omega)}^{2}+\|u\|_{L^{p}(\Omega)}^{p}\right]+\tilde{k_{4}}
$$

Vamos identificar abaixo os conjuntos $\mathcal{F}_{l o c, \epsilon}^{+}, \mathcal{F}_{b, \epsilon}^{+}, \mathcal{F}_{l o c, 0}^{+}, \mathcal{F}_{b, 0}^{+}$e as topologias $\Theta_{l o c, \epsilon}^{+}$e $\Theta_{l o c, 0}^{+}:$

$$
\begin{gathered}
\mathcal{F}_{l o c, \epsilon}^{+}=\left\{v: v \in L_{l o c}^{\infty}\left(\mathbb{R}_{+} ; L^{p}(\Omega)\right) \cap L_{l o c}^{\infty}\left(\mathbb{R}_{+} ; H_{d}^{1}(\Omega)\right), \partial_{t} v \in L_{l o c}^{\infty}\left(\mathbb{R}_{+} ; L^{2}(\Omega)\right) \mathrm{e}\right. \\
\left.\partial_{t}^{2} v \in L_{l o c}^{\infty}\left(\mathbb{R}_{+} ; H^{-r}(\Omega)\right)\right\}=\mathcal{F}_{l o c, 0}^{+} ; \\
\mathcal{F}_{b, \epsilon}^{+}=\left\{v: v \in \mathcal{F}_{l o c, \epsilon}^{+} \operatorname{com}\|v\|_{\mathcal{F}_{b, \epsilon}^{+}}<+\infty\right\}=\mathcal{F}_{b, 0}^{+},
\end{gathered}
$$

onde

$$
\begin{aligned}
\|v\|_{\mathcal{F}_{b, \epsilon}^{+}}= & \|v\|_{\mathcal{F}_{b, 0}^{+}}=\|v\|_{L^{\infty}\left(\mathbb{R}_{+} ; L^{p}(\Omega)\right)}+\|v\|_{L^{\infty}\left(\mathbb{R}_{+} ; H_{d}^{1}(\Omega)\right)}+\left\|\partial_{t} v\right\|_{L^{\infty}\left(\mathbb{R}_{+} ; L^{2}(\Omega)\right)}+ \\
& +\left\|\partial_{t}^{2} v\right\|_{L^{\infty}\left(\mathbb{R}_{+} ; H^{-r}(\Omega)\right)}
\end{aligned}
$$


E munimos $\mathcal{F}_{l o c, \epsilon}^{+}$da topologia $\Theta_{l o c, \epsilon}^{+}$definida da seguinte forma: $v_{m} \rightarrow v$ em $\Theta_{l o c, \epsilon}^{+}$quando $\Pi_{[0, T]} v_{m} \rightarrow \Pi_{[0, T]} v$ em $\Theta_{T, \epsilon}$ para todo $T>0$. Essa definição é válida para $\epsilon \in\left[0, \epsilon_{0}\right]$.

Pelo Teorema 4.1 .8 existe pelo menos uma solução fraca global $u_{\epsilon}$ para a equação (4.54) quando $\epsilon \in\left[0, \epsilon_{0}\right]$. O conjunto de trajetórias $\mathcal{K}_{\epsilon}^{+}(N)$ da equação (4.54) quando $\epsilon>0$ é o conjunto das soluções fracas globais $u_{\epsilon}$ da equação (4.54) que satisfazem

$$
\begin{aligned}
& \frac{d}{d t} J_{\delta, \epsilon}\left(u_{\epsilon}, \partial_{t} u_{\epsilon}\right) \leqslant-\min \left\{2 \gamma-\delta, \delta, \frac{\delta \gamma_{1} \gamma_{2}\left(1-\epsilon_{0}\right)}{2}\right\}\left[\left\|u_{\epsilon}\right\|_{H_{d}^{1}(\Omega)}^{2}+\left\|\partial_{t} u_{\epsilon}\right\|_{L^{2}(\Omega)}^{2}+\right. \\
& \left.+\left\|u_{\epsilon}\right\|_{L^{p}(\Omega)}^{p}\right]+\left[c_{2}+c_{1} \gamma_{2}\right]|\Omega| \delta+\delta\left(\frac{4}{\gamma_{1} \gamma_{2} p}\right)^{\frac{2}{p-2}}|\Omega|\left(\frac{p-2}{p}\right)+ \\
& +\epsilon_{0} \delta\left(\frac{2(\alpha+1)}{\gamma_{1} \gamma_{2} p}\right)^{\frac{\alpha+1}{p-(\alpha+1)}}\left(\frac{p-(\alpha+1)}{p}\right)|\Omega| .
\end{aligned}
$$

De $(4.57)$ e $(4.55)$, obtemos

$$
\frac{d}{d t} J_{\delta, \epsilon}\left(u_{\epsilon}, \partial_{t} u_{\epsilon}\right) \leqslant-k_{5} J_{\delta, \epsilon}\left(u_{\epsilon}, \partial_{t} u_{\epsilon}\right)+k_{6},
$$

onde

$$
k_{5}=\frac{\min \left\{2 \gamma-\delta, \delta, \frac{\delta \gamma_{1} \gamma_{2}\left(1-\epsilon_{0}\right)}{2}\right\}}{k_{3}+\frac{\delta}{2}+\delta \gamma}
$$

e

$$
\begin{aligned}
k_{6}= & k_{4} k_{5}+\left[c_{2}+c_{1} \gamma_{2}\right]|\Omega| \delta+\delta\left(\frac{4}{\gamma_{1} \gamma_{2} p}\right)^{\frac{2}{p-2}}|\Omega|\left(\frac{p-2}{p}\right)+ \\
& +\epsilon_{0} \delta\left(\frac{2(\alpha+1)}{\gamma_{1} \gamma_{2} p}\right)^{\frac{\alpha+1}{p-(\alpha+1)}}\left(\frac{p-(\alpha+1)}{p}\right)|\Omega| .
\end{aligned}
$$

E de (4.58), concluímos que

$$
z_{\epsilon}(t)=J_{\delta, \epsilon}\left(u_{\epsilon}, \partial_{t} u_{\epsilon}\right) \leqslant z_{\epsilon}(0) e^{-k_{5} t}+\frac{k_{6}}{k_{5}} \leqslant N e^{-k_{5} t}+\frac{k_{6}}{k_{5}}
$$

para todo $t \geqslant 0$.

Agora, para $\epsilon=0$ o conjunto de trajetórias $\mathcal{K}_{0}^{+}(N)$ da equação (4.54) é o conjunto das soluções fracas globais $u$ que verificam a desigualdade

$$
\frac{d}{d t} J_{\delta, 0}\left(u, \partial_{t} u\right) \leqslant-\min \left\{2 \gamma-\delta, \delta, \frac{\delta \gamma_{1} \gamma_{2}}{2}\right\}\left[\|u\|_{H_{d}^{1}(\Omega)}^{2}+\left\|\partial_{t} u\right\|_{L^{2}(\Omega)}^{2}+\right.
$$




$$
\left.+\|u\|_{L^{p}(\Omega)}^{p}\right]+\left[c_{2}+c_{1} \gamma_{2}\right]|\Omega| \delta+\delta\left(\frac{4}{\gamma_{1} \gamma_{2} p}\right)^{\frac{2}{p-2}}|\Omega|\left(\frac{p-2}{p}\right) .
$$

De (4.59) e (4.56), obtemos

$$
\frac{d}{d t} J_{\delta, 0}\left(u, \partial_{t} u\right) \leqslant-\tilde{k_{5}} J_{\delta, 0}\left(u, \partial_{t} u\right)+\tilde{k_{6}}
$$

onde

$$
\tilde{k_{5}}=\frac{\min \left\{2 \gamma-\delta, \delta, \frac{\delta \gamma_{1} \gamma_{2}}{2}\right\}}{\tilde{k_{3}}+\frac{\delta}{2}+\delta \gamma}
$$

e

$$
\tilde{k_{6}}=\tilde{k_{4}} \tilde{k_{5}}+\left[c_{2}+c_{1} \gamma_{2}\right]|\Omega| \delta+\delta\left(\frac{4}{\gamma_{1} \gamma_{2} p}\right)^{\frac{2}{p-2}}|\Omega|\left(\frac{p-2}{p}\right)
$$

E de (4.60), concluímos que

$$
z(t)=J_{\delta, 0}\left(u, \partial_{t} u\right) \leqslant N e^{-\tilde{k_{5}} t}+\frac{\tilde{k_{6}}}{\tilde{k_{5}}}
$$

para todo $t \geqslant 0$.

De acordo com a Proposição 4.1.10, podemos afirmar que os conjuntos de trajetórias $\mathcal{K}_{\epsilon}^{+}(N)$ e $\mathcal{K}_{0}^{+}(N)$ estão contidos em $\mathcal{F}_{b, \epsilon}^{+}$e $\mathcal{F}_{b, 0}^{+}$, respectivamente. Vamos munir esses conjuntos das topologias $\Theta_{l o c, \epsilon}^{+}$e $\Theta_{l o c, 0}^{+}$, respectivamente e denominá-los espaços de trajetórias.

O semigrupo translação $\left\{T(t): \mathcal{K}_{\epsilon}^{+}(N) \rightarrow \mathcal{K}_{\epsilon}^{+}(N): t \geqslant 0\right\}$ possui os seguintes conjuntos atrativos

$$
P_{\epsilon}=\left\{u \in \mathcal{K}_{\epsilon}^{+}: z_{\epsilon}(t)=J_{\delta, \epsilon}\left(u, \partial_{t} u\right) \leqslant \frac{2 k_{6}}{k_{5}}\right\}
$$

para $\epsilon>0$ e

$$
P_{0}=\left\{u \in \mathcal{K}_{0}^{+}: z(t)=J_{\delta, 0}\left(u, \partial_{t} u\right) \leqslant \frac{2 \tilde{k_{6}}}{\tilde{k_{5}}}\right\}
$$

para $\epsilon=0$.

Em virtude do Teorema 1.3.10, o semigrupo translação $\{T(t): t \geqslant 0\}$ possui um atrator de trajetórias $\mathfrak{A}_{\epsilon}(N) \equiv \mathfrak{A}_{\epsilon}$ para $\epsilon \in\left[0, \epsilon_{0}\right]$ que independe de $N$. 
Indicaremos por $\mathcal{K}_{\epsilon}$ e $\mathcal{K}_{0}$ os núcleos da equação

$$
\frac{\partial^{2} u}{\partial t^{2}}+2 \gamma \frac{\partial u}{\partial t}=d \Delta u-f(u)+\epsilon|u|^{\alpha-1} u \quad(t, x) \in \mathbb{R} \times \Omega
$$

quando $\epsilon>0$ e $\epsilon=0$, respectivamente. Em razão do Teorema 4.1.13, decorre que

$$
\mathfrak{A}_{\epsilon}=\Pi_{+} \mathcal{K}_{\epsilon}
$$

para $\epsilon \in\left[0, \epsilon_{0}\right]$.

Vamos enunciar o resultado principal dessa subseção.

Teorema 4.2.1. Se $\mathfrak{A}_{\epsilon}$ é o atrator de trajetórias da equação (4.54) com $\epsilon \in\left[0, \epsilon_{0}\right]$, então a familia de atratores $\left\{\mathfrak{A}_{\epsilon}: \epsilon \in\left[0, \epsilon_{0}\right]\right\}$ é semicontínua superiormente em $\epsilon=0$.

Demonstração: Suponhamos que a tese do enunciado é falsa. Logo, existem uma vizinhança $\mathcal{O}_{[-T, T], 0}$ de $\mathcal{K}_{0}$ em relação a topologia $\Theta_{[-T, T], 0}$ e sequências $\left\{\epsilon_{m}: \epsilon_{m}>0\right\}, \epsilon_{m} \rightarrow 0^{+}$, $\left\{u_{\epsilon_{m}}^{m}\right\} \subset \mathcal{K}_{\epsilon_{m}}$ tais que

$$
u_{\epsilon_{m}}^{m} \notin \mathcal{O}_{[-T, T], 0}\left(\mathcal{K}_{0}\right)
$$

para $\operatorname{algum} T>0$.

Temos que

$$
\begin{aligned}
& J_{\delta, 0}\left(u_{\epsilon_{m}}^{m}, \partial_{t} u_{\epsilon_{m}}^{m}\right)=J_{\delta, u_{\epsilon_{m}}^{m}}\left(u_{\epsilon_{m}}^{m}, \partial_{t} u_{\epsilon_{m}}^{m}\right)+\frac{\epsilon_{m}}{\alpha+1} \int_{\Omega}\left|u_{\epsilon_{m}}^{m}\right|^{\alpha+1} d x \leqslant \\
& \leqslant z_{\epsilon_{m}}+\frac{\epsilon_{m}}{\alpha+1}\left(\operatorname{ess} \sup \left\|u_{\epsilon_{m}}^{m}\right\|_{L^{\alpha+1}}\right)^{\alpha+1}|\Omega|<+\infty
\end{aligned}
$$

para $\epsilon_{m} \in\left[0, \epsilon_{0}\right]$, pois $L^{2}(\Omega) \hookrightarrow L^{\alpha+1}(\Omega)$.

Portanto, a sequência $\left\{u_{\epsilon_{m}}^{m}\right\}$ é limitada na norma do espaço $\mathcal{F}_{b, 0}$. Assim, existe uma subsequência indicada por $\left\{u_{\epsilon_{m}}^{m}\right\}$ tal que $u_{\epsilon_{m}}^{m} \rightarrow \tilde{u}$ em $\Theta_{l o c, 0}$. Observemos que $\tilde{u}$ também satisfaz (4.63).

Agora, tomando o limite em (4.63) quando $m \rightarrow+\infty$, obtemos

$$
\begin{aligned}
z(t) & =J_{\delta, 0}\left(\tilde{u}, \partial_{t} \tilde{u}\right) \leqslant \liminf _{m \rightarrow \infty} J_{\delta, 0}\left(u_{\epsilon_{m}}^{m}, \partial_{t} u_{\epsilon_{m}}^{m}\right) \\
& \leqslant \lim _{m \rightarrow \infty}\left[z_{\epsilon_{m}}+\frac{\epsilon_{m}}{\alpha+1}\left(\operatorname{ess} \sup \left\|u_{\epsilon_{m}}^{m}\right\|_{L^{\alpha+1}}\right)^{\alpha+1}|\Omega|\right] \leqslant \frac{\tilde{k_{6}}}{\tilde{k_{5}}}
\end{aligned}
$$

Vamos checar que $\tilde{u} \in \mathcal{K}_{0}$. Em razão de (4.64) é suficiente demonstrar que $\tilde{u}$ é uma solução fraca da equação (4.54) $\operatorname{com} \epsilon=0$. 
A função $u_{\epsilon_{m}}^{m}$ obviamente satisfaz a equação

$$
\partial_{t}^{2} u_{\epsilon_{m}}^{m}+2 \gamma \partial_{t} u_{\epsilon_{m}}^{m}=d \Delta u_{\epsilon_{m}}^{m}-f\left(u_{\epsilon_{m}}^{m}\right)+\epsilon_{m}\left|u_{\epsilon_{m}}^{m}\right|^{\alpha-1} u_{\epsilon_{m}}^{m}
$$

no sentido de distribuição do espaço $D^{\prime}\left(\mathbb{R} ; H^{-r}(\Omega)\right)$.

Vamos analisar o termo a seguir. Temos que

$$
\int_{\Omega} \epsilon_{m}\left|u_{\epsilon_{m}}^{m}\right|^{\alpha} d x \leqslant \epsilon_{m}\left(\int_{\Omega}\left|u_{\epsilon_{m}}^{m}\right|^{\alpha q} d x\right)^{\frac{1}{q}}\left(\int_{\Omega} d x\right)^{\frac{1}{p}} \rightarrow 0
$$

quando $m \rightarrow \infty$, $\operatorname{logo} \epsilon_{m}\left|u_{\epsilon_{m}}^{m}\right|^{\alpha-1} u_{\epsilon_{m}}^{m} \rightarrow 0$ em $D^{\prime}\left(\mathbb{R} ; H^{-r}(\Omega)\right)$ quando $m \rightarrow \infty$. Quanto aos outros termos de (4.65) já sabemos que podemos tomar o limite quando $m \rightarrow+\infty$.

Logo, tomando o limite quando $m \rightarrow+\infty$ na equação (4.65) ficamos com a igualdade

$$
\partial_{t}^{2} \tilde{u}+2 \gamma \partial_{t} \tilde{u}=d \Delta \tilde{u}-f(\tilde{u})
$$

no espaço de distribuição $D^{\prime}\left(\mathbb{R} ; H^{-r}(\Omega)\right)$.

Portanto, $\tilde{u}$ pertence ao espaço $\mathcal{K}_{0}$. Por outro lado, a sequência $\left\{u_{\epsilon_{m}}^{m}\right\}$ converge para a função $\tilde{u}$ na topologia $\Theta_{l o c, 0}$ e, em particular, em $\Theta_{[-T, T], 0}$ para $T>0$. Porém, isso contradiz a nossa hipótese (4.62) e assim concluímos a demonstração.

Usando o resultado de imersão $\Theta_{l o c, \epsilon}^{+} \stackrel{c}{\hookrightarrow} C\left(\mathbb{R}_{+} ; H^{1-\delta}(\Omega)\right) \cap C^{1}\left(\mathbb{R}_{+} ; H^{-\delta}(\Omega)\right)$ para $\delta \in(0,1]$ e para $\epsilon \in\left[0, \epsilon_{0}\right]$, obtemos

\section{Corolário 4.2.2.}

$$
\operatorname{dist}_{C\left([0, T] ; H^{1-\delta}(\Omega)\right)}\left(\Pi_{[0, T]} \mathfrak{A}_{\epsilon}, \Pi_{[0, T]} \mathfrak{A}_{0}\right)+\operatorname{dist}_{C^{1}\left([0, T] ; H^{-\delta}(\Omega)\right)}\left(\Pi_{[0, T]} \mathfrak{A}_{\epsilon}, \Pi_{[0, T]} \mathfrak{A}_{0}\right) \rightarrow 0
$$

quando $\epsilon \rightarrow 0^{+}$, para todo $T>0$.

Seja $\mathcal{A}_{\epsilon}$ o atrator global de (4.54) definido na Seção 4.1.1. Fazendo $T=0$ em (4.66), podemos enunciar o próximo resultado.

Corolário 4.2.3. Para $0<\delta \leqslant 1$ decorre que

$$
\operatorname{dist}_{H^{1-\delta}(\Omega) \times H^{-\delta}(\Omega)}\left(\mathcal{A}_{\epsilon}, \mathcal{A}_{0}\right) \rightarrow 0
$$

quando $\epsilon \rightarrow 0^{+}$. 
4 Atrator de trajetórias de uma equação hiperbólica

\subsubsection{Segundo problema}

Vamos agora estudar o caso em que o parâmetro pequeno $\epsilon>0$ aparece multiplicando o termo da derivada de maior ordem da equação hiperbólica, ou seja,

$$
\left\{\begin{array}{l}
\epsilon \frac{\partial^{2} u}{\partial t^{2}}+2 \gamma u_{t}=d \Delta u-f(u)+|u|^{\alpha-1} u \quad(t, x) \in(0, \infty) \times \Omega \\
\frac{\partial u}{\partial \nu}=0 \quad(t, x) \in(0, \infty) \times \Gamma
\end{array}\right.
$$

onde $\Omega \subset \mathbb{R}^{n}(n \geqslant 3)$ é um subconjunto aberto, limitado, conexo, com fronteira suave $\Gamma$ e para cada ponto $x \in \Gamma, \nu=\nu(x)$ indica o vetor normal exterior unitário a $\Gamma$ no ponto $x$. Além disso, $\gamma>0, d>0$ está fixado, $\alpha \in(0,1), \epsilon \in\left[0, \epsilon_{0}\right], \epsilon_{0}<1$ e $f: \mathbb{R} \rightarrow \mathbb{R}$ é uma função de classe $C^{1}$ satisfazendo (4.11)-(4.13).

Observação 4.2.1. No livro de Chepyzhov, V. V. and Vishik, M. I. [9], os autores consideraram o problema hiperbólico

$$
\epsilon \frac{\partial^{2} u}{\partial t^{2}}+\gamma u_{t}=d \Delta u-f(u)+g(x),\left.\quad u\right|_{\Gamma}=0,
$$

onde $f$ satisfaz (4.11)-(4.13) e existe constante $\tilde{C}$ tal que

$$
\left(f\left(v_{1}\right)-f\left(v_{2}\right)\right)\left(v_{1}-v_{2}\right) \geqslant-\tilde{C}\left(v_{1}-v_{2}\right)^{2}, \quad \text { para quaisquer } v_{1}, v_{2} \in \mathbb{R}
$$

e demonstraram a semicontinuidade superior da familia de atratores de trajetórias $\left\{\mathfrak{A}_{\epsilon}: \epsilon \in\right.$ $\left.\left[0, \epsilon_{0}\right]\right\}$ em $\epsilon=0$. Mas observamos que neste caso a equação limite parabólica admite solução única em virtude da hipótese (4.68). Agora, no artigo de Lyapin, A. S. [31], o autor demonstrou o mesmo resultado para um problema mais geral, não autônomo, em que a equação limite parabólica pode ter mais de uma solução.

Definição 4.2.4. Uma função $u=u(t, x), t \in[0, T], x \in \Omega$, é uma solução fraca global de (4.67) para $\epsilon>0$ se $u \in L^{\infty}\left(0, T ; L^{p}(\Omega)\right) \cap L^{\infty}\left(0, T ; H_{d}^{1}(\Omega)\right), u_{t} \in L^{\infty}\left(0, T ; L^{2}(\Omega)\right)$ e u satisfaz a equação (4.67) no sentido de distribuição do espaço $D^{\prime}\left(0, T ; H^{-r}(\Omega)\right.$ ), para todo $T>0$, onde $r=\max \left\{1, n\left(\frac{1}{q}-\frac{1}{2}\right)\right\}$; Enquanto para $\epsilon=0$, uma solução é uma função u tal que $u \in L^{\infty}\left(0, T ; L^{p}(\Omega)\right) \cap L^{\infty}\left(0, T ; H_{d}^{1}(\Omega)\right)$ e $u_{t} \in L_{b}^{2}\left(0, T ; L^{2}(\Omega)\right)$, para todo $T>0$.

Proposição 4.2.5. Se $u_{0} \in H_{d}^{1}(\Omega) \cap L^{p}(\Omega)$, então existe pelo menos uma solução fraca $u \in L^{\infty}\left(0, T ; L^{p}(\Omega)\right) \cap L^{\infty}\left(0, T ; H_{d}^{1}(\Omega)\right), \partial_{t} u \in L_{b}^{2}\left(0, T ; L^{2}(\Omega)\right)$ da equação (4.67) com $\epsilon=0$ tal que $u(0)=u_{0}$.

Demonstração: A demonstração é análoga a que fizemos para o Teorema 3.1.2.

Sejam $u \in H_{d}^{1}(\Omega) \cap L^{p}(\Omega), v \in L^{2}(\Omega)$ e considere $0<\delta<\min \left\{1, \frac{2 \gamma}{\epsilon_{0}}, \frac{2 k_{1}}{\epsilon_{0}}\right\}$, onde 
$k_{1}=\frac{1}{2} \min \left\{1, \tilde{\gamma}_{1}\right\}$. Definamos os seguintes funcionais $I_{\epsilon}:\left(H_{d}^{1}(\Omega) \cap L^{p}(\Omega)\right) \times L^{2}(\Omega) \rightarrow \mathbb{R}$ por

$$
I_{\epsilon}(u, v)=\frac{1}{2} \int_{\Omega}\left[\epsilon|v|^{2}+d|\nabla u|^{2}+2 \tilde{F}(u)\right] d x
$$

e $J_{\delta, \epsilon}:\left(H_{d}^{1}(\Omega) \cap L^{p}(\Omega)\right) \times L^{2}(\Omega) \rightarrow \mathbb{R}$ por

$$
J_{\delta, \epsilon}(u, v)=\int_{\Omega}\left[\frac{\epsilon|v|^{2}}{2}+\frac{d|\nabla u|^{2}}{2}+\tilde{F}(u)+\epsilon \delta u \cdot v+\delta \gamma|u|^{2}\right] d x .
$$

Usando a hipótese (4.15), a Desigualdade de Young (1.3), $\epsilon \leqslant \epsilon_{0}$ e considerando uma constante positiva $\tilde{L}$ tal que $|\tilde{F}(u)| \leqslant \tilde{L}\left(1+|u|^{p}\right)$, podemos determinar constantes positivas $k_{1}, k_{2}, k_{3}$ e $k_{4}$ satisfazendo

$$
I_{\epsilon}(u, v) \geqslant k_{1}\left[\|u\|_{H_{d}^{1}(\Omega)}^{2}+\|u\|_{L^{p}(\Omega)}^{p}\right]+\frac{\epsilon\|v\|^{2}}{2}-k_{2}
$$

onde

$$
k_{1}=\frac{1}{2} \min \left\{1, \tilde{\gamma}_{1}\right\}
$$

e

$$
\begin{gathered}
k_{2}=\tilde{c_{1}}|\Omega|+\frac{1}{2}\left(\frac{2}{\tilde{\gamma}_{1} p}\right)^{\frac{2}{p-2}}\left(\frac{p-2}{p}\right)|\Omega| ; \\
I_{\epsilon}(u, v) \leqslant k_{3}\left[\|u\|_{H_{d}^{1}(\Omega)}^{2}+\|v\|_{L^{2}(\Omega)}^{2}+\|u\|_{L^{p}(\Omega)}^{p}\right]+k_{4},
\end{gathered}
$$

onde

$$
k_{3}=\frac{1}{2} \max \left\{1,2 L, \epsilon_{0}\right\}
$$

e

$$
k_{4}=L|\Omega| .
$$

Além disso,

$$
\begin{aligned}
J_{\delta, \epsilon}(u, v) & =I_{\epsilon}(u, v)+\delta \int_{\Omega}\left(\epsilon u \cdot v+\gamma|u|^{2}\right) d x \\
& \geqslant\left(k_{1}-\frac{\delta \epsilon_{0}}{2}\right)\left[\|u\|_{H_{d}^{1}(\Omega)}^{2}+\|u\|_{L^{p}(\Omega)}^{p}\right]+\epsilon(1-\delta) \frac{\|v\|^{2}}{2}-k_{2}
\end{aligned}
$$


$\mathrm{e}$

$$
\begin{aligned}
J_{\delta, \epsilon}(u, v) & =I_{\epsilon}(u, v)+\delta \int_{\Omega}\left(\epsilon u \cdot v+\gamma|u|^{2}\right) d x \\
& \leqslant\left(k_{3}+\frac{\delta \epsilon_{0}}{2}+\delta \gamma\right)\left[\|u\|_{H_{d}^{1}(\Omega)}^{2}+\|v\|_{L^{2}(\Omega)}^{2}+\|u\|_{L^{p}(\Omega)}^{p}\right]+k_{4} .
\end{aligned}
$$

Pelo Teorema 4.1.8, existe pelo menos uma solução fraca global $u_{\epsilon}$ para a equação (4.67) quando $\epsilon \in\left(0, \epsilon_{0}\right]$.

Proposição 4.2.6. Se $u_{\epsilon}$ é uma solução fraca de (4.67) para $\epsilon>0$ e $z_{\epsilon}(t)=J_{\delta, \epsilon}\left(u_{\epsilon}, \partial u_{\epsilon}\right)$, então existem constantes positivas $\mu_{\delta}$ e $\rho_{\delta}$ tais que

$$
z_{\epsilon}(t) \leqslant e^{-\mu_{\delta} t} z_{\epsilon}(0)+\frac{\rho_{\delta}}{\mu_{\delta}}
$$

para $t \geqslant 0$.

Demonstração: Procedendo como na demonstração do Teorema 4.1.6 e usando a hipótese (4.16), obtemos

$$
\begin{aligned}
& \frac{d}{d t} J_{\delta, \epsilon}\left(u_{\epsilon}, \partial_{t} u_{\epsilon}\right) \leqslant-\min \left\{2 \gamma-\epsilon_{0} \delta, \delta, \frac{\tilde{\gamma_{2}} \delta}{2}\right\}\left[\left\|u_{\epsilon}\right\|_{H_{d}^{1}(\Omega)}^{2}+\left\|\partial_{t} u_{\epsilon}\right\|_{L^{2}(\Omega)}^{2}+\left\|u_{\epsilon}\right\|_{L^{p}(\Omega)}^{p}\right]+ \\
& +\tilde{c_{2}}|\Omega| \delta+\delta\left(\frac{4}{\tilde{\gamma_{2} p}}\right)^{\frac{2}{p-2}}|\Omega|\left(\frac{p-2}{p}\right) .
\end{aligned}
$$

De (4.71) e de (4.69), temos que

$$
\frac{d}{d t} J_{\delta, \epsilon}\left(u_{\epsilon}, \partial_{t} u_{\epsilon}\right) \leqslant-k_{5} J_{\delta, \epsilon}\left(u_{\epsilon}, \partial_{t} u_{\epsilon}\right)+k_{6},
$$

onde

$$
k_{5}=\frac{\min \left\{2 \gamma-\epsilon_{0} \delta, \delta, \frac{\tilde{\gamma_{2}} \delta}{2}\right\}}{k_{3}+\frac{\delta \epsilon_{0}}{2}+\delta \gamma}
$$

e

$$
k_{6}=k_{4} k_{5}+\tilde{c_{2}}|\Omega| \delta+\delta\left(\frac{4}{\tilde{\gamma_{2}} p}\right)^{\frac{2}{p-2}}|\Omega|\left(\frac{p-2}{p}\right) .
$$

De (4.72), decorre que

$$
z_{\epsilon}(t)=J_{\delta, \epsilon}\left(u_{\epsilon}, \partial_{t} u_{\epsilon}\right) \leqslant z_{\epsilon}(0) e^{-k_{5} t}+\frac{k_{6}}{k_{5}},
$$


para todo $t \geqslant 0$. Portanto, $\mu_{\delta}=k_{5}$ e $\rho_{\delta}=k_{6}$.

Vamos obter mais uma estimativa a priori para $\partial_{t} u_{\epsilon}$.

Fazendo $\delta=0$ em $z_{\epsilon}(t)$, obtemos

$\frac{d}{d t}\left[\frac{\epsilon}{2}\left\|\partial_{t} u_{\epsilon}\right\|_{L^{2}(\Omega)}^{2}+\frac{d}{2}\left\|\nabla u_{\epsilon}\right\|_{L^{2}(\Omega)}^{2}+\int_{\Omega} \tilde{F}\left(u_{\epsilon}\right) d x\right]+2 \gamma \int_{\Omega}\left|\partial_{t} u_{\epsilon}\right|^{2} d x \leqslant 0$.

Integrando (4.73) em $[t, t+1]$, resulta que

$2 \gamma \int_{t}^{t+1}\left\|\partial_{t} u_{\epsilon}\right\|_{L^{2}(\Omega)}^{2} d t \leqslant \frac{d}{2}\left\|\nabla u_{\epsilon}(t)\right\|_{L^{2}(\Omega)}^{2}+\frac{\epsilon}{2}\left\|\partial_{t} u_{\epsilon}(t)\right\|_{L^{2}(\Omega)}^{2}+\int_{\Omega} \tilde{F}\left(u_{\epsilon}(t)\right) d x+\tilde{c_{1}}|\Omega|$

Observemos que em (4.74) negligenciamos os termos positivos $\frac{d}{2}\left\|\nabla u_{\epsilon}(t+1)\right\|_{L^{2}(\Omega)}^{2}$, $\epsilon\left\|\partial_{t} u_{\epsilon}(t+1)\right\|_{L^{2}(\Omega)}^{2}, \int_{\Omega}\left[\tilde{F}\left(u_{\epsilon}(t+1)\right)+\tilde{c_{1}}\right] d x$.

De (4.74), concluímos que

$$
2 \gamma \int_{t}^{t+1}\left\|\partial_{t} u_{\epsilon}\right\|_{L^{2}(\Omega)}^{2} d t \leqslant z_{\epsilon}(t)+\tilde{c_{1}}|\Omega| \leqslant z_{\epsilon}(0) e^{-\mu_{\delta} t}+\rho_{\delta}+\tilde{c_{1}}|\Omega| .
$$

Definição 4.2.7. Para $0<\epsilon \leqslant \epsilon_{0}$, o espaço de trajetórias $\mathcal{K}_{\epsilon}^{+}(N)$ da equação (4.67) é o conjunto de todas as soluções fracas $u_{\epsilon}$ satisfazendo as desigualdades

$$
z_{\epsilon}(t) \leqslant z_{\epsilon}(0) e^{-\mu_{\delta} t}+\frac{\rho_{\delta}}{\mu_{\delta}}
$$

$e$

$$
2 \gamma \int_{t}^{t+1}\left\|\partial_{t} u_{\epsilon}\right\|_{L^{2}(\Omega)}^{2} d t \leqslant z_{\epsilon}(0) e^{-\mu_{\delta} t}+\rho_{\delta}+\tilde{c_{1}}|\Omega|
$$

para $t \geqslant 0$.

Além disso, para $\epsilon=0$, o espaço de trajetórias $\mathcal{K}_{0}^{+}$da equação (4.67) é o conjunto de todas as soluções fracas $u$.

Observação 4.2.2. As funções de $\mathcal{K}_{0}^{+}$satisfazem (4.75) e (4.76).

Observação 4.2.3. Das Proposições 4.1.8 e 4.2.5 decorre que o conjunto de trajetórias $\mathcal{K}_{\epsilon}^{+}(N)$ é não vazio para $\epsilon \in\left[0, \epsilon_{0}\right]$.

Seguindo o roteiro da Seção 2.2 identificamos os conjuntos $\mathcal{F}_{l o c, \epsilon}^{+}, \mathcal{F}_{b, \epsilon}^{+}, \mathcal{F}_{l o c, 0}^{+}, \mathcal{F}_{b, 0}^{+} \mathrm{e}$ 
as topologias $\Theta_{l o c, \epsilon}^{+}$e $\Theta_{l o c, 0}^{+}$da forma a seguir

$$
\begin{aligned}
\mathcal{F}_{l o c, \epsilon}^{+}=\left\{v: v \in L_{l o c}^{\infty}\left(\mathbb{R}_{+} ; L^{p}(\Omega)\right) \cap L_{l o c}^{\infty}\left(\mathbb{R}_{+} ; H_{d}^{1}(\Omega)\right), \partial_{t} v \in L_{l o c}^{\infty}\left(\mathbb{R}_{+} ; L^{2}(\Omega)\right) \mathrm{e}\right. \\
\left.\partial_{t}^{2} v \in L_{l o c}^{\infty}\left(\mathbb{R}_{+} ; H^{-r}(\Omega)\right)\right\} ; \\
\mathcal{F}_{l o c, 0}^{+}=\left\{v: v \in L_{l o c}^{\infty}\left(\mathbb{R}_{+} ; L^{p}(\Omega)\right) \cap L_{l o c}^{\infty}\left(\mathbb{R}_{+} ; H_{d}^{1}(\Omega)\right) \text { e } \partial_{t} v \in L_{b}^{2}\left(\mathbb{R}_{+} ; L^{2}(\Omega)\right)\right\} ; \\
\mathcal{F}_{b, \epsilon}^{+}=\left\{v: v \in \mathcal{F}_{l o c, \epsilon}^{+} \operatorname{com}\|v\|_{\mathcal{F}_{b, \epsilon}^{+}}<+\infty\right\},
\end{aligned}
$$

onde

$$
\begin{gathered}
\|v\|_{\mathcal{F}_{b, \epsilon}^{+}}=\|v\|_{L^{\infty}\left(\mathbb{R}_{+} ; L^{p}(\Omega)\right)}+\|v\|_{L^{\infty}\left(\mathbb{R}_{+} ; H_{d}^{1}(\Omega)\right)}+\left\|\partial_{t} v\right\|_{L^{\infty}\left(\mathbb{R}_{+} ; L^{2}(\Omega)\right)}+ \\
+\left\|\partial_{t}^{2} v\right\|_{L^{\infty}\left(\mathbb{R}_{+} ; H^{-r}(\Omega)\right)} \\
\mathcal{F}_{b, 0}^{+}=\left\{v: v \in \mathcal{F}_{l o c, 0}^{+} \operatorname{com}\|v\|_{\mathcal{F}_{b, 0}^{+}}<+\infty\right\},
\end{gathered}
$$

onde

$$
\|v\|_{\mathcal{F}_{b, 0}^{+}}=\|v\|_{L^{\infty}\left(\mathbb{R}_{+} ; L^{p}(\Omega)\right)}+\|v\|_{L^{\infty}\left(\mathbb{R}_{+} ; H^{1}(\Omega)\right)}+\left\|\partial_{t} v\right\|_{L_{b}^{2}\left(\mathbb{R}_{+} ; L^{2}(\Omega)\right)}
$$

E munimos $\mathcal{F}_{l o c, \epsilon}^{+}$com a topologia $\Theta_{l o c, \epsilon}^{+}$definida da seguinte forma: $v_{m} \rightarrow v$ em $\Theta_{l o c, \epsilon}^{+}$quando $\Pi_{[0, T]} v_{m} \rightarrow \Pi_{[0, T]} v$ em $\Theta_{T}$ para todo $T>0$. Esta definição é válida para $\epsilon \in\left[0, \epsilon_{0}\right]$. Observemos que a topologia $\Theta_{l o c, 0}^{+}$é mais fraca do que a topologia $\Theta_{l o c, \epsilon}^{+}$.

Sabemos que os espaços de trajetórias $\mathcal{K}_{\epsilon}^{+}(N)$ e $\mathcal{K}_{0}^{+}(N)$ estão contidos em $\mathcal{F}_{b, \epsilon}^{+}$e $\mathcal{F}_{b, 0}^{+}$, respectivamente. Vamos munir esses conjuntos das topologias $\Theta_{l o c, \epsilon}^{+}$e $\Theta_{l o c, 0}^{+}$, respectivamente e denominá-los espaços de trajetórias. O semigrupo translação $\left\{T(t): \mathcal{K}_{\epsilon}^{+}(N) \rightarrow \mathcal{K}_{\epsilon}^{+}(N)\right.$ : $t \geqslant 0\}$ possui um conjunto atrativo para todo $\epsilon>0$, a saber

$$
P=\left\{u \in \mathcal{F}_{b, \epsilon}^{+}: z_{\epsilon}(t) \leqslant 2 \frac{\rho_{\delta}}{\mu_{\delta}}\right\} .
$$

Dos Teoremas 1.3.10 e 4.1 .13 decorre que o semigrupo translação $\{T(t)\}$ definido sobre o espaço de trajetórias $\mathcal{K}_{\epsilon}^{+}(N)$ da equação hiperbólica (4.67) possui um atrator de trajetórias $\mathfrak{A}_{\epsilon}$ para $\epsilon \in\left(0, \epsilon_{0}\right]$. Além disso, o conjunto $\mathfrak{A}_{\epsilon}$ é limitado em $\mathcal{F}_{b, \epsilon}^{+}$, compacto em $\Theta_{l o c, \epsilon}^{+}$e em virtude da estimativa (4.75) também é uniformemente limitado em relação a $\epsilon$ na norma do espaço $\mathcal{F}_{b, 0}^{+}$. 
Vamos denotar por $\mathcal{K}_{\epsilon}$ o núcleo da equação

$$
\epsilon \frac{\partial^{2} u}{\partial t^{2}}+2 \gamma \frac{\partial u}{\partial t}=d \Delta u-f(u)+|u|^{\alpha-1} u \quad(t, x) \in \mathbb{R} \times \Omega
$$

Da definição do espaço $\mathcal{K}_{\epsilon}$, decorre que $\mathcal{K}_{\epsilon} \subset \mathcal{F}_{b, \epsilon}$ e esse espaço consiste das soluções fracas completas de (4.77) satisfazendo as desigualdades

$$
z_{\epsilon}(t) \leqslant \rho_{\delta}
$$

e

$$
2 \gamma \int_{t}^{t+1}\left\|\partial_{t} u\right\|^{2} d t \leqslant \rho_{\delta}+\tilde{c_{1}}|\Omega|
$$

para $t \in \mathbb{R}$.

Analogamente ao Teorema 4.1.13, temos que

$$
\mathfrak{A}_{\epsilon}=\Pi_{+} \mathcal{K}_{\epsilon}, \quad \epsilon \in\left(0, \epsilon_{0}\right],
$$

e o conjunto $\mathcal{K}_{\epsilon}$ é limitado em $\mathcal{F}_{b, \epsilon}$ e compacto em $\Theta_{\text {loc, } \epsilon}$ para $\epsilon>0$.

No caso em que $\epsilon=0$, o semigrupo translação definido sobre o espaço de trajetórias $\mathcal{K}_{0}^{+}$da equação limite parabólica possui um atrator de trajetórias $\mathfrak{A}_{0} \subset \mathcal{F}_{b, 0}^{+}$, compacto em $\Theta_{l o c, 0}$ e $\mathfrak{A}_{0}=\Pi_{+} \mathcal{K}_{0}$, onde $\mathcal{K}_{0}$ é o núcleo da equação

$$
2 \gamma \partial_{t} u=d \Delta u-f(u)+|u|^{\alpha-1} u, \quad t \in \mathbb{R} .
$$

Vamos formular o resultado principal dessa subseção.

Teorema 4.2.8. Se $\mathfrak{A}_{\epsilon}$ é o atrator de trajetórias da equação (4.67) com $\epsilon \in\left(0, \epsilon_{0}\right]$, então a família de atratores $\left\{\mathfrak{A}_{\epsilon}: \epsilon \in\left[0, \epsilon_{0}\right]\right\}$ é semicontínua superiormente em $\epsilon=0$.

Demonstração: Suponha que a tese do enunciado é falsa, logo existem uma vizinhança $\mathcal{O}$ de $\mathcal{K}_{0}$ em $\Theta_{\text {loc }, 0}$ e sequências $\left\{\epsilon_{m}: \epsilon_{m}>0\right\}, \epsilon_{n} \rightarrow 0$ e $\left\{u_{\epsilon_{m}}^{m}\right\} \subset \mathcal{K}_{\epsilon_{m}}$ tais que

$$
u_{\epsilon_{m}}^{m} \notin \mathcal{O}\left(\mathcal{K}_{0}\right)
$$

Da definição do espaço $\mathcal{K}_{0}$, decorre que a sequência $\left\{u_{\epsilon_{m}}^{m}\right\}_{m \in \mathbb{N}}$ é limitada em $\mathcal{F}_{b, 0}$ e existe uma subsequência (vamos manter a notação) tal que $u_{\epsilon_{m}}^{m} \rightarrow \tilde{u}$ em $\Theta_{l o c, 0}$. Além disso, a função $\tilde{u}$ satisfaz as desigualdades

$$
z(t)=J_{\delta}\left(\tilde{u}(t), \partial_{t} \tilde{u}(t)\right) \leqslant \rho_{\delta}
$$


e

$$
2 \gamma \int_{t}^{t+1}\left\|\partial_{t} \tilde{u}\right\|^{2} d t \leqslant \rho_{\delta}+\tilde{c_{1}}|\Omega|
$$

para $t \in \mathbb{R}$.

Vamos demonstrar que $\tilde{u} \in \mathcal{K}_{0}$. Como a função $\tilde{u}$ satisfaz (4.80)-(4.81) é suficiente verificar que $\tilde{u}$ é solução fraca de (4.67) com $\epsilon=0$.

Sabemos que a função $u_{\epsilon_{m}}^{m}$ satisfaz a equação

$$
\epsilon_{m} \partial_{t}^{2} u_{\epsilon_{m}}^{m}+2 \gamma \partial_{t} u_{\epsilon_{m}}^{m}=d \Delta u_{\epsilon_{m}}^{m}-f\left(u_{\epsilon_{m}}^{m}\right)+\left|u_{\epsilon_{m}}^{m}\right|^{\alpha-1} u_{\epsilon_{m}}^{m}
$$

no sentido de distribuição do espaço $D^{\prime}\left(\mathbb{R} ; H^{-r}(\Omega)\right)$.

Para passar o limite em (4.82) devemos verificar que $\epsilon_{m} \partial_{t}^{2} u_{\epsilon_{m}}^{m} \rightarrow 0$ quando $m \rightarrow \infty$, em $D^{\prime}\left(\mathbb{R} ; H^{-r}(\Omega)\right)$. Quanto aos demais termos já sabemos que podemos tomar o limite como foi feito na demonstração do teorema de existência de solução fraca.

De (4.78), resulta que

$$
\epsilon_{m} \int_{\Omega} \frac{\left|\partial_{t} u_{\epsilon_{m}}^{m}\right|^{2}}{2} d x \leqslant \rho_{\delta}, \quad t \in \mathbb{R}
$$

Logo, existe $R>0$ tal que

$$
\int_{-T}^{T} \epsilon_{m}\left\|\partial_{t} u_{\epsilon_{m}}^{m}(s)\right\|_{L^{2}(\Omega)} d s \leqslant\left(\epsilon_{m} 2 T\right)^{\frac{1}{2}}\left(\int_{-T}^{T} \epsilon_{m}\left\|\partial_{t} u_{\epsilon_{m}}^{m}(s)\right\|_{L^{2}(\Omega)}^{2}\right)^{\frac{1}{2}} \leqslant\left(\epsilon_{m} 2 T\right)^{\frac{1}{2}} R,
$$

para todo $T>0$.

Logo, $\epsilon_{m} \partial_{t} u_{\epsilon_{m}}^{m} \rightarrow 0$ quando $m \rightarrow \infty$ em $L^{1}\left(-T, T ; L^{2}(\Omega)\right)$ e como $\partial_{t}$ é um operador contínuo do espaço das distribuições $D^{\prime}\left(-T, T ; L^{2}(\Omega)\right)$ nele próprio, concluímos que $\epsilon_{m} \partial_{t}^{2} u_{\epsilon_{m}}^{m} \rightarrow 0$ em $D^{\prime}\left(-T, T ; L^{2}(\Omega)\right)$ quando $m \rightarrow \infty$ para todo $T>0$. Finalmente, temos que $\epsilon_{m} \partial_{t}^{2} u_{\epsilon_{m}}^{m} \rightarrow 0$ em $D^{\prime}\left(\mathbb{R} ; H^{-r}(\Omega)\right)$ quando $m \rightarrow \infty$.

Consequentemente, $\tilde{u} \in \mathcal{K}_{0}$. Além disso, temos que $u_{\epsilon_{m}}^{m} \rightarrow \tilde{u}$ em $\Theta_{l o c, 0}$, mas isso contradiz a nossa hipótese e com isso terminamos a demonstração.

Usando a imersão $\Theta_{l o c, 0} \subset C\left(\mathbb{R} ; H^{1-\delta}\right)$ para $0<\delta \leqslant 1$, obtemos o seguinte resultado

Corolário 4.2.9. Para todo $T>0$,

$$
\operatorname{dist}_{C\left([0, T] ; H^{1-\delta}\right)}\left(\Pi_{[0, T]} \mathfrak{A}_{\epsilon}, \Pi_{[0, T]} \mathfrak{A}_{0}\right) \rightarrow 0
$$


quando $\epsilon \rightarrow 0^{+}$.

\subsection{Difusão grande}

Nessa seção vamos examinar a questão da continuidade do atrator de trajetórias da equação (4.2) com relação ao coeficiente de difusão $d>0$. Como observamos no início desse capítulo há trabalhos que tratam desse assunto para equações cujo problema de Cauchy correspondente goza da propriedade de unicidade de solução. A nossa investigação vai se restringir ao seguinte problema

$$
\left\{\begin{array}{l}
\frac{\partial^{2} u}{\partial t^{2}}+2 \gamma \frac{\partial u}{\partial t}=d \Delta u-f(u)+|u|^{\alpha-1} u \quad(t, x) \in(0, \infty) \times \Omega \\
\frac{\partial u}{\partial \nu}=0 \quad(t, x) \in(0, \infty) \times \Gamma,
\end{array}\right.
$$

onde $\Omega \subset \mathbb{R}^{n}(n \geqslant 3)$ é um subconjunto aberto, limitado, conexo, com fronteira suave $\Gamma \mathrm{e}$ para cada ponto $x \in \Gamma, \nu=\nu(x)$ indica o vetor normal exterior unitário a $\Gamma$ no ponto $x$. Além disso, $d>0, \alpha \in(0,1)$ e $f: \mathbb{R} \rightarrow \mathbb{R}$ é uma função de classe $C^{1}$ satisfazendo as seguintes hipóteses: existem constantes positivas $\gamma_{0}, \gamma_{1}, \gamma_{2}, c_{1}, c_{2}, C$ e $2<p<\frac{2 n}{n-2}$ tais que

$$
\begin{gathered}
\left|f\left(u_{1}\right)-f\left(u_{2}\right)\right| \leqslant C\left|u_{1}-u_{2}\right|\left(1+\left|u_{1}\right|^{p-2}+\left|u_{2}\right|^{p-2}\right), \\
|f(u)| \leqslant \gamma_{0}\left(|u|^{p-1}+1\right), \\
F(u)=\int_{0}^{u} f(w) d w, \quad F(u) \geqslant \gamma_{1}|u|^{p}-c_{1}, \\
f(u) \cdot u \geqslant \gamma_{2} F(u)-c_{2},
\end{gathered}
$$

para quaisquer $u, u_{1}, u_{2} \in \mathbb{R}$.

Pelo Teorema 4.1 .8 e pela Proposição 4.1.12, para cada $d>0$, a equação (4.83) admite pelo menos uma solução fraca global e possui um atrator de trajetórias $\mathfrak{A}_{d}$.

Sejam $\left\{\lambda_{j}\right\}_{j=0}^{\infty}$ e $\left\{w_{j}\right\}_{j=0}^{\infty}$ os autovalores e as autofunções normalizadas, respectivamente, do problema de Neumann

$$
\left\{\begin{array}{l}
\Delta w+\lambda w=0 \quad \text { em } \Omega \\
\left.\frac{\partial w}{\partial \nu}\right|_{\Gamma}=0
\end{array}\right.
$$

Do Teorema 1.1.29 sabemos que $\lambda_{0}=0<\lambda_{1}<\lambda_{2}<\ldots<\lambda_{j} \rightarrow \infty,\left\{w_{j}\right\}_{j \in \mathbb{N}}$ é um sistema ortonormal completo para $L^{2}(\Omega)$ e $w_{0}(x)=\frac{1}{\sqrt{|\Omega|}}$. 
Consideremos, como fizemos na Seção 3.3, a decomposição ortogonal de $L^{2}(\Omega)=\left[w_{0}\right] \oplus Y$, onde

$$
Y=\left[w_{2}, w_{3}, \ldots\right]=\left\{v: \int_{\Omega} v(x) d x=0\right\}
$$

é o espaço das funções de $L^{2}(\Omega)$ com média nula.

Se $u=u(t, x)$ é uma solução de (3.68), então podemos escrever

$$
u(t, x)=\beta(t) w_{0}(x)+v(x, t)
$$

de modo único, onde

$$
\beta(t)=\int_{\Omega} u(t, x) w_{0}(x) d x=\frac{1}{\sqrt{|\Omega|}} \int_{\Omega} u(t, x) d x
$$

$\mathrm{e}$

$$
v(t, x)=u(t, x)-\beta(t) w_{0}(x) .
$$

A decomposição (4.88) nos permite reescrever a equação (4.83) como um sistema de equações da seguinte forma

$$
\left\{\begin{array}{l}
v_{t t}=-2 \gamma v_{t}+d \Delta v-\tilde{f}\left(\beta w_{0}+v\right)+\frac{1}{|\Omega|} \int_{\Omega} \tilde{f}\left(\beta w_{0}+v\right) d x \\
\beta_{t t}=-2 \gamma \beta_{t}-\frac{1}{\sqrt{|\Omega|}} \int_{\Omega} \tilde{f}\left(\beta w_{0}+v\right) d x
\end{array}\right.
$$

Queremos demonstrar que se $u(t, x)=\beta(t) w_{0}(x)+v(t, x)$ é uma solução da equação (4.83), $d \rightarrow+\infty$ e $\mathfrak{A}_{\infty}$ é o atrator de trajetórias da equação diferencial ordinária

$$
\left\{\begin{array}{l}
\xi^{\prime \prime}(t)+2 \gamma \xi^{\prime}(t)=-f(\xi(t))+|\xi(t)|^{\alpha-1} \xi(t) \\
\xi(0)=\xi_{0} \in \mathbb{R}
\end{array}\right.
$$

então

- na decomposição (4.88), a função componente $v$ satisfaz $v \rightarrow 0$ na topologia $\Theta_{l o c}^{+}$; e

- a família $\left\{\mathfrak{A}_{d}: d>0\right\} \cup\left\{\widetilde{\mathfrak{A}_{\infty}}\right\}$ é semicontínua superiormente em $d=\infty$, onde $\widetilde{\mathfrak{A}_{\infty}}$ é a imersão de $\mathfrak{A}_{\infty}$ em $\left[w_{0}\right] \oplus Y$ através da aplicação $\xi \in \mathbb{R} \mapsto \xi w_{0}$.

Vamos enunciar o nosso resultado principal.

Teorema 4.3.1. Se $\widetilde{\mathfrak{A}_{\infty}}$ é a imersão de $\mathfrak{A}_{\infty}$ em $L^{2}(\Omega)=\left[w_{0}\right] \oplus Y$, então a família $\left\{\mathfrak{A}_{d}\right\}_{d} \cup$ 
$\left\{\widetilde{\mathfrak{A}_{\infty}}\right\}$ é semicontínua superiormente em $d=\infty$, isto é, para toda vizinhança $\mathcal{O}$ de $\widetilde{\mathfrak{A}_{\infty}}$ na topologia $\Theta_{\text {loc }}^{+}$existe $d_{0}>0$ tal que se $d \geqslant d_{0}$, então $\mathfrak{A}_{d} \subset \mathcal{O}\left(\widetilde{\mathfrak{A}_{\infty}}\right)$.

Demonstração: Tomemos $u \in \mathfrak{A}_{d}$. Logo, podemos escrever $u=\beta w_{0}+v$ e $u$ satisfaz

$$
\left\{\begin{array}{l}
v_{t t}=-2 \gamma v_{t}+d \Delta v-\tilde{f}\left(\beta w_{0}+v\right)+\frac{1}{|\Omega|} \int_{\Omega} \tilde{f}\left(\beta w_{0}+v\right) d x \\
\beta_{t t}=-2 \gamma \beta_{t}+G(\beta, 0)+G(\beta, v)-G(\beta, 0)
\end{array}\right.
$$

onde

$$
G(\beta, v)=-\frac{1}{\sqrt{|\Omega|}} \int_{\Omega} \tilde{f}\left(\beta w_{0}+v\right) d x
$$

Lembremos que $\tilde{f}(u)=f(u)-|u|^{\alpha-1} u$.

Vamos demostrar inicialmente que se $d \rightarrow+\infty$, então $v \stackrel{*}{\rightarrow} 0$ em $L^{\infty}\left(0, T ; H^{1}(\Omega)\right)$, $v \stackrel{*}{\rightarrow} 0 \mathrm{em} L^{\infty}\left(0, T ; L^{p}(\Omega)\right), \partial_{t} v \stackrel{*}{\rightarrow} 0 \mathrm{em} L^{\infty}\left(0, T ; L^{2}(\Omega)\right)$ e $\partial_{t}^{2} v \stackrel{*}{\rightarrow} 0 \mathrm{em} L^{\infty}\left(0, T ; H^{-r}(\Omega)\right)$, para todo $T>0$, ou seja, $v \rightarrow 0$ em $\Theta_{l o c}^{+}$.

Pela construção da família da atratores de trajetórias $\left\{\mathfrak{A}_{d}\right\}_{d}$ sabemos que existe um conjunto $B$ limitado na norma de $\mathcal{F}_{b, d}^{+}$(uma bola) tal que $\mathfrak{A}_{d} \subset B$ para todo $d>0$. Observemos que o raio dessa bola independe de $d$ e vamos denotá-lo por $R$.

Tomando o produto da primeira equação de (4.91) por $v_{t}$ e integrando em $\Omega$, obtemos

$$
\frac{1}{2} \frac{d}{d t} \int_{\Omega}\left[\left|v_{t}\right|^{2}+d|\nabla v|^{2}+2 \tilde{F}\left(\beta w_{0}+v\right)\right] d x+2 \gamma \int_{\Omega}\left|v_{t}\right|^{2} d x-\frac{\beta^{\prime}}{\sqrt{|\Omega|}} \int_{\Omega} \tilde{f}\left(\beta w_{0}+v\right) d x=0
$$

Se definirmos

$$
I\left(v, v_{t}\right)=\int_{\Omega}\left[\frac{\left|v_{t}\right|^{2}}{2}+\frac{d|\nabla v|^{2}}{2}+\tilde{F}\left(\beta w_{0}+v\right)\right] d x
$$

então aplicando a Desigualdade de Young (1.3) e usando o fato que existe uma constante positiva $\tilde{L}$ tal que $|\tilde{F}(u)| \leqslant \tilde{L}\left(|u|^{p}+1\right)$, podemos obter constantes positivas $k_{1}, k_{2}, k_{3}$ e $k_{4}$ satisfazendo

$$
\begin{aligned}
& I\left(v, v_{t}\right) \geqslant \min \left\{\frac{1}{2}, \frac{\tilde{\gamma_{1}}}{2^{p+1}}\right\}\left[\left\|v_{t}\right\|_{L^{2}(\Omega)}^{2}+\|v\|_{H^{1}(\Omega)}^{2}+\|v\|_{L^{p}(\Omega)}^{p}\right]-\tilde{c_{1}}|\Omega|-\tilde{\gamma_{1}}\left|\beta w_{0}\right|^{p}|\Omega|- \\
& -\frac{1}{2}\left(\frac{2^{p+1}}{\tilde{\gamma_{1} p}}\right)^{\frac{2}{p-2}}|\Omega| \frac{p-2}{p} \geqslant k_{1}\left[\left\|v_{t}\right\|_{L^{2}(\Omega)}^{2}+\|v\|_{H^{1}(\Omega)}^{2}+\|v\|_{L^{p}(\Omega)}^{p}\right]-k_{2},
\end{aligned}
$$


onde

$$
k_{1}=\min \left\{\frac{1}{2}, \frac{\tilde{\gamma_{1}}}{2^{p+1}}\right\}
$$

$\mathrm{e}$

$$
\begin{gathered}
k_{2}=\tilde{c_{1}}|\Omega|+\tilde{\gamma_{1}} R^{p}+\frac{1}{2}\left(\frac{2^{p+1}}{\tilde{\gamma_{1}} p}\right){ }^{\frac{2}{p-2}}|\Omega| \frac{p-2}{p} ; \\
I\left(v, v_{t}\right) \leqslant \max \left\{\frac{1}{2}, 2^{p} \tilde{L}\right\}\left[\left\|v_{t}\right\|_{L^{2}(\Omega)}^{2}+\|v\|_{H^{1}(\Omega)}^{2}+\|v\|_{L^{p}(\Omega)}^{p}\right]+\tilde{L}|\Omega|+2^{p} \tilde{L}\left|\beta w_{0}\right|^{p}|\Omega| \\
\leqslant k_{3}\left[\left\|v_{t}\right\|_{L^{2}(\Omega)}^{2}+\|v\|_{H^{1}(\Omega)}^{2}+\|v\|_{L^{p}(\Omega)}^{p}\right]+k_{4},
\end{gathered}
$$

onde

$$
k_{3}=\max \left\{\frac{1}{2}, 2^{p} \tilde{L}\right\}
$$

$\mathrm{e}$

$$
k_{4}=\tilde{L}|\Omega|+2^{p} \tilde{L} R^{p} .
$$

Fazendo o produto da primeira equação de (4.91) por $v$, integrando em $\Omega$ e usando a hipótese (4.16), obtemos

$$
\begin{aligned}
& \frac{d}{d t} \int_{\Omega}\left(v \cdot v_{t}+\gamma|v|^{2}\right) d x-\int_{\Omega}\left|v_{t}\right|^{2} d x+d \int_{\Omega}|\nabla v|^{2}+\tilde{\gamma_{2}}|| \beta w_{0}+v \|_{L^{p}(\Omega)}^{p}-\tilde{c_{2}}|\Omega|- \\
& -\frac{\beta}{\sqrt{|\Omega|}} \int_{\Omega} \tilde{f}\left(\beta w_{0}+v\right) d x \leqslant 0 .
\end{aligned}
$$

Somando (4.92) e (4.93) multiplicado por um número positivo $\delta<\min \left\{2 \gamma, 2 k_{1}\right\}$, ficamos com

$$
\begin{aligned}
& \frac{d}{d t} \int_{\Omega}\left[\frac{\left|v_{t}\right|^{2}}{2}+\frac{d|\nabla v|^{2}}{2}+\tilde{F}\left(\beta w_{0}+v\right)+\delta v \cdot v_{t}+\gamma|v|^{2} \delta\right] d x+2 \gamma \int_{\Omega}\left|v_{t}\right|^{2} d x- \\
& -\frac{\beta^{\prime}}{\sqrt{|\Omega|}} \int_{\Omega} \tilde{f}\left(\beta w_{0}+v\right) d x-\delta \int_{\Omega}\left|v_{t}\right|^{2} d x+d \delta \int_{\Omega}|\nabla v|^{2} d x+\tilde{\gamma_{2}} \delta\left\|\beta w_{0}+v\right\|_{L^{p}(\Omega)}^{p}- \\
& -\tilde{c_{2}}|\Omega| \delta-\frac{\delta \beta}{\sqrt{|\Omega|}} \int_{\Omega} \tilde{f}\left(\beta w_{0}+v\right) d x \leqslant 0 .
\end{aligned}
$$

Observemos que podemos assumir a existência do número $\delta$, pois toda solução do espaço de trajetórias satisfaz uma desigualdade como dada em (4.27). 
Se definirmos

$$
\begin{aligned}
J\left(v, v_{t}\right) & =\int_{\Omega}\left[\frac{\left|v_{t}\right|^{2}}{2}+\frac{d|\nabla v|^{2}}{2}+\tilde{F}\left(\beta w_{0}+v\right)+\delta v \cdot v_{t}+\gamma|v|^{2} \delta\right] d x \\
& =I\left(v, v_{t}\right)+\delta \int_{\Omega}\left(v \cdot v_{t}+\gamma|v|^{2}\right) d x
\end{aligned}
$$

então

$$
J\left(v, v_{t}\right) \leqslant\left(k_{3}+\frac{\delta}{2}+\delta \gamma\right)\left[\left\|v_{t}\right\|_{L^{2}(\Omega)}^{2}+\|v\|_{H^{1}(\Omega)}^{2}+\|v\|_{L^{p}(\Omega)}^{p}\right]+k_{4}
$$

e

$$
J\left(v, v_{t}\right) \geqslant\left(k_{1}-\frac{\delta}{2}\right)\left[\left\|v_{t}\right\|_{L^{2}(\Omega)}^{2}+\|v\|_{H^{1}(\Omega)}^{2}+\|v\|_{L^{p}(\Omega)}^{p}\right]-k_{2} .
$$

Em (4.94) vamos aplicar a Desigualdade de Young (1.3), a desigualdade (4.95) e a hipótese (4.14), donde obtemos

$$
\begin{aligned}
& \frac{d}{d t} J\left(v, v_{t}\right) \leqslant-\min \{2 \gamma-\delta, \delta\}\left[\left\|v_{t}\right\|_{L^{2}(\Omega)}^{2}+\|v\|_{H^{1}(\Omega)}^{2}\right]+\tilde{c_{2}}|\Omega| \delta+ \\
& +\left(\frac{\beta^{\prime}+\delta \beta}{\sqrt{|\Omega|}}\right) \int_{\Omega} \tilde{f}\left(\beta w_{0}+v\right) d x-\tilde{\gamma}_{2} \delta\left\|\beta w_{0}+v\right\|_{L^{p}(\Omega)}^{p}+\delta \int_{\Omega}|v|^{2} d x \\
& \leqslant-\min \{2 \gamma-\delta, \delta\}\left[\left\|v_{t}\right\|_{L^{2}(\Omega)}^{2}+\|v\|_{H^{1}(\Omega)}^{2}\right]+\tilde{c_{2}}|\Omega| \delta+\frac{\tilde{\gamma_{2}} \delta}{2}\left\|\beta w_{0}+v\right\|_{L^{p}(\Omega)}^{p}+ \\
& +\tilde{\gamma_{0}} \tilde{R}\left(\frac{2 \tilde{\gamma_{0}} \tilde{R}(p-1)}{\tilde{\gamma_{2}} \delta p}\right)^{p-1}|\Omega| \frac{1}{p}+\tilde{\gamma_{0}} R|\Omega|-\tilde{\gamma}_{2} \delta\left\|\beta w_{0}+v\right\|_{L^{p}(\Omega)}^{p}+\delta \int_{\Omega}|v|^{2} d x \\
& \leqslant-\min \left\{2 \gamma-\delta, \delta, \frac{\tilde{\gamma_{2}} \delta}{2^{p+2}}\right\}\left[\left\|v_{t}\right\|_{L^{2}(\Omega)}^{2}+\|u\|_{H^{1}(\Omega)}^{2}+\|v\|_{L^{p}(\Omega)}^{p}\right]+\tilde{c_{2}}|\Omega| \delta+ \\
& +\tilde{\gamma_{0}} \tilde{R}\left(\frac{2 \tilde{\gamma_{0}} \tilde{R}(p-1)}{\tilde{\gamma_{2}} \delta p}\right)^{p-1}|\Omega| \frac{1}{p}+\tilde{\gamma_{0}} \tilde{R}|\Omega|+\delta\left(\frac{2^{p+3}}{\tilde{\gamma_{2}} p}\right)^{\frac{2}{p-2}}|\Omega| \frac{p-2}{p}+\frac{\tilde{\gamma}_{2} R^{p} \delta}{2} \\
& \leqslant-k_{5} J\left(v, v_{t}\right)+k_{6},
\end{aligned}
$$

onde usamos a limitação

$$
\left|\frac{\beta^{\prime}+\delta \beta}{\sqrt{|\Omega|}}\right| \leqslant \tilde{R} .
$$

Observemos que em (4.97) as constantes positivas $k_{5}$ e $k_{6}$ são independentes de $d$ e 
são dadas por

$$
k_{5}=\frac{\min \left\{2 \gamma-\delta, \delta, \frac{\tilde{\gamma_{2}} \delta}{2^{p+2}}\right\}}{\left(k_{3}+\frac{\delta}{2}+\delta \gamma\right)}
$$

e

$$
\begin{aligned}
k_{6} & =k_{4} k_{5}+\tilde{c_{2}}|\Omega| \delta+\tilde{\gamma_{0}} \tilde{R}\left(\frac{2 \tilde{\gamma_{0}} \tilde{R}(p-1)}{\tilde{\gamma_{2}} \delta p}\right)^{p-1}|\Omega| \frac{1}{p}+ \\
& +\tilde{\gamma_{0}} \tilde{R}|\Omega|+\delta\left(\frac{2^{p+3}}{\tilde{\gamma_{2} p}}\right)^{\frac{2}{p-2}}|\Omega| \frac{p-2}{p}+\frac{\tilde{\gamma_{2}} R^{p} \delta}{2} .
\end{aligned}
$$

Portanto,

$$
J\left(v, v_{t}\right) \leqslant e^{-k_{5} t} J\left(v_{0}, \partial_{t} v_{0}\right)+\frac{k_{6}}{k_{5}} .
$$

De (4.96) e (4.98), temos que

$$
\|\nabla v\|_{L^{2}(\Omega)}^{2} \leqslant \frac{1}{d}\left[\left(k_{1}-\frac{\delta}{2}\right)^{-1}\left(e^{-k_{5} t} J\left(v_{0}, \partial_{t} v_{0}\right)+\frac{k_{6}}{k_{5}}\right)+k_{2}\left(k_{1}-\frac{\delta}{2}\right)^{-1}\right] \rightarrow 0
$$

quando $d \rightarrow+\infty$. De (4.99), segue que

$$
\|\nabla v\|_{L^{\infty}\left(0, T ; L^{2}(\Omega)\right)} \rightarrow 0
$$

quando $d \rightarrow+\infty$ e, em particular, $\nabla v \stackrel{*}{\rightarrow} 0$ em $L^{\infty}\left(0, T ; L^{2}(\Omega)\right)$ quando $d \rightarrow+\infty$.

Pela desigualdade de Poincaré e de (4.100), decorre que

$$
\|v\|_{L^{\infty}\left(0, T ; L^{2}(\Omega)\right)} \rightarrow 0
$$

quando $d \rightarrow+\infty$. Logo, $v \stackrel{*}{\rightarrow} 0$ em $L^{\infty}\left(0, T ; L^{2}(\Omega)\right)$ quando $d \rightarrow+\infty$.

Portanto,

$$
\|v\|_{L^{\infty}\left(0, T ; H^{1}(\Omega)\right)} \rightarrow 0
$$

quando $d \rightarrow+\infty$ e $v \stackrel{*}{\rightarrow} 0$ em $L^{\infty}\left(0, T ; H^{1}(\Omega)\right)$ quando $d \rightarrow+\infty$.

Agora, pela desigualdade de Gagliardo-Nirenberg existe uma constante positiva $K$ 
tal que

$$
\|v(t)\|_{L^{p}(\Omega)}^{p} d t \leqslant K^{p}\|v(t)\|_{L^{2}(\Omega)}^{(1-\theta) p}\|v(t)\|_{H^{1}(\Omega)}^{\theta p} \leqslant K^{p}\|v\|_{L^{\infty}\left(0, T ; L^{2}(\Omega)\right)}^{(1-\theta) p}\|v\|_{L^{\infty}\left(0, T ; H^{1}(\Omega)\right)}^{\theta p} \rightarrow 0,
$$

quando $d \rightarrow+\infty$. Assim, $v \stackrel{*}{\rightarrow} 0$ em $L^{\infty}\left(0, T ; L^{p}(\Omega)\right)$ quando $d \rightarrow+\infty$.

Da continuidade da aplicação derivada $\partial_{t}: \mathcal{D}^{\prime}\left(0, T ; L^{2}(\Omega)\right) \rightarrow \mathcal{D}^{\prime}\left(0, T ; L^{2}(\Omega)\right)$ e como $L^{\infty}\left(0, T ; L^{2}(\Omega)\right) \hookrightarrow \mathcal{D}^{\prime}\left(0, T ; L^{2}(\Omega)\right)$ e $v \rightarrow 0$ em $L^{\infty}\left(0, T ; L^{2}(\Omega)\right)$ quando $d \rightarrow+\infty$, segue que

$$
\partial_{t} v \stackrel{*}{\rightarrow} 0 \quad \text { em } \quad L^{\infty}\left(0, T ; L^{2}(\Omega)\right)
$$

quando $d \rightarrow+\infty$. Usando novamente esse argumento de continuidade da aplicação derivada, decorre que

$$
\partial_{t}^{2} v \stackrel{*}{\rightarrow} 0 \quad \text { em } \quad L^{\infty}\left(0, T ; H^{-r}(\Omega)\right)
$$

quando $d \rightarrow+\infty$.

Concluímos que $v \rightarrow 0$ em $\Theta_{l o c}^{+}$quando $d \rightarrow+\infty$.

Como a equação diferencial ordinária

$$
\xi_{t t}+2 \gamma \xi_{t}=-f(\xi)+|\xi|^{\alpha-1} \xi=-\tilde{f}(\xi)
$$

possui um atrator de trajetórias $\mathfrak{A}_{\infty}$, podemos considerá-lo imerso no espaço de trajetórias $K^{+}$da equação hiperbólica por meio da aplicação $\xi \mapsto \xi w_{0}$, sendo denotado por $\widetilde{\mathfrak{A}_{\infty}}$.

Da mesma forma que demonstramos no Teorema (3.3.2), segue que $|G(\beta, v)-G(\beta, 0)| \rightarrow$ 0 quando $v \rightarrow 0$ em $\Theta_{l o c}^{+}$, ou seja, $G$ é contínua com respeito a $v$.

Portanto, do que fizemos antes, para toda vizinhança de $\widetilde{\mathfrak{A}_{\infty}}$ na topologia $\Theta_{l o c}^{+}$existe $d_{0}>0$ tal que para $d \geqslant d_{0}$ temos $\mathfrak{A}_{d} \subset \mathcal{O}\left(\widetilde{\mathfrak{A}_{\infty}}\right)$. 


\section{Bibliografia}

[1] Adams, R. and Fournier, J. Sobolev spaces, Pure and applied mathematics series, Second Edition, Academic Press, 2003.

[2] Babin A. V. and Vishik, M. I. Attractors of evolutions equations, Nauka, Moscow, 1989; English trans., North-Holland, Amsterdam, 1992.

[3] Besov, O. V. , Il'in, V. P. and Nikol'skii, S. M. Integral representations of functions and embedding theorems, Nauka, Moscow, 1975; English transl., Washington, DC, 1979.

[4] Brézis, H. Análisis funcional: Teoria y aplicaciones, Alianza Editorial, 1984.

[5] Carvalho, A. N. Infinite dimensional dynamics described by ordinary differential equations, J. Differential Equations, 116, 338-404, 1995.

[6] Carvalho, A. N. Large diffusion with dispersion, Nonlinear Analysis - Theory, Methods e Applications, 17, 1139-1151, 1991.

[7] Carvalho, A. N. Spatial homogeneity in damped hyperbolic equations, Dynamic Systems and Applications, 1, 221-250, 1992.

[8] Cavalcanti, M. M. and Domingos Cavalcanti, V. N. Introdução à teoria das distribuições e aos espaços de Sobolev, Maringá: Textos do Depto. de Matemática - UEM, 2007.

[9] Chepyzhov, V. V. and Vishik, M. I. Attractors for equations of mathematical physics, Colloquium publications, 49, American Mathematical Society, Providence, RI, 2002.

[10] Chepyzhov, V. V. and Vishik, M. I. Trajectory attractors of equations of mathematical physics, Uspekhi Mat. Nauk, 66, 3-102, 2011.

[11] Chepyzhov, V. V. and Vishik, M. I. Trajectory attractors for evolution equations, C. R. Acad. Sci. Paris Sér. I Math., 321, 1309-1314, 1995.

[12] Chepyzhov, V. V. and Vishik, M. I. Evolutions equations and their trajectory attractors, J. Math. Pures Appl., 76, 913-964, 1997. 
[13] Chepyzhov, V. V. and Vishik, M. I. Trajectory attractors for reaction-diffusion systems, Topol. Methods Nonlinear Anal., 7, 49-76, 1996.

[14] Chepyzhov, V. V., Vishik, M. I. and Zelik, S. V. Strong trajectory attractor for a dissipative reaction-diffusion system, Doklady Mathematics, 82, 869-873, 2010.

[15] Chepyzhov, V. V. and Vishik, M. I. Trajectory attractors for reaction-diffusion systems with a small diffusion coefficient, Doklady Mathematics, 79, 227-230, 2009.

[16] Chepyzhov, V. V. and Vishik, M. I. Trajectory attractors of reaction-diffusion systems with small difusion, Sbornik: Mathematics, 200, 471-497, 2009.

[17] Chepyzhov, V. V. and Vishik, M. I. Global attractor and its perturbations for a dissipative hyperbolic equation, Russian Journal of Mathematical Physics, 8, 311-330, 2001.

[18] Coddington,E. A. and Levinson, N. Theory of ordinary differential equations, McGrawHill, New York, 1955.

[19] Conway, E., Hoff, D. and Smoller, J. Large time behavior of solutions of systems of nonlinear reaction-diffusion equations, SIAM J. Appl. Math, 35, 1-16, 1978.

[20] Dunford, N and Schwartz, J. T. Linear operators, Wiley-Interscience, New York, 1971.

[21] Engelkin, R. General Topology, Revised and completed edition, Berlin: Heldermann, 1989.

[22] Hale, J. K. Large diffusivity and asymptotic behavior in parabolic systems, J. Math. Anal. Appl., 118, 455-466, 1986.

[23] Hale, J. Asymptotic behavior and dynamics in infinite dimensions, in Nonlinear Differential Equations, J. Hale and P. Martinez-Amores, eds., Pitman, Boston, 1985.

[24] Hale, J. K. Hale and Rocha, C. Varying boundary conditions with large diffusivity, J. Math. Pures Appl., 66, 139-158, 1987.

[25] Hale, J. K. Hale and Rocha, C. Interaction of diffusion and boundary conditions, Nonlinear Analysis - Theory, Methods and Applications, 11, 633-649, 1987.

[26] Jost, J. Partial Differential Equations, Graduate Texts in Mathematics 214, SpringerVerlag, Second Edition, 2007.

[27] Kapustyan, O. V. and Valero, J. Comparison between trajectory and global attractors for evolution systems without uniqueness of solutions, International Journal of Bifurcation and Chaos, 20, 2723-2734, 2010.

[28] Kolmogorov, A. N. and Fomin, S. V. Elements of the theory of functions and functional analysis, Nauka, Moscow, 1981. 
[29] Lions, J. L. Quelques méthodes de résolution des problèmes aux limites non linéaires, Dunod, Paris, 1969.

[30] Lions, J. L and Magenes, E. Problèmes aux limites non homogènes et applications, 1, Gauthier-Villars, Paris, 1968.

[31] Lyapin, A. S. On the limit behavior of the trajectory attractor of a nonlinear hyperbolic equation containing a small parameter at the highest derivate, Nauka, Moscow, 1981.

[32] Matos, M. Integral de Bochner e espaços $L^{p}(0, t ; X)$, apostila do prof. Marivaldo P. Matos.

[33] Medeiros, L. A. Lições de equações diferenciais parciais, Rio de Janeiro: UFRJ, Instituto de Matemática, 2002.

[34] Medeiros, L. A. and Milla Miranda, M. Introdução aos Espaço de Sobolev e as Equações Direnciais Parciais, Instituto de Matemática, UFRJ, Rio de Janeiro, RJ, 1986.

[35] Medeiros, L. A. and Milla Miranda, M. Espaços de Sobolev(Iniciação aos Problemas Elíticos não Homogêneos, Instituto de Matemática, UFRJ, Rio de Janeiro, RJ, 1999.

[36] Milla Miranda, M. Trace for the dual of Sobolev Spaces, Bol. Soc. Paranaense de Matemática, 11, 131-157, 1992.

[37] Miranville, A. and Zelik, S. Attractors for dissipative partial differential equations in bounded and unbounded domains, in Handbook of Differential Equations, Evolutionary Partial Differential Equations, C. M. Dafermos and M. Pokorny eds., Elsevier, Amsterdam.

[38] Munkres, J. R. Topology: a first course, Prentice-Hall, Inc., Englewood Cliffs, New Jersey, 1975.

[39] Pereira, A. L. and de Oliveira, L. A. F. Invariant manifolds and limiting equations for a hyperbolic problem, Dynamics of Continuous, Discrete and Impulsive Systems, 7, 503524,2000 .

[40] Robinson, J. C. Infinite dimensional dynamical systems: An introduction to dissipative parabolic PDEs and the theory of global attractors, Cambridge University Press, 2001.

[41] Schaefer, H. H. Topological vector spaces, Graduate texts in mathematics 3, New York: Springer, c1999.

[42] Sell, G. R. and You, Y. Dynamics of evolutionary equations, Springer, New York, 2002.

[43] Smoller, J. Shock waves and reaction-diffusion equations, Springer-Verlag, New York Inc, 1983. 
[44] Sola-Morales,J. and Valencia, M. Trend to spatial homogeneity for solutions to semilinear damped wave equations, Proceedings of the Royal Society of Edinburgh, 105A, 117-126, 1987.

[45] Sotomayor, J. Lições de equações diferenciais ordinárias, Rio de Janeiro: IMPA, Projeto Euclides, 1979.

[46] Temam, R. Infinite dimensional dynamical systems in mechanics and physics, SpringerVerlag, New York, 1997.

[47] Temam, R. On the theory and numerical analysis of the Navier-Stokes equations, Amer. Math. Soc., Providence, RI, 2001.

[48] Temam, R. Navier-Stokes equations: theory and numerical analysis, AMS Chelsea Publishing, 2001.

[49] Triebel, H. Interpolation theory, functional spaces, differential operators, North-Holland, Amsterdam-NewYork, 1978.

[50] Yosida, K. Functional Analysis, Sixth Edition, Springer-Verlag, Berlin Heidelberg New York 1980.

[51] Yue, G.; Zhong, C. Limiting behavior of trajectory attractor of perturbed reactiondiffusion equations (preprint). 